\title{
Passive Macromodeling of High-Speed Circuits with Embedded Time-Delays
}

by

\author{
Andrew Charest
}

A thesis submitted to the Faculty of Graduate and Postdoctoral Affairs

in partial fulfilment of the requirements for the degree of

Doctor of Philosophy

in

Electrical and Computer Engineering

Ottawa-Carleton Institute for Electrical and Computer Engineering Carleton University

Ottawa, Canada

(C) 2012

Andrew Charest 
Library and Archives

Canada

Published Heritage

Branch

395 Wellington Street

Ottawa ON K1A ON4

Canada
Bibliothèque et

Archives Canada

Direction du

Patrimoine de l'édition

395 , rue Wellington

Ottawa ON K1A ON4

Canada
Your file Votre référence

ISBN: 978-0-494-89326-5

Our file Notre référence

ISBN: 978-0-494-89326-5

\section{NOTICE:}

The author has granted a nonexclusive license allowing Library and Archives Canada to reproduce, publish, archive, preserve, conserve, communicate to the public by telecommunication or on the Internet, loan, distrbute and sell theses worldwide, for commercial or noncommercial purposes, in microform, paper, electronic and/or any other formats.

The author retains copyright ownership and moral rights in this thesis. Neither the thesis nor substantial extracts from it may be printed or otherwise reproduced without the author's permission.
AVIS:

L'auteur a accordé une licence non exclusive permettant à la Bibliothèque et Archives Canada de reproduire, publier, archiver, sauvegarder, conserver, transmettre au public par télécommunication ou par l'Internet, prêter, distribuer et vendre des thèses partout dans le monde, à des fins commerciales ou autres, sur support microforme, papier, électronique et/ou autres formats.

L'auteur conserve la propriété du droit d'auteur et des droits moraux qui protege cette thèse. $\mathrm{Ni}$ la thèse ni des extraits substantiels de celle-ci ne doivent être imprimés ou autrement reproduits sans son autorisation.
In compliance with the Canadian Privacy Act some supporting forms may have been removed from this thesis.

While these forms may be included in the document page count, their removal does not represent any loss of content from the thesis.
Conformément à la loi canadienne sur la protection de la vie privée, quelques formulaires secondaires ont été enlevés de cette thèse.

Bien que ces formulaires aient inclus dans la pagination, il n'y aura aucun contenu manquant. 


\section{Abstract}

As designers integrate an ever-increasing diversity of on-board optical components, analog circuits, digital blocks, and Micro-Electro-Mechanical (MEM) devices in each product, ensuring the signal integrity of modern electronic devices has become a challenging task. Coupled with higher operating speeds, sharper excitations, and denser circuit layouts, signal degradation from effects such as reflection, crosstalk, delays, attenuation and Electro-Magnetic Interference (EMI) can no longer be neglected in the modeling and simulation stage of the design cycle. For many components, it is not always possible to find analytical models that accurately capture all of these effects. In such cases, the behavior of these modules is generally characterized by sampled/tabulated data (in the admittance, impedance, scattering, or hybrid domains), obtained either directly from measurements or from a 3D Electro-Magnetic (EM) field solver.

Including tabulated data of electrically long networks in SPICE simulators has been a topic of intense research in the past few years. Recently, novel compact macromodeling algorithms for electrically long tabulated data networks were proposed based on approximating the data using Delayed Rational Functions (DRFs). However, DRFbased techniques do not guarantee the passivity of the resulting model, which is necessary for performing stable and accurate transient analysis. In addition, conventional DRF models approximate each delayed response using a separate delayed rational term. For tabulated data networks with multiple delayed responses corresponding to 
periodic reflections, a large number of delayed rational terms may be needed resulting in a high-order model and slow transient analysis. Also, due to the increasing number and diversity of devices in modern designs, global transient analysis of large systems of delay differential network equations has become a computationally expensive task. Recently, model-order reduction algorithms were introduced for efficient unified simulation of these large time-delay systems. However, these algorithms do not guarantee the passivity of the resulting reduced-order macromodel.

In this thesis, efficient passivity verification and compensation techniques are developed for macromodels based on DRFs obtained from tabulated scattering or admittance parameter data. For passivity verification, the necessary search region is reduced from the entire right-half of the complex plane to a single finite interval along the imaginary axis. For passivity compensation, the residues of the DRFs are iteratively perturbed until the model becomes passive. In addition, an innovative macromodeling technique is presented for tabulated data networks with multiple delays that correspond to periodic reflections. The proposed algorithm is based on a novel DRF formulation that captures multiple delays using only a single delayed rational term. Furthermore, a nèw passive model-order reduction algorithm is introduced for efficient unified transient analysis of large circuits with embedded delay-based macromodels. The proposed algorithms enable an efficient analysis of long delay interconnects in SPICE-like analog circuit simulators. 
To my family 


\section{Acknowledgments}

I owe my deepest gratitude to my supervisors Professor Michel Nakhla and Professor Ramachandra Achar, without their expert guidance and wisdom this thesis would not have been possible. Their always encouraging and friendly manner made my graduate studies an enjoyable and enriching experience I will never forget. I am honored to have worked with these two truly inspirational scholars.

I am indebted to Professor Tom Smy for giving me summer research positions throughout my undergraduate years. With boundless patience and understanding he taught me many of the founding principles necessary for this work. I would also like to thank Professor Pavan Gunupudi for his lively and insightful discussions over the years. Many thanks are also extended to Dr. Changzhong Chen, Dr. Dharmendra Saraswat, Dr. Ihsan Erdin, and Dr. Nick Soveiko. Their helpful comments and feedback contributed essential expertise and technical advice that greatly improved the quality of this work.

Numerous organizations have also helped contribute to the quality of this research. I would like to thank Tyco Electronics Inc., Celestica Inc., and CST GmbH for providing examples of practical engineering problems and applications. Furthermore, I am very grateful to the Natural Science and Engineering Research Council of Canada (NSERC) and the Ontario Ministry of Training, Colleges, and Universities for providing funding and financial support throughout my graduate studies. This enabled 
me to publish peer-reviewed papers, attend international conferences and gain direct academic and industry experience. Overall, this greatly improved the caliber of my education.

I would also like to acknowledge and thank all my friends and colleagues for making my education such a memorable experience. Working in the Computer-Aided Design group at Carleton University, I feel very fortunate to have had the opportunity to meet and connect with so many wonderful people.

Last but not least, I give special thanks to my family for all their unconditional love, encouragement, and support. To my loving wife, Diana, I profess my profound appreciation for her eternally warm demeanor and heavenly cooking that kept me uplifted, enthusiastic, and gave me the strength to keep going. Most of all, I am forever grateful to my parents. Words cannot express my admiration for the endless affection, dedication, and sacrifice they have provided for their children. 


\section{Table of Contents}

Abstract $\ldots \ldots \ldots \ldots \ldots \ldots \ldots \ldots \ldots \ldots \ldots \ldots \ldots \ldots \ldots \ldots \ldots$

Acknowledgements ................... vi

Table of Contents $\ldots \ldots \ldots \ldots \ldots \ldots \ldots \ldots \ldots \ldots \ldots \ldots \ldots$

List of Tables $\ldots \ldots \ldots \ldots \ldots \ldots \ldots \ldots \ldots \ldots \ldots \ldots \ldots \ldots \ldots$

List of Figures $\ldots \ldots \ldots \ldots \ldots \ldots \ldots \ldots \ldots \ldots \ldots \ldots$ xvii

List of Abbreviations $\ldots \ldots \ldots \ldots \ldots \ldots \ldots$ xviii

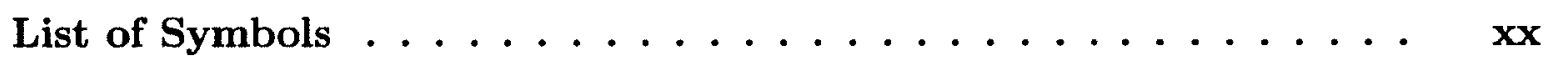

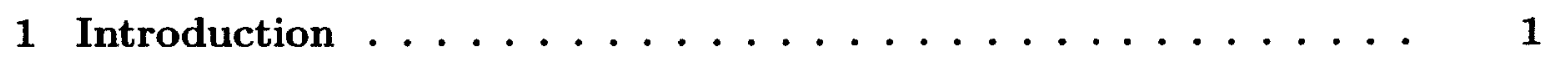

1.1 Background and Motivation $\ldots \ldots \ldots \ldots \ldots \ldots$

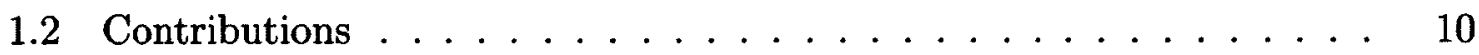

1.3 Organization of the Thesis $\ldots \ldots \ldots \ldots \ldots \ldots \ldots$

2 Review of Modeling Techniques for Tabulated Data Networks • 13

2.1 Review of Simulation and Modeling for Tabulated Data Networks . . 14

2.2 Rational Fitting Techniques $\ldots \ldots \ldots \ldots \ldots \ldots$

2.2.1 The Vector Fitting Algorithm . . . . . . . . . . . 17 
2.3 Macromodeling Issues: Stability, Causality, and Passivity . . . . . . 26

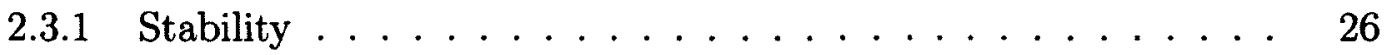

2.3 .2 Causality ......................... 27

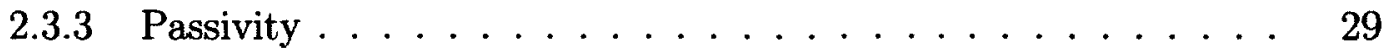

2.4 Passive Rational Function based Macromodeling . . . . . . . . . . . . 32

2.4 .1 Passivity Verification . . . . . . . . . . . . 33

2.4.2 Passivity Compensation .............. 37

3 Review of Macromodeling Networks with Time-Delays . . . . 44

3.1 Long Delay Networks: Macromodeling Issues . . . . . . . . . . . . 45

3.1.1 High-Order Rational Approximations . . . . . . . . . . 45

3.1.2 Ringing in the Response . . . . . . . . . . . . . 46

3.1.3 Inaccurate Timing Analysis . . . . . . . . . . . . . . . 49

3.2 Macromodeling Techniques for Time-Delay Networks . . . . . . . . 52

3.3 Delay-based Macromodeling via Hilbert Transforms . . . . . . . . . 54

3.4 Delay-based Macromodeling via Gabor Transforms . . . . . . . . . 56

3.4.1 Delay Estimation using Time-Frequency Analysis . . . . . . 57

3.4.2 Construction of Delayed Rational Functions (DRFs) . . . . . 58

3.5 The Delayed Vector Fitting Algorithm . . . . . . . . . . . 60

3.5.1 Stage 1: Pole Identification .............. 61

3.5.2 Stage 2: Residue Identification . . . . . . . . . . . 64

4 Proposed Passive Macromodeling Algorithm for Scattering Parameter Based Delayed Rational Functions . . . . . . . . 65

4.1 Introduction . . . . . . . . . . . . . . . . 66

4.2 Problem Formulation . . . . . . . . . . . . . . . 67 
4.3 Proposed Passivity Verification Algorithm . . . . . . . . . . . 70

4.3.1 Reduction of the Search Region to the Imaginary Axis . . . . 71

4.3.2 Reduction of the Search Region to a Single Finite Period on the Imaginary Axis . . . . . . . . . . . 75

4.4 Development of a Half-Size FDGEP for Passivity Verification . . . . . 79

4.5 Identification of Passivity Verification Regions . . . . . . . . . . 88

4.6 Proposed Passivity Compensation Algorithm . . . . . . . . . . . . . . 89

4.7 Numerical Results . . . . . . . . . . . . . . . . . . 92

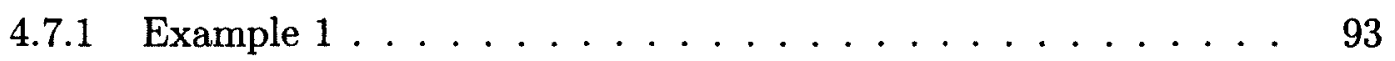

4.7 .2 Example 2....................... 96

4.7.3 Example $3 \ldots \ldots . \ldots \ldots 9$

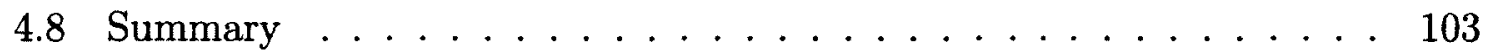

5 Passive Macromodeling Algorithm for Admittance Parameter

Based Delayed Rational Functions . . . . . . . . . . . . 104

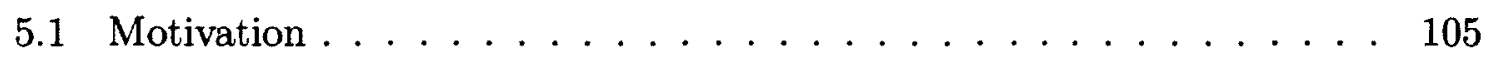

5.2 Problem Formulation . . . . . . . . . . . . . . . 110

5.3 Proposed Passivity Verification Algorithm . . . . . . . . . . . 113

5.3.1 Reduction of the Search Region to the Imaginary Axis . . . . 114

5.3.2 Reduction to a Finite Search Region . . . . . . . . . . . . 117

5.3.3 Identification of Passivity Violation Regions . . . . . . . . . . 120

5.4 Proposed Passivity Compensation Algorithm . . . . . . . . . . . . . . 121

5.4.1 Perturbation of Residues . . . . . . . . . . . . . . . 121

5.4 .2 Error Control . . . . . . . . . . . . . 125

5.5 Numerical Results . . . . . . . . . . . . . . . . . 126

5.5.1 Example 1................... 127 
$5.5 .2 \quad$ Example $2 \ldots \ldots \ldots \ldots \ldots \ldots \ldots \ldots \ldots \ldots$

5.5 .3 Example $3 \ldots \ldots \ldots \ldots \ldots \ldots \ldots$

5.6 Summary . . . . . . . . . . . . . . . . . . . . . 140

6 Stable Periodic Delayed Rational Function Based Macromodels for Tabulated Data Networks . . . . . . . . . . . . 141

6.1 Introduction . . . . . . . . . . . . . . . . . . 142

6.2 Problem Formulation and Review . . . . . . . . . . . . 143

6.3 Construction of Periodic Delayed Rational Functions . . . . . . . 145

6.4 Verification and Enforcement of Stability . . . . . . . . . . . 147

6.5 Numerical Results . . . . . . . . . . . . . . . . . . . . . 148

6.5 .1 Example $1 \ldots \ldots \ldots \ldots \ldots \ldots \ldots \ldots$

6.5 .2 Example $2 \ldots \ldots \ldots \ldots \ldots \ldots \ldots$

6.6 Summary . . . . . . . . . . . . . . . . . . 157

7 Passive Model-Order Reduction of RLC Circuits with Embedded Time-Delay Descriptor (TDD) Systems . . . . . . . . . . 158

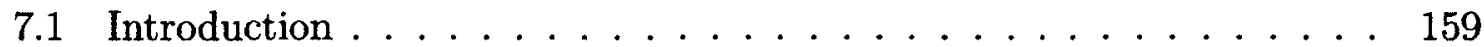

7.2 Problem Formulation . . . . . . . . . . . . . . . . . . 161

7.2.1 Formulation of Network Equations _. . . . . . . . . 161

7.2.2 Model-Order Reduction of Time-Delay Systems . . . . . . . 163

7.2.3 Passivity Criteria for Time-Delay Systems . . . . . . . . . . 164

7.3 Proposed Passive Reduction Algorithm . . . . . . . . . . . . . 165

7.3.1 Passivity Verification of TDD Systems _... . . . . . 166

7.3.2 Formulation of Unified Network Equations . . . . . . . . . 169

7.3.3 Passive Model-Order Reduction . . . . . . . . . . . . 170 
7.3.4 Proof of Preservation of Passivity . . . . . . . . . 171

7.4 Numerical Results . . . . . . . . . . . . . . . . . . . . 174

7.4.1 Example 1..................... 174

7.4 .2 Example 2 . . . . . . . . . . . . 177

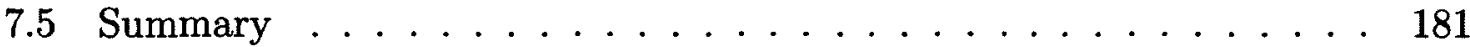

8 Conclusions and Future Research . . . . . . . . . . 182

8.1 Summary . . . . . . . . . . . . . . . . 182

8.2 Future Research . . . . . . . . . . . . . . . . . 184

Bibliography .......................... 201 


\section{List of Tables}

4.1 A comparison of eigenvalues from the full-size and half-size FDGEP at

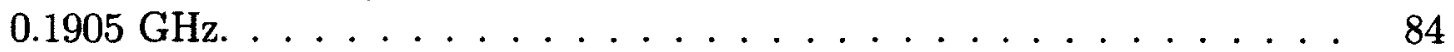

4.2 A comparison of CPU time for obtaining an upper bound from the full-size and half-size FDGEP. . . . . . . . . . . . . . . .

6.1 A comparison of macromodel accuracy for example 1 (DRF: Delayed Rational Function; RMSE: Root Mean Squared Error). . . . . . . . . . . 151

6.2 A comparison of macromodel accuracy for example 2 (DRF: Delayed Rational Function; RMSE: Root Mean Squared Error). . . . . . . . . . . . 154 


\section{List of Figures}

1.1 Illustration of signal integrity issues (PCB: Printed Circuit Board; IC: Interconnect Circuit) . . . . . . . . . . . . . .

1.2 Illustration of sample time and frequency-domain responses for a long delay line. . . . . . . . . . . . . . . . 5

1.3 Illustration of periodic delays in the transient response and the corresponding requirements for a DRF approximation.

2.1 Unstable transient response monotonically and exponentially increasing in magnitude.

2.2 Unstable transient response with spurious oscillations. . . . . . . . . 28

2.3 Illustration of a stable, but nonpassive macromodel connected with passive terminations. The overall network is unstable. . . . . . . . . . 29

2.4 Illustration of iterative perturbation process. . . . . . . . . . . 38

3.1 Two-port lossless transmission line network. . . . . . . . . . . . 46

3.2 Illustration of port-to-port delay from a lossless transmission line. . . . . 47

3.3 Illustration of spurious ringing effect from approximating an exponential delay function using a rational function. . . . . . . . . 50

3.4 Illustration of spurious ringing effect after rising clock edge. $\ldots \ldots \ldots 51$

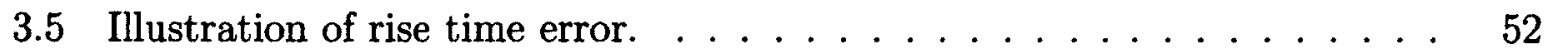

4.1 Semicircular arc in the right-half of the complex plane. . . . . . . . . 74

4.2 Eigenvalue spectrum of the full-size FDGEP for example $1 \ldots \ldots$. . . . . 93 
4.3 Passivity violations of the DRFs for example $1 . \ldots . \ldots 9$

4.4 Accuracy comparison of the scattering parameters for example 1 (the Original Tabulated Data, Nonpassive Macromodel, and Passive Macromodel are all overlapping). . . . . . . . . . . . . . . . 97

4.5 Eigenvalue spectrum of the full-size FDGEP for example 2. . . . . . . . . 98

4.6 Passivity violations of the DRFs for example $2 \ldots \ldots$. . . . . . . . 99

4.7 Comparison of scattering parameters for example 2 (the Original Tabulated Data, Nonpassive Macromodel, and Passive Macromodel are all overlapping). . . . . . . . . . . . . . . . .

4.8 Electrical system for example $3 . \ldots \ldots$. . . . . . . . . . . . . 100

4.9 Differential trace geometry for example $3 . \ldots \ldots$. . . . . . . . . . 100

4.10 Scattering parameters for example 3. . . . . . . . . . . . . . . 101

4.11 Eigenvalue spectrum of the full-size FDGEP for example 3. . . . . . . . . 102

4.12 Passivity verification of the DRFs for example $3 . \ldots \ldots 2$

5.1 Comparison of the norm of the scattering parameters in the frequencydomain. ...................... 107

5.2 Comparison of the real part of the scattering parameters in the frequencydomain. . . . . . . . . . . . . . . . 108

5.3 Comparison of the real part of the admittance parameters in the frequencydomain. . . . . . . . . . . . . . . . .

5.4 Circuit illustrating the effect of a small perturbation in the scattering domain model. . . . . . . . . . . . . . . . . . .

5.5 Time-domain response showing the effect of a small perturbation in scattering domain model. . . . . . . . . . . . . . . . 109

5.6 Illustration of the passivity compensation process. . . . . . . . . . 123

5.7 Eigenvalue spectrum of the FDGEP for example 1. . . . . . . . . . 128

5.8 Real eigenvalues of $\left[\boldsymbol{Y}^{H}(j \omega)+\boldsymbol{Y}(j \omega)\right]$ for example 1. . . . . . . . . 129 
5.9 Accuracy of the DRF macromodel for example 1 (the Original Tabulated Data, Original Nonpassive Model, and Passive Model are overlapping). . 130

5.10 Circuit for transient analysis in example 1. . . . . . . . . . . . 131

5.11 Transient response for $V_{\text {out }}(t)$ in example 1 (the Original and Passive Model are overlapping). . . . . . . . . . . . . . 132

5.12 Accuracy of the DRF macromodel for example 2 (the Original Tabulated Data and Original Passive Model are overlapping). . . . . . . . . . . 133

5.13 Eigenvalue spectrum of the FDGEP for example 2. . . . . . . . . . . . . 134

5.14 Real eigenvalues of $\left[\boldsymbol{Y}^{H}(j \omega)+\boldsymbol{Y}(j \omega)\right]$ for example 2. . . . . . . . . . 134

5.15 Telecommunication switching circuit link for example 3. . . . . . . 135

5.16 Eigenvalue spectrum of the FDGEP for example 3. . . . . . . . . . . . 135

5.17 Real eigenvalues of $\left[\boldsymbol{Y}^{H}(j \omega)+\boldsymbol{Y}(j \omega)\right]$ for example 3. . . . . . . . . 136

5.18 Expanded view of eigenvalues for example 3 on the interval $[0,0.7870] \times$ $10^{-9} \mathrm{GHz} . \ldots \ldots \ldots 137$

5.19 An illustration of the DRF approximation for example 3 (the Original Tabulated Data, Original Nonpassive Model, and Passive Model are all overlapping

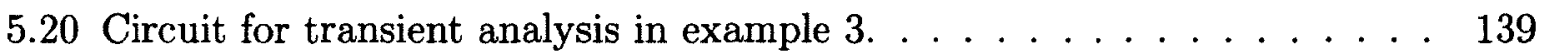

5.21 Transient response for $V_{\text {out }}(t)$ in example $3 . \ldots \ldots$. . . . . . . . . 139

6.1 Example of a system with multiple periodic delayed responses from an internal reflection.

6.2 A sample comparison of the scattering parameters, $Y_{21}(j \omega)$, for example 1. .......................... 150

6.3 Circuit for transient analysis of example 1. . . . . . . . . . . . 151

6.4 Comparison of transient responses for example 1 (all the responses overlap). 152

6.5 A sample comparison of the scattering parameters, $S_{22}(j \omega)$, for example 2 . 
6.6 Circuit for transient analysis of example 2. . . . . . . . . . . 155

6.7 Comparison of transient responses for example 2 (all the responses overlap). 155

6.8 Eye diagrams for example $2 \ldots \ldots \ldots \ldots$

7.1 RLC circuit with an embedded subnetwork described by a time-delay de-

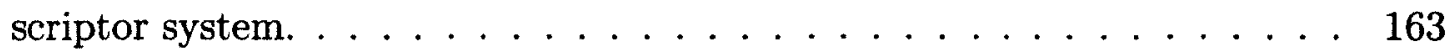

7.2 Original network for example 1. . . . . . . . . . . . 175

7.3 A sample spectrum of admittance parameters for example 1 (all the responses overlap). . . . . . . . . . . . . . . 176

7.4 Eigenvalue spectrum of admittance parameters for example 1. . . . . 176

7.5 A comparison of transient responses for example 1. . . . . . . . . . 177

7.6 Original network for example $2 \ldots \ldots \ldots \ldots$. . . . . . . . 178

7.7 A sample spectrum of admittance parameters for example 2 (all the responses overlap). . . . . . . . . . . . . . . . . 179

7.8 A sample eigenvalue spectrum of admittance parameters for example 2. . 180

7.9 A comparison of transient responses for example 2. . . . . . . . . . 180 


\section{List of Abbreviations}

AWE ....... Asymptotic Waveform Evaluation

CAD . . . . . . . Computer-Aided Design

CFH . . . . . . . Complex Frequency Hopping

CPU . . . . . . . . Central Processing Unit

DRF . . . . . . . Delayed Rational Function

EM . . . . . . Electro-Magnetic

EMI . . . . . . . . Electro-Magnetic Interference

FDGEP . . . . . . . F Frequency-Dependent Generalized Eigenvalue Problem

IC . . . . . . . . Interconnect Circuit

IFFT . . . . . . Inverse Fast Fourier Transform

MCM . . . . . . . Multi-Chip Modules

MEM . . . . . . . Micro-Electro-Mechanical

MNA . . . . . . . Modified Nodal Analysis

ODE . . . . . . Ordinary Differential Equation

PCB . . . . . . . Printed Circuit Board

PMTBR . . . . . . . Poor Man's Truncated Balanced Realization

PRIMA . . . . . . Passive Reduced-Order Interconnect Macromodeling Algorithm

RAM . . . . . R Random-Access Memory 
RLCG . . . . . . . . Resistance, Inductance, Capacitance, and Conductance RMSE . . . . . . . Root Mean Square Error

SPICE . . . . . . . Simulation Program with Integrated Circuit Emphasis TDD . . . . . . . . Time-Delay Descriptor

VLSI . . . . . . . . Very Large Scale Integration 


\section{List of Symbols}

$\boldsymbol{A} \ldots \ldots$ Matrix A

o . . . . . . Matrix of zeros

I . . . . . Identity matrix

$\boldsymbol{I}_{n} \ldots \ldots \ldots$. Identity matrix of size $n \times n$

$\boldsymbol{A}^{T} \ldots \ldots$. . . Transpose of the matrix $\boldsymbol{A}$

$\boldsymbol{A}^{*} \ldots \ldots$ Complex conjugate of the matrix $\boldsymbol{A}$

$\boldsymbol{A}^{H} \ldots \ldots \ldots$ Hermitian conjugate of the matrix $\boldsymbol{A}$

$\mathbb{R} \ldots \ldots \ldots$ The set of all real numbers

$\mathbb{R}^{N} \ldots \ldots \ldots$ The set of all real vectors of size $N$

$\mathbb{R}^{N \times M} \ldots \ldots$ The set of all real matrices of size $N \times M$

C . . . . . . . . The set of all complex numbers

$\mathbb{C}^{N} \ldots \ldots \ldots$ The set of all complex vectors of size $N$

$\mathbb{C}^{N \times M} \ldots \ldots \ldots$ The set of all complex matrices of size $N \times M$

$\mathfrak{R e}(x) \ldots \ldots$. Real part of the complex number $x$

$\mathfrak{I m}(x) \ldots \ldots$ Imaginary part of the complex number $x$

$j \ldots \ldots \ldots \cdot \sqrt{-1}$

$\arg (\phi) \ldots \ldots \ldots$ Argument or phase angle of the complex value $\phi$

$\otimes \ldots \ldots \ldots$ Kronecker product operator

$\mathfrak{L} \ldots \ldots \ldots$. . . . Laplace operator 
$\omega \ldots \ldots \ldots$ Angular frequency

$s \ldots \ldots \ldots$. Independent variable of the Laplace-domain

$|x| \ldots \ldots \ldots$ Absolute value of $x$

$\|\boldsymbol{A}\| \ldots \ldots$. . . . . . . of the matrix $\boldsymbol{A}$

$\operatorname{vec}(\boldsymbol{A}) \ldots \ldots$. The rows of $\boldsymbol{A}$ stacked in vector format 


\section{CHAPTER 1}

\section{Introduction}

\subsection{Background and Motivation}

With the continuing demand for more functionality, electronic designs require an ever-increasing integration of diverse devices such as optical components, analog circuits, digital blocks, and Micro-Electro-Mechanical (MEM) systems. Coupled with higher operating speeds, sharper excitations, and denser circuit layouts, ensuring signal integrity of electronic modules has become a challenging task. High-frequency effects such as reflection, crosstalk, delays, attenuation and Electro-Magnetic Interference (EMI) are critically effecting the signal propagation behavior [1]-[5]. Fig. 1.1 illustrates some of these issues. If inaccurately predicted, these effects can cause unexpected switching or logic glitches, which in turn can cause final products to malfunction or even fail. Therefore, due to the high cost of fabrication and product recalls, identification of signal integrity issues prior to manufacturing is essential. For this purpose, designers rely primarily on Computer-Aided Design (CAD) tools to accurately emulate signal performance at the modeling and simulation stage of design. 


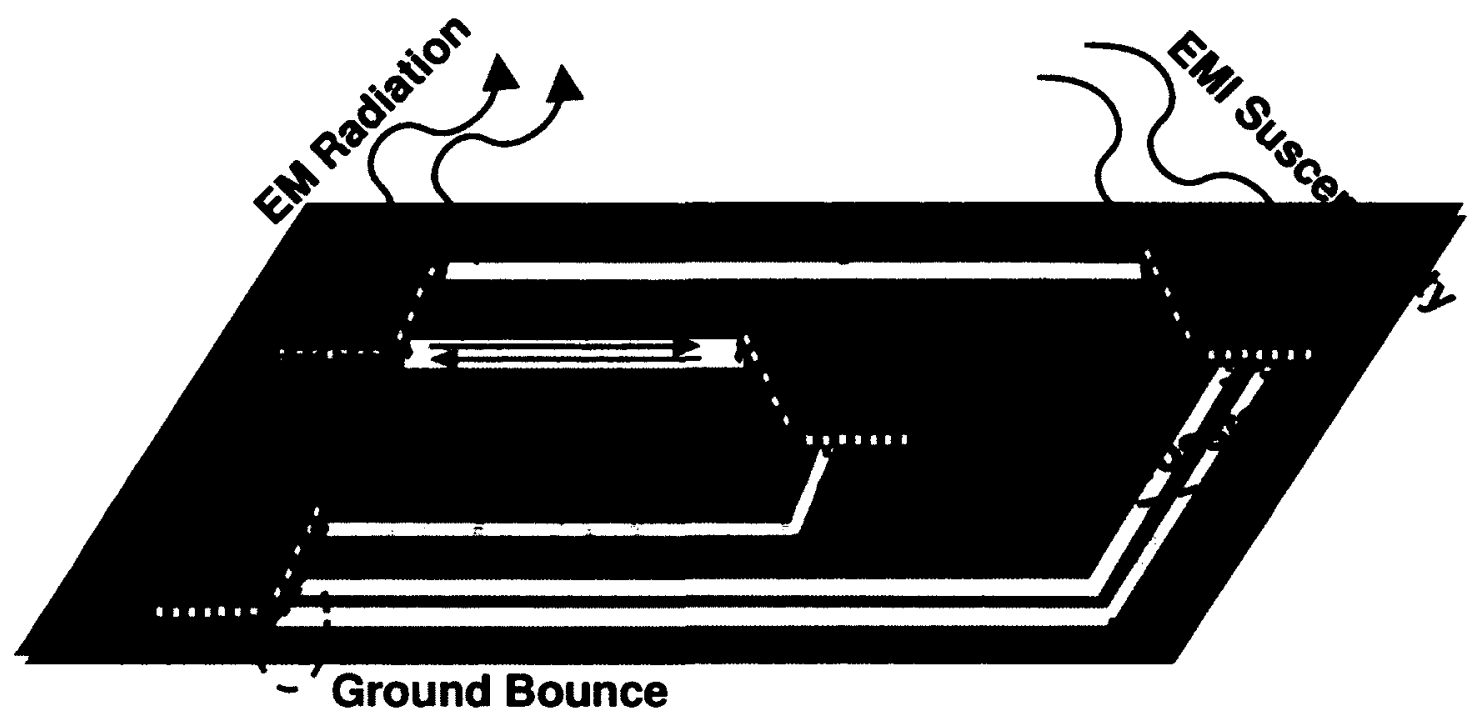

FIGURE 1.1: Illustration of signal integrity issues (PCB: Printed Circuit Board; IC: Interconnect Circuit).

This is achieved through rigorous time-domain analysis of each module, as well as the final overall design, using state-of-the-art SPICE simulators [5]-[8].

For the purpose of accurate time-domain simulation and analysis, each device requires a model that effectively characterizes its response over the desired operating bandwidth. In the past, low-order analytical approximations provided an acceptable level of detail to ensure proper functionality of the final product. However, the accuracy of these approximate models degrades at higher frequencies as the distributed nature of components becomes more prominent. A true analytical model requires a full solution to the partial differential equations (i.e. Maxwell's equations) that characterize the physical behavior of the device. With increasingly complex signal propagation paths and irregular structures, a full analytical solution to these partial differential equations is not always possible. For instance, interconnects in chip packages are usually nonuniform due to high circuit density, complex shapes and 
geometrical constraints at the edges of the chip. Numerous similar situations can be found, such as vias, nonuniform transmission lines, on-chip passive components and microwave devices, where analytical modeling becomes a difficult task. In such cases, the behavior of these modules is generally characterized by sampled/tabulated data, which can be in the admittance, impedance, scattering, or hybrid domains. The tabulated data can be obtained either directly from measurements or from rigorous full-wave 3D Electro-Magnetic (EM) field simulations [9]-[25]. Several advantages of tabulated data-based techniques are as follows. First, they have general and widespread applicability with the modeling of numerous electronic and microwave components such as Printed Circuit Boards (PCBs), nonuniform transmission lines, power systems, package structures, Multi-Chip Modules (MCMs), backplanes, cables, VLSI circuits, etc. Second, using tabulated data for modeling purposes does not require details of every internal component and circuit interconnection, which can be cumbersome to include in circuit simulators (or proprietary and may not be provided by the manufacturer). Third, the models can provide a high degree of accuracy over a large frequency bandwidth compared to low-order analytical approximations.

Integrating tabulated data models in standard SPICE simulators is a challenging task and has been a topic of intense research in recent years [9]-[25]. Standard circuit simulators are based on efficient time-domain solution techniques of Ordinary Differential Equations (ODEs), while tabulated data is typically provided in the frequency-domain [17]. Initial methodologies take a direct approach and convert the bandlimited tabulated frequency-domain data to time-domain data via an Inverse Fast Fourier Transform (IFFT). Subsequently, time-domain convolution is applied using the input excitations [9]-[11]. These techniques can be computationally expensive since a large number of data points is required to accurately capture high-frequency 
effects in the time-domain. In addition, if aliasing from the IFFT is not properly addressed, the overall time-domain results may be inaccurate. Recently, several techniques have been developed for the specific purpose of performing fast, accurate, and stable transient analysis of tabulated data networks. These new methodologies obtain an approximation for the tabulated data as a set of differential equations or macromodel. A key advantage of macromodels is that, they can easily be integrated in standard circuit simulators either directly as ODEs or by conversion to an equivalent circuit representation [5]. In addition, they can make use of the fast, accurate, and robust ODE solvers in a SPICE engine.

Conventional macromodeling approaches approximate the tabulated data using a set of rational functions (in the frequency-domain), which correspond to a set of ODEs in the time-domain. There are several algorithms available in the literature to obtain rational functions from either tabulated time-domain or frequency-domain data [13]-[25]. The most prominent of these techniques is vector fitting [17], which employs least squares methods to iteratively update the poles and residues of a rational approximation. Attempts to improve these rational fitting algorithms were focussed on increasing the accuracy and convergence of the related fitting algorithms, but have done little to reduce the size and efficiency of the resulting macromodels in transient simulators [18]-[24]. On the other hand, higher operating frequencies and longer system delays are resulting in extremely high-order rational approximations and subsequently, prohibitively large macromodels for transient simulation.

To illustrate the difficulties associated with long delay lines, consider the timedomain response of an electrically long interconnect in Fig. 1.2(a) [26]. The corresponding frequency-domain response is shown in Fig. 1.2(b). When this response is approximated using a direct rational approximation [17], the number of poles required 


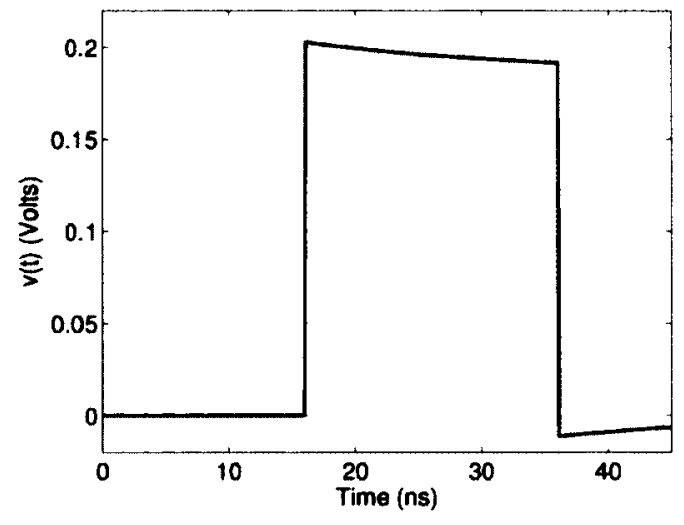

(a) Sample time-domain response.

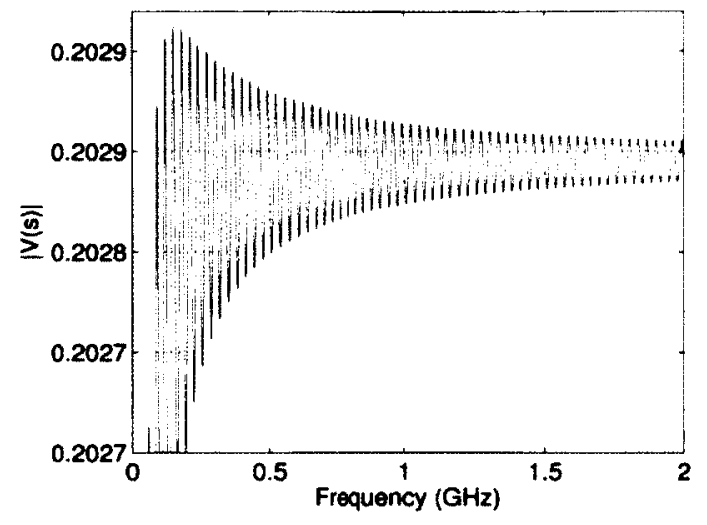

(b) Magnitude of the frequency-domain response.

FIGURE 1.2: Illustration of sample time and frequency-domain responses for a long delay line.

is very high ( 700 poles to fit up to $8 \mathrm{GHz}$ ). This is mainly because the waveform under consideration consists of a long delay. Consequently, the size of the macromodel is very high (typically given by the product of the number of ports and poles), leading to slow transient analysis using standard circuit simulators.

In order to overcome this issue, Delayed Rational Function (DRF) based approaches were introduced for electrically long networks characterized by tabulated data [26]-[34]. In [31]-[34] Hilbert transform based delay estimation techniques were proposed for general networks characterized by tabulated frequency-domain data. These methods extract only one dominant delay and neglect higher order reflections. Hence, the corresponding macromodel may not be efficient in the practical case of data with multiple delays. In [26], [27], general DRF-based macromodeling techniques were developed for networks characterized by tabulated time-domain data with multiple delays. In [28], a Gabor transform based macromodeling technique was proposed and $[29],[30]$ coupled this technique with a delayed version of the vector fitting algorithm. These methods were developed for networks with multiple delays characterized 
by tabulated frequency-domain data. Conceptually, algorithms in [26]-[30] are similar. Delays are first identified using time-frequency analysis and subsequently, a vector fitting type iterative least squares process is applied to obtain an accurate estimation of the poles and residues in the delayed rational terms.

A major drawback of the above type of DRF approximation techniques is that, they do not guarantee passivity. Macromodel passivity is critical to the stability of the transient simulation of the tabulated data network along with the rest of the circuitry. A stable, nonpassive model terminated with passive components can produce an overall unstable network. Consequently, the development of algorithms for macromodel passivity verification and enforcement is a topic of intense research [35]-[53]. It is to be noted that passivity verification is a computationally expensive step. Conventional verification requires a fine frequency-sweep of the entire right-half of the complex plane, and in many cases passivity violations may be missed if the sweep is too coarse. Widely adopted passivity enforcement methods may require numerous iterations, requiring at each iteration passivity verification and enforcement. In the case of rational function based macromodels, the above process is well established in the literature [35]-[49]. For passivity verification of rational function based macromodels, efficient and robust techniques based on evaluation of full-size and half-size Hamiltonian matrix eigenvalues can be found [35]-[39]. If the macromodels are found to be nonpassive, then several efficient compensation algorithms have been developed based on linear matrix inequalities and first-order perturbation theory [39]-[49].

For macromodels with time-delays, passivity verification and compensation has only been addressed for the specific case of transmission line models derived via the method of characteristics [50]-[53]. In these types of methods, passivity verification was formulated as a quasi-periodic Frequency-Dependent Generalized Eigenvalue 
Problem (FDGEP), which reduced the necessary search region from the entire righthalf of the complex plane to a single finite interval along the imaginary axis [50], [51]. For passivity compensation, techniques based on first-order perturbation theory have been developed that iteratively perturb the residues until the macromodel becomes passive [52], [53]. However, there are currently no passivity verification or compensation techniques available in the literature for DRF-based macromodels that are derived from tabulated data, characterizing general networks.

In addition to the above passivity issues, current DRF-based techniques may not provide optimal macromodels for systems that contain periodic reflections. Conceptually, algorithms in [26]-[30] approximate each reflection in the response using a separate delayed rational term. An illustration of this is shown in Fig. 1.3. As seen in the figure, in many cases the impulses corresponding to reflections will not attenuate rapidly and require dozens of delayed rational terms, with each term needing another set of poles and residues. For the specific case of transmission lines characterized by tabulated data, several approaches tackling this issue have been developed [54]-[60]. The work in [54], [55] incorporates delay estimation into the vector fitting algorithm using optimization. Multiple delays are taken into account via the modal decomposition of the propagation function matrix. In [56], [57], a transmission line specific delay embedded passive compact macromodel was developed to handle infinite reflections in tabulated data. In [59], [60], a formulation tackling a similar problem was presented. However, it is limited to the specific case of transmission lines and does not guarantee the stability of the resulting macromodel. For general tabulated data networks with periodic delays corresponding to internal reflections, an efficient compact macromodeling approach has not been developed and is still a challenging and open problem. 


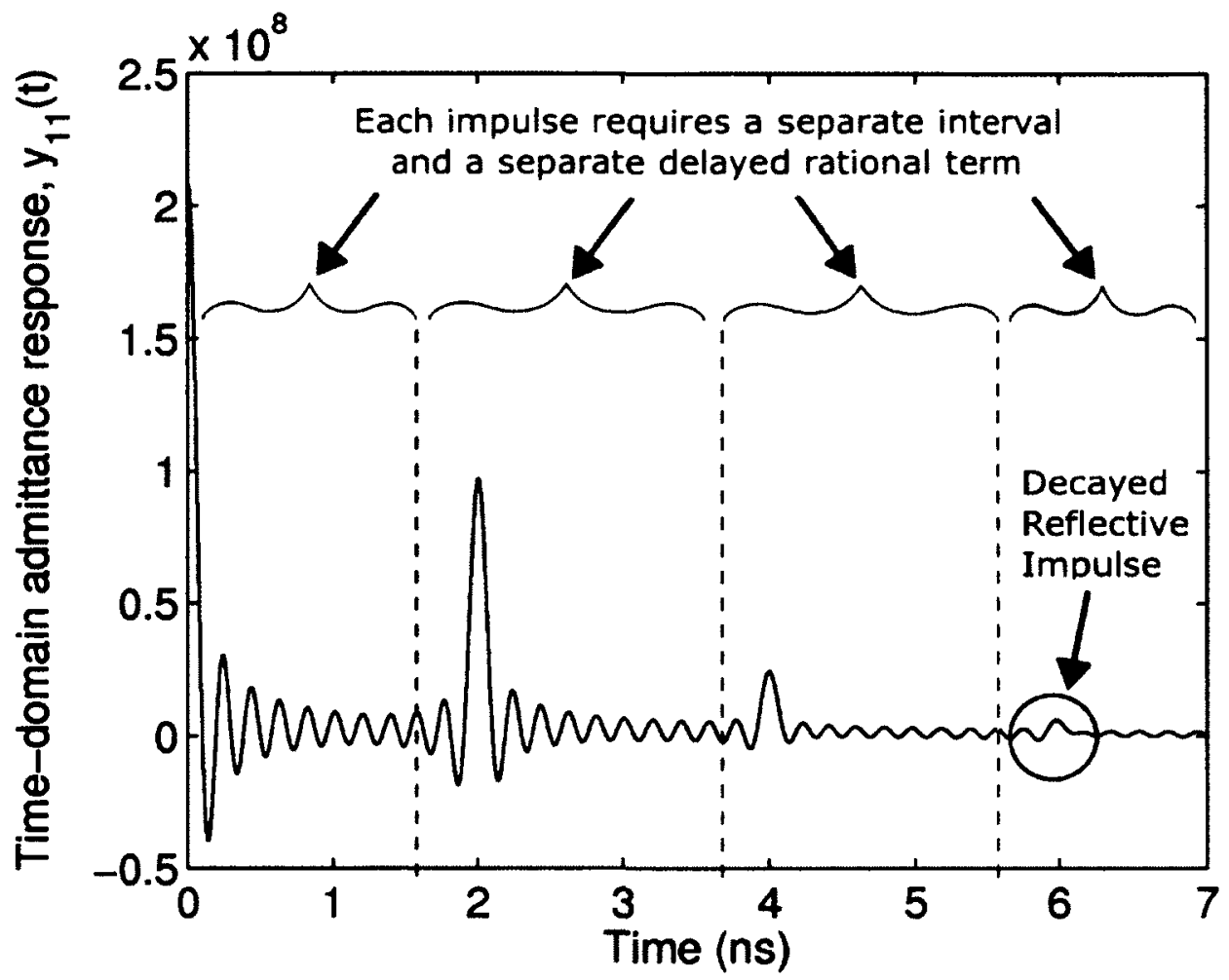

FIGURE 1.3: Illustration of periodic delays in the transient response and the corresponding requirements for a DRF approximation.

With increasing design complexity, unified global simulation and analysis has become a computationally expensive task that requires solving massive systems of differential equations. To accelerate the overall circuit analysis, model-order reduction techniques were introduced [7], [8], [61]-[64]. These techniques generate a reducedorder macromodel by projecting the large original system of equations into a lowerdimensional subspace that accurately retains the input-to-output relation of the original network. For large RLC circuit networks, Asymptotic Waveform Evaluation (AWE) was the first such reduction technique [7]. The reduced-order model is obtained such that the coefficients of the Taylor series expansion for its transfer function, or moments, match the moments of the original system. In [61], a Complex 
Frequency Hopping (CFH) algorithm was introduced for multi-point moment matching, which improved accuracy of the reduced-order macromodel over larger frequency bandwidths. A major drawback of the AWE and CFH algorithms is that, they do not guarantee the passivity of the reduced-order model. This issue was resolved in [8], for the specific case of RLC circuits, with the introduction of the Passive Reduced-Order Interconnect Macromodeling Algorithm (PRIMA). For general networks described by state-space equations, a passive reduction algorithm based on truncated balanced realization has also been developed [62]. However, these techniques are only applicable to systems of ordinary differential equations without time-delays.

As delay-based macromodels become a prominent tool for analyzing complex highspeed structures, they are increasingly incorporated in large modern circuit designs containing lumped RLC subnetworks, distributed interconnects, and general macromodels described in state-space form (without delays). Such networks are characterized by massive systems of delay differential equations and consequently, the above model-order reduction algorithms cannot be directly applied. Recently, for efficient unified transient analysis of large circuit networks with time-delays, two model-order reduction algorithms have been introduced [65], [66]. However in general, the passivity of these reduced-order macromodels is not guaranteed. Consequently, for RLC systems with embedded Time-Delay Descriptor (TDD) systems, passive model-order reduction remains a challenging task.

The goal of this thesis is to develop new algorithms for generating passive, compact, efficient, and accurate macromodels of electrically long high-speed networks. Specific contributions of this thesis are given in the next section. 


\subsection{Contributions}

The subject of this thesis is the passive macromodeling of high-speed networks with embedded time-delays. The algorithms presented here provide a means to guarantee accurate and efficient results from industry-grade circuit simulators using delay-based macromodels. The main contributions of this thesis are as follows.

1) New methods are developed for passivity verification of general DRF-based macromodels obtained from tabulated scattering and admittance parameter data [67]--[71]. Passivity verification is formulated as a quasi-periodic FDGEP, which only needs to be solved over a finite interval along the imaginary axis. The proposed approach allows for efficient and accurate identification and quantification of passivity violation regions for DRF-based macromodels.

2) A new passivity compensation algorithm is presented for general DRF-based macromodels obtained from tabulated scattering parameter data [68]-[70]. The proposed algorithm uses first-order perturbation theory to iteratively perturb the residues of the DRF macromodels. Necessary constraints are also enforced during the compensation process to minimize the error introduced in the response.

3) A new passivity compensation algorithm based on linear matrix inequalities is presented for general DRF-based macromodels obtained from tabulated admittance parameter data [67]. The proposed formulation can be solved efficiently using interior point methods.

4) A novel formulation is developed for macromodeling general networks characterized by tabulated data with long delays and periodic reflections [72]. The delays 
corresponding to periodic reflections are identified via time-frequency analysis and subsequently, the poles and residues are obtained using an iterative least squares process.

5) A new theorem is introduced for passivity verification of TDD systems [73]. The proposed theorem formulates passivity verification as a set of linear matrix inequalities.

6) A novel algorithm is developed for passive model-order reduction of RLC networks with embedded subnetworks described by TDD systems [73]. The proposed method formulates a unified system of equations describing the overall network such that, reduced-order models obtained via congruence transformbased model-order reduction techniques are guaranteed to be passive.

\subsection{Organization of the Thesis}

The remainder of this thesis is organized as follows. Chapter 2 presents a review of conventional passive macromodeling techniques for tabulated data. Chapter 3 reviews delay-based macromodeling techniques for tabulated data networks. Chapter 4 provides details of the new passivity verification and compensation techniques for DRF macromodels in the scattering domain. Next, Chapter 5 introduces techniques for passive DRF-based macromodeling in the admittance domain. In Chapter 6, a new and more efficient delay-extraction based macromodeling technique is developed for tabulated data networks with periodic delays. This is followed by Chapter 7, which presents a novel passive model-order reduction algorithm for RLC circuits with embedded subnetworks described by TDD systems. Chapter 8 summarizes the 
proposed work and outlines the direction of future research. 


\section{CHAPTER 2}

\section{Review of Modeling Techniques for Tabulated Data Networks}

Various approaches for modeling and simulating tabulated data networks are available in the literature. Selecting the correct approach for a specific application and network type is key for efficient analysis. This chapter provides an overview of some of the relevant methods for analysis of tabulated data networks. The chapter is organized as follows. Section 2.1 provides a review of conventional modeling and simulation techniques for tabulated data. Next, Section 2.2 discusses rational fitting techniques for tabulated data networks. Section 2.3 discusses stability, causality, and passivity issues with respect to macromodeling. This is followed by Section 2.4 , that reviews passivity verification and compensation techniques for rational function based macromodels [42]. 


\subsection{Review of Simulation and Modeling for Tabu-}

\section{lated Data Networks}

As it becomes increasingly difficult to obtain analytical models for high-speed modules, networks characterized by tabulated data are fast becoming an integral part of accurate signal integrity analysis in high-speed designs. The tabulated multi-port data for an $L$-port network is typically given at $N$ points in the frequency-domain as

$$
\boldsymbol{H}(s)=\left[H_{i k}(s)\right] ; \quad H_{i k}(s)=\left\{H_{i k}\left(s_{1}\right), H_{i k}\left(s_{2}\right), \ldots, H_{i k}\left(s_{N}\right)\right\}
$$

where $s_{n}=j 2 \pi f_{n},\left\{f_{1}, f_{2}, \ldots, f_{N}\right\}$ are the frequencies, and $(i, k) \in(1,2, \ldots, L)$. For the purpose of transient analysis, it is necessary to integrate these networks into standard SPICE simulators. However, SPICE simulators are based on solving systems of differential equations in the time-domain, while the tabulated data is given in the frequency-domain. Initial methods converted the tabulated data into the time-domain via IFFT and subsequently, convolved the time-domain impulse response data (from the IFFT) with the input excitation to perform transient analysis [9]-[11]. However, direct convolution-based approaches suffer from several drawbacks. First, they are based on the IFFT, which suffers from aliasing and is not always accurate. Second, for higher frequencies the IFFT produces an increasing number of time-domain samples. Consequently, the transient analysis can become very slow.

In order to overcome the above issues, techniques based on obtaining a set of differential equations or macromodel that accurately characterize the tabulated data over the operating bandwidth were introduced. Using these approaches, the tabulated data is approximated with a rational function in the frequency-domain [13]-[25]. Subse- 
quently, the rational functions are integrated in SPICE simulators either directly (for example, HSPICE has the Laplace-element [74]) or by converting the rational functions to an equivalent circuit [5]. Transient analysis can then be performed efficiently in SPICE via recursive convolution [12] or using standard numerical techniques for differential equations (such as Euler's method) [75].

A major requirement to obtaining accurate results from the rational models in the time-domain is that, the rational approximations in the frequency-domain accurately match the tabulated data. These approaches are based on either least squares fitting techniques [13]-[24] or interpolation [25]. A review of rational approximation techniques relevant to this thesis is provided in the following section.

\subsection{Rational Fitting Techniques}

To obtain a macromodel from a set of tabulated transfer function data given at $N$ frequency points, $\left\{f\left(s_{1}\right), f\left(s_{2}\right), \ldots, f\left(s_{N}\right)\right\}$, the data is first fit in the frequencydomain using a rational function of the form

$$
f(s)=\frac{a_{0}+a_{1} s^{1}+a_{2} s^{2}+\ldots+a_{p-1} s^{p-1}+a_{p} s^{p}}{1+b_{1} s^{1}+b_{2} s^{2}+\ldots+b_{q-1} s^{q-1}+b_{q} s^{q}}
$$

Here, the coefficients $a_{0}, a_{1}, \ldots, a_{p}$ and $b_{1}, b_{2}, \ldots, b_{q}$ are the unknowns that need to be determined. Solving for the coefficients in (2.2) directly is a nonlinear problem. However, this formulation can be linearized by multiplying both sides with the denominator term to give

$$
a_{0}+a_{1} s^{1}+a_{2} s^{2}+\ldots+a_{p} s^{p}-f(s) b_{1} s^{1}-f(s) b_{2} s^{2}-\ldots-f(s) b_{q} s^{q}=f(s)
$$


Then, to determine the unknown coefficients $\left\{a_{i}\right\}$ and $\left\{b_{i}\right\}$, a standard least squares problem in the form $\boldsymbol{A x}=\boldsymbol{b}$ is constructed. The expanded system is given by

$$
\overbrace{\left[\begin{array}{ccccccccc}
1 & s_{1} & s_{1}^{2} & \cdots & s_{1}^{p} & -f\left(s_{1}\right) s_{1} & -f\left(s_{1}\right) s_{1}^{2} & \cdots & -f\left(s_{1}\right) s_{1}^{q} \\
1 & s_{2} & s_{2}^{2} & \cdots & s_{2}^{p} & -f\left(s_{2}\right) s_{2} & -f\left(s_{2}\right) s_{2}^{2} & \cdots & -f\left(s_{2}\right) s_{2}^{q} \\
\vdots & \vdots & \ddots & \vdots & \vdots & \vdots & \vdots & \ddots & \vdots \\
1 & s_{N} & s_{N}^{2} & \cdots & s_{N}^{p} & -f\left(s_{N}\right) s_{N} & -f\left(s_{N}\right) s_{N}^{2} & \cdots & -f\left(s_{N}\right) s_{N}^{q}
\end{array}\right]}^{\boldsymbol{A}} \boldsymbol{x}=\overbrace{\left[\begin{array}{c}
f\left(s_{1}\right) \\
f\left(s_{2}\right) \\
\vdots \\
f\left(s_{N}\right)
\end{array}\right]}^{\boldsymbol{b}}
$$

with $\boldsymbol{x}$ containing the unknown coefficients

$$
\boldsymbol{x}=\left[\begin{array}{lllllllll}
a_{0} & a_{1} & a_{2} & \cdots & a_{p} & b_{1} & b_{2} & \cdots & b_{q}
\end{array}\right]^{T}
$$

Several fitting methods have been proposed based on solving this least squares formulation [13]-[16]. However, for high-order rational approximations and large frequency bandwidths, the least squares problem can become ill-conditioned. To overcome this issue, numerous methods have been developed. In [13], an iterative scheme was introduced, in which the solution of (2.4) is used in the first iteration to determine an approximation for all the coefficients. Subsequent iterations use the denominator from the previous iteration to improve the scaling and convergence of the fitting process (a detailed analysis this asymptotic behavior can be found in [76]). The methods in [14], [15] attempt to improve the conditioning by applying various scaling schemes. In [16], techniques based on a scaled iterative least squares problem and nonlinear optimization were developed. While these methods all provide improvements over directly solving the least squares problem in (2.4), they are not capable of handling high-order rational functions. 
Recently, a major breakthrough in rational approximation methods was made with the introduction of the vector fitting algorithm [17]. Instead of (2.2), the rational approximation is formulated as

$$
f(s)=\sum_{m=1}^{M} \frac{r_{m}}{s-p_{m}}+d+s h
$$

where $r_{m}$ and $p_{m}$ are real or come in complex conjugate pairs representing the residues and poles, respectively. The values $d$ and $h$ are both real, and $M$ is the number of poles. The first step in this technique is to make an initial guess at the poles. Next, the residues are determined via a least squares problem. Subsequently, the process in [13] is applied to iteratively update the poles and residues. Since (2.6) is linear in $s$ (i.e. does not contain $s^{p}$ and $s^{q}$ terms) the least squares problem is well-conditioned and accurate solutions can be obtained for high-order rational functions and large frequency-bandwidths.

Numerous modifications and improvements to the vector fitting procedure have been introduced [18]-[24]. A detailed review of the vector fitting algorithm and some of these modifications is presented in the following section.

\subsubsection{The Vector Fitting Algorithm}

The vector fitting algorithm [17] begins with a set of $N$ tabulated data points, $\left\{f\left(s_{1}\right), f\left(s_{2}\right), \ldots, f\left(s_{N}\right)\right\}$ and builds a rational function approximation in the form of (2.6). The goal of the algorithm is to estimate all the coefficients in (2.6) so that the rational function, $f(s)$, provides an accurate approximation to the data. To this end, (2.6) is solved through a linear formulation using two stages. Stage one makes an initial guess at the poles over the frequency interval of interest. Next, it iterates 
on the initial guess of the poles by solving a least squares problem for an unknown function, $\sigma(s)$, whose zeros become the new poles of the system. Stage two then calculates the residues of (2.6) using the final set of poles from stage one.

First, the details of the algorithm using real poles are reviewed. Following this, a robust choice of starting poles are defined and modifications for complex poles and multi-port networks are examined. Subsequently, details for improving the convergence of vector fitting when using noisy data sets are reviewed.

\section{Stage 1: Pole Identification}

The algorithm begins by choosing a set of starting poles, $\left\{\bar{p}_{m}\right\}$. Following this, a scaling function, $\sigma(s)$, is defined such that

$$
\sigma(s)=\sum_{m=1}^{M} \frac{\tilde{r}_{m}}{s-\bar{p}_{m}}+1
$$

where $\tilde{r}_{m}$ are the residues of $\sigma(s)$. Next, a scaled function is defined such that

$$
\sigma(s) f(s) \approx \hat{f}(s)=\sum_{m=1}^{M} \frac{\hat{r}_{m}}{s-\bar{p}_{m}}+\hat{d}+s \hat{h}
$$

Equations (2.7) and (2.8) are combined to obtain

$$
\left[\sum_{m=1}^{M} \frac{\hat{r}_{m}}{s-\bar{p}_{m}}+\hat{d}+s \hat{h}\right] \approx\left[\sum_{m=1}^{M} \frac{\tilde{r}_{m}}{s-\bar{p}_{m}}+1\right] f(s)
$$

or

$$
\left[\sum_{m=1}^{M} \frac{\hat{r}_{m}}{s-\bar{p}_{m}}+\hat{d}+s \hat{h}\right]-\left[\sum_{m=1}^{M} \frac{\tilde{r}_{m}}{s-\bar{p}_{m}}\right] f(s)=f(s) .
$$


Writing (2.10) at $N \geq(2 M+2)$ frequency points (where $2 M+2$ is the number of unknowns) gives

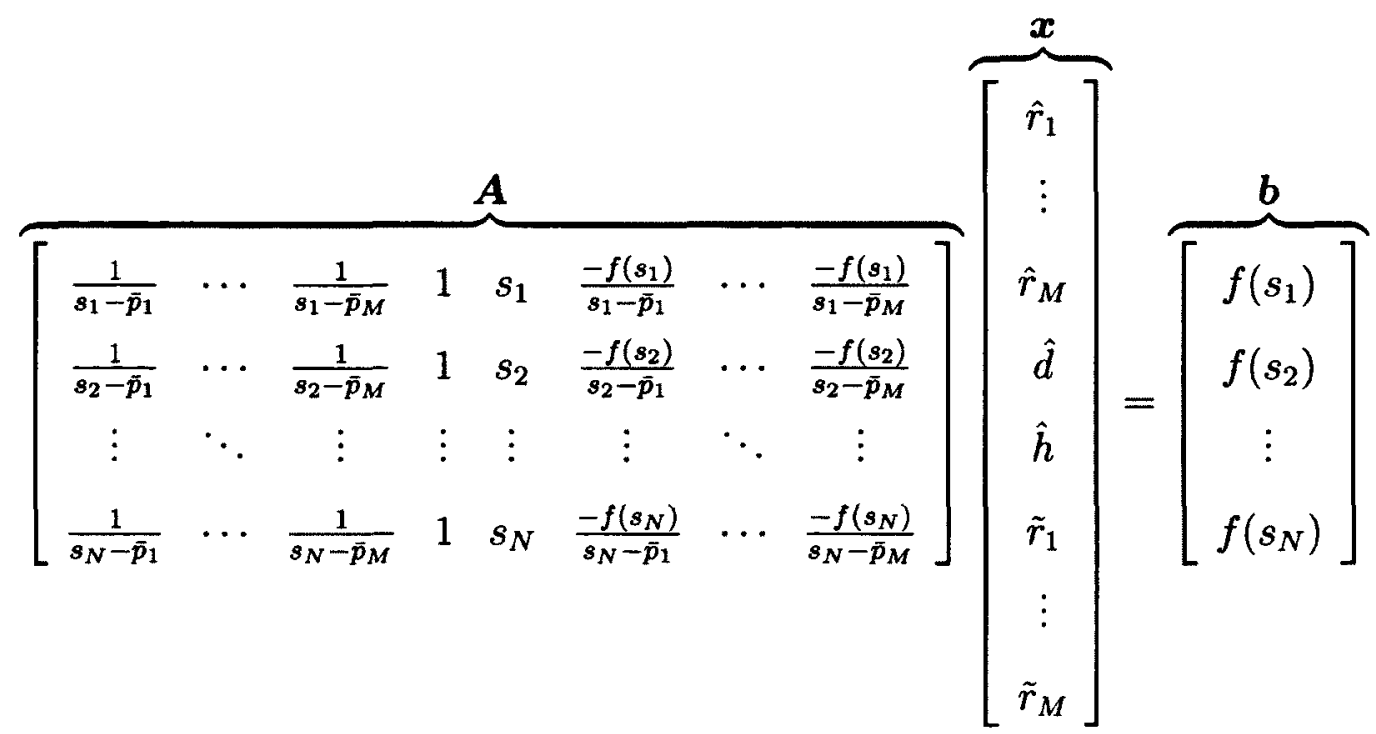

where all the unknowns $\hat{r}_{m}, \hat{d}, \hat{h}$, and $\tilde{r}_{m}$ are real provided the poles are real. This is an overdetermined least squares problem that can be solved efficiently using the standard Householder algorithm [77].

Equation (2.10) is now used to iteratively obtain a rational function approximation for $f(s)$. Let $\left\{\hat{z}_{m}\right\}$ and $\left\{\tilde{z}_{m}\right\}$ be the zeros of $\hat{f}(s)$ and $\sigma(s)$, respectively. Then the partial fraction expansion of $\sigma(s)$ and $f(s)$ can be written as

$$
\hat{f}(s)=\hat{h} \frac{\prod_{m=1}^{M+1}\left(s-\hat{z}_{m}\right)}{\prod_{m=1}^{M}\left(s-\bar{p}_{m}\right)}, \quad \sigma(s)=\frac{\prod_{m=1}^{M}\left(s-\tilde{z}_{m}\right)}{\prod_{m=1}^{M}\left(s-\bar{p}_{m}\right)} .
$$


Substituting (2.12) into (2.8) yields

$$
f(s)=\frac{\hat{f}(s)}{\sigma(s)}=\hat{h} \frac{\left(\prod_{m=1}^{M+1}\left(s-\hat{z}_{m}\right)\right) /\left(\prod_{m=1}^{M}\left(s-\bar{p}_{m}\right)\right)}{\left(\prod_{m=1}^{M}\left(s-\tilde{z}_{m}\right)\right) /\left(\prod_{m=1}^{M}\left(s-\bar{p}_{m}\right)\right)}=\hat{h} \frac{\left(\prod_{m=1}^{M+1}\left(s-\hat{z}_{m}\right)\right)}{\left(\prod_{m=1}^{M}\left(s-\tilde{z}_{m}\right)\right)} .
$$

Therefore, the zeros of $\sigma(s)$ represent a refined set of poles for the function $f(s)$. This stage is repeated with the zeros of $\sigma(s)$ taken as the new set of starting poles until the desired convergence criteria is obtained.

In some instances, the poles determined using (2.13) will lie in the right-half plane, meaning they are unstable. This problem is overcome by inverting the signs of the real parts for the unstable poles and performing another iteration.

Calculation of Zeros: The zeros of the function, $\sigma(s)$ can also be obtained by determining the eigenvalues of the matrix [17]

$$
\boldsymbol{H}=\Psi-\boldsymbol{\Psi} \tilde{\phi}^{T}
$$

where

$$
\boldsymbol{\Psi}=\left[\begin{array}{cccc}
\bar{p}_{1} & 0 & \cdots & 0 \\
0 & \bar{p}_{2} & \ddots & \vdots \\
\vdots & \ddots & \ddots & 0 \\
0 & \cdots & 0 & \bar{p}_{M}
\end{array}\right], \quad \boldsymbol{g}=\left[\begin{array}{c}
1 \\
1 \\
\vdots \\
1
\end{array}\right]_{M \times 1}, \quad \tilde{\boldsymbol{\phi}}^{T}=\left[\begin{array}{llll}
\tilde{r}_{1} & \tilde{r}_{2} & \cdots & \tilde{r}_{M}
\end{array}\right]_{1 \times M}
$$

The matrix $\Psi$ is a diagonal matrix containing the starting poles, $g$ is a vector of ones, and $\tilde{\phi}^{T}$ is a row vector containing the residues of $\sigma(s)$. 


\section{Stage 2: Residue Identification}

In this stage, the residues of $f(s)$ are calculated using the final set of poles, $\left\{p_{i}\right\}$, found in stage one. For this purpose, an overdetermined least squares problem is constructed that solves for the residues in (2.6) at $N$ frequency points. Expanding this in the form $\boldsymbol{A x}=\boldsymbol{b}$ gives

$$
\overbrace{\left[\begin{array}{ccccc}
\frac{1}{s_{1}-p_{1}} & \cdots & \frac{1}{s_{1}-p_{M}} & 1 & s_{1} \\
\frac{1}{s_{2}-p_{1}} & \cdots & \frac{1}{s_{2}-p_{M}} & 1 & s_{2} \\
\vdots & \ddots & \vdots & \vdots & \vdots \\
\frac{1}{s_{N}-p_{1}} & \cdots & \frac{1}{s_{N}-p_{M}} & 1 & s_{N}
\end{array}\right]}^{\boldsymbol{A}} \overbrace{\left[\begin{array}{c}
r_{1} \\
\vdots \\
r_{M} \\
d \\
h
\end{array}\right]}^{\boldsymbol{x}}=\overbrace{\left[\begin{array}{c}
f\left(s_{1}\right) \\
f\left(s_{2}\right) \\
\vdots \\
f\left(s_{N}\right)
\end{array}\right]}^{\boldsymbol{b}}
$$

\section{Selection of Starting Poles}

Functions with distinct resonance peaks should be modeled using complex poles. In this case, the initial set of poles for the algorithm consists of imaginary parts, $\beta$, linearly distributed over the frequency range of interest. These poles are chosen [17] in complex conjugate pairs such that

$$
p_{i}=-\alpha+j \beta, \quad p_{i+1}=-\alpha-j \beta,
$$

where

$$
\alpha=\beta / 100
$$

is chosen to be small to avoid ill-conditioning in the least squares problems [17].

Smooth functions are best modeled using real poles linearly or logarithmically 
spaced as a function of frequency.

\section{Modifications for Complex Poles}

In order to ensure that the residues from the least squares computations in (2.11) and (2.16) come in exact complex conjugate pairs, the matrix formulations are modified. If the partial fractions $i$ and $i+1$ correspond to complex pole pairs, then we have $\frac{M}{2}$ complex pairs where

$$
\begin{aligned}
p_{i} & =\mathfrak{R e}\left(p_{i}\right)+j \mathfrak{I m}\left(p_{i}\right), & p_{i+1} & =\mathfrak{R e}\left(p_{i}\right)-j \mathfrak{I m}\left(p_{i}\right), \\
r_{i} & =\mathfrak{R e}\left(r_{i}\right)+j \mathfrak{I m}\left(k_{i}\right), & r_{i+1} & =\mathfrak{R e}\left(r_{i}\right)-j \mathfrak{I m}\left(r_{i}\right) .
\end{aligned}
$$

Also, the overdetermined least squares problem $\boldsymbol{A x}=\boldsymbol{b}$ in (2.11) becomes

$$
\begin{aligned}
& \boldsymbol{A}=\left[\begin{array}{llllll}
\boldsymbol{A}_{1} & \boldsymbol{A}_{2} & \cdots & \boldsymbol{A}_{\frac{M}{2}} & \mathbf{1} & \boldsymbol{S}
\end{array}\right], \quad \boldsymbol{b}=\left[\begin{array}{lllll}
f\left(s_{1}\right) & f\left(s_{2}\right) & \cdots & f\left(s_{N}\right)
\end{array}\right]^{T}, \\
& \boldsymbol{x}=\left[\mathfrak{R e}\left(\hat{r}_{1}\right) \mathfrak{I m}\left(\hat{r}_{1}\right) \mathfrak{R e}\left(\tilde{r}_{1}\right) \mathfrak{I m}\left(\tilde{r}_{1}\right) \ldots \mathfrak{R e}\left(\hat{r}_{\frac{M}{2}}\right) \mathfrak{I m}\left(\hat{r}_{\frac{M}{2}}\right) \mathfrak{R e}\left(\tilde{r}_{\frac{M}{2}}\right) \mathfrak{I m}\left(\tilde{r}_{\frac{M}{2}}\right) \hat{d} \hat{h}\right]^{T}
\end{aligned}
$$

with the submatrices of $\boldsymbol{A}$ defined by

$$
\boldsymbol{A}_{i}=\left[\begin{array}{cccc}
\left(\frac{1}{s_{1}-\bar{p}_{i}}+\frac{1}{s_{1}-\bar{p}_{i}^{*}}\right) & \left(\frac{j}{s_{1}-\bar{p}_{i}}-\frac{j}{s_{1}-\bar{p}_{i}^{*}}\right) & -\left(\frac{f\left(s_{1}\right)}{s_{1}-\bar{p}_{i}}+\frac{f\left(s_{1}\right)}{s_{1}-\bar{p}_{i}^{*}}\right) & -\left(\frac{j f\left(s_{1}\right)}{s_{1}-\bar{p}_{i}}-\frac{j f\left(s_{1}\right)}{s_{1}-\bar{p}_{i}^{*}}\right) \\
\left(\frac{1}{s_{2}-\bar{p}_{i}}+\frac{1}{s_{2}-\bar{p}_{i}^{*}}\right) & \left(\frac{j}{s_{2}-\bar{p}_{i}}-\frac{j}{s_{2}-\bar{p}_{i}^{*}}\right) & -\left(\frac{f\left(s_{2}\right)}{s_{2}-\bar{p}_{i}}+\frac{f\left(s_{2}\right)}{s_{2}-\bar{p}_{i}^{*}}\right) & -\left(\frac{j f\left(s_{2}\right)}{s_{2}-\bar{p}_{i}}-\frac{j f\left(s_{2}\right)}{s_{2}-\bar{p}_{i}^{*}}\right) \\
\vdots & \vdots & \vdots \\
\left(\frac{1}{s_{N}-\bar{p}_{i}}+\frac{1}{s_{N}-\bar{p}_{i}^{*}}\right) & \left(\frac{j}{s_{N}-\bar{p}_{i}}-\frac{j}{s_{N}-\bar{p}_{i}^{*}}\right) & -\left(\frac{f\left(s_{N}\right)}{s_{N}-\bar{p}_{i}}+\frac{f\left(s_{N}\right)}{s_{N}-\bar{p}_{i}^{*}}\right)-\left(\frac{j f\left(s_{N}\right)}{s_{N}-\bar{p}_{i}}-\frac{j f\left(s_{N}\right)}{s_{N}-\bar{p}_{i}^{*}}\right)
\end{array}\right],
$$


Similarly, the terms $\boldsymbol{A}, \boldsymbol{x}$, and $\boldsymbol{b}$ from the residue identification problem in (2.16) become

$$
\begin{aligned}
& \boldsymbol{A}=\left[\begin{array}{llllll}
\boldsymbol{A}_{1} & \boldsymbol{A}_{2} & \cdots & \boldsymbol{A}_{\frac{M}{2}} & \mathbf{1} & \boldsymbol{S}
\end{array}\right], \quad \boldsymbol{b}=\left[\begin{array}{lllll}
f\left(s_{1}\right) & f\left(s_{2}\right) & \cdots & f\left(s_{N}\right)
\end{array}\right]^{T}, \\
& \boldsymbol{x}=\left[\begin{array}{lllllll}
\mathfrak{R e}\left(r_{1}\right) & \mathfrak{I m}\left(r_{1}\right) & \cdots & \mathfrak{R e}\left(r_{\frac{M}{2}}\right) & \mathfrak{I m}\left(r_{\frac{M}{2}}\right) & d & h
\end{array}\right]^{T}
\end{aligned}
$$

with

$$
\boldsymbol{A}_{i}=\left[\begin{array}{cc}
\left(\frac{1}{s_{1}-\bar{p}_{i}}+\frac{1}{s_{1}-\bar{p}_{i}^{*}}\right) & \left(\frac{j}{s_{1}-\bar{p}_{i}}-\frac{j}{s_{1}-\bar{p}_{i}^{*}}\right) \\
\left(\frac{1}{s_{2}-\bar{p}_{i}}+\frac{1}{s_{2}-\bar{p}_{i}^{*}}\right) & \left(\frac{j}{s_{2}-\bar{p}_{i}}-\frac{j}{s_{2}-\bar{p}_{i}^{*}}\right) \\
\vdots & \vdots \\
\left(\frac{1}{s_{N}-\bar{p}_{i}}+\frac{1}{s_{N}-\bar{p}_{i}^{*}}\right) & \left(\frac{j}{s_{N}-\bar{p}_{i}}-\frac{j}{s_{N}-\bar{p}_{i}^{*}}\right)
\end{array}\right], \quad \mathbf{1}=\left[\begin{array}{c}
1 \\
1 \\
\vdots \\
1
\end{array}\right]_{N \times 1}, \quad \boldsymbol{S}=\left[\begin{array}{c}
s_{1} \\
s_{2} \\
\vdots \\
s_{N}
\end{array}\right] .
$$

To ensure poles and residues occur in exact complex conjugate pairings, the least squares problems in (2.21) and (2.23) are formulated in terms of real quantities as

$$
\overbrace{\left[\begin{array}{l}
\mathfrak{R e}(\boldsymbol{A}) \\
\mathfrak{I m}(\boldsymbol{A})
\end{array}\right]}^{\overline{\boldsymbol{A}}} \boldsymbol{x}=\overbrace{\left[\begin{array}{l}
\mathfrak{R e}(\boldsymbol{b}) \\
\mathfrak{I m}(\boldsymbol{b})
\end{array}\right]}^{\overline{\boldsymbol{b}}} .
$$

Calculation of Zeros for Complex Poles: In the case of complex poles, the calculation of zeros from (2.14) needs to be modified as well. The zeros are now the eigenvalues of the system

$$
\boldsymbol{H}=\boldsymbol{\Psi}-\boldsymbol{g} \tilde{\phi}^{T}
$$


where

$$
\begin{aligned}
& \boldsymbol{\Psi}=\left[\begin{array}{cccc}
\boldsymbol{\Psi}_{1} & 0 & \cdots & 0 \\
0 & \boldsymbol{\Psi}_{2} & \ddots & \vdots \\
\vdots & \ddots & \ddots & 0 \\
0 & \cdots & 0 & \boldsymbol{\Psi}_{\frac{M}{2}}
\end{array}\right], \quad \boldsymbol{\Psi}_{i}=\left[\begin{array}{cc}
\mathfrak{R e}\left(\bar{p}_{i}\right) & \mathfrak{I m}\left(\bar{p}_{i}\right) \\
-\mathfrak{I m}\left(\bar{p}_{i}\right) & \mathfrak{R e}\left(\bar{p}_{i}\right)
\end{array}\right], \quad i=\left(1,2, \ldots, \frac{M}{2}\right),
\end{aligned}
$$

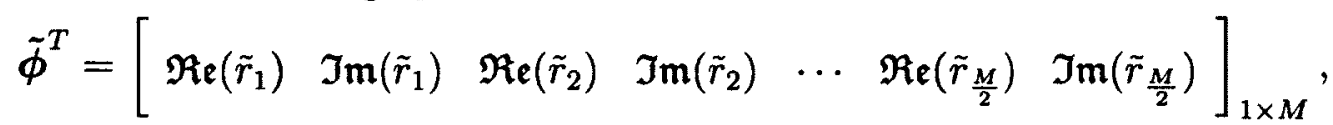

$$
\begin{aligned}
& \boldsymbol{g}^{T}=\left[\begin{array}{lllllll}
2 & 0 & 2 & 0 & \cdots & 2 & 0
\end{array}\right]_{1 \times M} .
\end{aligned}
$$

This modifies $\boldsymbol{\Psi}$ to become a real matrix with eigenvalues corresponding to the real and imaginary parts of the complex zeros in $\sigma(s)$.

\section{Common Pole Sets for Multiport Networks}

The vector fitting algorithm detailed in the previous sections was for a scalar function (single port network). However for multiport networks, a separate set of tabulated data is given for each element in the matrix transfer function. If the data for each matrix element is fit separately, then a large number of redundant poles could result, leading to inefficient transient simulations [39]. Therefore, in order to minimize the number of poles in the matrix-transfer functions, the following two propositions are used [78].

1) In general, the pole set corresponding to any individual admittance element is a subset of the union of all driving point admittances [79].

2) Generally, in a system with a large number of dominant poles, pole sets belonging to different driving point admittances contain mostly identical poles and 
only a very small percentage of poles differ among these sets.

A common pole set for a multiport network is obtained by performing the pole identification process described above on driving point admittances. The initial set of poles is found by first running the pole finding iterations on a single driving point admittance, resulting in faster convergence for the pole finding algorithm of the driving point functions [42].

It should be noted that while a common pole set can be found for admittance parameters, it has not been shown to be possible on scattering parameters.

\section{Relaxed Vector Fitting}

In some cases, the tabulated data may be noisy and conventional vector fitting may fail to converge due to the asymptotic requirement that the scaling function in (2.7) approaches 1 as $s \rightarrow \infty$. To improve convergence, a more general scalar function is introduced in the form [18]

$$
\sigma(s)=\sum_{m=1}^{M} \frac{\tilde{r}_{m}}{s-\bar{p}_{m}}+\tilde{r}_{0}
$$

where $\tilde{r}_{0}$ is real. To ensure this does not result in the null solution for the least squares problem in (2.11), the following additional constraint is used

$$
\mathfrak{R e}\left\{\sum_{n=1}^{N}\left(\sum_{m=1}^{M} \frac{\tilde{r}_{m}}{s_{n}-\bar{p}_{m}}+\tilde{r}_{0}\right)\right\}=N
$$

This modification is introduced by adding an extra row to the least squares problem in (2.11). 


\subsection{Macromodeling Issues: Stability, Causality,}

\section{and Passivity}

Rational function approximation based macromodeling techniques for networks characterized by tabulated data have been very successful in obtaining accurate transient simulations [14]-[22]. However, it is also important that macromodels preserve the system properties of the original network. For linear systems characterized by tabulated data in the frequency-domain, the macromodel must be stable, causal, and passive [80]. Since these issues can seriously affect the accuracy of transient SPICE simulations, they are discussed in detail below.

\subsubsection{Stability}

Recall that a macromodel is a set of differential equations characterizing the functionality of a physical device. For time-domain analysis of a system of differential equations, stability is important to guarantee that for any given bounded input, the output converges to some bounded equilibrium state [81]. An unstable system may produce an output of either continuously increasing magnitude (as shown in Fig. 2.1) or growing oscillations (as shown in Fig. 2.2). Neither of these results is an accurate representation of the behavior of the real, physical device. Consequently, all macromodels of stable devices must also be stable.

Numerous stability criteria for differential systems exist, and the selection of appropriate criteria depends on both the type of differential equations and their corresponding application [81]. This thesis is concerned with the asymptotic stability of macromodels in the form of linear time-invariant systems. For these systems, sta- 


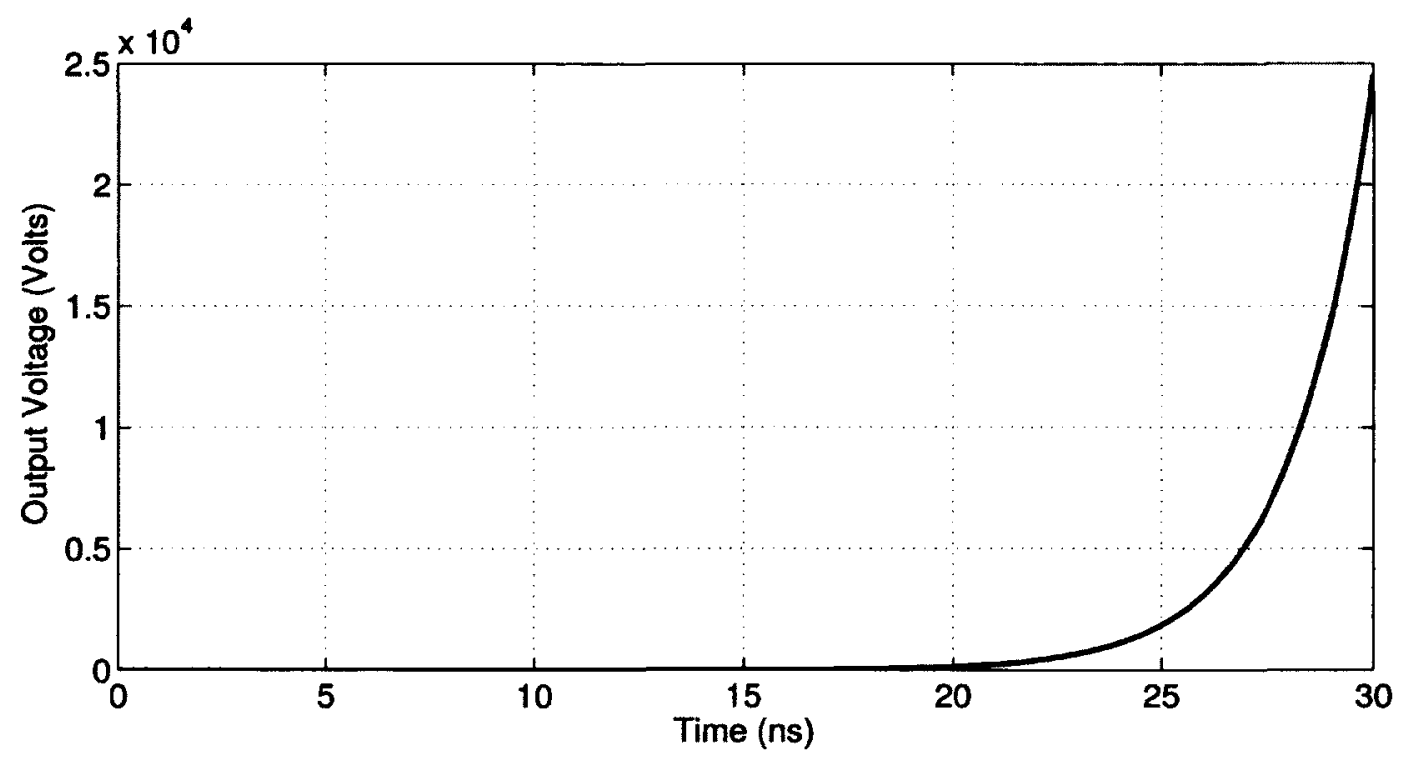

FIGURE 2.1: Unstable transient response monotonically and exponentially increasing in magnitude.

bility is more easily analyzed in the Laplace-domain. In particular, in this thesis a macromodel is considered asymptotically stable if and only if the poles of its transfer function lie in the left-half of the complex plane [82].

\subsubsection{Causality}

For any physical system, the corresponding output at any instant cannot depend on any future input, but only on past inputs. This natural physical property, known as causality, is stated more formally in the following definition [83].

Definition 2.1: $A$ system is causal if for any two arbitrary inputs $\boldsymbol{u}_{1}(t)$ and $\boldsymbol{u}_{2}(t)$ that satisfy

$$
\boldsymbol{u}_{1}(t)=\boldsymbol{u}_{2}(t), \quad \text { for } t<t_{0}
$$




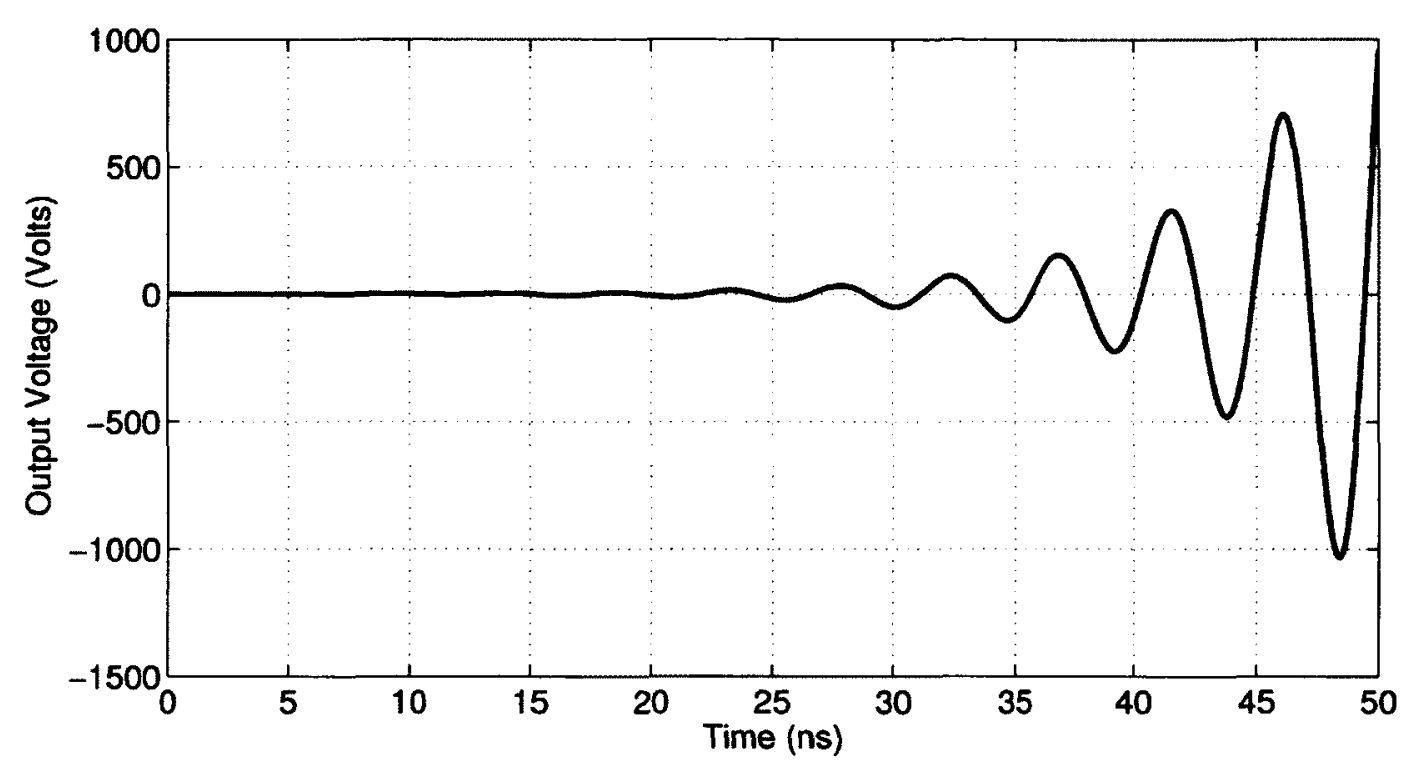

FIGURE 2.2: Unstable transient response with spurious oscillations.

the corresponding outputs $\boldsymbol{y}_{1}(t)$ and $\boldsymbol{y}_{2}(t)$ satisfy

$$
\boldsymbol{y}_{1}(t)=\boldsymbol{y}_{2}(t), \quad \text { for } t<t_{0}
$$

For the case of linear time-invariant systems, causality can be defined more simply in terms of its time-domain impulse response through the following theorem [83].

Theorem 2.1: A linear time-invariant system is causal if and only if its corresponding time-domain impulse response, $\boldsymbol{h}(t)$, satisfies

$$
\boldsymbol{h}(t)=\mathbf{0}, \quad \text { for } t<0 .
$$

To obtain accurate time-domain simulation results, it is important that the macromodel of a causal device is also causal [80]. 


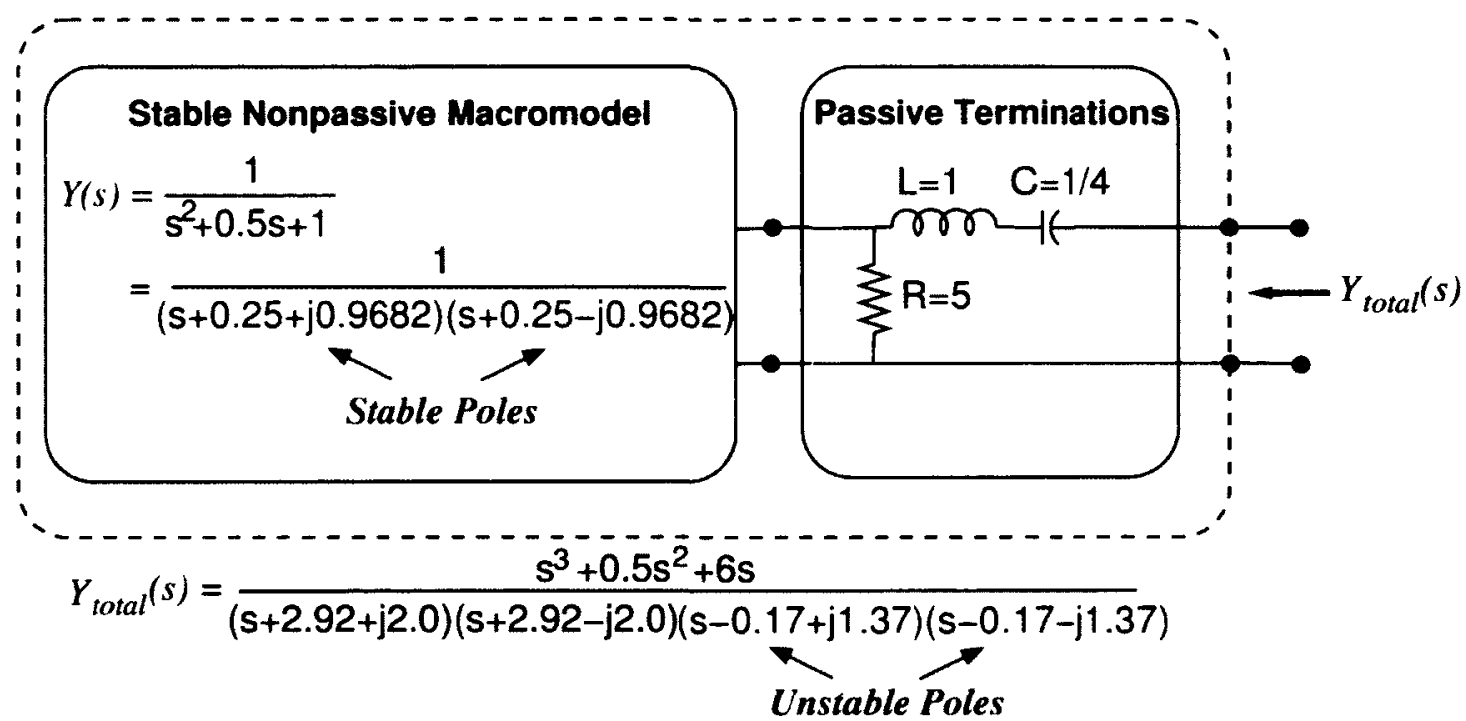

FIGURE 2.3: Illustration of a stable, but nonpassive macromodel connected with passive terminations. The overall network is unstable.

\subsubsection{Passivity}

A passive device is one that cannot generate more energy than it absorbs [84]. In this thesis, in addition to being stable and causal, the physical devices considered are also passive. When macromodeling tabulated data networks, direct fitting algorithms do not guarantee that the resulting macromodel is passive. Passivity is important because a stable, but nonpassive system can become unstable when connected to other passive devices [80].

In order to illustrate this concept, consider the single port network shown in Fig. 2.3. The poles of the stable, nonpassive macromodel are clearly in the left-half plane. When passive terminations are connected to this macromodel, the poles of the transfer function for the overall network, $\boldsymbol{Y}_{\text {total }}(s)$, now lie in the right-half plane. Thus, the complete system is unstable, even though each separate network is stable. The unstable time-domain response from the overall network is shown in Fig. 2.2. 
The above problem can be overcome by creating macromodels that are passive since all passive components connected to other passive components are (collectively) passive [85]. In addition, although a stable system is not necessarily passive, all linear passive systems are stable. For linear time-invariant systems it was also shown that, if the system is passive, then it is also causal [83], [84]. Consequently, to ensure a macromodel for a linear time-invariant network is stable, causal, and passive, it is sufficient to only verify and enforce passivity. For this purpose, a review of the mathematical conditions for passivity in the immitance (either admittance or impedance) and scattering domain is given in the following sections.

\section{Passivity of Immitance Domain Systems}

For immitance domain systems, passivity is defined in the time-domain as follows [83]:

Definition 2.2: An n-port network in the immitance domain is passive if

$$
\int_{-\infty}^{t} v^{T}(\eta) i(\eta) d \eta \geq 0
$$

for all $t . \boldsymbol{v}(t)$ and $\boldsymbol{i}(t)$ are the port voltages and currents, respectively.

Although the above definition provides a means to verify if a macromodel is passive, it is only practical for small systems where the integral may be solved analytically. To address this issue, the passivity of linear time-invariant systems is typically considered in the Laplace-domain. The following theorem provides the necessary and sufficient conditions for a linear $n$-port immitance matrix, $\boldsymbol{H}(s)$, to be passive in the Laplacedomain [86].

Theorem 2.2: $A$ system defined by an $n \times n$ immitance matrix $\boldsymbol{H}(s)$ is passive if and only if 
a) $\boldsymbol{H}(s)$ is analytic for all values of $s$ with $\mathfrak{R e}(s)>0$;

b) $\boldsymbol{H}\left(s^{*}\right)=\boldsymbol{H}^{*}(s)$, where the superscript ' $*$ ' is the complex conjugate operator;

c) $\left[\boldsymbol{H}^{H}(s)+\boldsymbol{H}(s)\right]$ is nonnegative definite for $\mathfrak{R e}(s)>0$. That is

$$
\boldsymbol{z}^{H}\left[\boldsymbol{H}^{H}(s)+\boldsymbol{H}(s)\right] \boldsymbol{z} \geq 0
$$

for $\mathfrak{R e}(s)>0$ and for any arbitrary vector $\boldsymbol{z} \in \mathbb{R}^{n}$. Here, the superscript ' $H$ ' represents the Hermitian conjugate operator.

A transfer function, $\boldsymbol{H}(s)$, that satisfies the above 3 conditions is referred to as positive real. For the purpose of macromodeling, conditions a) and b) are generally easy to verify and enforce. Condition $c$ ) is equivalent to requiring that the real part of the eigenvalues of $\left[\boldsymbol{H}^{H}(s)+\boldsymbol{H}(s)\right]$ are nonnegative for $\mathfrak{R e}(s)>0$. In general, ensuring condition $c$ ) is a challenging task.

\section{Passivity of Scattering Domain Systems}

In many cases, macromodels are generated in the scattering domain. For this purpose, time-domain passivity criteria for scattering domain networks are given as follows [83]:

Definition 2.3: An n-port network in the scattering domain is passive if

$$
\int_{-\infty}^{t}\left[\boldsymbol{a}^{T}(\eta) \boldsymbol{a}(\eta)-\boldsymbol{b}^{T}(\eta) \boldsymbol{b}(\eta)\right] d \eta \geq 0
$$

for all t. $\boldsymbol{a}(t)$ and $\boldsymbol{b}(t)$ are the incident and reflected power waves, respectively.

Equivalently, in the necessary and sufficient conditions for a scattering matrix to be passive in the Laplace-domain are given by the following theorem [86]. 
Theorem 2.3: A system defined by an $n \times n$ scattering matrix $\boldsymbol{S}(s)$ is passive if and only if

a) $\boldsymbol{S}(s)$ is analytic for all values of $s$ with $\mathfrak{R e}(s)>0$;

b) $\boldsymbol{S}\left(s^{*}\right)=\boldsymbol{S}^{*}(s)$;

c) $\left[\boldsymbol{I}-\boldsymbol{S}^{H}(s) \boldsymbol{S}(s)\right]$ is nonnegative definite for $\mathfrak{R e}(s)>0$. That is

$$
\boldsymbol{z}^{H}\left[I-S^{H}(s) S(s)\right] z \geq 0
$$

for $\mathfrak{R e}(s)>0$ and for any arbitrary vector $\boldsymbol{z} \in \mathbb{R}^{n}$.

A transfer function, $\boldsymbol{S}(s)$, that satisfies the above 3 conditions is said to be bounded real. Condition c) is equivalent to requiring that the 2-norm of the scattering parameters is less than or equal to 1 for $\mathfrak{R e}(s)>0$ (i.e. $\|S\| \leq 1$ for $\mathfrak{R e}(s)>0$ ). As in the case of the immitance parameters, ensuring condition c) is the primary challenge to constructing passive macromodels.

\subsection{Passive Rational Function based Macromodel- ing}

A major advantage of rational function based macromodeling techniques is that, passivity verification and compensation has been well addressed in the literature [36]-[49]. For passivity verification, prominent methodologies are based on solving full-size and half-size Hamiltonian matrices [35]-[41]. A primary advantage of Hamiltonian based passivity verification techniques is that, they accurately identify and 
quantify regions of passivity violation by solving a single algebraic eigenvalue problem [35], [39]-[41]. For passivity compensation, several different methodologies have been proposed [39]-[47]. The main idea behind these techniques is to iteratively perturb the residues or poles of the macromodel until it becomes passive.

The following sections outline the passive rational function based macromodeling techniques most relevant to this thesis. First, Hamiltonian based passivity verification techniques [41] are reviewed. Subsequently, passivity enforcement approaches based on first-order perturbation theory and linear matrix inequalities [42] are discussed. Details are provided in the scattering domain, however, similar techniques have also been developed in the admittance domain [39], [40].

\subsubsection{Passivity Verification}

Let the scattering equations of an $L$-port network be in the form $\boldsymbol{b}(s)=\boldsymbol{S}(s) \boldsymbol{a}(s)$, where $\boldsymbol{a}(s)$ and $\boldsymbol{b}(s)$ are the incident and reflected waves in the Laplace-domain, respectively. Also, let $\boldsymbol{S}(s)=\left[S_{i k}(s)\right], i, k \in\{1,2, \ldots, L\}$ represent the scattering matrix. Then each of the entries in the scattering matrix is defined as

$$
S_{i k}(s)=D_{0}^{(i, k)}+\sum_{n=1}^{N^{(i, k)}} \frac{R_{n}^{(i, k)}}{s-p_{n}^{(i, k)}}
$$

where for each $S_{i k}(s): N^{(i, k)}, R_{n}^{(i, k)}$, and $p_{n}^{(i, k)}$ are the total number of poles, the residues, and the poles, respectively. The equivalent time-domain state-space formu- 
lation is given by

$$
\begin{aligned}
\dot{\boldsymbol{x}}(t) & =\boldsymbol{A} \boldsymbol{x}(t)+\boldsymbol{B} \tilde{\boldsymbol{a}}(t) \\
\tilde{\boldsymbol{b}}(t) & =\boldsymbol{C x}(t)+\boldsymbol{D} \tilde{\boldsymbol{a}}(t)
\end{aligned}
$$

where $\boldsymbol{x}(t)$ represents the state variables, $\tilde{\boldsymbol{a}}(t)$ and $\tilde{\boldsymbol{b}}(t)$ are the time-domain representations of the incident and reflected waves $(\boldsymbol{a}(s)$ and $\boldsymbol{b}(s))$, respectively. $\boldsymbol{A} \in \mathbb{R}^{P \times P}$, $\boldsymbol{B} \in \mathbb{R}^{P \times L}, \boldsymbol{C} \in \mathbb{R}^{L \times P}, \boldsymbol{D} \in \mathbb{R}^{L \times L}$, and $P$ is the order of the approximation. The corresponding Laplace-domain transfer function is

$$
\boldsymbol{S}(s)=\boldsymbol{C}(s \boldsymbol{I}-\boldsymbol{A})^{-1} \boldsymbol{B}+\boldsymbol{D}
$$

where $I \in \mathbb{R}^{P \times P}$ is the identity matrix.

Recall from Section 2.3.3 that the necessary and sufficient conditions for a scattering matrix to be passive are given by Theorem 2.3. Conditions a) and b) are generally easy to verify by direct inspection. In the case of rational functions, they simply require the poles to be in the left-half plane and for the poles/zeros to be either real or come in complex conjugate pairs. For condition c) at $s=\infty$, it is easy to verify that $\|\boldsymbol{D}\|<1$. These conditions are also all easily ensured during the fitting process [15], [17], [42].

However, direct verification of the remainder of condition c) is a computationally intensive task. It requires checking that the 2-norm of $\boldsymbol{S}(s)$ is less than or equal to 1 for all values of $s$ in the right-half of the complex plane. In many cases passivity violations may be missed if the search grid is chosen too coarse. Since this is not practical, macromodel passivity verification algorithms focus on reducing this search 
region (for example, to just the imaginary axis), re-formulating passivity conditions as linear matrix inequalities, and re-formulating passivity verification as an algebraic eigenvalue problem. For this purpose, for the specific case of rational function based macromodels, the following theorem was introduced [86].

Theorem 2.4: The scattering matrix, $\boldsymbol{S}(s)$, described by rational functions is passive if and only if

a) $\boldsymbol{S}(s)$ is analytic for all values of $s$ with $\mathfrak{R e}(s)>0$;

b) $\boldsymbol{S}\left(s^{*}\right)=\boldsymbol{S}^{*}(s)$, where the superscript ' $*$ ' is the complex conjugate operator;

c) The norm of $\boldsymbol{S}(j \omega)$ is less than 1 for $\omega \in \mathbb{R}$. That is

$$
\boldsymbol{z}^{H}\left[\boldsymbol{I}-\boldsymbol{S}^{H}(j \omega) \boldsymbol{S}(j \omega)\right] \boldsymbol{z} \geq 0
$$

for all real values of $\omega$ and for any arbitrary vector $\boldsymbol{z}$. Here, the superscript ' $H$ ' represents the Hermitian conjugate operator.

The above theorem reduces condition c) in Theorem 2.3, from the entire right-half plane to just the imaginary axis. However, direct verification of $\|\boldsymbol{S}(j \omega)\| \leq 1$ over all values of $\omega \in \mathbb{R}$ is still a challenging task, and requires a fine frequency-sweep along the entire imaginary axis. To address this issue, an algebraic test was introduced via the following theorem [42], [87].

Theorem 2.5: The state-space system in (2.38) with $\boldsymbol{D}^{T} \boldsymbol{D}<\boldsymbol{I}$ is bounded real if and only if the following Hamiltonian matrix, $\boldsymbol{M}$, has no pure imaginary eigenvalues

$$
\boldsymbol{M}=\left[\begin{array}{cc}
\boldsymbol{A}+\boldsymbol{B}\left(\boldsymbol{I}-\boldsymbol{D}^{T} \boldsymbol{D}\right)^{-1} \boldsymbol{D}^{T} \boldsymbol{C} & \boldsymbol{B}\left(\boldsymbol{I}-\boldsymbol{D}^{T} \boldsymbol{D}\right)^{-1} \boldsymbol{B}^{T} \\
-\boldsymbol{C}^{T}\left(\boldsymbol{I}-\boldsymbol{D} \boldsymbol{D}^{T}\right)^{-1} \boldsymbol{C} & -\boldsymbol{A}^{T}-\boldsymbol{C}^{T} \boldsymbol{D}\left(\boldsymbol{I}-\boldsymbol{D}^{T} \boldsymbol{D}\right)^{-1} \boldsymbol{B}^{T}
\end{array}\right]
$$


Theorem 2.5 can be used to verify if a rational function based macromodel is bounded real and hence, passive. A primary advantage of using this theorem is that, it is independent of frequency and only a single eigenvalue calculation needs to be performed on the Hamiltonian $\boldsymbol{M}$. If there are no pure imaginary eigenvalues, then the model is passive. However, if the model is found to be nonpassive, then Theorem 2.5 does not provide a means to identify and quantify the regions of passivity violation. In this case, a frequency-sweep is still required. To address this issue, the following theorem was introduced [35], [42], [88].

Theorem 2.6: $\boldsymbol{S}\left(j \omega_{0}\right)$ has a maximum singular value equal to one (or equivalently, a norm equal to one) if and only if $j \omega_{0}$ is an imaginary eigenvalue of $\boldsymbol{M}$, provided $\boldsymbol{A}$ has no imaginary eigenvalues and $\boldsymbol{D}$ does not have a singular value equal to one.

From Theorem 2.6, it is clear that the pure imaginary eigenvalues of $\boldsymbol{M}$ correspond to the frequencies at which the norm of $\boldsymbol{S}(s)$ becomes equal to 1 . For a nonpassive macromodel, this means that the pure imaginary eigenvalues, $\omega_{0}$, correspond to crossing points where the singular values become equal to 1 . Using this information and the slopes of the function $\boldsymbol{S}^{H}(j \omega) \boldsymbol{S}(j \omega)$, the regions of passivity violation can be found with the technique summarized in Algorithm 1 [42].

Once the regions of passivity violation are obtained, the location of maximum violation in each region is identified. For this purpose, a frequency-sweep of $\|\boldsymbol{S}(j \omega)\|$ is performed over each region of passivity violation $\left(\omega_{l}, \omega_{u}\right)$. The frequency at which the maximum norm occurs, $\omega_{\max }$, is taken as the maximum violation in each region. It is to be noted that only an estimate of the maximum violation point is necessary for the passivity compensation process. 
Algorithm 1 Pseudocode for determining passivity violation regions

Step 1: Calculate the eigenvalues of the Hamiltonian matrix $\boldsymbol{M}$ in (2.41). If there are no pure imaginary eigenvalues, then the model is passive and we are done. Otherwise, proceed to step 2.

Step 2: Consider the pure imaginary eigenvalues with positive coefficient given by the set $\left(\omega_{1}<\omega_{2}<\ldots<\omega_{T}\right)$. Then, this set of coefficients corresponds to the frequencies at which the singular values of $\boldsymbol{S}^{H}(j \omega) \boldsymbol{S}(j \omega)$ become equal to one. Evaluate the slopes of $\boldsymbol{S}^{H}(j \omega) \boldsymbol{S}(j \omega)$ at each of these frequency points.

Step 3: Let $\omega_{S}=\omega_{T}$. Since $\|\boldsymbol{D}\|<1$ the model is passive at $\omega=\infty$ and so $\omega_{S}$ corresponds to the boundary point of the first passivity violation region and its corresponding slope is negative.

Step 4: Count the number of positive and negative slopes starting with $\omega_{S}$ and working backwards through the set $\left(\omega_{1}<\omega_{2}<\ldots<\omega_{T}\right)$. When the number of positive and negative slopes become equal, then this point corresponds to the other boundary point of the passivity violation region. Let $\omega_{k}$ be this point.

Step 5: Reset the slope counts to zero and let $\omega_{S}=\omega_{k-1}$. Repeat Steps 4 and 5 until all the crossing points have been used up.

\subsubsection{Passivity Compensation}

In this section, two passivity compensation approaches based on perturbing the residues of the rational functions are presented [42]. First, a method that uses firstorder perturbation theory is reviewed. Second, an algorithm based on solving linear matrix inequalities is described.

\section{First-Order Perturbation Theory Approach}

In this approach, the residues are iteratively perturbed such that at each iteration, the eigenvalues of $\left[\boldsymbol{I}-\boldsymbol{S}^{H}(j \omega) \boldsymbol{S}(j \omega)\right]$ become less negative. The compensation process is completed when the eigenvalues are all positive and hence, the macromodel is passive. An illustration of the perturbation process is given in Fig. 2.4. Details of the algorithm are as follows.

For simplicity, assume there is only a single passivity violation in the macromodel 


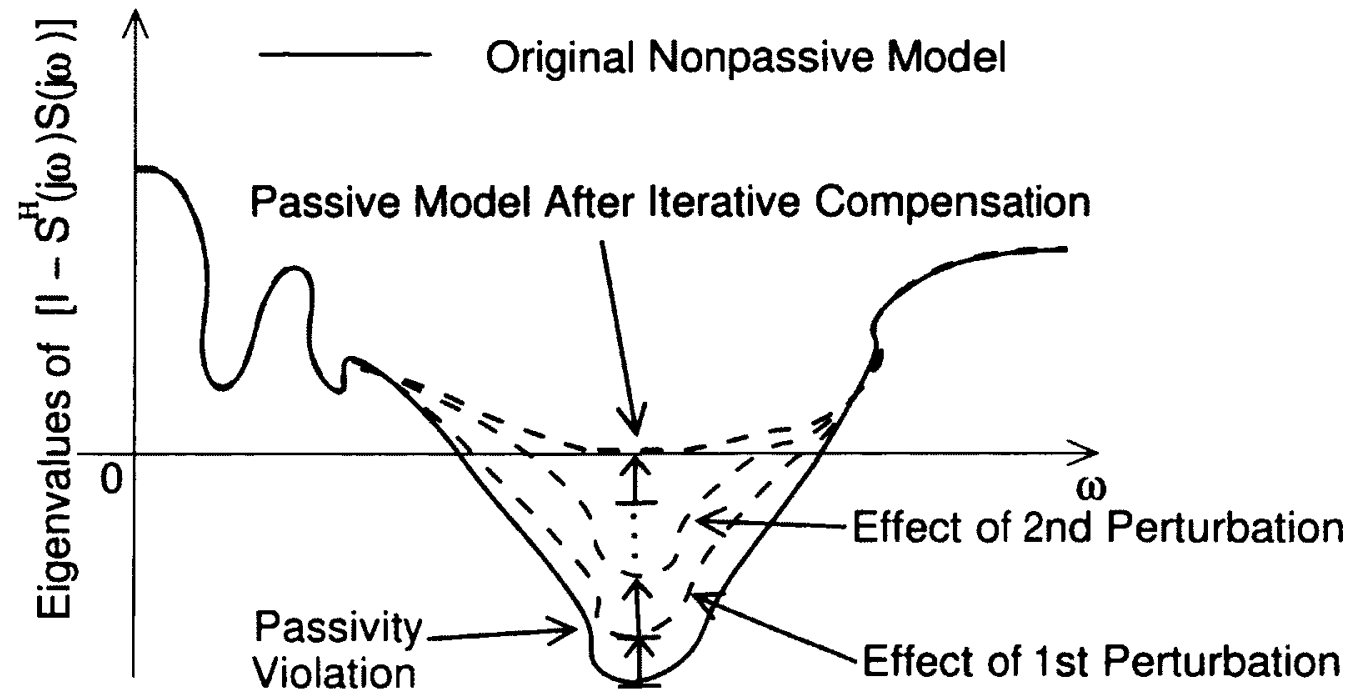

FIGURE 2.4: Illustration of iterative perturbation process.

between $\left(\omega_{l}, \omega_{h}\right)$ and the maximum violation in the region occurs at $\omega_{\max }$. Recall that $C$ in (2.39) corresponds to the residues of the rational function [5]. Then, let $\Delta C$ be the desired perturbation of the residues and let

$$
\tilde{C}=C+\Delta C
$$

be the residues of the perturbed model. The scattering parameters of the perturbed macromodel are then given by

$$
\tilde{\boldsymbol{S}}(j \omega)=\boldsymbol{S}(j \omega)+\boldsymbol{\Delta} \boldsymbol{S}(j \omega)
$$

where

$$
\boldsymbol{S}(j \omega)=\boldsymbol{C}(j \omega \boldsymbol{I}-\boldsymbol{A})^{-1} \boldsymbol{B}+\boldsymbol{D}, \quad \boldsymbol{\Delta} \boldsymbol{S}(j \omega)=\boldsymbol{\Delta} \boldsymbol{C}(j \omega \boldsymbol{I}-\boldsymbol{A})^{-1} \boldsymbol{B} .
$$


It is desired to perturb the eigenvalues of the matrix $\left[\boldsymbol{I}-\boldsymbol{S}^{H}(j \omega) \boldsymbol{S}(j \omega)\right]$ at $\omega=$ $\omega_{\max }$ such that the added perturbation $\boldsymbol{\Delta} \boldsymbol{S}$ makes the eigenvalues less negative (for simplicity, the $(j \omega)$ is dropped in the following derivations). For this purpose, recall from first-order perturbation theory [89] that if a given matrix $\boldsymbol{T}$ is perturbed by $\Delta T$, then each eigenvalue is perturbed by

$$
\frac{y^{T} \Delta \boldsymbol{T} x}{y^{T} \boldsymbol{x}}
$$

where $\boldsymbol{y}$ and $\boldsymbol{x}$ are the corresponding left and right eigenvectors of $\boldsymbol{T}$, respectively. Now compare the bounded real condition for the perturbed model given by

$$
\boldsymbol{I}-\tilde{\boldsymbol{S}}^{H} \tilde{\boldsymbol{S}}=\boldsymbol{I}-\boldsymbol{S}^{H} \boldsymbol{S}-\boldsymbol{S}^{H} \Delta \boldsymbol{S}-\Delta \boldsymbol{S}^{H} \boldsymbol{S}-\Delta \boldsymbol{S}^{H} \Delta \boldsymbol{S} \geq 0
$$

with the bounded real condition for the original model

$$
\boldsymbol{I}-\boldsymbol{S}^{H} \boldsymbol{S} \geq \mathbf{0}
$$

It is clear that the perturbation introduced in the original model is given by

$$
-\boldsymbol{S}^{H} \boldsymbol{\Delta} \boldsymbol{S}-\boldsymbol{\Delta} \boldsymbol{S}^{H} \boldsymbol{S}-\boldsymbol{\Delta} \boldsymbol{S}^{H} \boldsymbol{\Delta} \boldsymbol{S}
$$

Neglecting the second order term $\Delta \boldsymbol{S}^{H} \boldsymbol{\Delta} \boldsymbol{S}$ and using (2.45), the contribution to the negative eigenvalue from the perturbation is given by

$$
\Delta \lambda=\frac{v^{T}\left(-S^{H} \Delta S-\Delta S^{H} S\right) u}{v^{T} u}
$$


where $\boldsymbol{v}$ and $\boldsymbol{u}$ are the left and right eigenvectors of $\left[\boldsymbol{I}-\boldsymbol{S}^{H}\left(j \omega_{\max }\right) \boldsymbol{S}\left(j \omega_{\max }\right)\right]$. The goal of the perturbation process is for $\left[-S^{H} \boldsymbol{\Delta S}-\Delta \boldsymbol{S}^{H} \boldsymbol{S}\right]$ to cancel $-\Delta \lambda$ from the negative eigenvalue of $\left[\boldsymbol{I}-\boldsymbol{S}^{H} \boldsymbol{S}\right]$ for the original system.

Using the fact that $\boldsymbol{v}^{T} \boldsymbol{u}=1$ and substituting (2.44) for $\boldsymbol{S}$ and $\Delta \boldsymbol{S}$ into (2.49) yields

$$
\begin{aligned}
\Delta \lambda= & \boldsymbol{v}^{T}\left[\boldsymbol{D}^{T} \Delta \boldsymbol{C}(s \boldsymbol{I}-\boldsymbol{A})^{-1} \boldsymbol{B}+\boldsymbol{B}^{T}\left(s^{H} \boldsymbol{I}-\boldsymbol{A}^{T}\right)^{-1} C^{T} \Delta C(s \boldsymbol{I}-\boldsymbol{A})^{-1} \boldsymbol{B}+\right. \\
& \left.\boldsymbol{B}^{T}\left(s^{H} \boldsymbol{I}-\boldsymbol{A}^{T}\right)^{-1} \Delta \boldsymbol{C}^{T} \boldsymbol{C}(s \boldsymbol{I}-\boldsymbol{A})^{-1} \boldsymbol{B}+\boldsymbol{B}^{T}\left(s^{H} \boldsymbol{I}-\boldsymbol{A}^{T}\right)^{-1} \Delta \boldsymbol{C}^{T} \boldsymbol{D}\right] \boldsymbol{u}
\end{aligned}
$$

where $s=j \omega$. Next, (2.50) is rearranged using the property of Kronecker products $[90]$ as

$$
\begin{array}{r}
\Delta \lambda=\left[\left((s \boldsymbol{I}-\boldsymbol{A})^{-1} \boldsymbol{B} \boldsymbol{u}\right)^{T} \otimes \boldsymbol{v}^{T} \boldsymbol{D}^{T}+\left((s \boldsymbol{I}-\boldsymbol{A})^{-1} \boldsymbol{B} \boldsymbol{u}\right)^{T} \otimes \boldsymbol{B}^{T}\left(s^{H} \boldsymbol{I}-\boldsymbol{A}^{T}\right)^{-1} \boldsymbol{C}^{T}+\right. \\
\left.\quad\left((s \boldsymbol{I}-\boldsymbol{A})^{-1} \boldsymbol{B} \boldsymbol{v}\right)^{T} \otimes \boldsymbol{u}^{T} \boldsymbol{B}^{T}\left(s^{H} \boldsymbol{I}-\boldsymbol{A}^{T}\right)^{-1} \boldsymbol{C}^{T}+\left((s \boldsymbol{I}-\boldsymbol{A})^{-1} \boldsymbol{B} \boldsymbol{v}\right)^{T} \otimes \boldsymbol{u}^{T} \boldsymbol{D}^{T}\right] \boldsymbol{x}
\end{array}
$$

where $\otimes$ represents the Kronecker product operator and $x=v e c(\Delta C)$ corresponds to the rows of $\Delta C$ stacked in vector format. This can be written as a least squares problem in the form

$$
\boldsymbol{W} \boldsymbol{x}=\Delta \lambda
$$

where $\boldsymbol{x}=\operatorname{vec}(\Delta \boldsymbol{C})$ are the desired residue perturbations of the matrix $\Delta \boldsymbol{C}$ and $\boldsymbol{W}$ is the remaining term between the square brackets in (2.51). For multiple regions of passivity violation, (2.52) is solved at each of the frequencies of maximum violation.

With the perturbation $\Delta C$ added to the original macromodel, it is inevitable that $\boldsymbol{\Delta} \boldsymbol{S}(j \omega)$ introduces error in the overall transfer function. In order to minimize this 
error, constraints are imposed during the perturbation process as follows.

First, note that the $L_{2}$-norm of the response can be written as [91]

$$
\|\Delta S(j \omega)\|_{2}^{2}=\int_{-\infty}^{\infty}\|\Delta S(j \omega)\|_{F}^{2} d \omega=\operatorname{trace}\left(\Delta C P \Delta C^{T}\right)
$$

where $\|\cdot\|_{2}$ and $\|\cdot\|_{F}$ are the $L_{2}$-norm and Frobenius norm of the argument. The matrix $\boldsymbol{P}$ is the controllability Grammian obtained from solving the Lyapunov equation

$$
A P+P A^{H}+B B^{H}=0
$$

Since (2.54) is independent of $\boldsymbol{C}, \boldsymbol{P}$ is calculated once at the beginning of the passivity compensation process. Incorporating error control in the compensation procedure, the problem to solve is

$$
\begin{gathered}
\min _{\Delta \boldsymbol{C}}\left(\operatorname{trace}\left(\Delta \boldsymbol{C P} \boldsymbol{\Delta} \boldsymbol{C}^{T}\right)\right) \quad \text { subject to } \\
\boldsymbol{W} \boldsymbol{x}=\Delta \lambda
\end{gathered}
$$

where $\boldsymbol{W}$ and $\boldsymbol{x}$ are defined in (2.52).

\section{Linear Matrix Inequality Approach}

In this section, an approach to passivity compensation based on linear matrix inequalities is reviewed [41]. The goal of passivity compensation is to obtain a perturbed model $\tilde{\boldsymbol{S}}(s)$ that satisfies passivity condition $c$ ) of Theorem 2.4 and is bounded real. Using condition c) and (2.46) (neglecting the second order term), note that the per- 
turbed model needs to satisfy the linear matrix inequality given by

$$
\boldsymbol{I}-\boldsymbol{S}^{H} \boldsymbol{S}-\boldsymbol{S}^{H} \Delta \boldsymbol{S}-\Delta \boldsymbol{S}^{H} \boldsymbol{S} \geq \mathbf{0}
$$

Substituting in (2.44) and using $\boldsymbol{F}=(j \omega \boldsymbol{I}-\boldsymbol{A})^{-1} \boldsymbol{B}$ gives

$$
\boldsymbol{I}-\boldsymbol{S}^{H} \boldsymbol{S}-\boldsymbol{S}^{H} \Delta \boldsymbol{C F}-\Delta \boldsymbol{C}^{H} \boldsymbol{F}^{H} \boldsymbol{S} \geq \mathbf{0}
$$

This problem is linear in terms of the unknown matrix $\Delta C$ and can be solved using interior point methods [87].

In many cases, all the terms in (2.57) are not necessarily pure real matrices (this is typically the case for unsymmetrical macromodels). In practice, this can be a problem since many linear matrix inequality solvers cannot directly handle complex functions. For this purpose, the following lemma is used.

Lemma 2.7: A complex Hermitian matrix, $\Upsilon$, is positive semi-definite if and only if

$$
\left[\begin{array}{cc}
\mathfrak{R e}(\mathbf{\Upsilon}) & \operatorname{Im}(\mathbf{\Upsilon}) \\
-\mathfrak{I m}(\boldsymbol{\Upsilon}) & \mathfrak{R e}(\mathbf{\Upsilon})
\end{array}\right] \geq \mathbf{0}
$$

Proof of Lemma 2.7: To prove necessity, the process given in [42] (pg. 84) for positive definiteness is followed with ' $>$ ' replaced with ' $\geq$ '. Sufficiency can be proven working in the reverse manner. 
Using Lemma 2.7 and defining $\boldsymbol{S}=\boldsymbol{S}_{\boldsymbol{R}}+j \boldsymbol{S}_{I}$ and $\boldsymbol{F}=\boldsymbol{F}_{R}+j \boldsymbol{F}_{I},(2.57)$ becomes

$$
\begin{aligned}
{\left[\begin{array}{cc}
\boldsymbol{S}_{R} & \boldsymbol{S}_{I} \\
-\boldsymbol{S}_{I} & \boldsymbol{S}_{R}
\end{array}\right]^{T} } & {\left[\begin{array}{cc}
\boldsymbol{S}_{R} & \boldsymbol{S}_{I} \\
-\boldsymbol{S}_{I} & \boldsymbol{S}_{R}
\end{array}\right]+\left[\begin{array}{cc}
\boldsymbol{S}_{R} & \boldsymbol{S}_{I} \\
-\boldsymbol{S}_{I} & \boldsymbol{S}_{R}
\end{array}\right]^{T}\left[\begin{array}{cc}
\Delta C & 0 \\
0 & \Delta C
\end{array}\right]\left[\begin{array}{cc}
\boldsymbol{F}_{R} & \boldsymbol{F}_{I} \\
-\boldsymbol{F}_{I} & \boldsymbol{F}_{R}
\end{array}\right] } \\
& +\left[\begin{array}{cc}
\Delta C & 0 \\
0 & \Delta C
\end{array}\right]^{T}\left[\begin{array}{cc}
\boldsymbol{F}_{R} & \boldsymbol{F}_{I} \\
-\boldsymbol{F}_{I} & \boldsymbol{F}_{R}
\end{array}\right]^{T}\left[\begin{array}{cc}
\boldsymbol{S}_{R} & \boldsymbol{S}_{I} \\
-S_{I} & \boldsymbol{S}_{R}
\end{array}\right] \leq \boldsymbol{I} .
\end{aligned}
$$

To minimize the error introduced during this perturbation process, the techniques developed in the previous section can be applied here as well. Combining (2.53) with (2.59), the passivity compensation process is to iteratively solve

$$
\min _{\Delta C}\left(\operatorname{trace}\left(\Delta \boldsymbol{C P} \Delta \boldsymbol{C}^{T}\right)\right) \quad \text { subject to }(2.59)
$$

until the macromodel becomes passive. 


\section{CHAPTER 3}

\section{Review of Macromodeling}

\section{Networks with Time-Delays}

The previous chapter provided a review of passive macromodeling techniques based on rational functions. However, rational function based approaches are not always optimum for tabulated data networks with time-delays. To address this, Delayed Rational Function (DRF) macromodeling techniques were developed for networks with time-delays characterized by tabulated data. This chapter provides an overview DRF macromodeling techniques for tabulated data. The chapter is organized as follows. Section 3.1 provides a review of issues that arise from applying rational macromodeling techniques to networks with time-delays. Section 3.2 reviews DRF macromodeling for tabulated data networks. Next, Section 3.3 reviews DRF fitting using Hilbert transform based delay estimation [31]-[34]. It is followed by Section 3.4, that reviews the Gabor transform based DRF macromodeling technique [28]. Section 3.5 presents the delayed vector fitting algorithm for DRF macromodeling [29], [30]. 


\subsection{Long Delay Networks: Macromodeling Issues}

As discussed in the Chapter 2, passive macromodeling based on rational functions has become a prominent tool for integrating tabulated data networks into standard SPICE simulators. However, for tabulated networks with long delays, there are several major drawbacks with rational function approximations.

1) Long delays require high-order rational approximations.

2) Rational models produce an unnatural ringing in the time-domain response.

3) Rational models produce unnatural rise time degradation and in turn, inaccurate timing analysis.

Further details are given in the following sections.

\subsubsection{High-Order Rational Approximations}

Delays are characterized in the frequency-domain by the exponential function $e^{-s \tau}$, where $\tau$ is the delay in the network. However, conventional macromodeling techniques [17]-[22] approximate this exponential function using rational functions in the form

$$
H(s)=\sum_{n=1}^{N} \frac{r_{n}}{s-p_{n}}+d
$$

where $r_{n}$ and $p_{n}$ are real or come in complex conjugate pairs representing the residues and poles, respectively. The value $d$ is real and $N$ is the number of poles. The exponential function has an infinite number of poles and zeros, while rational approximations contain only a finite number of each. Therefore, for high frequencies and/or long delays, an accurate rational function approximation of a single delay 


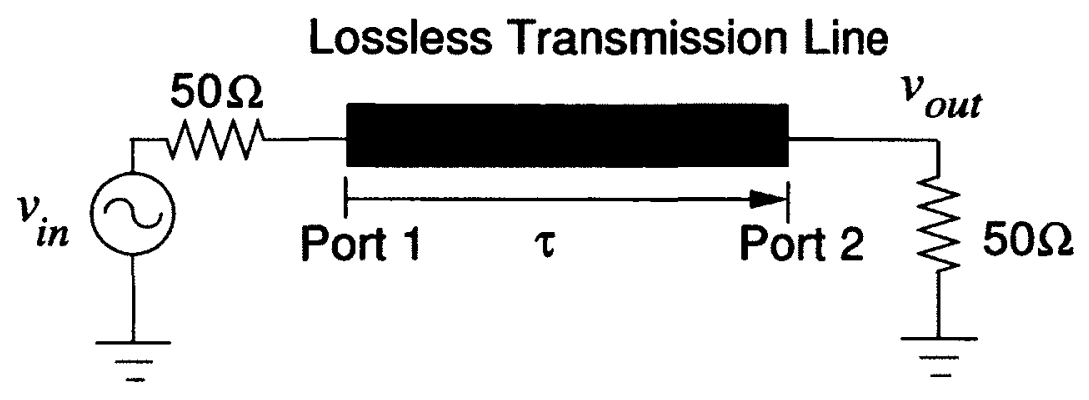

FIGURE 3.1: Two-port lossless transmission line network.

element can require hundreds of poles. Consequently, the corresponding equivalent circuit will require a large number of circuit elements and yield slow transient analysis. Furthermore, an unnatural ringing effect will be observed in the time-domain response.

\subsubsection{Ringing in the Response}

Consider an ideal, lossless two-port transmission line network shown in Fig. 3.1. A signal applied at port 1 takes a finite amount of time, $\tau$, to travel to port 2 . Then, there is no output response at port 2 until time $\tau$ (i.e. the voltage and current values are both 0 until time $\tau$ ). This phenomenon is illustrated by the flat portion of the response in Fig. 3.2, where here $\tau=8$ ns.

Assuming the line is perfectly matched and there are no reflections at port 2 , the output response at port 2 can be described mathematically as

$$
y(t)=\int_{0}^{t} h_{12}(t-x) v(x) d x=\int_{0}^{t} \delta(t-x-\tau) v(x) d x=v(t-\tau) u(t-\tau)
$$

Here, $h_{12}(t)=\delta(t-\tau)$ represents the time-domain impulse response at port 2 due to an input at port 1 and $v(t)$ is the input signal at port 1. $u(t)$ is the unit step function 


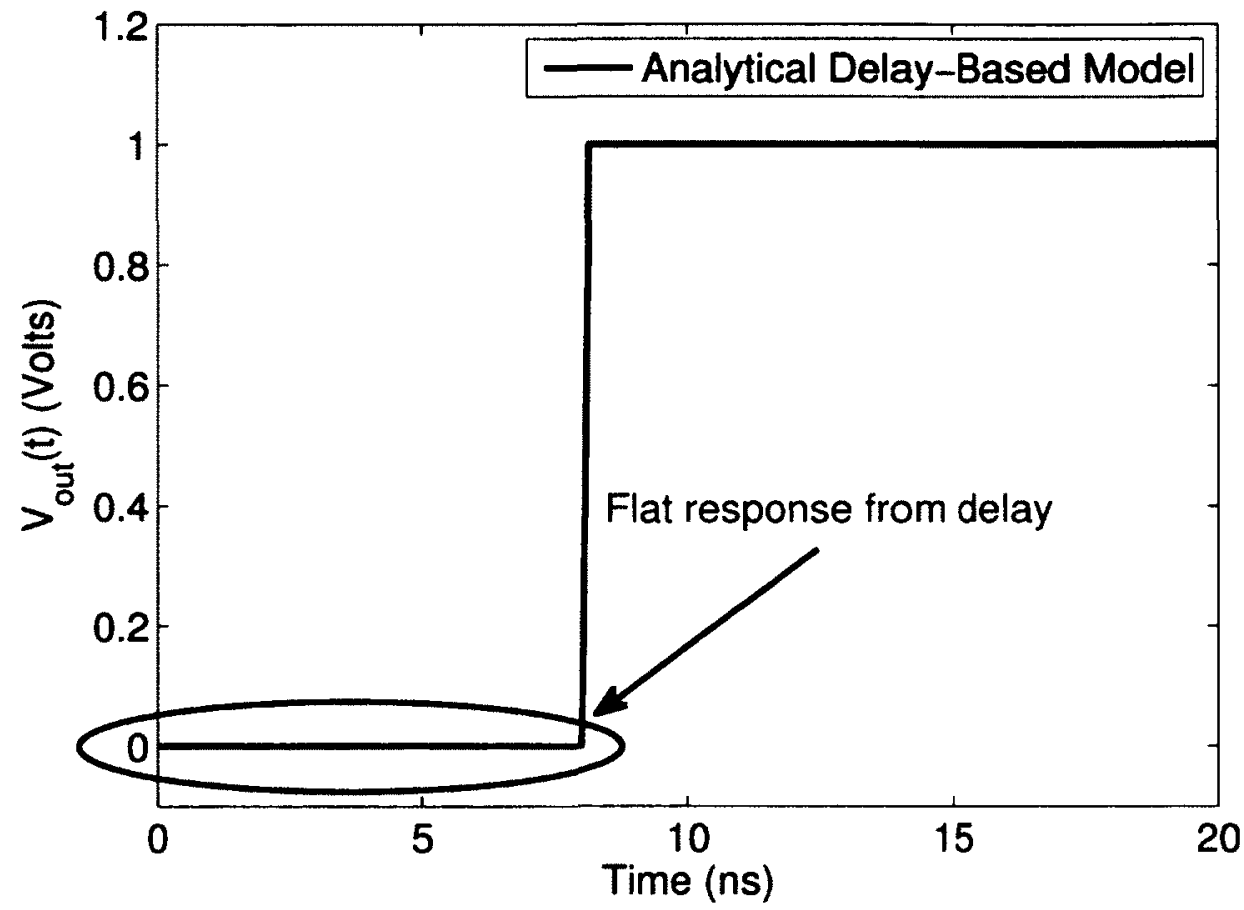

FIGURE 3.2: Illustration of port-to-port delay from a lossless transmission line.

defined by

$$
u(t)= \begin{cases}1 & \text { for } t \geq \tau \\ 0 & \text { for } t<\tau\end{cases}
$$

In the Laplace-domain, the transfer function for the response at port 2 due to an input at port 1 is given by

$$
H_{12}(s)=\mathfrak{L}\left(h_{12}(t)\right)=\mathfrak{L}(\delta(t-\tau))=e^{-s \tau}
$$

where $s$ is the independent variable of the Laplace-domain. Rational macromodeling 
techniques approximate the transfer function using a rational function such that

$$
H_{12}(s)=e^{-s \tau} \approx \sum_{n=1}^{N} \frac{r_{n}}{s-p_{n}}+d
$$

In the time-domain, this approximation of the impulse response, $\hat{h}_{12}(t)$, can be written as

$$
\hat{h}_{12}(t)=\sum_{n=1}^{N} r_{n} e^{t p_{n}}+d \delta(t) .
$$

Then, using (3.6) with (3.2), the approximate time-domain output response for the model, $\hat{y}(t)$, becomes

$$
\begin{aligned}
\hat{y}(t) & =\sum_{n=1}^{N}\left(r_{n} \int_{0}^{t} e^{(t-x) p_{n}} v(x) d x\right)+d \int_{0}^{t} \delta(t-x) v(x) d x \\
& =\sum_{n=1}^{N}\left(r_{n} \int_{0}^{t} e^{t p_{n}} v(x) d x\right)+d v(t)-d v(0) .
\end{aligned}
$$

It is clear that in general, the output from the rational model is nonzero for time $t$, $0<t<\tau$. Specifically, let the input be a unit step function (i.e. $v(t)=u(t))$. Then (3.7) becomes

$$
\hat{y}(t)=\sum_{n=1}^{N}\left(r_{n} / p_{n}\right)\left(e^{t p_{n}}-1\right) .
$$

The flat or zero portion of the response is now approximated as a sum of exponential terms. Consequently, there is a non-physical nonzero response for $0<t<\tau$, which gives the ringing effect in the transient response for rational function based macromodels. This effect becomes more pronounced as the delays become longer and the order of the rational function approximations increase.

To illustrate the ringing in the response, consider the lossless transmission line 
network in Fig. 3.1. The transfer function for the transmission line was approximated with a set of rational functions. Using the vector fitting algorithm [17], it required 80 poles to accurately approximate the tabulated data up to $2.5 \mathrm{GHz}$. The model was excited using a $2 \mathrm{~V}$ step function with a rise time of $0.15 \mathrm{~ns}$. The time-domain response using the rational function model is shown in Fig. 3.3. As seen, there is a nonzero ringing in the response between 0 and $8 \mathrm{~ns}$ while the analytical delay-based lossless transmission line model produces a zero output.

The error from this ringing can actually be seen throughout the time-domain response, and is also prominent around rising/falling clock edges. Fig. 3.4 shows the ringing effect after the rising edge of the unit step function reaches port 2 . This ringing is a significant source of error from macromodels based on rational functions.

\subsubsection{Inaccurate Timing Analysis}

Consider the ideal lossless transmission line from the previous section along with its corresponding rational and analytical macromodels. Fig. 3.5 gives an expanded view of the rising edge of the clock in response to a 0.15 ns rise time input signal. The rational macromodel shows the switching response begins at 7.94 ns while the analytical delay-based model begins switching at $7.99 \mathrm{~ns}$, a discrepancy of $0.05 \mathrm{~ns}$. In relation to the delay of $8 \mathrm{~ns}$ this error may seem acceptable, however, the effect of this error in relation to the rise time can be considerable.

The rise times (10\% to $90 \%$ of total rising edge) for the rational and analytical macromodels were $0.194 \mathrm{~ns}$ and $0.121 \mathrm{~ns}$, respectively. A discrepancy of $0.073 \mathrm{~ns}$ or equivalently, an error of $60 \%$ from the rational macromodel. This unnatural rise time degradation leads to overly conservative estimates for the maximum and minimum 


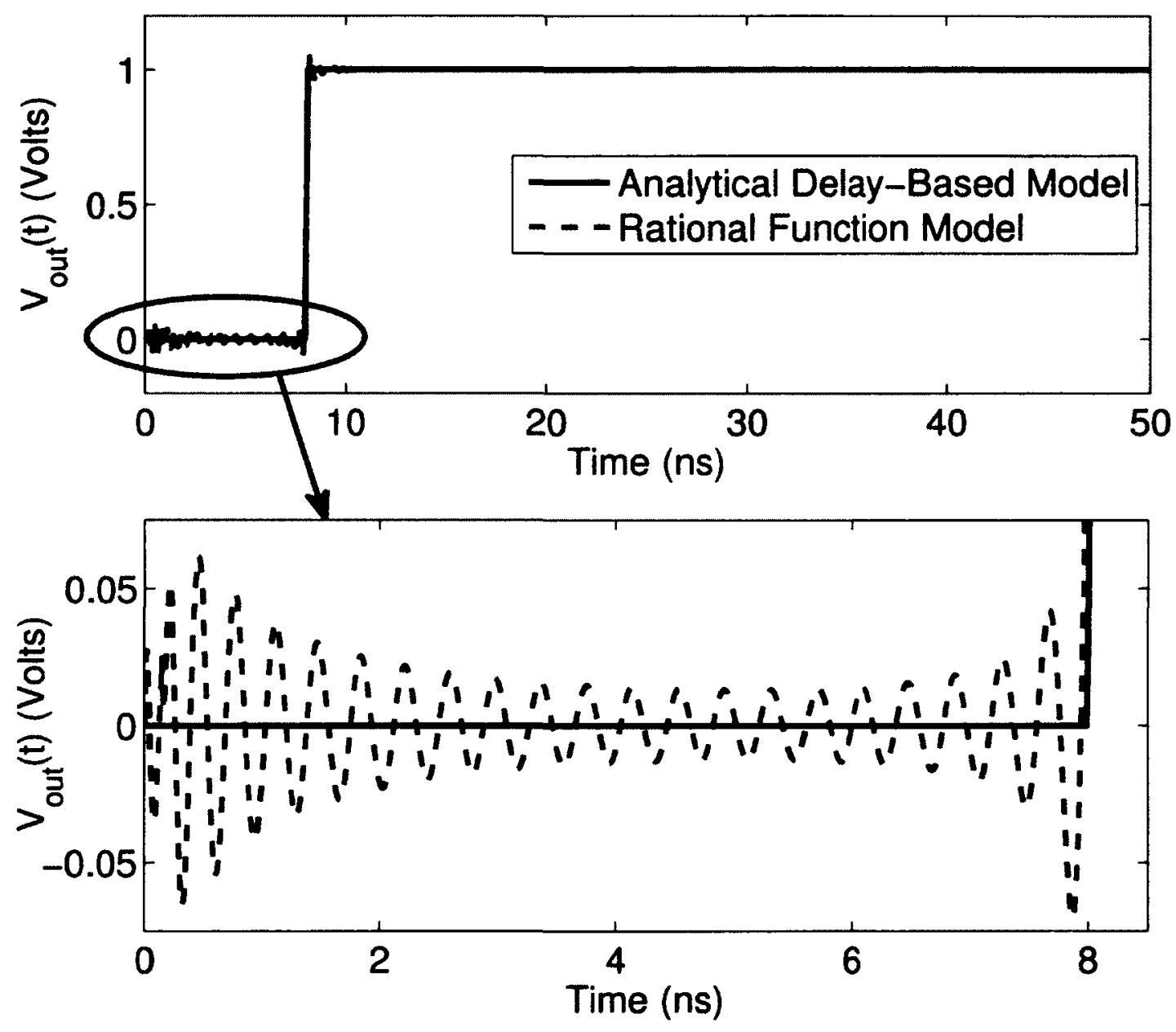

FIGURE 3.3: Illustration of spurious ringing effect from approximating an exponential delay function using a rational function. 


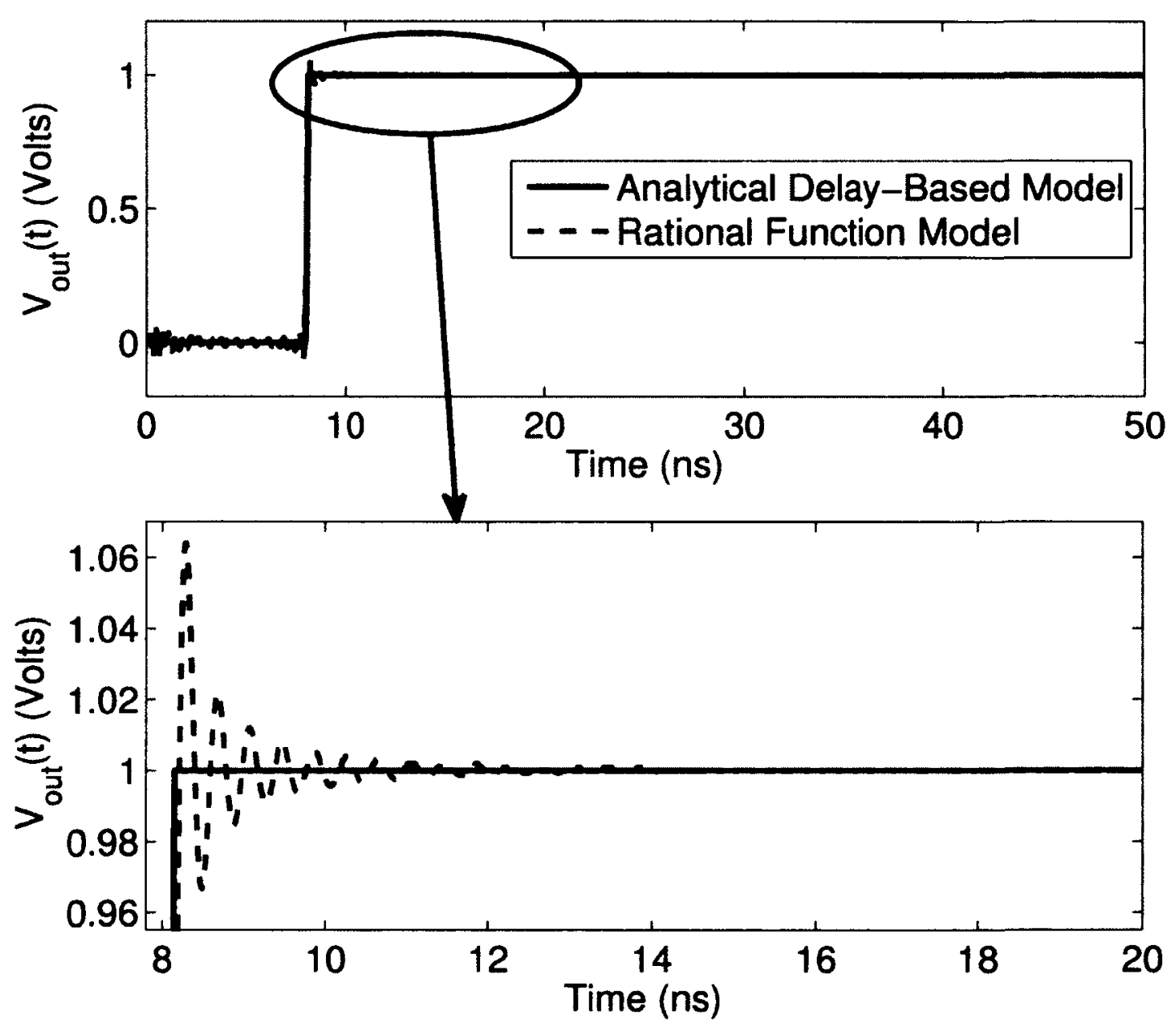

FIGURE 3.4: Illustration of spurious ringing effect after rising clock edge. 


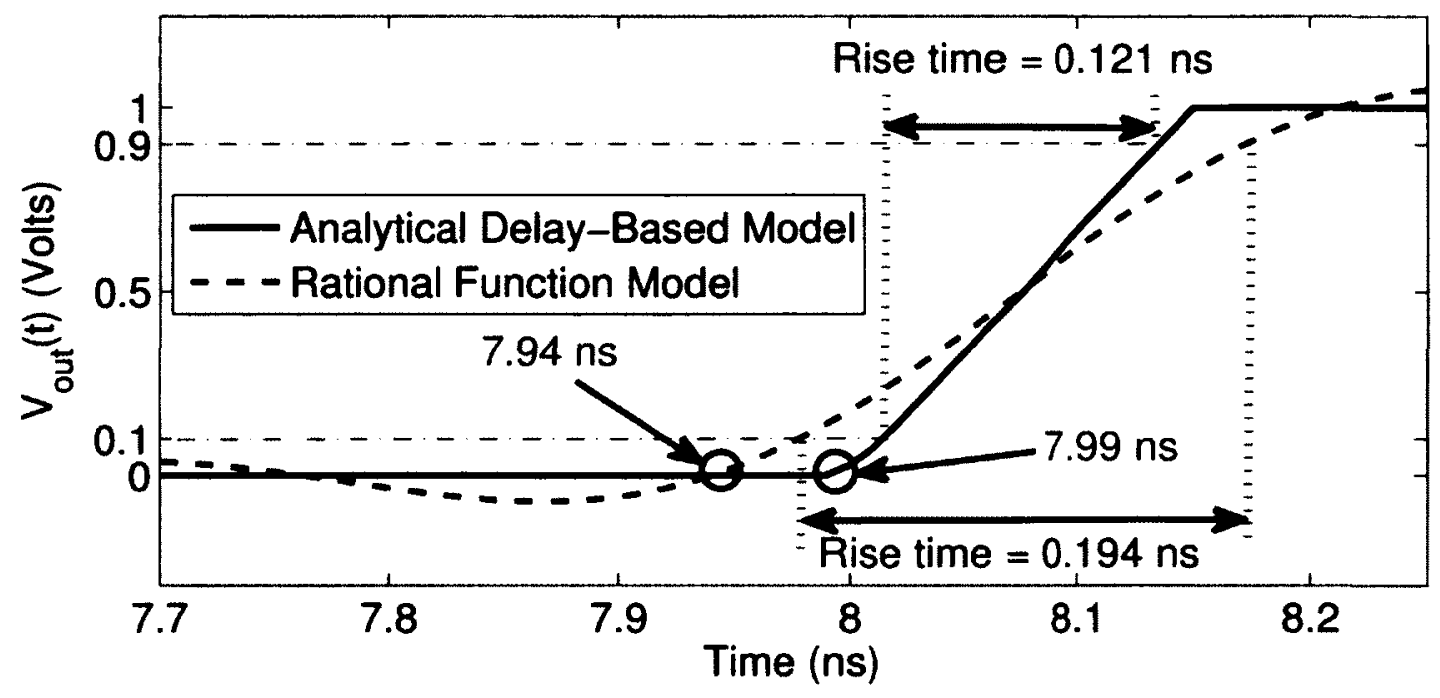

FIGURE 3.5: Illustration of rise time error.

switching speeds of a given device. For a designer, this error manifests itself in the timing analysis as unnecessarily large setup and hold times. To accommodate the wider timing constraints from the hundreds or thousands of components (each requiring a separate macromodel), it will force the designer to devise a slower overall product.

\subsection{Macromodeling Techniques for Time-Delay Networks}

As discussed in the previous section, macromodeling electrically long tabulated data networks using rational functions can yield high-order approximations and inaccurate time-domain analysis. To address this issue, efficient delay-extraction based techniques have been developed for tabulated data networks with long time-delays [26]-[34]. Each of these methods approximates the tabulated data using Delayed 
Rational Functions (DRFs) in the form

$$
H(s)=\sum_{m=0}^{M} Q_{m}(s) e^{-s \tau_{m}}
$$

where $Q_{m}(s)$ are rational functions, $M$ is the total number of delays in the network, and $\tau_{m}$ is the $m^{\text {th }}$ delay with $\tau_{0}=0$. Expanding each of the $Q_{m}(s)$ in its rational form gives

$$
H(s)=\sum_{m=0}^{M} \overbrace{\left(r_{m, 0}+\sum_{n=1}^{N_{m}} \frac{r_{m, n}}{s-p_{m, n}}\right)}^{Q_{m}(s)} e^{-s \tau_{m}}
$$

where for $Q_{m}(s): N_{m}, r_{m, n}$, and $p_{m, n}$ are the total number of poles, the residues, and the poles, respectively. After approximating the tabulated data using DRFs, they are converted into an equivalent circuit for implementation in standard SPICE simulators.

Several techniques are available in the literature to obtain DRF approximations of general networks from tabulated data [26]-[34]. In [31]-[34] Hilbert transform based delay estimation was proposed for general networks characterized by tabulated frequency-domain data. These methods extract only one dominant delay and neglect higher order reflections. Hence, the corresponding macromodel may not be efficient in the practical case of data with multiple delays. In [26], [27], general DRF-based macromodeling techniques were developed for networks characterized by tabulated time-domain data with multiple delays. In [28], a Gabor transform based macromodeling technique was proposed and [29], [30] extended this technique to be formulated via delayed vector fitting. These methods were developed for networks with multiple delays characterized by tabulated frequency-domain data. Conceptually, algorithms 
in [26]-[30] are similar. Delays are first identified using time-frequency analysis and subsequently, an iterative least squares technique is applied to obtain an accurate estimation of the poles and residues for the delayed rational function.

The following sections provide details of the DRF approximation techniques for tabulated frequency-domain data relevant to this thesis. For a thorough review of time-domain DRF approximation algorithms, the interested reader is referred to [92].

\subsection{Delay-based Macromodeling via Hilbert}

\section{Transforms}

In this section, the Hilbert transform based macromodeling technique for tabulated frequency-domain networks is reviewed [31]-[34]. In this technique, using the properties of minimum phase for passive structures, the Hilbert transform is applied to obtain an estimate for the port-to-port delays in the network.

To begin, recall that a function is a minimum phase function if all its poles and zeros lie in the left-half plane. In multi-port stable networks, this property is only exhibited by the self-responses (or diagonal elements) of the system. Now consider the two-port network with a transfer function given by

$$
\boldsymbol{H}(s)=\left[\begin{array}{ll}
H_{11}(s) & H_{12}(s) \\
H_{21}(s) & H_{22}(s)
\end{array}\right]
$$

The off diagonal elements, which represent port-to-port functionality of the system, are not minimum phase functions, unlike the self-response $H_{11}(s)$ and $H_{22}(s)$. Next, recall that an all-pass function is defined as a function with magnitude of 1 over the 
entire frequency range. Using the fact that any stable system function can be written as a product of a minimum phase function and an all-pass function, $H_{12}(s)$ is written as

$$
H_{12}(s)=H_{\min }(s) H_{A P}(s)
$$

where $H_{\min }(s)$ is the minimum phase function and $H_{A P}(s)$ is the all-pass function. If $\tau$ is the delay between port 1 and 2 in the system, then $H_{12}(s)$ can also be written as

$$
H_{12}(s)=\tilde{H}_{12}(s) e^{-s \tau}
$$

Note that $e^{-s \tau}$ has magnitude of unity for $s=j \omega$ over the entire frequency range $\omega \in \mathbb{R}$. Therefore, it is selected as the all-pass function. This leaves $\tilde{H}_{12}(s)=H_{\min }(s)$ as the minimum phase function. Combining (3.12), (3.13) and the fact that $\tilde{H}_{12}(s)=$ $H_{\min }(s)$ yields

$$
H_{A P}(j \omega)=\frac{H_{12}(j \omega)}{H_{\min }(j \omega)}=e^{-j \omega \tau}
$$

Rearranging (3.14) to obtain an expression for the delay gives

$$
\tau=-\frac{\arg \left(H_{A P}(j \omega)\right)}{\omega}=-\frac{\arg \left(\frac{H_{12}(j \omega)}{H_{\min }(j \omega)}\right)}{\omega}
$$

It remains to find and expression for the minimum phase function $H_{\min }(j \omega)$ in terms of the original function $H_{12}(j \omega)$.

It is known that the magnitude and the phase of a minimum phase function are related by the Hilbert transform. This is given mathematically as

$$
\arg \left[H_{\min }(j \omega)\right]=-\frac{1}{2 \pi} \mathcal{P} \int_{-\pi}^{\pi} \log \left|H_{\min }(j \omega)\right| \cot \left(\frac{\omega-\theta}{2}\right) d \theta
$$


where $\mathcal{P}$ represents the Cauchy Principal value [93]. Next, recall that

$$
\left|\tilde{H}_{12}(j \omega)\right|=\left|H_{\min }(j \omega)\right|=\left|H_{12}(j \omega)\right|
$$

where $|\cdot|$ is the absolute value of the argument. Substituting (3.17), (3.16), and $H_{\min }(j \omega)=\left|H_{\min }(j \omega)\right| e^{j \arg \left[H_{\min }(j \omega)\right]}$ into (3.15), the delay can be expressed in terms of $H_{12}(j \omega)$ as

$$
\tau=-\frac{\arg \left(\frac{H_{12}(j \omega)}{\left|H_{12}(j \omega)\right| e^{\frac{-j}{2 \pi} \mathcal{P} \int_{-\pi}^{\pi} \log \left|H_{12}(j \omega)\right| \cot \left(\frac{\omega-\theta}{2}\right) d \theta}}\right)}{\omega}
$$

Once the delay has been estimated, transient simulation is carried out using signal flow graph techniques [34].

\subsection{Delay-based Macromodeling via Gabor Trans-}

\section{forms}

In this section, the Gabor transform based macromodeling technique for networks characterized by tabulated frequency-domain data is reviewed [28]. In this algorithm, the delays are first estimated using time-frequency analysis. Next, the frequencydomain transfer function data is converted into separate subsets via time-frequency transforms such that, each subset contains the frequency-domain data for a single delayed impulse function. Then, each subset of data is fit separately using the vector fitting algorithm coupled with an optimization process to identify the optimal timedelay. Combining the approximations obtained for each of the separate subsets gives 
a Delayed Rational Function (DRF) approximation for the original data.

In this section, first, details of the delay estimation process are given. Next, the methodology for constructing DRF approximations using the Gabor transform is reviewed. For simplicity, the analysis provided in this section is based on continuoustime transformations. However, in practice the equivalent discrete-time transformations are used to handle bandlimited tabulated data.

\subsubsection{Delay Estimation using Time-Frequency Analysis}

Consider the scalar frequency-domain transfer function $H(\omega)$. Its corresponding timedomain impulse response can be considered a series of attenuated impulse functions, each of which is delayed in time. The delays in the network correspond to the timedomain locations of these impulses. Therefore, identification of the time-delays reduces to finding the locations of the impulses.

Recall that impulses in the time-domain correspond to localized regions of highfrequency or energy content. Prominent methodologies to localize frequency content in time are based on windowed Fourier transforms [94]. Here, since the original function is given in the frequency-domain, a windowed inverse Fourier transform is used. For $H(\omega)$, this inverse transform is given by

$$
\mathcal{G}(\omega, t)=\int_{-\infty}^{+\infty} H(\varepsilon) W^{*}(\varepsilon-\omega) e^{j \epsilon t} d \varepsilon
$$

where $t$ is the time variable and $W(\varepsilon)$ is the windowing function. One such windowing function is the Gaussian defined by

$$
W(\varepsilon)=\pi^{-1 / 4} e^{-\varepsilon^{2} / 2}
$$


A windowed Fourier transform using this Gaussian windowing function is sometimes referred to as the Gabor transform [28]. The energy density function or spectrogram corresponding to the Gabor transform is given by

$$
P_{s}(\omega, t)=|\mathcal{G}(\omega, t)|^{2}=\left|\int_{-\infty}^{+\infty} H(\varepsilon) W^{*}(\varepsilon-\omega) e^{j \epsilon t} d \varepsilon\right|^{2}
$$

The function $P_{s}(\omega, t)$ gives the energy content of $H(\omega)$ in the neighborhood of $(\omega, t)$. From this energy function, it is desired to identify the locations in time with the highest energy or frequency content. For this purpose, the average of the energy density function over all frequencies is calculated as

$$
\xi(t)=\frac{1}{2 \pi} \int_{-\infty}^{+\infty} P_{s}(\omega, t) d \omega=\frac{1}{2 \pi} \int_{-\infty}^{+\infty}|\mathcal{G}(\omega, t)|^{2} d \omega
$$

Then, the location of the maxima in the average energy density function, $\xi(t)$, provide an estimation for the time-delays $\left(\hat{\tau}_{1}, \hat{\tau}_{2}, \ldots, \hat{\tau}_{K}\right)$ in the response $H(\omega)$.

\subsubsection{Construction of Delayed Rational Functions (DRFs)}

Recall, the original function $H(\varepsilon)$ can be recovered from the Gabor transform using the inversion formula given by

$$
H(\varepsilon)=\frac{1}{2 \pi} \int_{-\infty}^{+\infty} \int_{-\infty}^{+\infty} \mathcal{G}(\omega, t) W(\varepsilon-\omega) e^{-j \varepsilon t} d \omega d t
$$


where $\mathcal{G}(\omega, t)$ is Gabor transform defined in (3.19) - (3.20). An important property of the inversion formula is that, it can be partitioned into separate integrals such that

$$
H(\varepsilon)=\sum_{k=1}^{K} H_{k}(\varepsilon), \quad H_{k}(\varepsilon)=\frac{1}{2 \pi} \int_{-\infty}^{+\infty} \int_{T_{k}}^{T_{k+1}} \mathcal{G}(\omega, t) W(\varepsilon-\omega) e^{-j \varepsilon t} d \omega d t
$$

where $T_{1}=-\infty, T_{K+1}=\infty$, and the remaining $\left\{T_{k}\right\}$ correspond to partition points along the time axis. The partition points are chosen as the minima between each pair of maxima, $\left\{\hat{\tau}_{k}\right\}$, found during the delay estimation process. By splitting the integral in this manner, each frequency-domain transfer function, $H_{k}(\varepsilon)$, corresponding to the time interval $\left(T_{k}, T_{k+1}\right)$ contains a single impulse function.

Next, consider the delay-free transfer function for each interval

$$
\tilde{H}_{k}(\varepsilon)=H_{k}(\varepsilon) e^{+j \varepsilon \tau_{k}}
$$

where $\tau_{k}$ is the true delay on $\left(T_{k}, T_{k+1}\right) . \quad \tilde{H}_{k}(\varepsilon)$ is approximated with a rational function using the vector fitting algorithm. The fitting is coupled with an optimization process to identify the true delay on $\left(T_{k}, T_{k+1}\right)$ [54], [55], with the optimization region chosen as a small interval centered at $\hat{\tau}_{k}$. Since the $\tilde{H}_{k}(\varepsilon)$ do not contain delay, the vector fitting algorithm yields low-order rational function approximations in the form

$$
\tilde{H}_{k}(\varepsilon)=\sum_{m=1}^{M_{k}} \frac{r_{m, k}}{j \varepsilon-p_{m, k}}
$$

where for the $k^{t h}$ function $\tilde{H}_{k}(\varepsilon): M_{k}$ is the number of poles, $\left\{r_{m, k}\right\}$ are the residues, 
and $\left\{p_{m, k}\right\}$ are the poles. Combining the results in (3.26) with (3.24) gives

$$
H(\varepsilon)=\sum_{k=1}^{K} \tilde{H}_{k}(\varepsilon) e^{-j \varepsilon \tau_{k}}=\sum_{k=1}^{K}\left(\sum_{m=1}^{M_{k}} \frac{r_{m, k}}{j \varepsilon-p_{m, k}}\right) e^{-j \varepsilon \tau_{k}}
$$

From (3.27), it is clear that the original transfer function is now approximated with a DRF.

\subsection{The Delayed Vector Fitting Algorithm}

In [29], [30], two fitting techniques were presented extending the Gabor transformbased methods [28] presented in the previous section. These algorithms modify the iterative global least squares techniques known as the Sanathanan-Koerner iteration [13] and vector fitting [17] (presented in Section 2.2.1) for the case of delayed rational functions. In this section, the more robust and stable extension based on vector fitting, termed delayed vector fitting is reviewed.

The delayed vector fitting algorithm begins with a function represented by a set of $N$ data points, $\{f(s)\}=\left\{f\left(s_{1}\right), f\left(s_{2}\right), \ldots, f\left(s_{N}\right)\right\}$, and builds a Delayed Rational Function (DRF) approximation of the form

$$
f(s)=\sum_{k=1}^{K}\left[\sum_{m=1}^{M}\left(\frac{r_{m, k}}{s-p_{m}}+d_{k}\right) e^{-s \tau_{k}}\right]
$$

where $M$ is the total number of poles, $\left\{p_{m}\right\}$ is the set of poles, and $K$ is the total number of delays. For the $k^{\text {th }}$ delay, $\tau_{k}: r_{m, k}$ are real or come in complex conjugate pairs representing the residues (corresponding to the poles $p_{m}$ ) and the values $d_{k}$ are real. The goal of the delayed vector fitting algorithm is to estimate all the delays, 
residues, and poles such that (3.28) provides an accurate approximation (in the least squares sense) of the given tabulated data.

The delayed vector fitting algorithm first obtains an estimate for the delays using the Gabor transform and time-frequency analysis [28] (discussed in Section 3.4). Next, it follows the same two-stage process as vector fitting (discussed in Section 2.2.1) to determine the remaining unknown poles and residues in (3.28). Stage one makes an initial guess at the poles over the frequency interval of interest. Next, it solves a least squares problem for the scaling function, $\sigma(s)$, whose zeros become the new initial set of poles for the system. This process is repeated iteratively until convergence is achieved. Stage two then calculates the residues of (3.28) using the final set of poles obtained in stage one. Further details are given in the following sections.

\subsubsection{Stage 1: Pole Identification}

The algorithm begins by choosing a set of starting poles, $\left\{\bar{p}_{m}\right\}$ (for details see Section 2.2.1). Following this, a scaling function, $\sigma(s)$, is defined such that

$$
\sigma(s)=\sum_{m=1}^{M} \frac{\tilde{r}_{m}}{s-\bar{p}_{m}}+1
$$

where $\tilde{r}_{m}$ are the residues of $\sigma(s)$. Next, a scaled function is defined such that

$$
\sigma(s) f(s) \approx \hat{f}(s)=\sum_{k=1}^{K} \sum_{m=1}^{M}\left(\frac{\hat{r}_{m, k}}{s-\bar{p}_{m}}+\hat{d}_{k}\right) e^{-s \tau_{k}}
$$

Equations (3.29) and (3.30) are combined to obtain

$$
\left[\sum_{k=1}^{K} \sum_{m=1}^{M}\left(\frac{\hat{r}_{m, k}}{s-\bar{p}_{m}}+\hat{d}_{k}\right) e^{-s \tau_{k}}\right] \approx\left[\sum_{m=1}^{M} \frac{\tilde{r}_{m}}{s-\bar{p}_{m}}+1\right] f(s)
$$


or

$$
\left[\sum_{k=1}^{K} \sum_{m=1}^{M}\left(\frac{\hat{r}_{m, k}}{s-\bar{p}_{m}}+\hat{d}_{k}\right) e^{-s \tau_{k}}\right]-\left[\sum_{m=1}^{M} \frac{\tilde{r}_{m}}{s-\bar{p}_{m}}\right] f(s)=f(s) .
$$

Writing (3.32) at $N$ frequency points, where $N \geq(K \times M+M+1)$ (the number of unknowns), gives an overdetermined linear least squares problem of the form $\boldsymbol{A x}=\boldsymbol{b}$ where the unknowns $\hat{r}_{m, k}, \hat{d}_{k}$, and $\tilde{r}_{m}$ are contained in the solution vector $\boldsymbol{x}$. In expanded form, this is written as

$$
\overbrace{\left[\begin{array}{llll}
\hat{\boldsymbol{A}}_{1} & \cdots & \hat{\boldsymbol{A}}_{K} & \tilde{\boldsymbol{A}}
\end{array}\right]}^{\boldsymbol{A}} \overbrace{\left[\begin{array}{c}
\hat{\boldsymbol{x}}_{1} \\
\vdots \\
\hat{\boldsymbol{x}}_{K} \\
\tilde{\boldsymbol{x}}
\end{array}\right]}^{\boldsymbol{x}}=\overbrace{\left[\begin{array}{c}
f\left(s_{1}\right) \\
f\left(s_{2}\right) \\
\vdots \\
f\left(s_{N}\right)
\end{array}\right]}^{\boldsymbol{b}}
$$

where

$$
\hat{\boldsymbol{A}}_{k}=\left[\begin{array}{cccc}
\frac{e^{-s_{1} \tau_{k}}}{s_{1}-\bar{p}_{1}} & \cdots & \frac{e^{-s_{1} \tau_{k}}}{s_{1}-\bar{p}_{M}} & 1 \\
\frac{e^{-s_{2} \tau_{k}}}{s_{2}-\bar{p}_{1}} & \cdots & \frac{e^{-s_{2} \tau_{k}}}{s_{2}-\bar{p}_{M}} & 1 \\
\vdots & \ddots & \vdots & \vdots \\
\frac{e^{-s_{N} \tau_{k}}}{s_{N}-\bar{p}_{1}} & \cdots & \frac{e^{-s_{N} \tau_{k}}}{s_{N}-\bar{p}_{M}} & 1
\end{array}\right], \tilde{\boldsymbol{A}}=\left[\begin{array}{ccc}
\frac{-f\left(s_{1}\right)}{s_{1}-\bar{p}_{1}} & \cdots & \frac{-f\left(s_{1}\right)}{s_{1}-\bar{p}_{M}} \\
\frac{-f\left(s_{2}\right)}{s_{2}-\bar{p}_{1}} & \cdots & \frac{-f\left(s_{2}\right)}{s_{2}-\bar{p}_{M}} \\
\vdots & \ddots & \vdots \\
\frac{-f\left(s_{N}\right)}{s_{N}-\bar{p}_{1}} & \cdots & \frac{-f\left(s_{N}\right)}{s_{N}-\bar{p}_{M}}
\end{array}\right], \hat{\boldsymbol{x}}_{k}=\left[\begin{array}{c}
\hat{r}_{1, k} \\
\vdots \\
\hat{r}_{M, k} \\
\hat{d}_{k}
\end{array}\right], \tilde{\boldsymbol{x}}=\left[\begin{array}{c}
\tilde{r}_{1} \\
\vdots \\
\tilde{r}_{M}
\end{array}\right]
$$

This system can be solved using the standard Householder algorithm [77].

Equation (3.32) is now used to obtain a delayed rational function approximation for the function $f(s)$. Let $\left\{\hat{z}_{m, k}\right\}$ and $\left\{\tilde{z}_{m}\right\}$ be the zeros of $\hat{f}(s)$ and $\sigma(s)$, respectively. 
Then, note that the partial fraction expansion of $\sigma(s)$ and $f(s)$ can be written as

$$
\hat{f}(s)=\frac{\sum_{k=1}^{K}\left(\prod_{m=1}^{M}\left(s-\hat{z}_{m, k}\right)\right) e^{-s \tau_{k}}}{\prod_{m=1}^{M}\left(s-\bar{p}_{m}\right)}, \quad \sigma(s)=\frac{\prod_{m=1}^{M}\left(s-\tilde{z}_{m}\right)}{\prod_{m=1}^{M}\left(s-\bar{p}_{m}\right)} .
$$

Substituting (3.35) into (3.30) and rearranging for $f(s)$ gives

$$
\begin{aligned}
f(s) & =\frac{\hat{f}(s)}{\sigma(s)}=\frac{\left[\sum_{k=1}^{K}\left(\prod_{m=1}^{M}\left(s-\hat{z}_{m, k}\right)\right) e^{-s \tau_{k}}\right] /\left[\prod_{m=1}^{M}\left(s-\bar{p}_{m}\right)\right]}{\left[\prod_{m=1}^{M}\left(s-\tilde{z}_{m}\right)\right] /\left[\prod_{m=1}^{M}\left(s-\bar{p}_{m}\right)\right]} \\
& =\frac{\left[\sum_{k=1}^{K}\left(\prod_{m=1}^{M}\left(s-\hat{z}_{m, k}\right)\right) e^{-s \tau_{k}}\right]}{\left[\prod_{m=1}^{M}\left(s-\tilde{z}_{m}\right)\right]} .
\end{aligned}
$$

Therefore, the zeros of $\sigma(s)$ represent a refined set of poles for the function $f(s)$. This stage is repeated with the zeros of $\sigma(s)$ taken as the new set of starting poles until the desired convergence criteria is obtained. In some instances, the poles determined using (3.36) will lie in the right-half plane, meaning they are unstable. This problem is overcome by inverting the signs of the real parts for the unstable poles and performing another iteration.

The determination of zeros is found using the same method as the rational function case, described in Section 2.2.1. In addition, to improve convergence the delayed vector fitting algorithm is typically implemented using the relaxed vector fitting condition introduced in [18] and described in Section 2.2.1. 


\subsubsection{Stage 2: Residue Identification}

In this stage, the residues of $f(s)$ are calculated using the final set of poles, $\left\{p_{m}\right\}$, found in stage one. For this purpose, an overdetermined least squares problem is constructed that solves for the residues in (3.28) at $N$ frequency points. In expanded form this is given as

$$
\overbrace{\left[\begin{array}{lll}
\hat{\boldsymbol{A}}_{1} & \cdots & \hat{\boldsymbol{A}}_{K}
\end{array}\right]}^{\boldsymbol{A}} \overbrace{\left[\begin{array}{c}
\hat{\boldsymbol{x}}_{1} \\
\vdots \\
\hat{\boldsymbol{x}}_{K}
\end{array}\right]}^{\boldsymbol{x}}=\overbrace{\left[\begin{array}{c}
f\left(s_{1}\right) \\
f\left(s_{2}\right) \\
\vdots \\
f\left(s_{N}\right)
\end{array}\right]}^{\boldsymbol{b}}
$$

where $\hat{\boldsymbol{A}}_{k}$ and $\hat{\boldsymbol{x}}_{\boldsymbol{k}}$ are defined in (3.34).

To ensure exact complex pole pairs, modifications analogous to those given in Section 2.2.1 can be applied. 


\section{CHAPTER 4}

\section{Proposed Passive Macromodeling}

\section{Algorithm for Scattering}

\section{Parameter Based Delayed Rational}

\section{Functions}

In this chapter, passivity verification and compensation algorithms are developed for macromodels described by Delayed Rational Functions (DRFs), obtained from tabulated scattering parameters. Section 4.1 introduces the topic and describes the motivation for the proposed algorithms. Section 4.2 reviews DRF-based macromodeling techniques and passivity issues. Next, Section 4.3 develops theoretical foundations and proofs for the proposed passivity verification algorithm. Section 4.4 develops a half-size Frequency-Dependent Generalized Eigenvalue Problem (FDGEP) for efficient passivity verification and is followed by Section 4.5, which describes the pro- 
cess for identification of passivity violation regions. Section 4.6 introduces the new passivity compensation algorithm for DRF-based macromodels. Numerical results validating the accuracy and efficiency of the proposed algorithms are given in Section 4.7 .

This chapter provides the first contribution of the thesis. The work in this chapter first appeared in [68]-[71].

\subsection{Introduction}

As discussed in the previous chapter, in the case of high-speed modules containing long delays (such as modules containing cables, printed circuit board traces, highspeed links, etc.), DRF-based macromodeling techniques have recently been developed [26]-[30]. For macromodel construction, the delays are initially found by performing a time-frequency analysis of the given tabulated data. Subsequently, a least squares fitting algorithm is used to obtain an accurate DRF model for the tabulated data.

However, DRF approximation algorithms do not guarantee the passivity of the final macromodel. Passivity of such macromodels is critical to the stability of the transient simulation of the tabulated data network along with the rest of the circuitry. Widely adopted passivity enforcement methods based on first-order perturbation may require numerous iterations, requiring at each iteration passivity verification and enforcement. In the case of simple rational function based macromodels, the above process is well established in the literature [36]-[49]. It is to be noted that the passivity verification is a computationally expensive step and it may not be robust if frequency-sweep based techniques are used. For passivity verification of simple rational function based macromodels, efficient and robust techniques based on evaluation of full and half-size 
Hamiltonian matrices can be found [35]-[41]. In the specific case of transmission line models, passivity verification and compensation algorithms have also been developed [50]-[53]. However, passivity verification and compensation involving DRF-based macromodels derived for general networks that are represented by tabulated data is still an open research topic.

In this chapter, passivity verification and compensation algorithms for DRF-based macromodels obtained from tabulated data in the scattering domain are developed. For this purpose, a new theorem is introduced for fast identification of regions of passivity violation based on the solution of a quasi-periodic Frequency-Dependent Generalized Eigenvalue Problem (FDGEP). The proposed theorem reduces the necessary passivity verification search region from the entire right-half of the complex plane to just a single finite interval along the imaginary axis. In addition, a new passivity compensation algorithm is developed to iteratively perturb the residues of nonpassive delayed rational macromodels using first-order perturbation theory.

\subsection{Problem Formulation}

Tabulated data characterizing a multi-port network can be in the form of admittance, impedance, hybrid, or scattering parameters. In this chapter, the tabulated data is considered to be given in the form of scattering parameters. Consider an $L$-port network with the scattering parameters described by

$$
\left[\begin{array}{cccc}
S_{11} & S_{12} & \cdots & S_{1 L} \\
\vdots & \vdots & \ddots & \vdots \\
S_{L 1} & S_{L 2} & \cdots & S_{L L}
\end{array}\right]\left[\begin{array}{c}
a_{1} \\
\vdots \\
a_{L}
\end{array}\right]=\left[\begin{array}{c}
b_{1} \\
\vdots \\
b_{L}
\end{array}\right]
$$


where $\left\{a_{i}\right\}$ are the incident power waves, $\left\{b_{i}\right\}$ are the reflective power waves, and $S_{i k}$ is the $(i, k)$ element of the scattering matrix. For macromodeling networks with long delays, the data for each matrix entry, $S_{i k}(s)$, is approximated by a DRF [26]-[30] in the form

$$
S_{i k}(s)=Q_{0}^{i, k}(s)+\sum_{m=1}^{M} Q_{m}^{i, k}(s) e^{-s \tau_{m}}
$$

where $(i, k) \in(1,2, \ldots, L)$ are the indices referring to the corresponding matrix element, $M$ is the total number of delays in the network, and $\tau_{m}$ is the $m^{\text {th }}$ delay. Each of the $Q_{m}^{i, k}(s), m=(0,1, \ldots, M)$, is given by a rational function in the form

$$
Q_{m}^{i, k}(s)=R_{m, 0}^{i, k}+\sum_{n=1}^{N_{m}^{i, k}} \frac{R_{m, n}^{i, k}}{s-p_{m, n}^{i, k}}
$$

where for each $Q_{m}^{i, k}(s): N_{m}^{i, k}, R_{m, n}^{i, k}$, and $p_{m, n}^{i, k}$ are the total number of poles, the residues, and the poles, respectively. To obtain (4.3) using techniques such as those in [26]-[30], the delays are initially found by performing a time-frequency analysis of the given tabulated data. Subsequently, a least squares fitting algorithm is used to obtain an accurate approximation of the tabulated data in the time or frequency-domain.

Stability of the DRFs can easily be ensured by forcing the poles to be in the lefthalf of the complex plane during the fitting process [17]. However, in order for the scattering model to be passive, a more complex set of conditions must be satisfied. Specifically, a network described by its scattering matrix, $\boldsymbol{S}(s)$, is known to be passive if and only if the following conditions are satisfied [95].

a) $\boldsymbol{S}(s)$ is analytic for all values of $s$ with $\mathfrak{R e}(s)>0$;

b) $S\left(s^{*}\right)=S^{*}(s)$, where the superscript ' $*$ ' is the complex conjugate operator; 
c) The norm of $\boldsymbol{S}(s)$ is less than 1 for all values of $s$ with $\mathfrak{R e}(s)>0$. That is

$$
\boldsymbol{z}^{H}\left[\boldsymbol{I}-\boldsymbol{S}^{H}(s) \boldsymbol{S}(s)\right] \boldsymbol{z} \geq 0
$$

for all complex values of $s$ with $\mathfrak{R e}(s)>0$ and for any arbitrary vector $\boldsymbol{z}$. Here, the superscript ' $H$ ' represents the Hermitian conjugate operator.

Using (4.2)-(4.3) an equivalent state-space formulation in the time-domain can be constructed $[27]$ as

$$
\begin{aligned}
\dot{\boldsymbol{x}}(t) & =\boldsymbol{A} \boldsymbol{x}(t)+\boldsymbol{B} \boldsymbol{a}(t) \\
\boldsymbol{b}(t) & =\sum_{m=0}^{M} \boldsymbol{C}_{m} \boldsymbol{x}\left(t-\tau_{m}\right)+\sum_{m=0}^{M} \boldsymbol{D}_{m} \boldsymbol{a}\left(t-\tau_{m}\right)
\end{aligned}
$$

where $\boldsymbol{x}(t) \in \mathbb{R}^{P}$ represents the state variables, $\boldsymbol{A} \in \mathbb{R}^{P \times P}, \boldsymbol{B} \in \mathbb{R}^{P \times L}, C_{m} \in \mathbb{R}^{L \times P}$, $\boldsymbol{D}_{m} \in \mathbb{R}^{L \times L}, P=(N \times M) \times L$, and $N=\sum_{i, k, m} N_{m}^{i, k}$.

As discussed in the Section 4.1, the above conditions can be computationally intensive to verify. A direct approach would require a frequency-sweep of the entire right-half plane. For the case of rational function based macromodels, passivity can be verified in closed-form by checking that a Hamiltonian matrix (derived from the state-space formulation of the rational macromodel) does not have any pure imaginary eigenvalues [41]. However, for DRF macromodels, the presence of the delay terms (in the form of exponential functions in the frequency-domain) makes this task more difficult.

Recently, in [50], for the specific case of 2-port DRF macromodels of per-unit-length RLCG parameter based transmission line interconnects, obtained via the method of characteristics with certain constraints, it was shown that the necessary search region 
for passivity verification could be reduced to only a finite interval along the imaginary axis (instead of the entire right-half plane). In [51], this work was advanced for multi-port transmission line interconnects with multiple delays, reducing passivity verification to solving a quasi-periodic FDGEP over a common period corresponding to a finite interval along the imaginary axis. However, a robust passivity verification in the case of DRF macromodels of tabulated data still remains a challenging task. In order to address this issue, in the following sections, computationally efficient algorithms for passivity verification for DRF macromodels of tabulated scattering parameter based networks are given.

\subsection{Proposed Passivity Verification Algorithm}

Conventional passivity verification approaches for DRF macromodels derived from tabulated scattering parameter data require a robust sweep of the entire right-half plane. In order to reduce the necessary search region and related computational efforts, the following algorithm is proposed.

To begin, it is shown that by imposing certain constraints on the DRFs generated from tabulated data networks, the necessary search region for passivity verification is reduced from the entire right-half plane to just the imaginary axis. Subsequently, a quasi-periodic FDGEP is developed such that, its solutions correspond to passivity violations in the DRF macromodel over a single finite period along the imaginary axis (rather than the entire imaginary axis). Details are given below. 


\subsubsection{Reduction of the Search Region to the Imaginary Axis}

In order to reduce the necessary search region for passivity violations from the entire right-half complex plane to just the imaginary axis, the following Lemma is introduced.

Lemma 4.1: If $Q_{m}^{i, k}(s)$ is asymptotically stable for all $(i, k) \in\{1,2, \ldots, L\}, m \in$ $(0,1, \ldots, M)$ and

$$
\lim _{s \rightarrow \infty} Q_{m}^{i, k}(s)=0, \quad \text { for } m \neq 0
$$

then $\boldsymbol{S}(s)$ defined by (4.2) satisfies the passivity conditions a) and b). In addition, condition c) is satisfied for all $\mathfrak{R e}(s)>0$ if it is satisfied on the imaginary axis. That is, (4.4) can be replaced by the equivalent condition

$$
\boldsymbol{z}^{H}\left[\boldsymbol{I}-\boldsymbol{S}^{H}(j \omega) \boldsymbol{S}(j \omega)\right] \boldsymbol{z} \geq 0, \quad \forall \omega \in \mathbb{R} \text { and } \forall \boldsymbol{z} \in \mathbb{C}^{L}
$$

In order to prove Lemma 4.1, the following results are needed [50], [96].

Proposition 4.2: The exponential function, $e^{-s \tau}$ satisfies the Cauchy-Riemann equations.

Proposition 4.3: If two functions, $\mathcal{G}(s)$ and $\mathcal{H}(s)$, each satisfy the Cauchy-Riemann equations, then

i) $\mathcal{G}(s)+\mathcal{H}(s)$ satisfies the Cauchy-Riemann equations;

ii) $\mathcal{G}(s) \cdot \mathcal{H}(s)$ satisfies the Cauchy-Riemann equations.

Theorem 4.4 (Maximum/Minimum modulus theorem): If a nonconstant function is analytic, continuous, and nonvanishing in a bounded domain $\Omega$ and continu- 
ous along its contour $\mathcal{C}$, then the real part of this function attains its maximum and minimum values on the contour $\mathcal{C}$.

Proof of Lemma 4.1: It is required to prove, under the assumptions of Lemma 4.1 , that $\boldsymbol{S}(s)$ satisfies the passivity conditions $a), b$ ), and $c$ ).

To begin, note that the condition $b$ ) is obviously satisfied for a DRF with real coefficients (as all the complex poles are considered along with their conjugates). For condition a), we need to show that $\boldsymbol{S}(s)$ satisfies the Cauchy-Riemann equations [96] and has continuous partial derivatives in the right-half plane. For this purpose, we make use of propositions 4.2 and 4.3 .

Under the assumptions given in the Lemma, each of the rational functions $Q_{m}^{i, k}(s)$ is asymptotically stable (i.e. does not have any poles in the right-half plane). Consequently, each $Q_{m}^{i, k}(s)$ is analytic and satisfies the Cauchy-Riemann equations. From Proposition 4.2, $e^{-s \tau_{m}}$ also satisfies the Cauchy-Riemann equations. Then, noting that each scattering matrix element, $S_{i k}(s)$, is a sum of products of functions that satisfy the Cauchy-Riemann equations, it is obvious from Proposition 4.3 that $\boldsymbol{S}(s)$ described by (4.2) satisfies the Cauchy-Riemann equations. Therefore, since $\boldsymbol{S}(s)$ also has no singular points in the right-half plane, it is analytic for $\mathfrak{R e}(s)>0$ and condition a) is satisfied.

The remainder of this section focuses on proving condition c) of Lemma 4.1. Specifically, we seek to prove that if $\left[\boldsymbol{I}-\boldsymbol{S}^{\boldsymbol{H}}(s) \boldsymbol{S}(s)\right]$ is nonnegative definite on the $j \omega$-axis, then it must be nonnegative definite $\forall s \in \mathfrak{R e}(s)>0$. In order to accomplish this, we make use of the maximum/minimum modulus principles (Theorem 4.4).

For the purpose of this proof, let $\Omega$ represent a semicircular area with its bounding contour $\mathcal{C}$ centered at the origin, its circular arc extending into the right-half of the complex plane, and its axis extending along the $j \omega$-axis. An illustration of this is 
shown in Fig. 4.1 for $s=\sigma+j \omega$. It is obvious that as the radius of $\mathcal{C}$ becomes arbitrarily large, $\Omega$ will extend to cover the right-half plane. Since $\boldsymbol{S}(s)$ is analytic in $\Omega$, then so is $\left[\boldsymbol{I}-\boldsymbol{S}^{H}(s) \boldsymbol{S}(s)\right]$ (this can easily be shown using the Cauchy-Riemann equations and Propositions 4.2 and 4.3). Consequently, $\boldsymbol{z}^{H}\left[\boldsymbol{I}-\boldsymbol{S}^{H}(s) \boldsymbol{S}(s)\right] \boldsymbol{z}$ is also analytic for any arbitrary vector $z \in \mathbb{C}^{L}$. In addition, note that

$$
\mathfrak{R e}_{\mathfrak{e}}\left\{\boldsymbol{z}^{H}\left[\boldsymbol{I}-\boldsymbol{S}^{H}(s) \boldsymbol{S}(s)\right] \boldsymbol{z}\right\}=\boldsymbol{z}^{H} \boldsymbol{I} \boldsymbol{z}-\boldsymbol{z}^{H} \boldsymbol{S}^{H}(s) \boldsymbol{S}(s) \boldsymbol{z}
$$

and $\boldsymbol{z}^{* T} \boldsymbol{S}^{H}(s) \boldsymbol{S}(s) \boldsymbol{z}$ is a sum of magnitudes squared. Then, by the maximum/minimum modulus principle, the minimum value of $\boldsymbol{z}^{* T}\left[\boldsymbol{I}-\boldsymbol{S}^{H}(s) \boldsymbol{S}(s)\right] \boldsymbol{z}$ in $s \in \Omega$ occurs on $s \in \mathcal{C}$. Hence, if $\left[\boldsymbol{I}-\boldsymbol{S}^{H}(s) \boldsymbol{S}(s)\right]$ is nonnegative definite for $s \in \mathcal{C}$, then it must be nonnegative definite for all $s \in \Omega$. To summarize, it is possible to check that $\boldsymbol{z}^{* T}\left[\boldsymbol{I}-\boldsymbol{S}^{H}(s) \boldsymbol{S}(s)\right] \boldsymbol{z}$ is nonnegative definite in $\Omega$ by verifying it is nonnegative definite on the bounding contour $\mathcal{C}$. Next, we show that as the radius of $\mathcal{C}$ approaches $\infty$, it is only necessary to check the part of $\mathcal{C}$ that extends on the $j \omega$-axis.

We note that if each of the $Q_{m}^{i, k}(s)$ is strict rational for $m>0$ (i.e. for $Q_{m}^{i, k}(s)$ corresponding to non-zero delays $\tau_{m}$ ), then

$$
\left.S_{i k}(s)\right|_{s \rightarrow \infty}=\left.Q_{0}^{i, k}(s)\right|_{s \rightarrow \infty}
$$

Therefore, as the radius of the contour $\mathcal{C}$ approaches $\infty$, the behavior of each of the $S_{i k}(s)$ becomes asymptotically similar to the stable rational function $Q_{0}^{i, k}(s)$. Consequently, the scattering matrix $\boldsymbol{S}(s)$ becomes asymptotically independent of $\mathfrak{R e}(s)$. This leads to the result

$$
\left.\left[\boldsymbol{I}-\boldsymbol{S}^{\boldsymbol{H}}(s) \boldsymbol{S}(s)\right]\right|_{s \rightarrow \infty}=\left.\left[\boldsymbol{I}-\boldsymbol{S}^{H}(s) \boldsymbol{S}(s)\right]\right|_{s \rightarrow j \infty}
$$




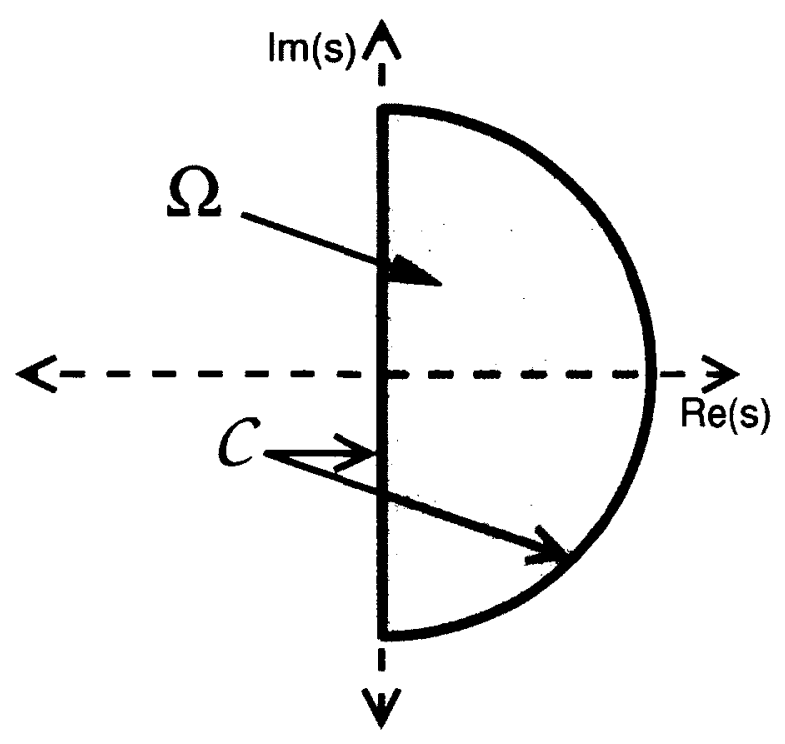

FIGURE 4.1: Semicircular arc in the right-half of the complex plane.

Equivalently, $\left[\boldsymbol{I}-\boldsymbol{S}^{H}(s) \boldsymbol{S}(s)\right]$ on the semi-circular arc of $\mathcal{C}$ approaches a limiting value equivalent to the limiting value obtained as $s \rightarrow j \infty$. Since the minimum value occurs on $\mathcal{C}$, then $\boldsymbol{z}^{* T}\left[\boldsymbol{I}-\boldsymbol{S}^{H}(s) \boldsymbol{S}(s)\right] \boldsymbol{z} \geq 0 \quad \forall s \mid \mathfrak{R e}(s)=0$ implies that $\boldsymbol{z}^{* T}[\boldsymbol{I}-$ $\left.\boldsymbol{S}^{H}(s) \boldsymbol{S}(s)\right] \boldsymbol{z} \geq 0 \quad \forall s \mid \mathfrak{R e}(s)>0$. This completes the proof of Lemma 4.1.

Lemma 4.1 requires that $R_{m, 0}^{i, k}=0, \forall m>0$ (i.e. all the direct coupling constants associated with delays greater than 0 vanish). Equivalently, imposing these conditions on (4.5) gives $\boldsymbol{D}_{\boldsymbol{m}}=\mathbf{0}, \forall m>0$. If we let $\boldsymbol{D}=\boldsymbol{D}_{0}$ and convert the set of state-space equations to the Laplace-domain, the scattering matrix becomes

$$
\boldsymbol{S}(s)=\left(\sum_{m=0}^{M} \boldsymbol{C}_{m} e^{-s \tau_{m}}\right)(s \boldsymbol{I}-\boldsymbol{A})^{-1} \boldsymbol{B}+\boldsymbol{D} .
$$

In addition, condition (4.7) at $\omega=\infty$ yields the constraint $\|\boldsymbol{D}\|<1$ (as in the case of conventional rational macromodels). 


\subsubsection{Reduction of the Search Region to a Single Finite Pe- riod on the Imaginary Axis}

It is to be noted that Lemma 4.1 reduces the search task substantially by restricting the search region from the entire right-half plane to just the imaginary axis. However, this is still a computationally expensive task. In order to further reduce the computational efforts, a quasi-periodic FDGEP is derived from the state-space formulation in (4.11). Subsequently, the FDGEP is solved over a single finite region to locate any passivity violations in the DRF macromodel. For this purpose, the following Theorem is developed.

Theorem 4.5: A DRF macromodel constructed from tabulated scattering parameter based data is passive if and only if there does not exist any purely imaginary eigenvalues for $s$ that satisfy the following frequency-dependent generalized eigenvalue problem

$$
s \boldsymbol{\zeta}=\boldsymbol{H}(s) \boldsymbol{\zeta}
$$

where

$$
\boldsymbol{H}(s)=\mathcal{V}+\sum_{m=0}^{M} \hat{\boldsymbol{W}}_{m} e^{-s \tau_{m}}+\sum_{m=0}^{M} \overline{\boldsymbol{W}}_{m} e^{s \tau_{m}}+\sum_{m, n=0}^{M} \boldsymbol{W}_{m n} e^{s\left(\tau_{m}-\tau_{n}\right)}
$$

and

$$
\mathcal{V}=\left[\begin{array}{cccc}
-\boldsymbol{A}^{T} & \mathbf{0} & \mathbf{0} & \mathbf{0} \\
\boldsymbol{B R} \boldsymbol{B}^{T} & \boldsymbol{A} & \mathbf{0} & \boldsymbol{B} \boldsymbol{R} \boldsymbol{B}^{T} \\
\boldsymbol{B R} \boldsymbol{B}^{T} & \mathbf{0} & \boldsymbol{A} & \boldsymbol{B} \boldsymbol{R} \boldsymbol{B}^{T} \\
\mathbf{0} & \mathbf{0} & \mathbf{0} & -\boldsymbol{A}^{T}
\end{array}\right], \boldsymbol{W}_{m n}=\left[\begin{array}{cccc}
0 & -C_{m}^{T} \boldsymbol{D R} D^{T} C_{n} & 0 & 0 \\
0 & 0 & 0 & 0 \\
0 & 0 & 0 & 0 \\
0 & 0 & -C_{m}^{T} C_{n} & 0
\end{array}\right]
$$




$$
\begin{aligned}
& \hat{\boldsymbol{W}}_{m}=\left[\begin{array}{ccccc}
\mathbf{0} & \mathbf{0} & \mathbf{0} & 0 \\
\mathbf{0} & \boldsymbol{B} \boldsymbol{R} \boldsymbol{D}^{T} \boldsymbol{C}_{m} & \mathbf{0} & \mathbf{0} \\
\mathbf{0} & \boldsymbol{B} \boldsymbol{R} \boldsymbol{D}^{T} \boldsymbol{C}_{m} & \mathbf{0} & \mathbf{0} \\
\mathbf{0} & 0 & \mathbf{0} & \mathbf{0}
\end{array}\right], \overline{\boldsymbol{W}}_{m}=\left[\begin{array}{cccc}
-C_{m}^{T} \boldsymbol{D} \boldsymbol{R} \boldsymbol{B}^{T} & \mathbf{0} & \mathbf{0} & -C_{m}^{T} \boldsymbol{D R} \boldsymbol{B}^{T} \\
0 & 0 & 0 & 0 \\
0 & 0 & 0 & 0 \\
0 & 0 & 0 & 0
\end{array}\right] \\
& \boldsymbol{R}=\left(\boldsymbol{I}-\boldsymbol{D}^{T} \boldsymbol{D}\right)^{-\mathbf{1}} \text {. }
\end{aligned}
$$

Proof of Theorem 4.5: Lemma 4.1 shows that macromodels described by (4.11) become nonpassive when one (or more) of the eigenvalues of $\left[\boldsymbol{I}-\boldsymbol{S}^{H}(s) \boldsymbol{S}(s)\right]$ becomes negative for some value of $s$ on the $j \omega$-axis. Let the set of eigenvalues for $[\boldsymbol{I}-$ $\left.\boldsymbol{S}^{H}(s) \boldsymbol{S}(s)\right]$ be denoted by $\left\{\lambda_{k}(j \omega)\right\}$. In the remainder of this section, we give the proof that the necessary and sufficient conditions to have $\lambda_{k}\left(j \omega_{0}\right)=0$ for some $\omega_{0}$ is that $s_{0}=j \omega_{0}$ satisfies (4.12).

The necessity part implies that at $s_{0}=j \omega_{0}, \lambda_{k}\left(j \omega_{0}\right)=0$ and therefore, the matrix $\left[\boldsymbol{I}-\boldsymbol{S}^{H}(s) \boldsymbol{S}(s)\right]$ is singular. Equivalently

$$
\left[\boldsymbol{I}-\boldsymbol{S}^{H}(s) \boldsymbol{S}(s)\right] \boldsymbol{\eta}=\mathbf{0}
$$

where $\boldsymbol{\eta}$ is the corresponding eigenvector. Substituting (4.11) in (4.15) and noting $s_{0}^{*}=-s_{0}$ for $s_{0}=j \omega_{0}$ yields

$$
\begin{aligned}
\boldsymbol{\eta}=\boldsymbol{R}\left[\boldsymbol{D}^{T} \hat{\boldsymbol{C}}\left(s_{0}\right)\left(s_{0} \boldsymbol{I}-\boldsymbol{A}\right)^{-1} \boldsymbol{B}-\boldsymbol{B}^{T}\left(s_{0} \boldsymbol{I}+\boldsymbol{A}^{T}\right)^{-1} \hat{\boldsymbol{C}}^{T}\left(s_{0}^{*}\right) \boldsymbol{D}\right. \\
\left.-\boldsymbol{B}^{T}\left(s_{0} \boldsymbol{I}+\boldsymbol{A}^{T}\right)^{-1} \hat{\boldsymbol{C}}^{T}\left(s_{0}^{*}\right) \hat{\boldsymbol{C}}\left(s_{0}\right)\left(s_{0} \boldsymbol{I}-\boldsymbol{A}\right)^{-1} \boldsymbol{B}\right] \boldsymbol{\eta}
\end{aligned}
$$


with $\boldsymbol{R}$ defined in (4.14) and

$$
\hat{C}\left(s_{0}\right)=\sum_{m=0}^{M} C_{m} e^{-s_{0} \tau_{m}}
$$

Performing some simple mathematical manipulations yields

$$
\boldsymbol{\eta}=\boldsymbol{R} \boldsymbol{B}^{T} \boldsymbol{\delta}+\boldsymbol{R} \boldsymbol{D}^{T} \hat{\boldsymbol{C}}\left(s_{0}\right) \boldsymbol{\gamma}+\boldsymbol{R} \boldsymbol{B}^{T} \boldsymbol{\alpha}
$$

where

$$
\begin{aligned}
& \boldsymbol{\delta}=-\left(s_{0} \boldsymbol{I}+\boldsymbol{A}^{T}\right)^{-1} \hat{\boldsymbol{C}}^{T}\left(s_{0}^{*}\right) \boldsymbol{D} \boldsymbol{\eta} \\
& \boldsymbol{\gamma}=\left(s_{0} \boldsymbol{I}-\boldsymbol{A}\right)^{-1} \boldsymbol{B} \boldsymbol{\eta} \\
& \boldsymbol{\alpha}=-\left(s_{0} \boldsymbol{I}+\boldsymbol{A}^{T}\right)^{-1} \hat{\boldsymbol{C}}^{T}\left(s_{0}^{*}\right) \hat{\boldsymbol{C}}\left(s_{0}\right) \boldsymbol{\theta}
\end{aligned}
$$

and

$$
\boldsymbol{\theta}=\boldsymbol{\gamma}
$$

Rearranging (4.19a) - (4.19c), respectively, (for example, for (4.19a) multiplying both sides by the ( $\left.\boldsymbol{I}+\boldsymbol{A}^{T}\right)$ and moving $\boldsymbol{A}^{T} \boldsymbol{\delta}$ to the right hand side) we obtain the set of equations

$$
\begin{aligned}
s_{0} \boldsymbol{\delta} & =-\boldsymbol{A}^{T} \boldsymbol{\delta}-\hat{\boldsymbol{C}}^{T}\left(s_{0}^{*}\right) \boldsymbol{D} \boldsymbol{\eta} \\
s_{0} \boldsymbol{\gamma} & =\boldsymbol{A} \boldsymbol{\gamma}+\boldsymbol{B} \boldsymbol{\eta} \\
s_{0} \boldsymbol{\alpha} & =-\boldsymbol{A}^{T} \boldsymbol{\alpha}-\hat{\boldsymbol{C}}^{T}\left(s_{0}^{*}\right) \hat{\boldsymbol{C}}\left(s_{0}\right) \boldsymbol{\theta}
\end{aligned}
$$


Substituting (4.18) into (4.21a) - (4.21c), respectively, we obtain

$$
\begin{aligned}
& s_{0} \boldsymbol{\delta}=-\boldsymbol{A}^{T} \boldsymbol{\delta}-\hat{\boldsymbol{C}}^{T}\left(s_{0}^{*}\right) \boldsymbol{D} \boldsymbol{R} \boldsymbol{B}^{T} \boldsymbol{\delta}-\hat{\boldsymbol{C}}^{T}\left(s_{0}^{*}\right) \boldsymbol{D} \boldsymbol{R} \boldsymbol{D}^{T} \hat{\boldsymbol{C}}\left(s_{0}\right) \boldsymbol{\gamma}-\hat{\boldsymbol{C}}^{T}\left(s_{0}^{*}\right) \boldsymbol{D} \boldsymbol{R} \boldsymbol{B}^{T} \boldsymbol{\alpha} \\
& s_{0} \boldsymbol{\gamma}=\boldsymbol{A} \boldsymbol{\gamma}+\boldsymbol{B} \boldsymbol{R} \boldsymbol{B}^{T} \boldsymbol{\delta}+\boldsymbol{B} \boldsymbol{R} \boldsymbol{D}^{T} \hat{\boldsymbol{C}}\left(s_{0}\right) \boldsymbol{\gamma}+\boldsymbol{B} \boldsymbol{R} \boldsymbol{B}^{T} \boldsymbol{\alpha} \\
& s_{0} \boldsymbol{\alpha}=-\boldsymbol{A}^{T} \boldsymbol{\alpha}-\hat{\boldsymbol{C}}^{T}\left(s_{0}^{*}\right) \hat{\boldsymbol{C}}\left(s_{0}\right) \boldsymbol{\theta}
\end{aligned}
$$

Using (4.17) and $s_{0}^{*}=-s_{0}$ for $s_{0}=j \omega_{0}$ we obtain

$$
\begin{aligned}
s_{0} \delta= & -\boldsymbol{A}^{T} \boldsymbol{\delta}-\sum_{m=0}^{M} C_{m}^{T} \boldsymbol{D} \boldsymbol{R} \boldsymbol{B}^{T} \boldsymbol{\delta} e^{s_{0} \tau_{m}}-\sum_{m=0}^{M} C_{m}^{T} \boldsymbol{D} \boldsymbol{R} \boldsymbol{D}^{T} \sum_{n=0}^{M} C_{n} \gamma e^{s_{0}\left(\tau_{m}-\tau_{n}\right)} \\
& -\sum_{m=0}^{M} \boldsymbol{C}_{m}^{T} \boldsymbol{D} \boldsymbol{R} \boldsymbol{B}^{T} \boldsymbol{\alpha} e^{s_{0} \tau_{m}} \\
s_{0} \boldsymbol{\gamma}= & \boldsymbol{A} \boldsymbol{\gamma}+\boldsymbol{B} \boldsymbol{R} \boldsymbol{B}^{T} \boldsymbol{\delta}+\boldsymbol{B} \boldsymbol{R} \boldsymbol{D}^{T} \sum_{m=0}^{M} \boldsymbol{C}_{m} \gamma e^{-s_{0} \tau_{m}}+\boldsymbol{B} \boldsymbol{R} \boldsymbol{B}^{T} \boldsymbol{\alpha} \\
s_{0} \boldsymbol{\alpha}= & -\boldsymbol{A}^{T} \boldsymbol{\alpha}-\sum_{m=0}^{M} \boldsymbol{C}_{m}^{T} \sum_{n=0}^{M} \boldsymbol{C}_{n} \boldsymbol{\theta} e^{s_{0}\left(\tau_{m}-\tau_{n}\right)} .
\end{aligned}
$$

Writing this system in the matrix form with

$$
\zeta=\left[\begin{array}{llll}
\delta^{T} & \gamma^{T} & \boldsymbol{\theta}^{T} & \alpha^{T}
\end{array}\right]^{T}
$$

gives (4.12). The above analysis proves the necessity condition.

The sufficiency of Theorem 4.5 can be proven by assuming that $s_{0}=j \omega_{0}$ satisfies (4.12) and working in the reverse manner to show that $\left[\boldsymbol{I}-\boldsymbol{S}^{H}(s) \boldsymbol{S}(s)\right]$ is singular. From the above analysis, it is obvious that if there are no imaginary eigenvalues satisfying (4.12), then there are no imaginary eigenvalues that satisfy $\left[\boldsymbol{I}-\boldsymbol{S}^{H}(s) \boldsymbol{S}(s)\right]=0$ 
(i.e. $\left.\left\{\lambda_{k}(j \omega) \neq 0 \quad \forall \omega \in \mathbb{R}\right\}\right)$. This implies that if the eigenvalues, $\left\{\lambda_{k}(j \omega)\right\}$, are positive for some values of $\omega$, then they are positive for all values of $\omega$. Therefore, $\left[\boldsymbol{I}-\boldsymbol{S}^{H}(s) \boldsymbol{S}(s)\right]$ has only positive eigenvalues, $\boldsymbol{S}(s)$ is bounded real, and consequently, $\boldsymbol{S}(s)$ is passive. This completes the proof.

Solving the above FDGEP requires performing matrix calculations using a $4 P \times 4 P$ matrix, where $P \times P$ is the size of the square state-space matrix $\boldsymbol{A}$. Consequently, this becomes an extremely CPU intensive task. In order to address this issue, the next section develops a reduced FDGEP which is half the size of the FDGEP in Theorem 4.5 .

\subsection{Development of a Half-Size FDGEP for Pas- sivity Verification}

To further minimize the computational effort in the passivity verification process, the following theorem for a half-size FDGEP is developed [70], [71].

Theorem 4.6: A DRF macromodel is passive if and only if there does not exist any purely imaginary eigenvalues for $s$ that satisfy the following modified frequencydependent generalized eigenvalue problem

$$
s \boldsymbol{\chi}=\mathcal{M}(s) \boldsymbol{\chi}
$$

where

$$
\mathcal{M}(s)=\mathcal{M}_{0}+\sum_{n=0}^{M} \hat{\mathcal{M}}_{n} e^{-s \tau_{n}}+\sum_{m=0}^{M} \overline{\mathcal{M}}_{m} e^{s \tau_{m}}+\sum_{m, n=0}^{M} \tilde{\mathcal{M}}_{m n} e^{s\left(\tau_{m}-\tau_{n}\right)}
$$


and

$$
\begin{array}{rlrl}
\mathcal{M}_{0} & =\left[\begin{array}{cc}
\boldsymbol{A} & \boldsymbol{B} \boldsymbol{R} \boldsymbol{B}^{T} \\
\mathbf{0} & -\boldsymbol{A}^{T}
\end{array}\right], & \hat{\mathcal{M}}_{n}=\left[\begin{array}{cc}
\boldsymbol{B R} \boldsymbol{D}^{T} \boldsymbol{C}_{n} & \mathbf{0} \\
\mathbf{0} & \mathbf{0}
\end{array}\right], \\
\overline{\mathcal{M}}_{m}=\left[\begin{array}{cc}
\mathbf{0} & \mathbf{0} \\
\mathbf{0} & -\boldsymbol{C}_{m}^{T} \boldsymbol{D} \boldsymbol{R} \boldsymbol{B}^{T}
\end{array}\right], & \tilde{\mathcal{M}}_{m n}=\left[\begin{array}{cc}
0 & 0 \\
-C_{m}^{T} \hat{\boldsymbol{R}} C_{n} & 0
\end{array}\right], \\
\boldsymbol{R} & =\left(\boldsymbol{I}-\boldsymbol{D}^{T} \boldsymbol{D}\right)^{-1}, & \hat{\boldsymbol{R}} & =\left(\boldsymbol{I}-\boldsymbol{D} \boldsymbol{D}^{T}\right)^{-1}
\end{array}
$$

Proof of Theorem 4.6: To begin, the necessity part of Theorem 4.6 is proven. Let $S$ be the transfer function such that

$$
\boldsymbol{b}=\boldsymbol{S a}
$$

where $\boldsymbol{b}$ is the output (reflected power waves) and $\boldsymbol{a}$ is the unit input (incident power waves). Then, in order to be passive, we need [36]

$$
\boldsymbol{b}^{H} \boldsymbol{b} \leq 1
$$

or

$$
\boldsymbol{a}^{H} \boldsymbol{S}^{H}(\boldsymbol{S a}) \leq 1
$$

Let

$$
\tilde{b}=S^{H} S a=S^{H} b
$$

Substituting the definition of $b$ from (4.28) into (4.29), and using the fact that the 
necessity condition for Theorem 4.6 implies equality in (4.29), we get

$$
\boldsymbol{a}^{H} \boldsymbol{S}^{H} \boldsymbol{S a}-1=\boldsymbol{a}^{H}\left(\boldsymbol{S}^{H} \boldsymbol{S}-\boldsymbol{I}\right) \boldsymbol{a}=0 .
$$

For the nontrivial solution (i.e. $|a| \neq 0),(4.32)$ implies

$$
\left(\boldsymbol{S}^{H} \boldsymbol{S}-\boldsymbol{I}\right) \boldsymbol{a}=\mathbf{0} .
$$

Then, noting that $\tilde{b}=\boldsymbol{S}^{H} \boldsymbol{S a},(4.33)$ is written as

$$
\tilde{b}-\boldsymbol{a}=\mathbf{0}
$$

Writing the state-space form of $\boldsymbol{b}=\boldsymbol{S a}$ in the Laplace-domain and applying Lemma 4.1 (i.e. with $s=j \omega$ ) gives

$$
\begin{aligned}
j \omega \boldsymbol{x}_{1} & =\boldsymbol{A} \boldsymbol{x}_{1}+\boldsymbol{B a} \\
\boldsymbol{b} & =\sum_{n=0}^{M} \boldsymbol{C}_{n} e^{-s \tau_{n}} \boldsymbol{x}_{1}+\boldsymbol{D} \boldsymbol{a} .
\end{aligned}
$$

Subsequently, define the output $\tilde{b}$ of the transfer function $S^{H}$ operating on $\boldsymbol{b}$ (i.e. $\left.\tilde{b}=\boldsymbol{S}^{H} \boldsymbol{b}\right)$ in state-space form as

$$
\begin{aligned}
-j \omega \boldsymbol{x}_{2} & =\boldsymbol{A}^{H} \boldsymbol{x}_{2}+\sum_{m=0}^{M} \boldsymbol{C}_{m}^{H} e^{s \tau_{m}} \boldsymbol{b} \\
\tilde{\boldsymbol{b}} & =\boldsymbol{B}^{H} \boldsymbol{x}_{2}+\boldsymbol{D}^{H} \boldsymbol{b} .
\end{aligned}
$$

Substituting (4.35b) into (4.36a), and combining (4.35b) with (4.36b) in (4.34) gives 
the following equations

$$
\begin{aligned}
j \omega \boldsymbol{x}_{2} & =-\boldsymbol{A}^{H} \boldsymbol{x}_{2}-\sum_{m=0}^{M} C_{m}^{H} e^{s \tau_{m}}\left(\sum_{n=0}^{M} \boldsymbol{C}_{n} e^{-s \tau_{n}} \boldsymbol{x}_{1}+\boldsymbol{D} \boldsymbol{a}\right) \\
0 & =\boldsymbol{B}^{H} \boldsymbol{x}_{2}+\boldsymbol{D}^{H}\left(\sum_{n=0}^{M} \boldsymbol{C}_{n} e^{-s \tau_{n}} \boldsymbol{x}_{1}+\boldsymbol{D} \boldsymbol{a}\right)-\boldsymbol{a} .
\end{aligned}
$$

Substituting $\boldsymbol{a}$ from (4.38) into each of (4.37) and (4.35a), and using $\boldsymbol{R}=(\boldsymbol{I}-$ $\left.D^{H} D\right)^{-1}$

$$
\begin{aligned}
j \omega \boldsymbol{x}_{1}= & \boldsymbol{A} \boldsymbol{x}_{1}+\boldsymbol{B} \boldsymbol{R} \boldsymbol{D}^{H} \sum_{n=0}^{M} \boldsymbol{C}_{n} e^{-s \tau_{n}} \boldsymbol{x}_{1}+\boldsymbol{B} \boldsymbol{R} \boldsymbol{B}^{H} \boldsymbol{x}_{2} \\
j \omega \boldsymbol{x}_{2}= & -\boldsymbol{A}^{H} \boldsymbol{x}_{2}-\sum_{m, n=0}^{M} \boldsymbol{C}_{m}^{H} e^{s\left(\tau_{m}-\tau_{n}\right)} \boldsymbol{C}_{n} \boldsymbol{x}_{1}-\sum_{m, n=0}^{M} \boldsymbol{C}_{m}^{H} e^{s\left(\tau_{m}-\tau_{n}\right)} \boldsymbol{D} \boldsymbol{R} \boldsymbol{D}^{H} \boldsymbol{C}_{n} \boldsymbol{x}_{1} \\
& -\sum_{m=0}^{M} \boldsymbol{C}_{m}^{H} e^{s \tau_{m}} \boldsymbol{D} \boldsymbol{R} \boldsymbol{B}^{H} \boldsymbol{x}_{2}
\end{aligned}
$$

Next, we define $\hat{\boldsymbol{R}}=\left(\boldsymbol{I}-\boldsymbol{D} \boldsymbol{D}^{H}\right)^{-1}=\boldsymbol{I}-\boldsymbol{D}\left(\boldsymbol{D}^{H} \boldsymbol{D}-\boldsymbol{I}\right)^{-1} \boldsymbol{D}^{H}$ and write the above system in matrix form to give

$$
\begin{gathered}
j \omega\left[\begin{array}{l}
\boldsymbol{x}_{1} \\
\boldsymbol{x}_{2}
\end{array}\right]=\left\{\left[\begin{array}{cc}
\boldsymbol{A} & \boldsymbol{B} \boldsymbol{R} \boldsymbol{B}^{H} \\
\mathbf{0} & -\boldsymbol{A}^{H}
\end{array}\right]+\sum_{n=0}^{M} e^{-s \tau_{n}}\left[\begin{array}{cc}
\boldsymbol{B R} \boldsymbol{D}^{H} \boldsymbol{C}_{n} & \mathbf{0} \\
0 & 0
\end{array}\right]\right. \\
\left.-\sum_{m=0}^{M} e^{s \tau_{m}}\left[\begin{array}{cc}
\mathbf{0} & \mathbf{0} \\
\mathbf{0} & \boldsymbol{C}_{m}^{H} \boldsymbol{D} \boldsymbol{R} \boldsymbol{B}^{H}
\end{array}\right]-\sum_{m, n=0}^{M} e^{s\left(\tau_{m}-\tau_{n}\right)}\left[\begin{array}{cc}
0 & 0 \\
C_{m}^{H} \hat{\boldsymbol{R}} \boldsymbol{C}_{n} & \mathbf{0}
\end{array}\right]\right\}\left[\begin{array}{l}
\boldsymbol{x}_{1} \\
\boldsymbol{x}_{2}
\end{array}\right] .
\end{gathered}
$$

Note that since each of the matrices contains only real values the Hermitian conjugate is equal to the matrix transpose. Then, defining $\boldsymbol{\chi}=\left[\begin{array}{ll}x_{1}^{H} & x_{2}^{H}\end{array}\right]^{H}$ and using the matrix definitions in (4.27), it is clear that (4.40) is equivalent to the FDGEP in (4.25). 
The sufficiency of Theorem 4.6 can be proven by assuming that $s_{0}=j \omega_{0}$ satisfies (4.25) and working in the reverse manner to show that $\left[\boldsymbol{I}-\boldsymbol{S}^{H}(s) \boldsymbol{S}(s)\right]$ is singular. From the above analysis, it is obvious that if there are no imaginary eigenvalues satisfying (4.25), then there are no imaginary eigenvalues that satisfy $\left[\boldsymbol{I}-\boldsymbol{S}^{H}(s) \boldsymbol{S}(s)\right]=0$ (i.e. $\left\{\lambda_{k}(j \omega) \neq 0 \quad \forall \omega \in \mathbb{R}\right\}$ ). This implies that if the eigenvalues of $\left[\boldsymbol{I}-\boldsymbol{S}^{H}(s) \boldsymbol{S}(s)\right]=0$ are positive for some values of $\omega$, then they are positive for all values of $\omega$. Therefore, $\left[\boldsymbol{I}-\boldsymbol{S}^{H}(s) \boldsymbol{S}(s)\right]$ has only positive eigenvalues, $\boldsymbol{S}(s)$ is bounded real, and consequently, $\boldsymbol{S}(s)$ is passive. This completes the proof.

Illustrative Example: In order to demonstrate the relation between the eigenvalues in Theorem 4.5 and Theorem 4.6, consider a two-port network described by an illustrative state-space system as follows

$$
\begin{aligned}
\boldsymbol{A} & =\left[\begin{array}{cccc}
-50 & 0 & 0 & 0 \\
0 & -30 & 0 & 0 \\
0 & 0 & -30 & 0 \\
0 & 0 & 0 & -50
\end{array}\right], \quad \boldsymbol{B}=\left[\begin{array}{cc}
1 & 0 \\
0 & 1 \\
1 & 0 \\
0 & 1
\end{array}\right], \quad \boldsymbol{C}_{1}=\left[\begin{array}{cccc}
10 & 0 & 0 & 0 \\
0 & 0 & 0 & 10
\end{array}\right] \\
\boldsymbol{C}_{2} & =\left[\begin{array}{llll}
0 & 2 & 0 & 0 \\
0 & 0 & 2 & 0
\end{array}\right], \quad \boldsymbol{D}=\left[\begin{array}{cc}
0.74 & 0 \\
0 & 0.74
\end{array}\right], \quad \tau_{1}=0 \mathrm{~ns}, \quad \tau_{2}=1 \mathrm{~ns} .
\end{aligned}
$$

The eigenvalues at a specific frequency $(0.1905 \mathrm{GHz})$ for $\boldsymbol{H}(j \omega)$ and $\mathcal{M}(j \omega)$ were calculated and are shown in Table 4.1. It is clear from the table that the eigenvalues $\mathcal{M}(j \omega)$ are identical to a subset of eigenvalues in the full-size matrix $\boldsymbol{H}(j \omega)$. It can also be seen that the full-size matrix contains additional eigenvalues that are simply equal to \pm eigenvalues of $\boldsymbol{A}$ (i.e. poles of the macromodel). It should be noted that since the poles in the macromodel are selected such that they are never pure 
TABLE 4.1: A comparison of eigenvalues from the full-size and half-size FDGEP at $0.1905 \mathrm{GHz}$.

\begin{tabular}{|c|c|}
\hline $\begin{array}{c}\text { Full-Size FDGEP } \\
(16 \times 16 \text { Hamiltonian })\end{array}$ & $\begin{array}{c}\text { Half-Size FDGEP } \\
(8 \times 8 \text { Hamiltonian })\end{array}$ \\
\hline \hline $35.2586-6.6584 \mathrm{j}$ & $35.2586-6.6584 \mathrm{j}$ \\
\hline$-35.2586-6.6584 \mathrm{j}$ & $-35.2586-6.6584 \mathrm{j}$ \\
\hline$-22.6977+9.7037 \mathrm{j}$ & $-22.6977+9.7037 \mathrm{j}$ \\
\hline $22.6977+9.7037 \mathrm{j}$ & $22.6977+9.7037 \mathrm{j}$ \\
\hline $35.8330+4.2602 \mathrm{j}$ & $35.8330+4.2602 \mathrm{j}$ \\
\hline$-35.8330+4.2602 \mathrm{j}$ & $-35.8330+4.2602 \mathrm{j}$ \\
\hline $14.1190-7.3055 \mathrm{j}$ & $14.1190-7.3055 \mathrm{j}$ \\
\hline$-14.1190-7.3055 \mathrm{j}$ & $-14.1190-7.3055 \mathrm{j}$ \\
\hline 50 & - \\
\hline 50 & - \\
\hline-50 & - \\
\hline-50 & - \\
\hline 30 & - \\
\hline 30 & - \\
\hline-30 & - \\
\hline-30 & \\
\hline
\end{tabular}

imaginary values, they do not lead to any passivity violations and can be removed from consideration without effecting the passivity verification results. This is essentially exploited in the approach based on the half-size Hamiltonian matrix to avoid redundant computations. This result is summarized as a corollary to Theorem 4.6.

Corollary 4.7 (for Theorem 4.6): The eigenvalues of the FDGEP described by (4.12) contain all the eigenvalues of the FDGEP in (4.25). In addition, the only other eigenvalues in (4.12) correspond to \pm the poles of the network (i.e. \pm the eigenvalues of $\boldsymbol{A})$.

Proof of Corollary 4.7: In order to see the relation between the full and half-size 
FDGEPs, (4.12) is first re-formulated. Note that $(4.22 \mathrm{a})$ plus $(4.22 \mathrm{c})$ gives

$$
\begin{aligned}
s_{0}(\boldsymbol{\delta}+\boldsymbol{\alpha})= & -\boldsymbol{A}^{T}(\boldsymbol{\delta}+\boldsymbol{\alpha})-\hat{\boldsymbol{C}}^{T}\left(s_{0}^{*}\right) \boldsymbol{D} \boldsymbol{R} \boldsymbol{B}^{T}(\boldsymbol{\delta}+\boldsymbol{\alpha})-\hat{\boldsymbol{C}}^{T}\left(s_{0}^{*}\right) \boldsymbol{D} \boldsymbol{R} \boldsymbol{D}^{T} \hat{\boldsymbol{C}}\left(s_{0}\right) \boldsymbol{\gamma} \\
& -\hat{\boldsymbol{C}}^{T}\left(s_{0}^{*}\right) \hat{\boldsymbol{C}}\left(s_{0}\right) \boldsymbol{\theta}
\end{aligned}
$$

In addition, using (4.22b) and (4.20) gives

$$
s_{0}(\boldsymbol{\gamma}-\boldsymbol{\theta})=\boldsymbol{A}(\boldsymbol{\gamma}-\boldsymbol{\theta})
$$

Next, define the variables $\varphi=\gamma-\boldsymbol{\theta}$ and $\boldsymbol{\beta}=\boldsymbol{\delta}+\boldsymbol{\alpha}$. Then, note that from the definitions in (4.19b) and (4.20) that $\boldsymbol{\gamma}=\boldsymbol{\theta}$. Combining these definitions in the above equations along with (4.22b) and (4.22c) gives the following system

$$
\begin{aligned}
& s_{0} \boldsymbol{\beta}=-\boldsymbol{A}^{T} \boldsymbol{\beta}-\hat{\boldsymbol{C}}^{T}\left(s_{0}^{*}\right) \boldsymbol{D} \boldsymbol{R} \boldsymbol{B}^{T} \boldsymbol{\beta}-\hat{\boldsymbol{C}}^{T}\left(s_{0}^{*}\right)\left(\boldsymbol{D} \boldsymbol{R} \boldsymbol{D}^{T}+\boldsymbol{I}\right) \hat{\boldsymbol{C}}\left(s_{0}\right) \boldsymbol{\gamma} \\
& s_{0} \boldsymbol{\varphi}=\boldsymbol{A} \boldsymbol{\varphi} \\
& s_{0} \boldsymbol{\gamma}=\boldsymbol{A} \boldsymbol{\gamma}+\boldsymbol{B} \boldsymbol{R} \boldsymbol{B}^{T} \boldsymbol{\beta}+\boldsymbol{B} \boldsymbol{R} \boldsymbol{D}^{T} \hat{\boldsymbol{C}}\left(s_{0}\right) \boldsymbol{\gamma} \\
& s_{0} \boldsymbol{\alpha}=-\boldsymbol{A}^{T} \boldsymbol{\alpha}-\hat{\boldsymbol{C}}^{T}\left(s_{0}^{*}\right) \hat{\boldsymbol{C}}\left(s_{0}\right) \boldsymbol{\gamma} .
\end{aligned}
$$

Writing this in matrix form and using $\hat{\boldsymbol{R}}=\left[\boldsymbol{D} \boldsymbol{R} \boldsymbol{D}^{T}+\boldsymbol{I}\right]=\left(\boldsymbol{I}-\boldsymbol{D} \boldsymbol{D}^{T}\right)^{-1}$ gives the equivalent formulation for (4.12) as

$$
\begin{aligned}
& s \zeta=H(s) \zeta, \quad \zeta=\left[\begin{array}{llll}
\gamma^{T} & \beta^{T} & \varphi^{T} & \alpha^{T}
\end{array}\right]^{T} \\
& \boldsymbol{H}(s)=\mathcal{V}+\sum_{m=0}^{M} \hat{\boldsymbol{W}}_{m} e^{-s \tau_{m}}+\sum_{m=0}^{M} \overline{\boldsymbol{W}}_{m} e^{s \tau_{m}}+\sum_{m, n=0}^{M} \boldsymbol{W}_{m n} e^{s\left(\tau_{m}-\tau_{n}\right)}
\end{aligned}
$$


where

$$
\begin{aligned}
& \mathcal{V}=\left[\begin{array}{cccc}
\boldsymbol{A} & \boldsymbol{B} \boldsymbol{R} \boldsymbol{B}^{T} & \mathbf{0} & \mathbf{0} \\
\mathbf{0} & -\boldsymbol{A}^{T} & \mathbf{0} & \mathbf{0} \\
\mathbf{0} & \mathbf{0} & \boldsymbol{A} & \mathbf{0} \\
\mathbf{0} & \mathbf{0} & \mathbf{0} & -\boldsymbol{A}^{T}
\end{array}\right], \quad \hat{\boldsymbol{W}}_{m}=\left[\begin{array}{cccc}
\boldsymbol{B} \boldsymbol{R} \boldsymbol{D}^{T} \boldsymbol{C}_{m} & \mathbf{0} & \mathbf{0} & \mathbf{0} \\
\mathbf{0} & \mathbf{0} & \mathbf{0} & \mathbf{0} \\
\mathbf{0} & \mathbf{0} & \mathbf{0} & \mathbf{0} \\
\mathbf{0} & \mathbf{0} & \mathbf{0} & \mathbf{0}
\end{array}\right]
\end{aligned}
$$

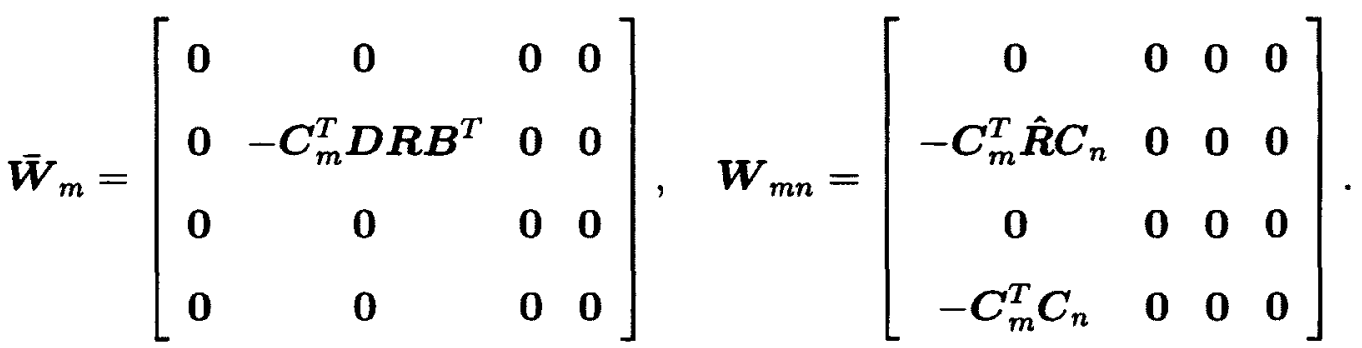

Next, it will be shown through simple manipulations that the bottom left term in $\boldsymbol{W}_{m n},-\boldsymbol{C}_{m}^{T} \boldsymbol{C}_{n}$, does not contribute to the eigenvalues of $\boldsymbol{H}(s)$. It will also become clear that the eigenvalues of (4.48) contain the eigenvalues of (4.26), and that the remaining eigenvalues of $\boldsymbol{H}(s)$ in (4.48) are the eigenvalues of $\boldsymbol{A}$ and $-\boldsymbol{A}^{T}$.

Note that $\mathcal{M}(s)$ from (4.26) can be written in the form

$$
\mathcal{M}(s)=\left[\begin{array}{ll}
\boldsymbol{\Upsilon}_{a} & \Upsilon_{\boldsymbol{b}} \\
\boldsymbol{\Upsilon}_{c} & \boldsymbol{\Upsilon}_{\boldsymbol{d}}
\end{array}\right]
$$

where $\boldsymbol{\Upsilon}_{\boldsymbol{a}}, \boldsymbol{\Upsilon}_{\boldsymbol{b}}, \boldsymbol{\Upsilon}_{\boldsymbol{c}}$, and $\boldsymbol{\Upsilon}_{\boldsymbol{d}}$ represent the matrix functions in (4.26) (for example, $\left.\boldsymbol{\Upsilon}_{\boldsymbol{a}}=\boldsymbol{A}+\sum_{n=0}^{M} \boldsymbol{B} \boldsymbol{R} \boldsymbol{D}^{T} \boldsymbol{C}_{n} e^{-s \tau_{n}}\right)$. Let $\boldsymbol{\Lambda}_{\boldsymbol{M}}$ be the matrix of eigenvalues of $\mathcal{M}(s)$. Recall that the eigenvalues of a matrix occur when its determinant or characteristic polynomial become equal to zero. For $\mathcal{M}(s)$, the characteristic matrix polynomial 
defining the eigenvalues is given by

$$
\mathcal{P}(\mathcal{M})=\left(\boldsymbol{\Lambda}_{M}-\boldsymbol{\Upsilon}_{a}\right)\left(\boldsymbol{\Lambda}_{M}-\boldsymbol{\Upsilon}_{d}\right)-\boldsymbol{\Upsilon}_{b} \boldsymbol{\Upsilon}_{c}=\mathbf{0}
$$

Next, $\boldsymbol{H}(s)$ from (4.48) is written in the form

$$
H(s)=\left[\begin{array}{cccc}
\Upsilon_{a} & \Upsilon_{b} & 0 & 0 \\
\Upsilon_{c} & \Upsilon_{d} & 0 & 0 \\
0 & 0 & A & 0 \\
\Upsilon_{e} & 0 & 0 & -A^{T}
\end{array}\right]
$$

where $\Upsilon_{e}=\sum_{m, n=0}^{M} C_{m}^{T} C_{n} e^{s\left(\tau_{m}-\tau_{n}\right)}$. Let $\Lambda_{\boldsymbol{H}}$ be the eigenvalues for the full-size FDGEP, $\boldsymbol{H}(s)$. The characteristic matrix polynomial defining these eigenvalues is given by

$$
\begin{aligned}
& \mathcal{P}(\boldsymbol{H})=\left|\begin{array}{cccc}
\Lambda_{H}-\Upsilon_{a} & \boldsymbol{\Upsilon}_{b} & 0 & 0 \\
\boldsymbol{\Upsilon}_{c} & \Lambda_{H}-\Upsilon_{d} & 0 & 0 \\
0 & 0 & \Lambda_{H}-\boldsymbol{A} & 0 \\
\Upsilon_{e} & 0 & 0 & \Lambda_{H}+A^{T}
\end{array}\right| \\
& =\left(\boldsymbol{\Lambda}_{H}-\boldsymbol{\Upsilon}_{a}\right)\left|\begin{array}{ccc}
\Lambda_{H}-\boldsymbol{\Upsilon}_{d} & 0 & 0 \\
0 & \Lambda_{H}-\boldsymbol{A} & 0 \\
0 & 0 & \Lambda_{H}+A^{T}
\end{array}\right|-\Upsilon_{b}\left|\begin{array}{lll}
\Upsilon_{c} & 0 & 0 \\
0 & \Lambda_{H}-A & 0 \\
\Upsilon_{e} & 0 & \Lambda_{H}+A^{T}
\end{array}\right|
\end{aligned}
$$

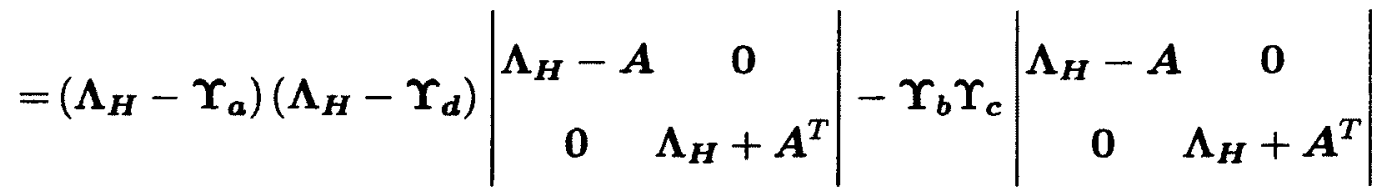

$$
\begin{aligned}
& =\left\{\left(\boldsymbol{\Lambda}_{\boldsymbol{H}}-\boldsymbol{\Upsilon}_{\mathbf{a}}\right)\left(\boldsymbol{\Lambda}_{\boldsymbol{H}}-\boldsymbol{\Upsilon}_{\mathbf{d}}\right)-\boldsymbol{\Upsilon}_{\mathbf{b}} \boldsymbol{\Upsilon}_{\mathbf{c}}\right\} \cdot\left\{\left(\boldsymbol{\Lambda}_{\boldsymbol{H}}-\boldsymbol{A}\right)\left(\boldsymbol{\Lambda}_{\boldsymbol{H}}+\boldsymbol{A}^{T}\right)\right\}=\mathbf{0} \text {. }
\end{aligned}
$$


It is clear that the zeros of the characteristic matrix polynomial (4.53) contain the zeros of (4.51), while the remaining zeros correspond to the eigenvalues of $\boldsymbol{A}$ and $-\boldsymbol{A}^{T}$ (which are nothing but the poles of the macromodel). Since the zeros of (4.53) and (4.51) correspond to the eigenvalues of $\boldsymbol{H}(s)$ and $\mathcal{M}(s)$, respectively, this completes the proof.

Computational Complexity: For an $n \times n$ matrix, the number of floating point operations of typical eigenvalue calculation is $O\left(n^{3}\right)$ [77]. Consequently, solving the half-size FDGEP of Theorem 4.6 over the full-size FDGEP of Theorem 4.5 will typically yield a speedup by a factor of 8 .

\subsection{Identification of Passivity Verification Regions}

It is to be noted that Theorem 4.6 specifies the necessary and sufficient conditions for the DRF-based macromodel to become nonpassive. Currently the only relation between the eigenvalues of the FDGEP described by (4.25) and the eigenvalues of $\left[\boldsymbol{I}-\boldsymbol{S}^{H}\left(s_{0}\right) \boldsymbol{S}\left(s_{0}\right)\right]$ is that, the generalized eigenvalues of (4.25) (or (4.12)) become purely imaginary when those of $\left[\boldsymbol{I}-\boldsymbol{S}^{H}\left(s_{0}\right) \boldsymbol{S}\left(s_{0}\right)\right]$ change their signs. However, for the purpose of passivity compensation, it is also necessary to locate the regions of passivity violation in the DRF macromodel. For this purpose, the following theorem is developed.

Theorem 4.8: The eigenvalue(s) of $\left[\boldsymbol{I}-\boldsymbol{S}^{H}\left(s_{0}\right) \boldsymbol{S}\left(s_{0}\right)\right]$ for a nonpassive DRF macromodel vanish at the point(s) $s_{0}=j \omega$ on the imaginary axis that satisfy (4.25).

Proof of Theorem 4.8: From Theorem 4.6, if $s_{0}$ is an imaginary value satisfying (4.25), then the matrix $\left[\boldsymbol{I}-\boldsymbol{S}^{H}\left(s_{0}\right) \boldsymbol{S}\left(s_{0}\right)\right]$ is singular and the system becomes 
nonpassive.

It can be concluded from Theorem 4.6 and Theorem 4.8 that the regions of passivity violation in the DRF macromodel are delimited by the purely imaginary eigenvalues that satisfy (4.25).

Several techniques for solving quasi-periodic FDGEPs are available in the literature [50]-[52]. If a passivity violation is found, then it is necessary to apply a passivity compensation algorithm to iteratively perturb the macromodel until it becomes passive. For this purpose, in the next section a new passivity compensation algorithm is developed for DRF-based macromodels obtained from tabulated scattering parameter data.

\subsection{Proposed Passivity Compensation Algorithm}

Consider a macromodel described by the Laplace-domain transfer function given in (4.11). For the purpose of illustration, assume that the nonpassive macromodel contains one passivity violation. Let $\omega_{1}$ and $\omega_{2}$ be the upper and lower bounds of the passivity violation region and let $\lambda_{\min }$ be the minimum eigenvalue of $\left[\boldsymbol{I}-\boldsymbol{S}(s)^{H} \boldsymbol{S}(s)\right]$ occurring at $\omega=\omega_{\lambda}$. The goal of passivity compensation is to perturb the scattering matrix, $\boldsymbol{S}(s)$, until $\lambda_{\min }$ becomes positive and the final macromodel is passive. This is achieved by perturbing each of the residue matrices, $\left\{\boldsymbol{C}_{m}\right\}, m \in(0,1, \ldots, M)$, to obtain

$$
\hat{C}_{m}=C_{m}+\Delta C_{m}
$$


as the new residue matrices. This will yield a new macromodel with a scattering matrix given by

$$
\hat{\boldsymbol{S}}(j \omega)=\boldsymbol{S}(j \omega)+\sum_{m=0}^{M} \Delta \boldsymbol{S}_{m}(j \omega)
$$

where $\Delta \boldsymbol{S}_{m}(j \omega)$ is the perturbing effect of $\Delta \boldsymbol{C}_{m}$.

Recall that, for a macromodel to be passive, it must satisfy the following condition

$$
\boldsymbol{I}-\hat{\boldsymbol{S}}^{H}(j \omega) \hat{\boldsymbol{S}}(j \omega) \geq 0
$$

Using the definition of (4.55) for $\hat{\boldsymbol{S}}$ (and neglecting the $j \omega$ ) gives

$$
\boldsymbol{I}-\boldsymbol{S}^{H} \boldsymbol{S}-\sum_{m=0}^{M}\left(\Delta \boldsymbol{S}_{m}^{H} \boldsymbol{S}-\boldsymbol{S}^{H} \Delta \boldsymbol{S}_{m}\right)-\sum_{m, n=0}^{M} \Delta \boldsymbol{S}_{m}^{H} \Delta \boldsymbol{S}_{n} \geq 0
$$

To determine the $\Delta C_{m}$, let $\Delta \lambda$ be the required perturbation to make $\lambda_{\min }$ positive and the macromodel passive. This gives

$$
\Delta \lambda+\lambda_{\min } \geq 0
$$

Then, neglecting the second order terms $\Delta \boldsymbol{S}_{m}^{H} \boldsymbol{\Delta} \boldsymbol{S}_{n}$, first-order perturbation theory [89] can be used to relate $\Delta \lambda$ to each of the $\Delta \boldsymbol{S}_{m}$

$$
\Delta \lambda \approx \frac{\boldsymbol{\vartheta}^{H}\left(-\boldsymbol{S}^{H} \sum_{m=0}^{M} \Delta \boldsymbol{S}_{m}-\sum_{m=0}^{M} \Delta \boldsymbol{S}_{m}^{H} \boldsymbol{S}\right) \boldsymbol{\xi}}{\boldsymbol{\vartheta}^{H} \boldsymbol{\xi}}
$$

where $\boldsymbol{\vartheta}$ and $\boldsymbol{\xi}$ are the left and right eigenvectors of $\left[\boldsymbol{I}-\boldsymbol{S}^{H} \boldsymbol{S}\right]$, respectively, at $\omega=\omega_{\lambda}$ corresponding to the eigenvalue $\lambda_{\min }$.

Substituting (4.59) into (4.58) and after some simple mathematical manipulations 
we obtain

$$
\boldsymbol{\Psi} \boldsymbol{x} \geq-\lambda_{\min }
$$

where the matrix $\boldsymbol{\Psi}$ is a function of $\boldsymbol{\vartheta}, \boldsymbol{\xi}$, and $\boldsymbol{S}(j \omega)$ at $\omega=\omega_{\lambda}$ and $\boldsymbol{x}$ is a column vector corresponding to the entries in the matrices $\left\{\Delta C_{m}\right\}$.

In order to minimize the error introduced in the system we minimize the Frobenius norm of the total perturbation, $\Delta \boldsymbol{S}$, and we show that this in turn minimizes the upper bound on the $L_{2}$-norm of $\Delta S$. To illustrate this, note that the $L_{2}$-norm of $\Delta S$ can be written as follows [53]

$$
\|\Delta\|_{2}^{2}=\left\|\sum_{m=0}^{M} \Delta \boldsymbol{S}_{m}\right\|_{2}^{2} \leq \sum_{m=0}^{M}\left\|\Delta \boldsymbol{S}_{m}\right\|_{2}^{2}=\sum_{m=0}^{M} \int_{-\infty}^{\infty}\left\|\Delta \boldsymbol{S}_{m}\right\|_{F}^{2} d \omega
$$

where $\|\cdot\|_{2}$ and $\|\cdot\|_{F}$ are the $L_{2}$-norm and the Frobenius norm of the argument, respectively. Substituting in $\Delta \boldsymbol{S}_{m}=e^{-j \omega \tau_{m}} \Delta \boldsymbol{C}_{m}(j \omega \boldsymbol{I}-\boldsymbol{A})^{-1} \boldsymbol{B}$ gives

$$
\|\Delta \boldsymbol{S}\|_{2}^{2} \leq \sum_{m=0}^{M} \int_{-\infty}^{\infty}\left\|e^{-j \omega \tau_{m}} \Delta \boldsymbol{C}_{m}(j \omega \boldsymbol{I}-\boldsymbol{A})^{-1} \boldsymbol{B}\right\|_{F}^{2} d \omega=\sum_{m=0}^{M} \operatorname{trace}\left(\Delta \boldsymbol{C}_{m} \boldsymbol{P} \Delta \boldsymbol{C}_{m}^{T}\right)
$$

The matrix $\boldsymbol{P}$ is the controllability Grammian obtained by solving the following Lyapunov equation [91]

$$
\boldsymbol{A P}+\boldsymbol{P} \boldsymbol{A}^{H}+\boldsymbol{B} \boldsymbol{B}^{H}=\mathbf{0} .
$$

Note $\boldsymbol{P}$ is only calculated once since it is independent of each of the $\Delta \boldsymbol{C}_{m}$ and the matrices $\boldsymbol{A}$ and $\boldsymbol{B}$ are constant. It is clear from (4.62) that minimizing the sum of the traces $\Delta C_{m} P \Delta C_{m}^{T}$ ensures that the error introduced in the time and frequencydomain responses is kept as small as possible. With the addition of error control, the passivity enforcement scheme for nonpassive DRF macromodels amounts to solving 
the following problem for the $\left\{\Delta C_{m}\right\}$ :

$$
\min _{\left\{\Delta C_{m}\right\}} \sum_{m=0}^{M} \operatorname{trace}\left(\Delta \boldsymbol{C}_{m} P \Delta \boldsymbol{C}_{m}^{T}\right), \quad \text { subject to }(4.60)
$$

For the above discussion, it was assumed that passivity was enforced in a single step. However, since we are using techniques based on first-order perturbation theory, in practice only a relatively small amount of passivity compensation can be done at once. Consequently, the above method is applied iteratively, where after each iteration, passivity verification is repeated to check for any additional passivity violations that have been introduced. If additional violations are found, then they are compensated in the subsequent iteration. While the derivations in this section were developed for a single passivity violation point, they are easily applied to multiple frequency points at once.

\subsection{Numerical Results}

In this section, three industrial examples are presented to demonstrate the accuracy and efficiency of the proposed method for passivity verification of delayed rational function based macromodels. In addition, the examples demonstrate the improved efficiency of the proposed half-size Hamiltonian based FDGEP formulation over the full-size FDGEP formulation. All the examples are run on an Intel $i 7-9202.67 \mathrm{GHz}$ processor with $12 \mathrm{~Gb}$ of RAM. 


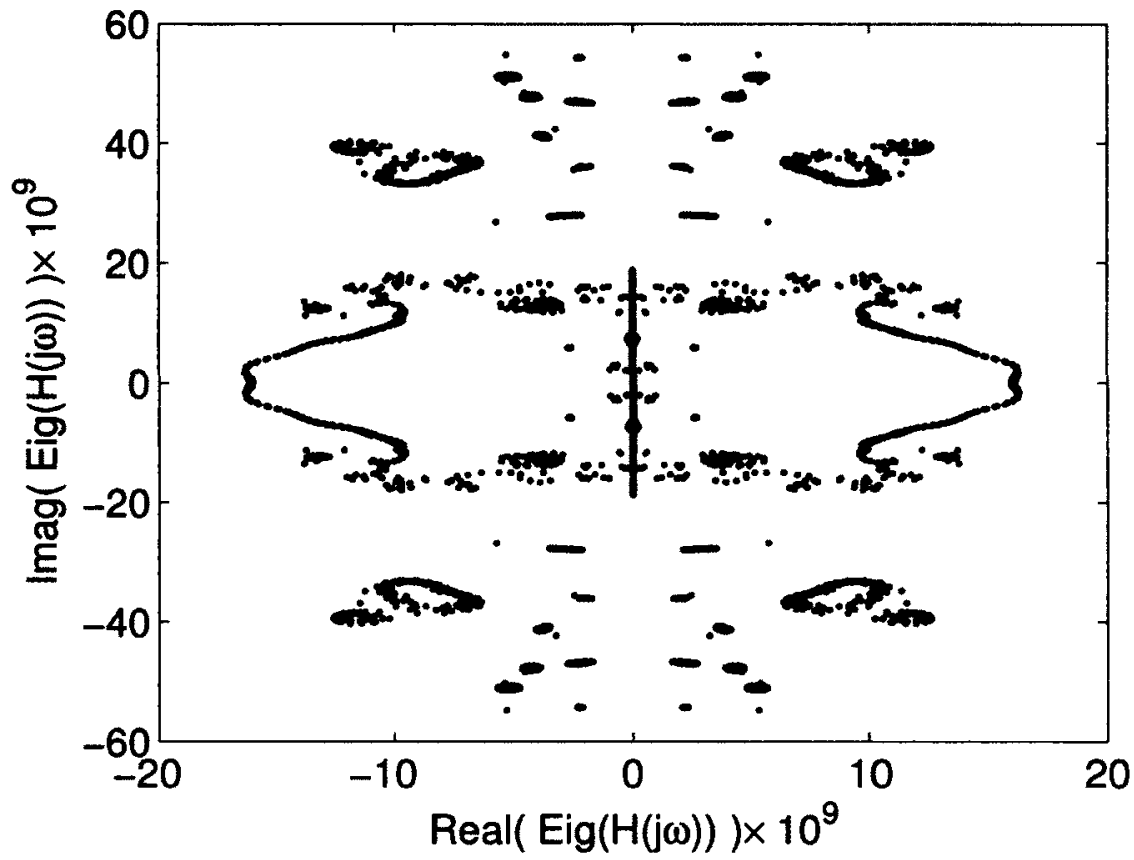

FIGURE 4.2: Eigenvalue spectrum of the full-size FDGEP for example 1.

\subsubsection{Example 1}

In this example, the proposed algorithm was tested on a practical two-port network (a cascade of a microstrip and a coaxial-to-microstrip coupler) described by tabulated scattering parameters up to $9 \mathrm{GHz}$ (provided by CST Gmbh, Germany). A delayed rational function based macromodel was obtained by approximating the data using the delayed vector fitting algorithm [29]. A total of 6 delay terms and 40 poles were required (each parameter was fit separately).

Next, we discuss the passivity verification based on the proposed reduced search region algorithms of Section 4.3. Using the proposed search reduction algorithms based on the FDGEP and full-size Hamiltonian formulation, and efficient FDGEP techniques [50]-[52], the maximum positive imaginary eigenvalue was found to be $18.76 \times 10^{9} \mathrm{rad} / \mathrm{s}$ (which corresponds to the maximum frequency for passivity violation 
search (i.e. $f_{\max }=18.76 \times 10^{9} /(2 \pi)=2.99 \mathrm{GHz}$ )). The corresponding eigenvalue spectrum (on the imaginary axis) is given in Fig. 4.2. Next, using Theorem 4.8, the nonpassive regions are determined to be $[0.5877,1.0712] \mathrm{GHz}$ and $[2.2531,2.3576]$ GHz.

The above results are cross-verified by plotting a fine frequency-sweep of the magnitude spectrum of the scattering matrix and are given in Fig. 4.3(a) (shown in solid line). Also, an expanded view of the passivity violations between 0 and $3 \mathrm{GHz}$ is shown in Fig. 4.3(b). As seen from Fig. 4.3(b), the above theoretically computed passivity violation regions match with the frequency-sweep based analysis, thus validating the proposed theory.

Next, the experiments are repeated with the proposed half-size Hamiltonian formulation of Section 4.4, and identical results are obtained (i.e. passivity violation regions of $[0.5877,1.0712] \mathrm{GHz}$ and $[2.2531,2.3576] \mathrm{GHz})$, thus validating the accuracy of the half-Hamiltonian based approach. Table 4.2 gives a comparison of the CPU performance for obtaining an upper bound on the passivity verification region using the both the full-size and half-size FDGEP.

Next, the proposed first-order based passivity compensation process was applied and the macromodel was corrected for passivity violations. As seen from the scattering matrix norm frequency-sweep in Fig. 4.3(a) and 4.3(b) (given in dotted lines), the passivity violations were compensated in the new macromodel. Fig. 4.4 shows a sample accuracy comparison and as seen, the compensated macromodel is in good agreement with the original response. 


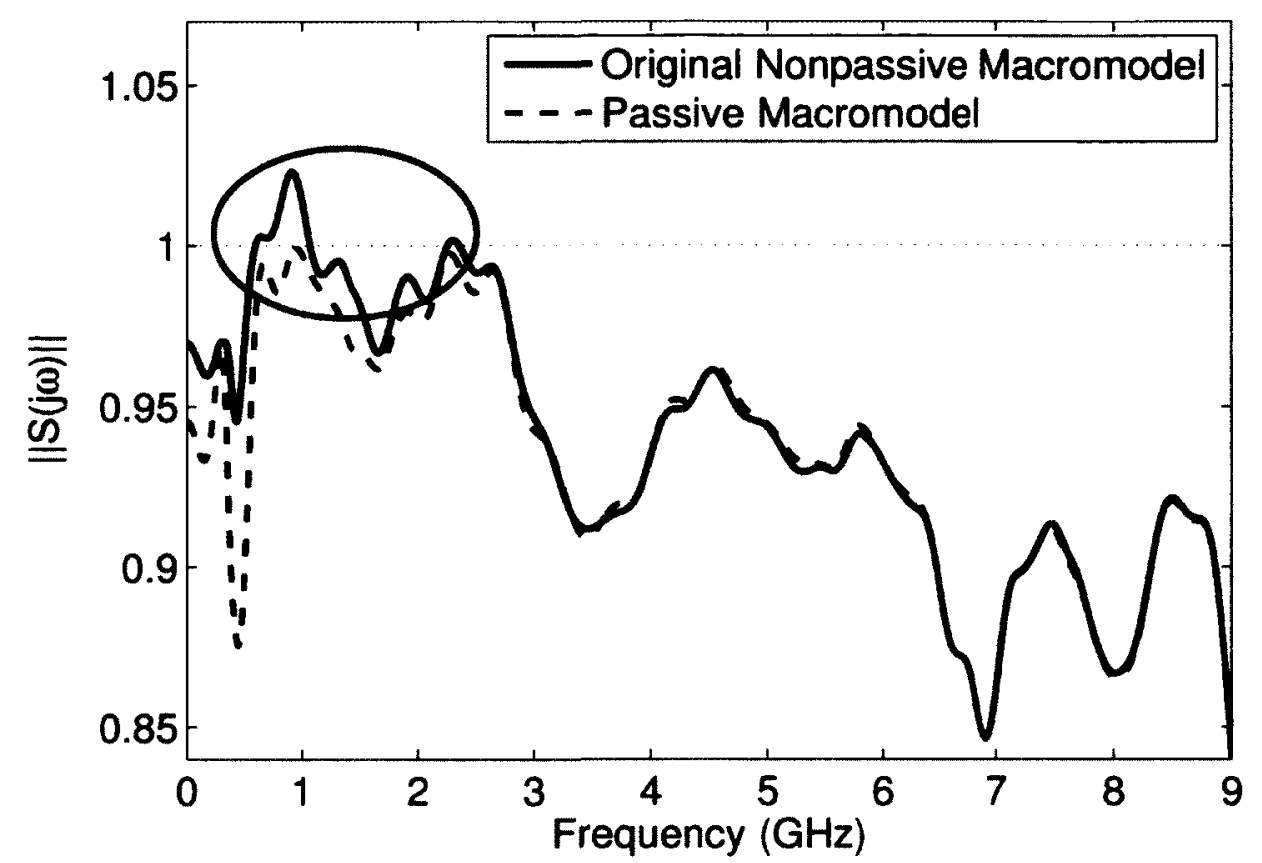

(a) Behavior of the norm of the scattering parameters.

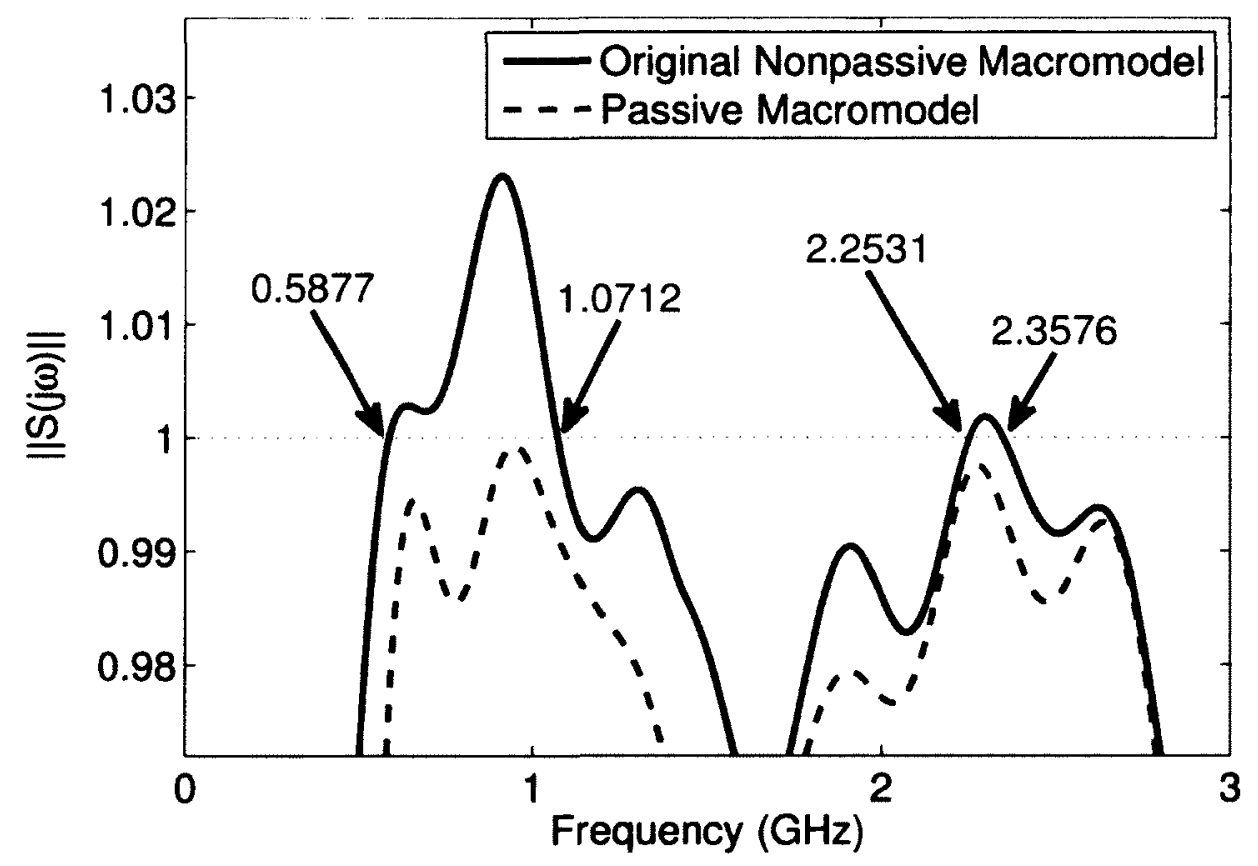

(b) An expanded view of the circled region in Fig. 4.3(a).

FIGURE 4.3: Passivity violations of the DRFs for example 1. 
TABLE 4.2: A comparison of CPU time for obtaining an upper bound from the full-size and half-size FDGEP.

\begin{tabular}{|c|c|c|c|}
\hline & Full-Size FDGEP (sec) & Half-Size FDGEP (sec) & Speed-up \\
\hline \hline Example 1 & 0.0209 & 0.0078 & 2.7 \\
\hline Example 2 & 0.1266 & 0.0260 & 4.9 \\
\hline Example 3 & 661.4376 & 52.9528 & 12.5 \\
\hline
\end{tabular}

\subsubsection{Example 2}

In this example, the proposed algorithm was tested on a practical four-port microstrip coupler described by tabulated scattering parameters up to $1 \mathrm{GHz}$ (provided by CST Gmbh, Germany). The delayed vector fitting algorithm [29] was applied to the data to obtain a delayed rational function based macromodel. The macromodel required a total of 3 delay terms and 88 poles (each parameter was fit separately).

Next, the proposed passivity verification based on the reduced search region algorithms of Section 4.3 is applied. Using the proposed search reduction algorithms based on the FDGEP and full-size Hamiltonian formulation, the maximum positive imaginary eigenvalue was found to be $7.96 \times 10^{9} \mathrm{rad} / \mathrm{s}$ (which corresponds to the maximum frequency for passivity violation search (i.e. $f_{\max }=7.96 \times 10^{9} /(2 \pi)=1.27 \mathrm{GHz}$ )) . The corresponding eigenvalue spectrum for the full-size FDGEP is given in Fig. 4.5. Next, a single region of passivity violations is found on the interval $[0.0510,0.0513]$ GHz (using Theorem 4.8).

In order to cross-verify the above results, a fine frequency-sweep of the magnitude spectrum of the scattering matrix is given in Fig. 4.6(a) (shown in solid line). An expanded view of the spectrum between 0.0508 and $0.0517 \mathrm{GHz}$ is shown in Fig. 4.6(b). As seen from Fig. 4.6(b), the above theoretically computed passivity violation region matches accurately with the frequency-sweep based analysis, thus validating the proposed theory. 


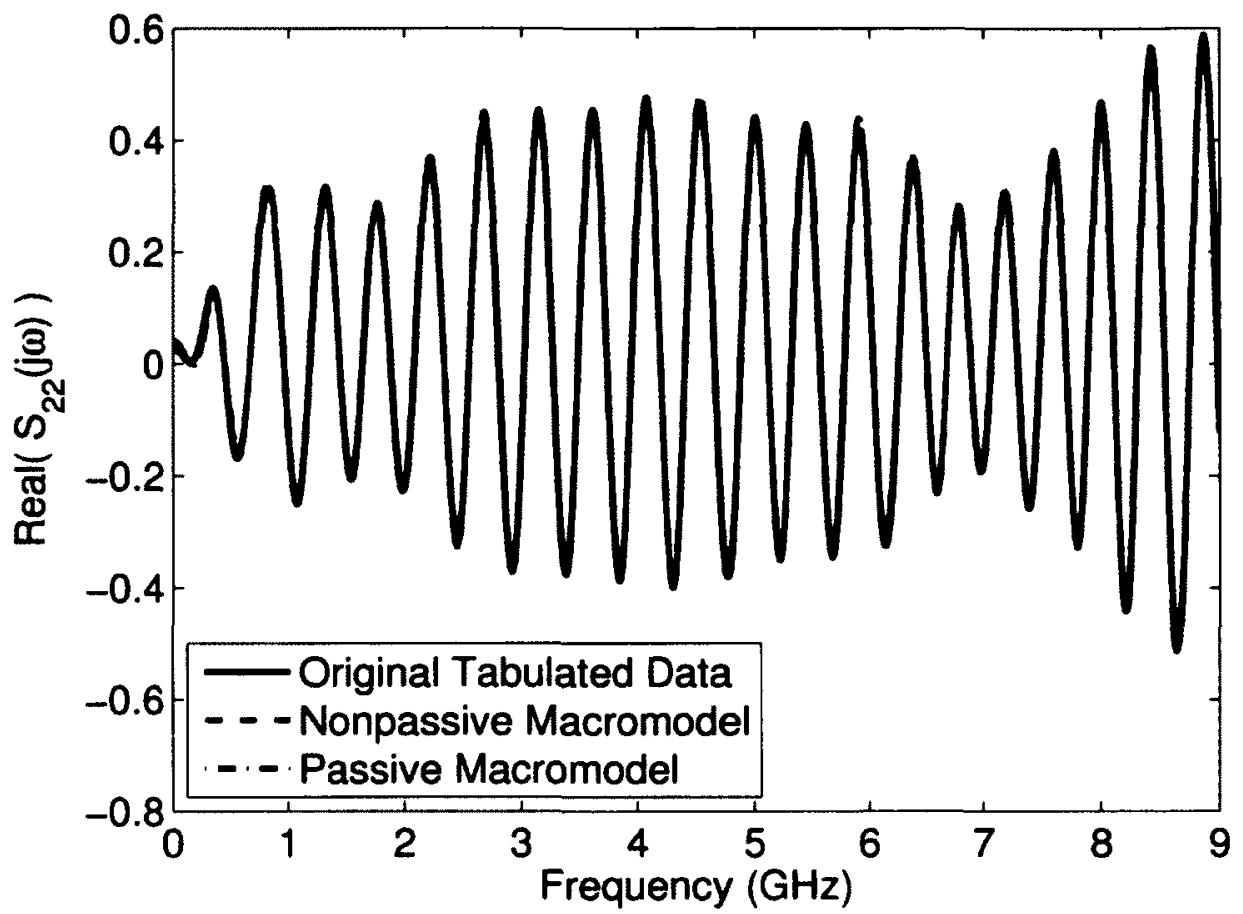

FIGURE 4.4: Accuracy comparison of the scattering parameters for example 1 (the Original Tabulated Data, Nonpassive Macromodel, and Passive Macromodel are all overlapping).

Next, the above passivity verification process is repeated with the proposed halfsize FDGEP developed in Section 4.4. The results obtained were identical to the full-size FDGEP (i.e. a single passivity violation region of $[0.0510,0.0513] \mathrm{GHz}$ was found), thus validating the accuracy of the half-size Hamiltonian based approach. Table 4.2 gives a comparison of the CPU performance for obtaining an upper bound on the passivity verification region using the both the full-size and half-size FDGEP.

In order to construct a final, passive macromodel, the proposed first-order passivity compensation technique is applied to the macromodel to correct the passivity violations. As seen from the scattering-matrix norm frequency-sweep in Fig. 4.6(a) and 4.6(b) (given in dotted lines), the new compensated macromodel does not contain any passivity violations. Fig. 4.7 shows a sample accuracy comparison and as seen, 


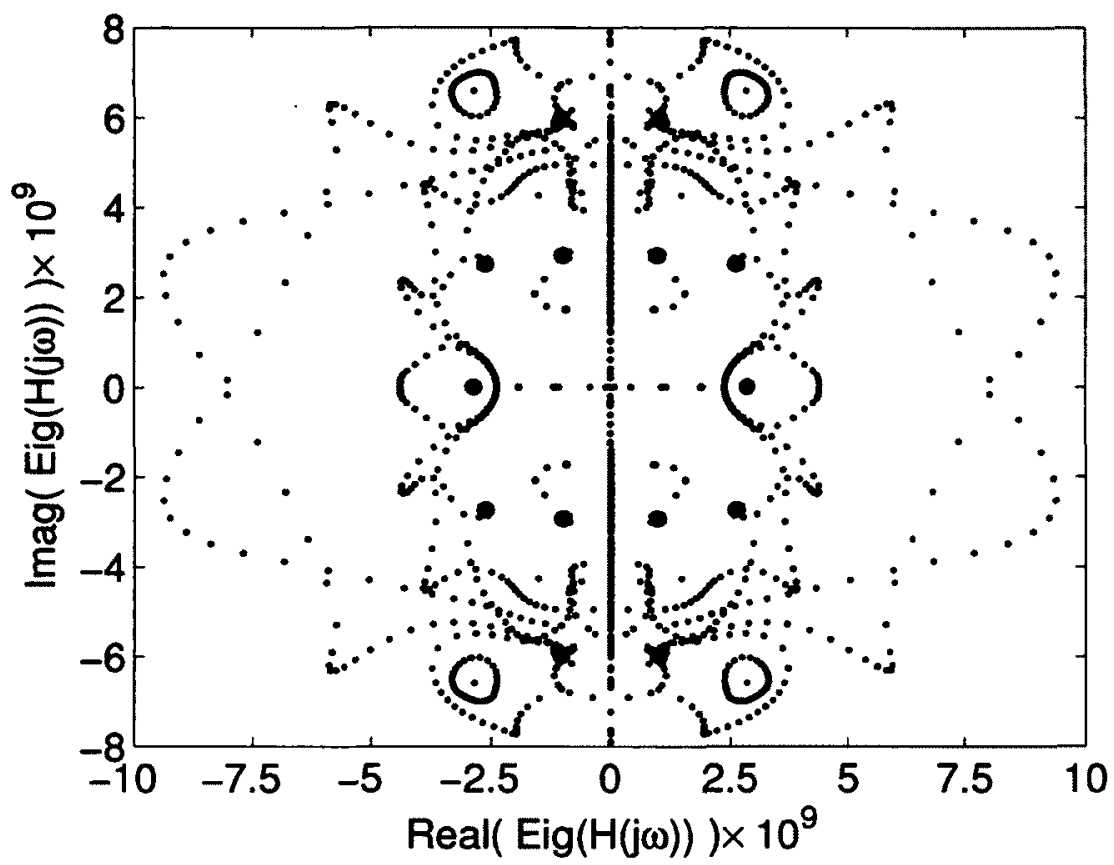

FIGURE 4.5: Eigenvalue spectrum of the full-size FDGEP for example 2.

the compensated macromodel is in good agreement with the original response.

\subsubsection{Example 3}

In this example, the proposed algorithm was tested on an industrial four-port network corresponding to a daughtercard-connector-backplane-connector-daughtercard system illustrated in Fig. 4.8 (provided by Tyco Electronics Inc.). Each connector is the Tyco Z-Pack TINMAN connector. The backplane is a 24 inch transmission line with trace geometry shown in Fig. 4.9. The device is characterized by measured scattering parameters given up to $8 \mathrm{GHz}$. A delayed rational function based macromodel was obtained by approximating the data using the delayed vector fitting algorithm [29]. A total of 7 delay terms and 1184 poles were required (each parameter was fit separately). A sample of the scattering parameters is shown in Fig. 4.10. 

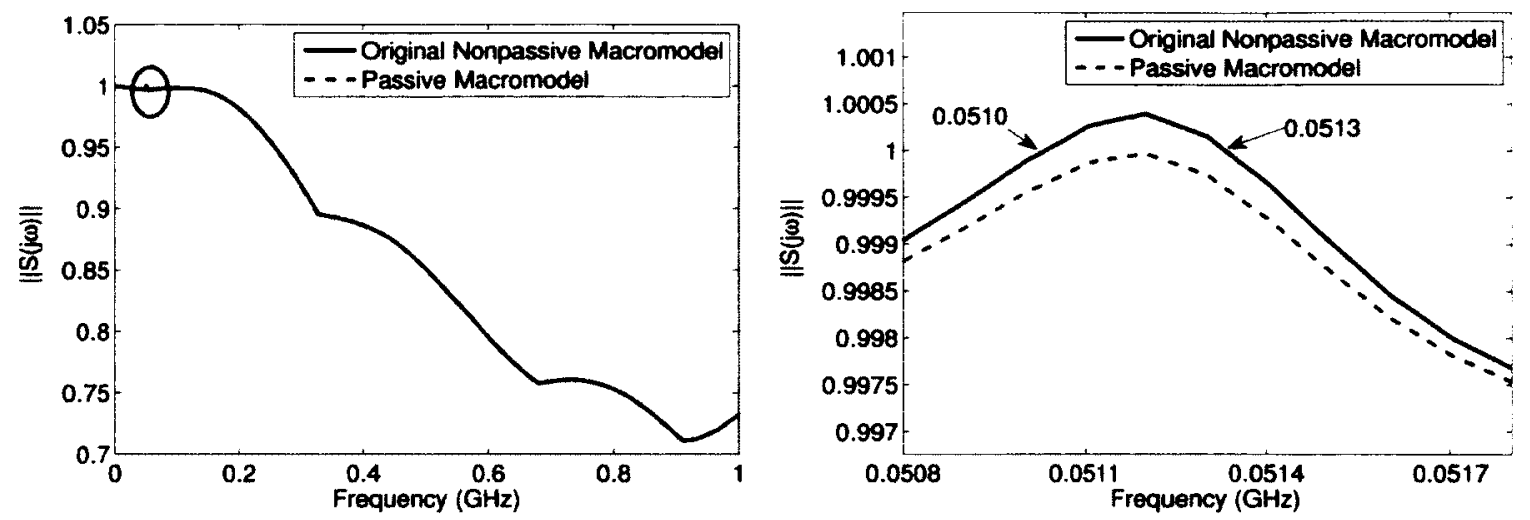

(a) Behavior of the norm of the scattering pa- (b) An expanded view of the circled region in Fig. rameters (the Original and Passive Macromodel 4.6(a). are overlapping).

FIGURE 4.6: Passivity violations of the DRFs for example 2.

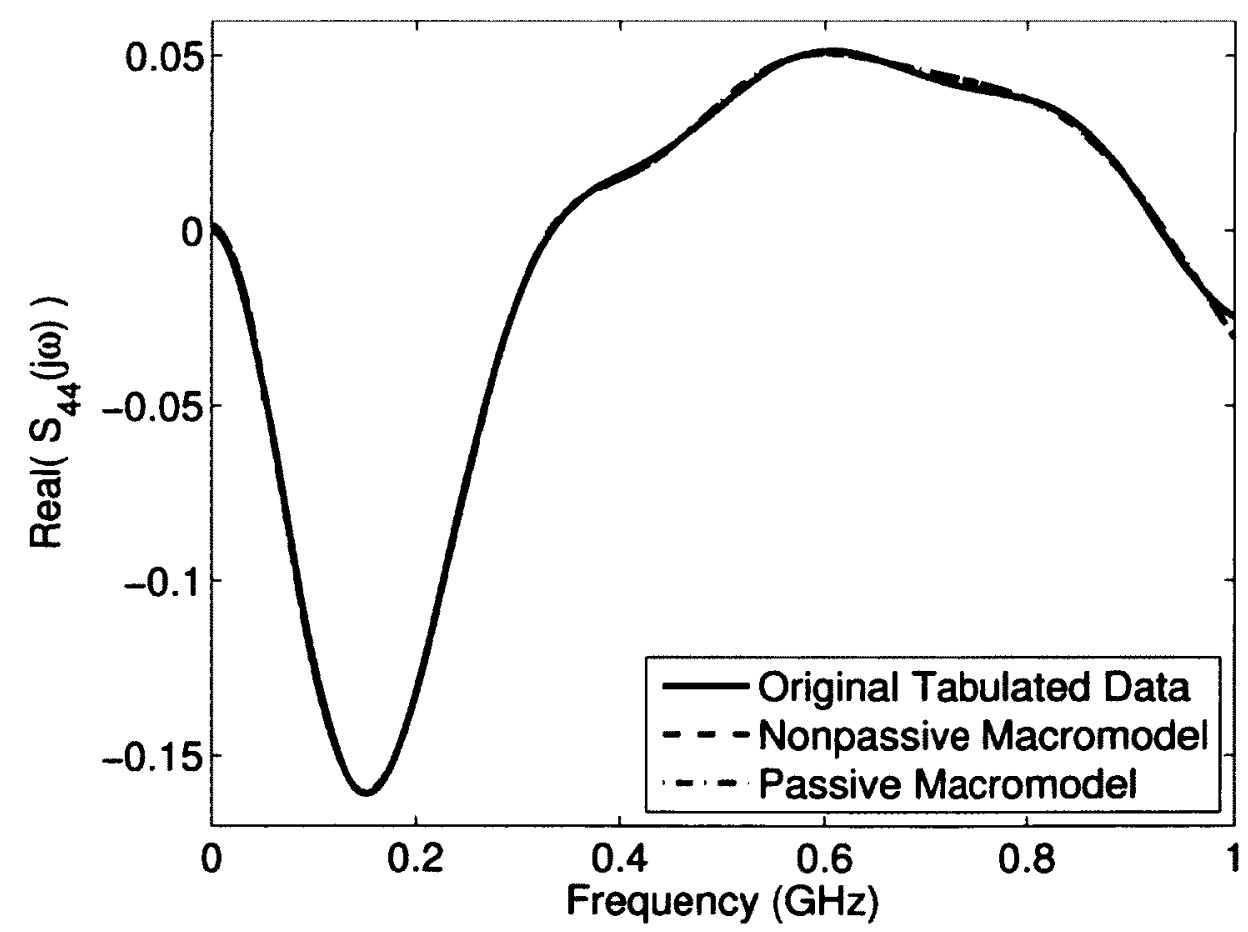

FIGURE 4.7: Comparison of scattering parameters for example 2 (the Original Tabulated Data, Nonpassive Macromodel, and Passive Macromodel are all overlapping). 


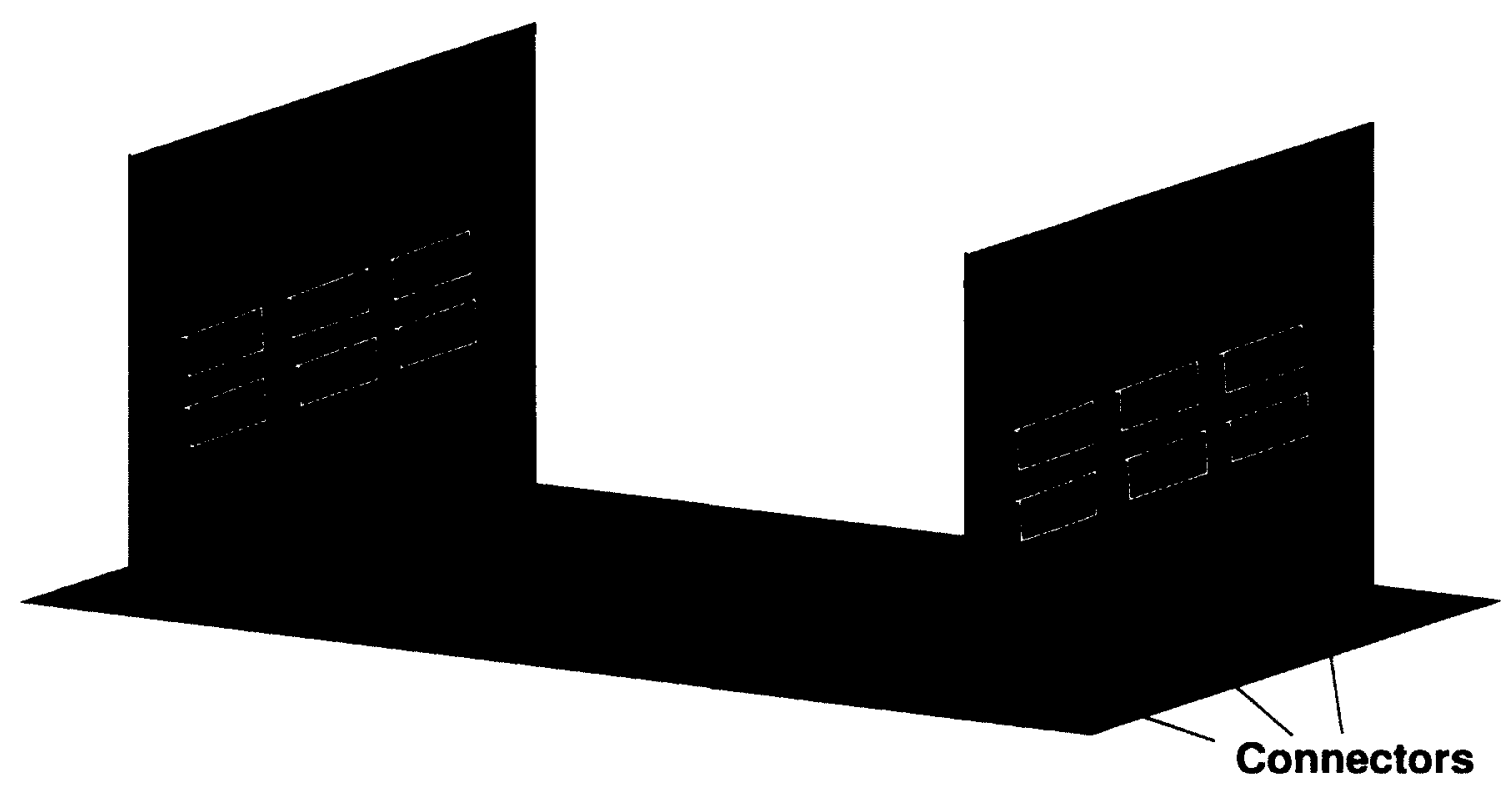

FIGURE 4.8: Electrical system for example 3 .

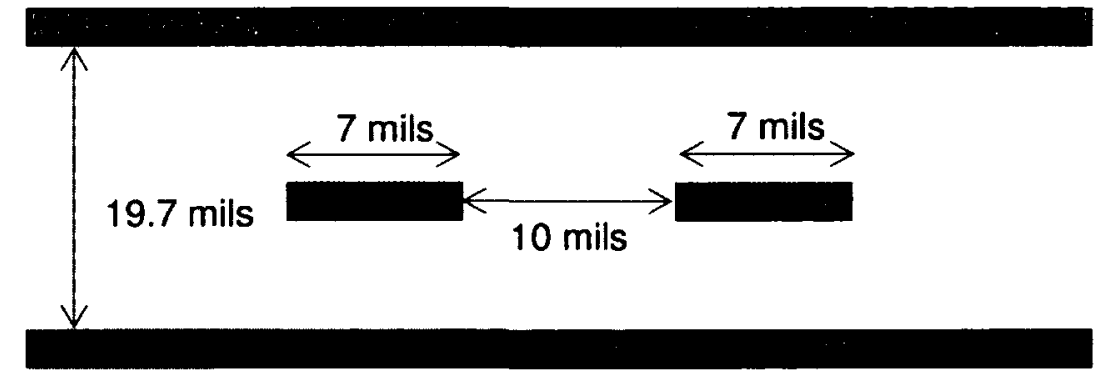

FIGURE 4.9: Differential trace geometry for example 3. 


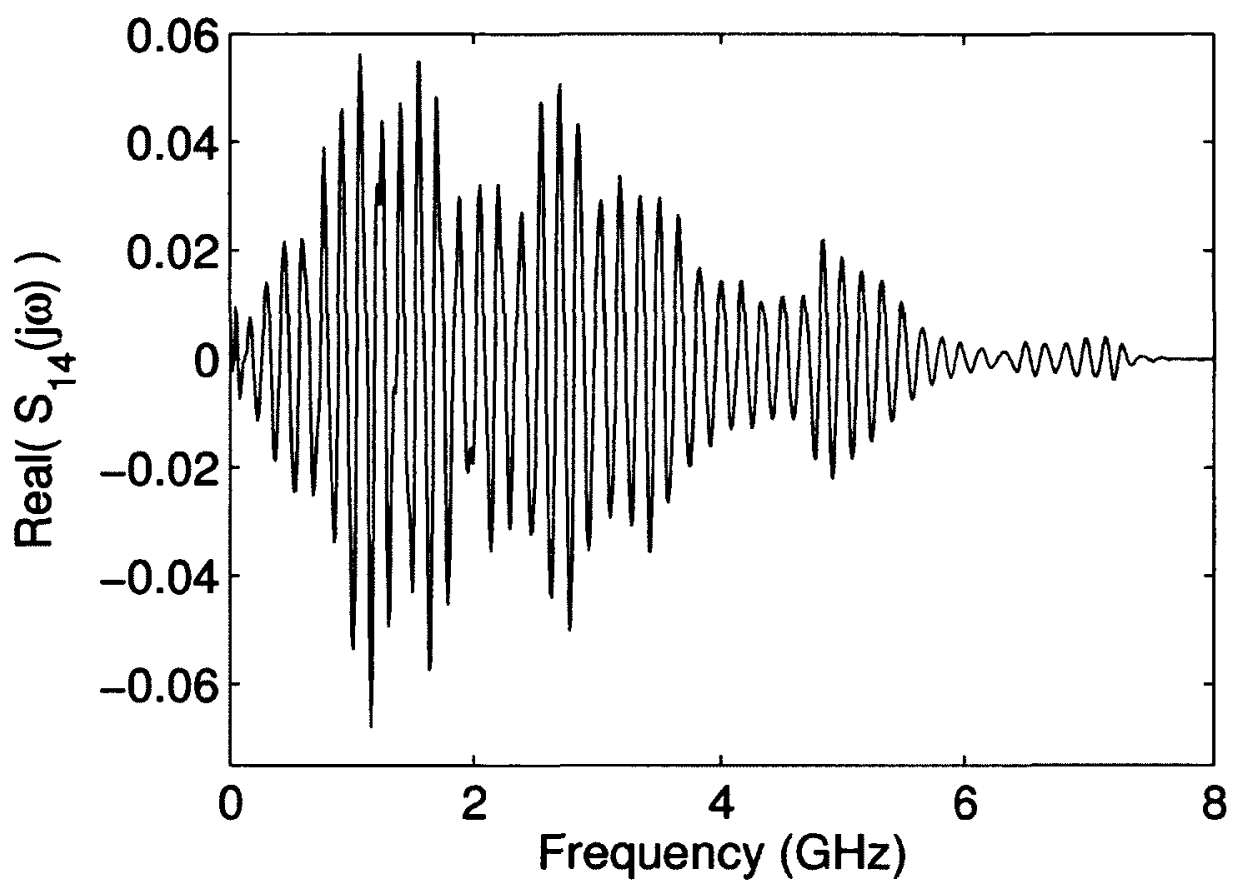

FIGURE 4.10: Scattering parameters for example 3.

Next, we discuss the passivity verification based on the proposed reduced search region algorithms of Section 4.3. Using the proposed search reduction algorithms based on the FDGEP and full-size Hamiltonian formulation, the maximum positive imaginary eigenvalue was found to be $26.64 \times 10^{9} \mathrm{rad} / \mathrm{s}$ (which corresponds to the maximum frequency for passivity violation search (i.e. $f_{\max }=26.64 \times 10^{9} /(2 \pi)=4.24$ $\mathrm{GHz})$ ). The corresponding eigenvalue spectrum (on the imaginary axis) is given in Fig. 4.11. Next, using Theorem 4.8, the model was found to be passive.

The above results are cross-verified by plotting a fine frequency-sweep of the magnitude spectrum of the scattering matrix and are given in Fig. 4.12(a) (shown in solid line). Also, an expanded view of the magnitude spectrum is shown in Fig. 4.12(b). As seen from Fig. 4.12(b), the macromodel is indeed passive, thus validating the proposed theory. 


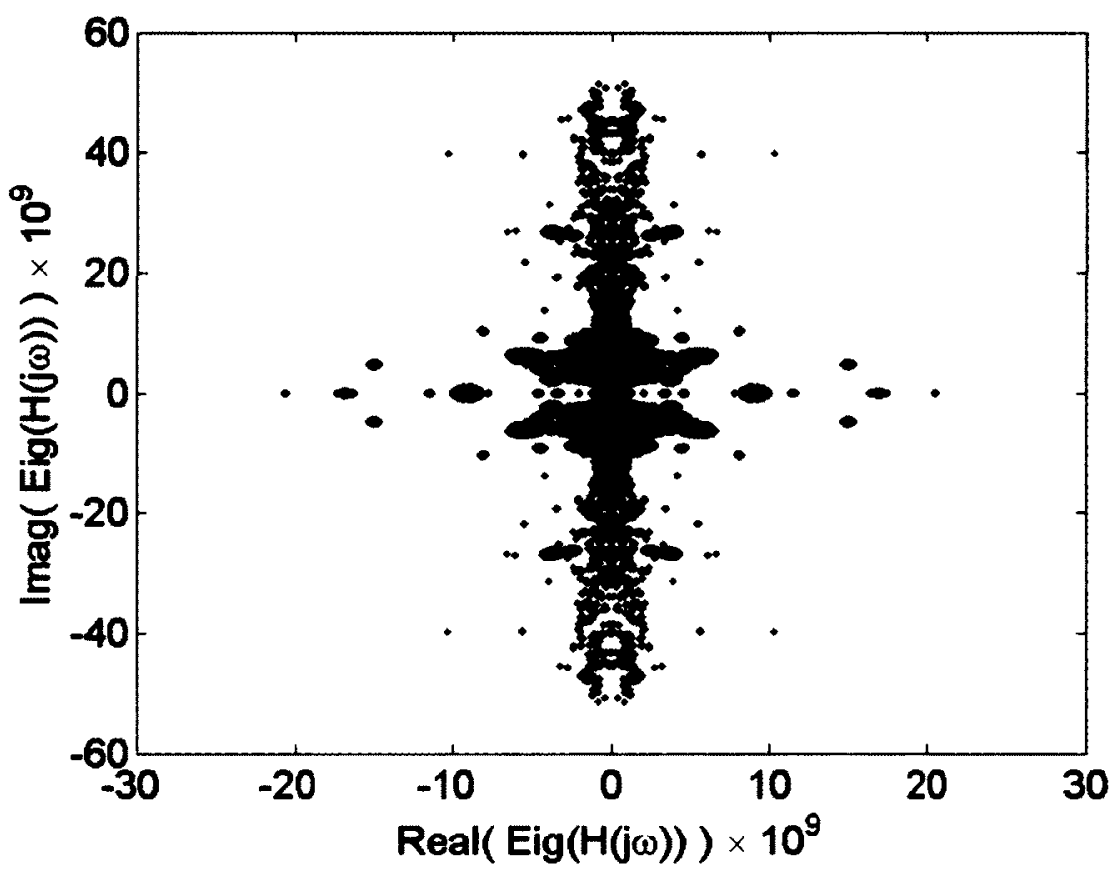

FIGURE 4.11: Eigenvalue spectrum of the full-size FDGEP for example 3.
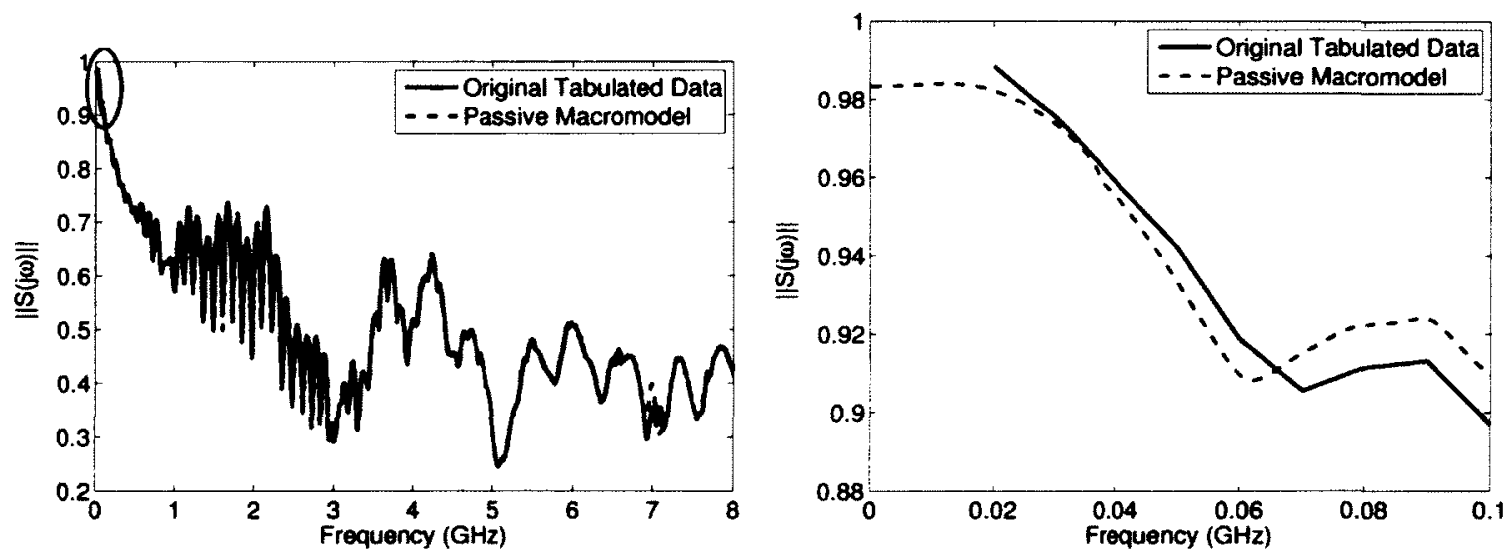

(a) Behavior of the norm of the scattering pa- (b) An expanded view of the circled region in Fig. rameters. 4.12(a).

FIGURE 4.12: Passivity verification of the DRFs for example 3. 
Next, the accuracy of the half-size FDGEP based approach is validated. The above experiments are repeated with the proposed half-size Hamiltonian formulation of Section 4.4, and identical results are obtained (i.e. the macromodel was found to be passive).

Table 4.2 gives a comparison of the CPU performance for obtaining an upper bound on the passivity verification region using the both the full-size and half-size FDGEP. It is clear from the table that a significant speed-up is obtained while performing passivity verification during each iteration of the passivity compensation process by using the proposed half-size FDGEP approach.

\subsection{Summary}

In this chapter, novel passivity verification and compensation algorithms for DRFbased macromodels obtained from tabulated scattering parameter data were introduced. For passivity verification, a FDGEP was developed that reduced the necessary passivity search region from the entire right-half plane to a single finite interval along the imaginary axis. In addition, a robust and fast half-size FDGEP was introduced that further reduced the passivity verification time over existing techniques. Detailed proofs of all Lemmas and Theorems were also presented. For the case of nonpassive macromodels, a passivity compensation scheme was introduced that iteratively perturbs the residues of the macromodel until it becomes passive. Several practical examples were also presented demonstrating the robustness of the proposed algorithms. 


\section{CHAPTER 5}

\section{Passive Macromodeling Algorithm for Admittance Parameter}

\section{Based Delayed Rational Functions}

In this chapter, passivity verification and compensation techniques are developed for DRF-based macromodels obtained in the admittance domain. The chapter is organized as follows. Section 5.1 provides motivation for the proposed work. Section 5.2 reviews DRF-based macromodeling techniques and passivity issues in the admittance domain. Next, section 5.3 presents necessary theorems and proofs for the proposed passivity verification algorithm. Section 5.4 provides the details of the passivity compensation algorithm. Section 5.5 presents numerical results that validate the proposed methods.

The work in this chapter first appeared in [67]. 


\subsection{Motivation}

In the previous chapter, passivity verification and compensation techniques were introduced for DRF-based macromodels of tabulated scattering parameter data. In the scattering domain, a well-matched line will have fewer reflective impulses in the timedomain impulse response. In turn, the tabulated data requires fewer DRF terms to obtain an accurate approximation and leads to a lower-order DRF macromodel. For this reason, it is preferable to perform DRF-based macromodeling in the scattering domain.

However, there are several scenarios where DRF macromodels are obtained in the admittance domain. Small errors from perturbations in the scattering domain can correspond to large errors in the admittance domain. Consequently, an accurate DRF approximation in the scattering domain may correspond to an inaccurate approximation in the admittance domain. Since transient analysis in SPICE simulators is typically performed in the admittance domain, this can lead to large errors in the simulated response. In addition, many EM solvers generate admittance domain data. Since it is more efficient and less prone to numerical errors from domain conversion, it is desirable to construct passive DRF-based macromodels directly in the domain of the given tabulated parameters.

Illustrative Example: In order to demonstrate the above issues, consider the network described by a single rational term in the scattering domain as

$$
S(s)=\frac{c}{s-p}
$$

where $c$ and $p$ are the residue and pole, respectively. Recall, that the admittance 
parameters are related to the scattering parameters (for the scalar case) as [48]

$$
Y(s)=Z_{0}^{-1 / 2}(I-S(s))(I+S(s))^{-1} Z_{0}^{-1 / 2}=\left(1 / Z_{0}\right)(I-S(s))(I+S(s))^{-1}
$$

where $Z_{0}$ is the characteristic impedance. Then, the admittance parameters for the example in (5.1) are

$$
Y(s)=\left(1 / Z_{0}\right)\left(\frac{s-p-c}{s-p+c}\right)=1 / Z_{0}-\frac{2 c / Z_{0}}{s-p+c}
$$

Next, note that

$$
\frac{\partial Y}{\partial c}=\frac{-1}{Z_{0}(s-p+c)}-\frac{(s-p-c)}{Z_{0}(s-p+c)^{2}}=\frac{-2}{Z_{0}\left(1+\frac{c}{s-p}\right)(s-p+c)}
$$

From (5.4) it becomes clear that large changes in the admittance parameters may occur as a result of small perturbations in the residue, $c$, corresponding to the scattering parameter based macromodel (i.e. for $c /(s-p) \approx-1$ ).

Next, consider the effect of perturbing the residue, $c$, on a numerical example. Let the original system have $c=-1.001, p=-1$, and $s=2 j \pi f$ where $f$ are the frequencies (scaled by $1 \times 10^{9}$ ). A plot of the norm of the scattering parameters is given in Figs. 5.1(a) and 5.1(b). It is clear from Fig. 5.1(b) that the original system is slightly nonpassive around $s=0$. Prominent passivity compensation techniques perturb the residue $c$ such that the model becomes passive. In this case, such a perturbation yields a passive model with $c=-0.999$ and $|d c|=0.002$. It is clear from Figs. 5.1(a) and 5.1(b) that the perturbed model is indeed passive. Next, plots of the real part of the scattering parameters for the original model and passive model are given in Figs. 5.2(a) and 5.2(b). It is clear that little error is introduced in the 


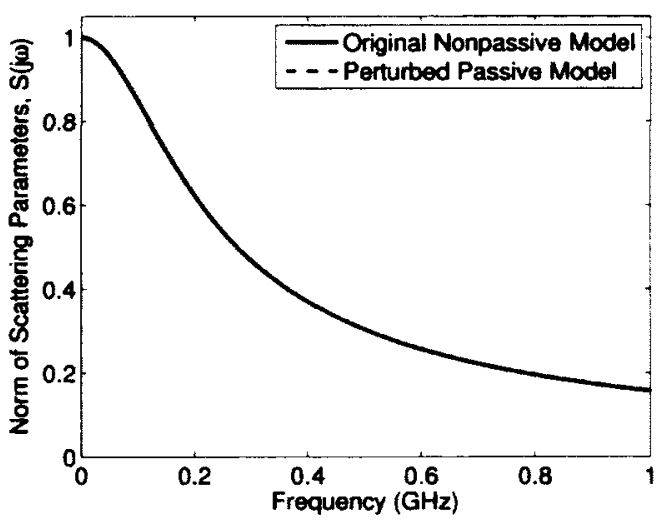

(a)

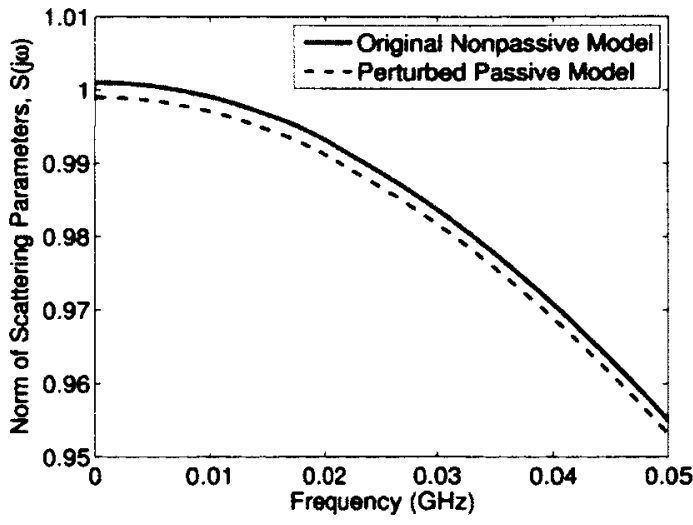

(b) An expanded view of Fig. 5.1(a).

FIGURE 5.1: Comparison of the norm of the scattering parameters in the frequency-domain.

scattering domain from the perturbation process.

Now consider the effect of the perturbation on the admittance parameters with $Z_{0}=50$. Figs. 5.3(a) and 5.3(b) show the admittance parameters for the original and passive models. It is clear from the expanded view in Fig. 5.3(b) that a large amount of error is introduced in the frequency-domain from the small perturbation to the scattering model.

To illustrate the effects of this small perturbation on the transient analysis, the original and passive models are connected in the circuit shown in Fig. 5.4. Transient analysis is performed using a step response with rise time of 0.5 ns. Fig. 5.5 shows the output voltage from the original and passive models. It is clear, due to the small perturbation of the residue in the scattering domain, a significant amount of error is introduced in the time-domain response.

To address the above issues, the techniques developed in the previous chapter for passivity verification and compensation of scattering parameter based DRF macromodels are modified for admittance-domain based DRF macromodels [67]. For this 


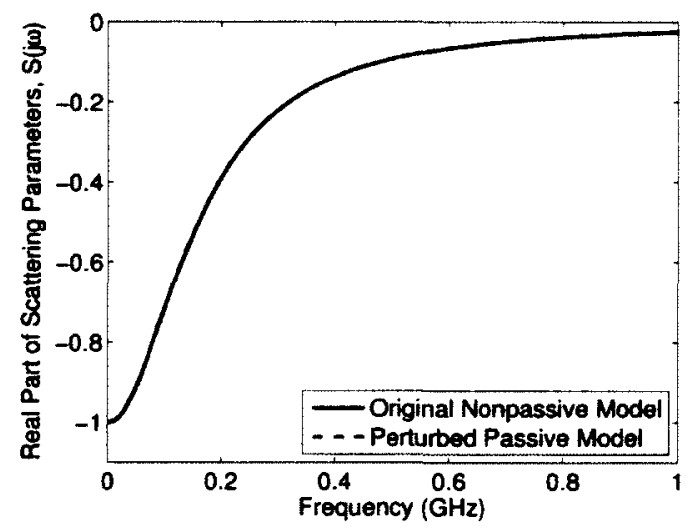

(a)

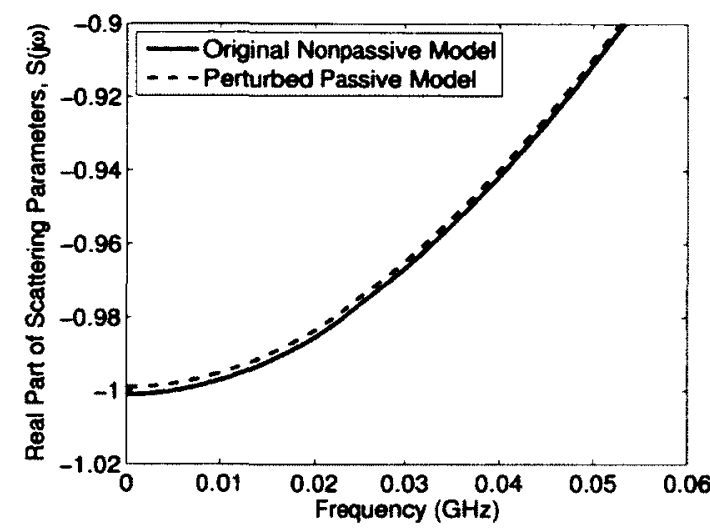

(b) An expanded view of Fig. 5.2(a).

FIGURE 5.2: Comparison of the real part of the scattering parameters in the frequency-domain.

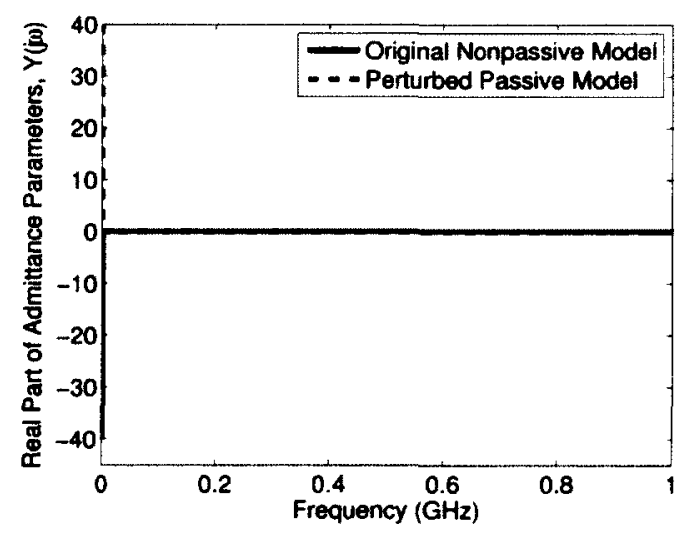

(a)

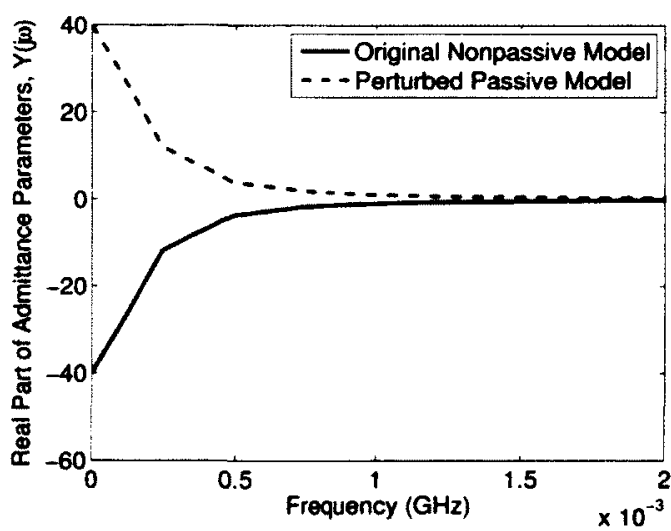

(b) An expanded view of Fig. 5.3(a).

FIGURE 5.3: Comparison of the real part of the admittance parameters in the frequency-domain. 


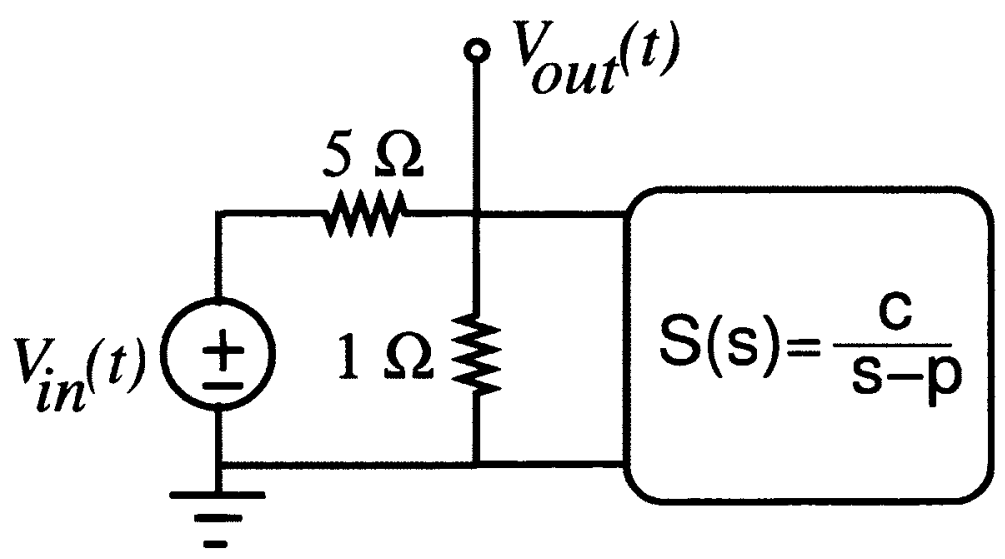

FIGURE 5.4: Circuit illustrating the effect of a small perturbation in the scattering domain model.

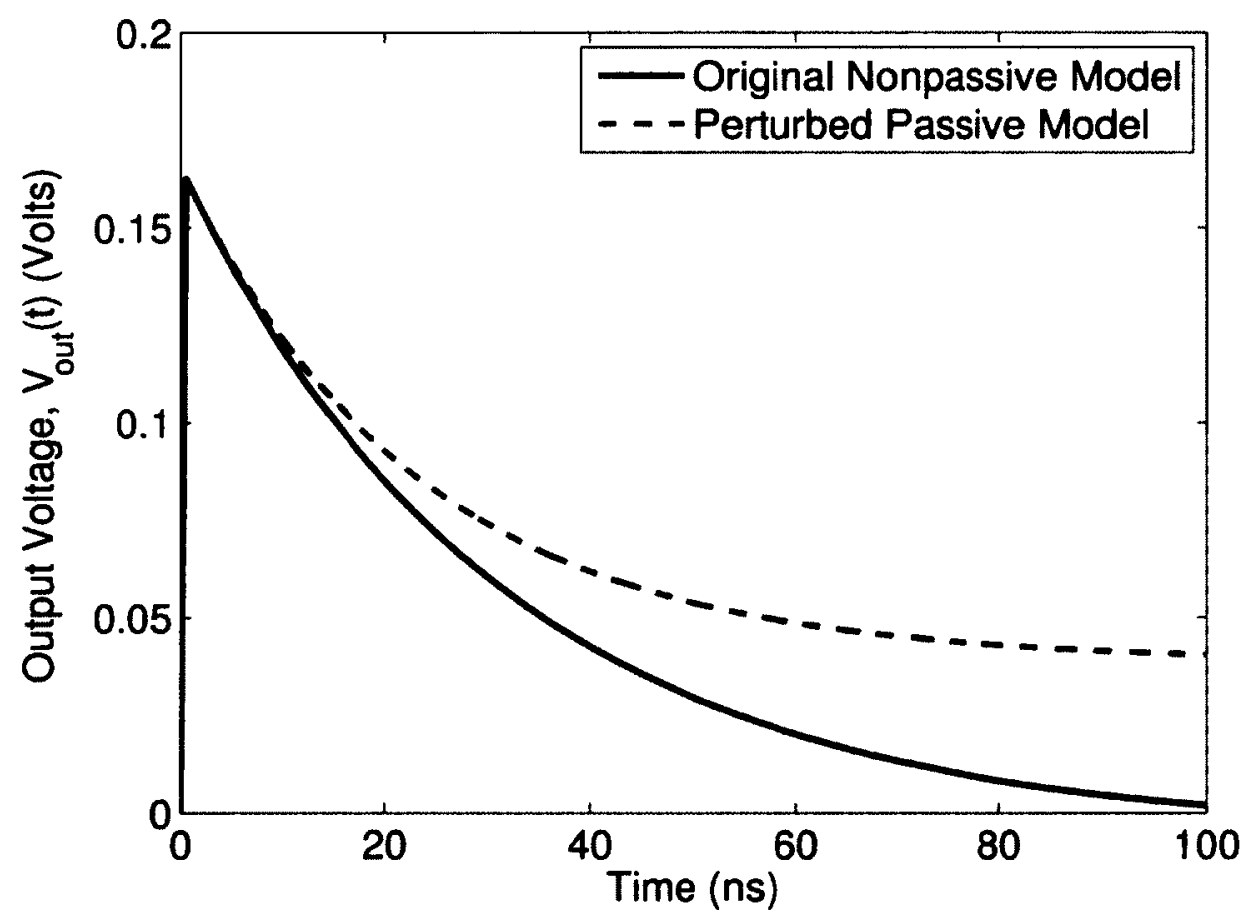

FIGURE 5.5: Time-domain response showing the effect of a small perturbation in scattering domain model. 
purpose, a new theorem is developed for fast identification of regions of passivity violation based on the solution of a quasi-periodic Frequency-Dependent Generalized Eigenvalue Problem (FDGEP), which reduces the necessary passivity verification search region from the entire right-half plane to just a single finite interval along the imaginary axis. In addition, a new passivity enforcement technique is presented that formulates the compensation process as a solution of linear matrix inequalities [87]. To minimize the error introduced in the perturbed macromodel, constraints are also imposed during the compensation process.

\subsection{Problem Formulation}

As discussed in Chapter 4, tabulated data has become a prominent technique for characterizing high-speed networks. In this chapter, networks characterized by admittance parameters are discussed.

To begin, consider an $h$-port network with port input voltages and currents $\left\{V_{i}\right\}$ and $\left\{I_{i}\right\}$, respectively (for $i=(1,2, \ldots, h)$ ). Then its admittance parameters are given by

$$
\left[\begin{array}{cccc}
Y_{11} & Y_{12} & \cdots & Y_{1 h} \\
\vdots & \vdots & \ddots & \vdots \\
Y_{h 1} & Y_{h 2} & \cdots & Y_{h h}
\end{array}\right]\left[\begin{array}{c}
V_{1} \\
\vdots \\
V_{h}
\end{array}\right]=\left[\begin{array}{c}
I_{1} \\
\vdots \\
I_{h}
\end{array}\right]
$$

where $Y_{i k}$ corresponds to the $(i, k)$ admittance element. For networks with long delays, macromodels are constructed by approximating each matrix entry, $Y_{i k}(s)$, using a DRF in the form [26]

$$
Y_{i k}(s)=\sum_{m=0}^{M} Q_{m}^{i, k}(s) e^{-s \tau_{m}}
$$


where $(i, k) \in\{1,2, \ldots, h\}$ refer to the corresponding matrix element, $M$ is the total number of delays in the network, and $\tau_{m}$ is the $m^{\text {th }}$ delay with $\tau_{0}=0$. Each of the $Q_{m}^{i, k}(s)$ are rational functions in the form

$$
Q_{m}^{i, k}(s)=R_{m, 0}^{i, k}+\sum_{n=1}^{N_{m}^{i, k}} \frac{R_{m, n}^{i, k}}{s-p_{m, n}^{i, k}}
$$

where for each $Q_{m}^{i, k}(s): N_{m}^{i, k}, R_{m, n}^{i, k}$, and $p_{m, n}^{i, k}$ are the total number of poles, the residues, and the poles, respectively. In time-domain state-space form, the DRFs become [27]

$$
\begin{aligned}
\dot{\boldsymbol{x}}(t) & =\boldsymbol{A} \boldsymbol{x}(t)+\boldsymbol{B} \boldsymbol{v}(t) \\
\boldsymbol{i}(t) & =\sum_{m=0}^{M} \boldsymbol{C}_{m} \boldsymbol{x}\left(t-\tau_{m}\right)+\sum_{m=0}^{M} \boldsymbol{D}_{m} \boldsymbol{v}\left(t-\tau_{m}\right)
\end{aligned}
$$

where $\boldsymbol{x}(t) \in \mathbb{R}^{P}$ represents the state variables, $\boldsymbol{A} \in \mathbb{R}^{P \times P}, \boldsymbol{B} \in \mathbb{R}^{P \times h}, \boldsymbol{C}_{m} \in \mathbb{R}^{h \times P}$, $\boldsymbol{D}_{m} \in \mathbb{R}^{h \times h}, P=(N \times M) \times h$, and $N=\sum_{i, k, m} N_{m}^{i, k}$. Then, the Laplace-domain transfer function for DRF-based macromodels is

$$
Y(s)=\sum_{m=0}^{M} C_{m}(s I-A)^{-1} B+\sum_{m=0}^{M} D_{m}
$$

To obtain DRFs from tabulated data, several techniques are available in the literature [26], [27], [30]. In each case, the delays are obtained by performing a timefrequency analysis on the data. Subsequently, a least squares fitting technique is applied to determine the remaining unknown poles and residues. To ensure the statespace Jordan canonical form has a direct meaning in the time-domain [5], the complex poles are forced to occur in conjugate pairs [26], [27], [30].

Stability in DRF-based macromodels is easily guaranteed during the fitting process. 
Poles are simply forced to the left-half of the complex plane prior to determining the residues. On the other hand, guaranteeing passivity is significantly more difficult. For a macromodel to be passive, it must satisfy the following 3 conditions in the frequency-domain [86].

a) $\boldsymbol{Y}(s)$ is analytic for all values of $s$ with $\mathfrak{R e}(s)>0$;

b) $\boldsymbol{Y}\left(s^{*}\right)=\boldsymbol{Y}^{*}(s)$, where the superscript ' $*$ ' is the complex conjugate operator;

c) $\left[\boldsymbol{Y}^{H}(s)+\boldsymbol{Y}(s)\right]$ is nonnegative definite for $\mathfrak{R e}(s)>0$. That is

$$
\boldsymbol{z}^{H}\left[\boldsymbol{Y}^{H}(s)+\boldsymbol{Y}(s)\right] \boldsymbol{z} \geq 0
$$

for all complex values of $s$ with $\mathfrak{R e}(s)>0$ and for any arbitrary vector $\boldsymbol{z}$. The superscript ' $H$ ' represents the Hermitian conjugate operator.

Condition a) and b) are always satisfied since the poles and residues are both real or come in complex conjugate pairs. However, direct verification of condition c) requires a robust frequency-sweep of the entire right-half of the complex plane. In many cases, this technique will miss passivity violations when the search grid is chosen too coarse. To address this issue for the case of rational functions without delays, passivity verification was formulated as an algebraic eigenvalue problem via a Hamiltonian matrix [97]. If the Hamiltonian matrix is not found to possess any pure imaginary eigenvalues, then the rational macromodel is passive. If the macromodel is found to be nonpassive, then passivity needs to be enforced to ensure accurate transient results. For rational functions without delays, passivity is enforced by perturbing the residues of the rational function [42]. However, for delayed rational functions, 
passivity verification and compensation becomes significantly more complex due to the exponential terms corresponding to delays.

Passivity verification and compensation of macromodels with time-delays was first addressed for the specific case of method of characteristics based transmission line macromodels with certain constraints [50]-[53]. For passivity verification, it was shown that the necessary search region could be reduced from the entire right-half plane to just the imaginary axis, provided the macromodel satisfied certain constraints. Next, passivity verification was formulated as a quasi-periodic FDGEP, which only needs to be solved over a single finite period [50], [51]. Consequently, the necessary search region for passivity verification was reduced from the entire right-half plane to only a finite interval along the imaginary axis. For nonpassive macromodels, passivity compensation techniques based on perturbation of the residues in the macromodel were developed [52], [53]. However, for admittance parameter based DRF macromodels, robust passivity verification and compensation remains a challenging task. To address this issue, the following sections develop passivity verification and compensation algorithms for DRF-based macromodels obtained in the admittance domain.

\subsection{Proposed Passivity Verification Algorithm}

Conventional passivity verification techniques perform a robust frequency-sweep of the eigenvalues of $\left[\boldsymbol{Y}(s)+\boldsymbol{Y}^{H}(s)\right]$ in the entire right-half plane. For practical purposes, this is computationally expensive and passivity violations may be missed if the search grid is chosen too coarse. To address this issue, the following passivity verification algorithm is proposed. 
First, the required search region is reduced from the entire right-half of the complex plane to only the imaginary axis. This is achieved by imposing a constraint on the DRF. Next, passivity verification is formulated as a frequency-dependent quasi-periodic eigenvalue problem. Consequently, the required search region is further reduced from the entire imaginary axis to only a single finite interval along the imaginary axis. Details are given in the following sections.

\subsubsection{Reduction of the Search Region to the Imaginary Axis}

To reduce the search region from the entire right-half of the complex plane to just the imaginary axis, the following lemma is proposed.

Lemma 5.1: If $Q_{m}^{i, k}(s)$ is asymptotically stable for all $(i, k) \in\{1,2, \ldots, h\}, m \in$ $(0,1, \ldots, M)$, then $\boldsymbol{Y}(s)$ defined by (5.6)-(5.7) satisfies the passivity conditions a) and $b$ ). In addition, if

$$
\lim _{s \rightarrow \infty} Q_{m}^{i, k}(s)=0, \quad \text { for } m \neq 0
$$

is satisfied, then condition c) need only be checked along the imaginary axis. That is, (5.10) can be replaced by the condition

$$
\boldsymbol{z}^{H}\left[\boldsymbol{Y}^{H}(j \omega)+\boldsymbol{Y}(j \omega)\right] \boldsymbol{z} \geq 0
$$

for $\omega \in \mathbb{R}$ and for any arbitrary vector $\boldsymbol{z}$.

Proof of Lemma 5.1: It is required to prove that under the assumptions of Lemma 5.1 , passivity conditions a), b), and c) are satisfied.

To begin, consider condition a). For the transfer function, $\boldsymbol{Y}(s)$, to be analytic for $\mathfrak{R e}(s)>0$ it is necessary that it satisfies the Cauchy-Riemann equations and has 
continuous partial derivatives in the right-half plane. Under the assumptions given in the Lemma, each of the $Q_{m}^{i, k}(s)$ is asymptotically stable and has no poles in the right-half plane. Consequently, each $Q_{m}^{i, k}(s)$ is analytic and satisfies the CauchyRiemann equations. In addition, from Proposition 4.2 the exponential functions $e^{-s \tau_{m}}$ are analytic for $\mathfrak{R e}(s)>0$ and satisfy the Cauchy-Riemann equations. Then, $\boldsymbol{Y}(s)$ defined by (5.6)-(5.7) is a sum of products of functions that each satisfy the Cauchy-Riemann equations and therefore, from Proposition 4.3 it must also satisfy the Cauchy-Riemann equations. As a result, $\boldsymbol{Y}(s)$ as defined in the proposed lemma is analytic for $\mathfrak{R e}(s)>0$.

Next, consider condition b). To see that this condition is satisfied, recall that during the fitting the complex poles are forced to occur as conjugate pairs [26], [27], [30]. Consequently, each of the rational functions in (5.7) can be written as a ratio of polynomials with real coefficients and it is clear that condition b) is satisfied.

The remainder of this section focuses on proving that condition c) reduces to the equivalent condition in (5.12). Specifically, it is desired to prove that if $\left[\boldsymbol{Y}^{H}(s)+\boldsymbol{Y}(s)\right]$ is positive real along the imaginary axis, then it is positive real for all $s$ with $\mathfrak{R e}(s)>0$.

To begin, let $\Omega$ be a semicircular area in the complex plane. Let the semicircular arc extend into the right-half plane with its bounding contour centered at the origin along the $j \omega$-axis. An illustration of this is shown in Fig. 4.1 for $s=\sigma+j \omega$. It is obvious that $\Omega$ will extend to cover the entire right-half plane as the radius of the contour $\mathcal{C}$ becomes arbitrarily large.

Next, using Proposition 4.3 note that since $\boldsymbol{Y}(s)$ satisfies the Cauchy-Riemann equations, $\boldsymbol{z}^{H}\left[\boldsymbol{Y}^{H}(s)+\boldsymbol{Y}(s)\right] \boldsymbol{z}$ also satisfies the Cauchy-Riemann equations and is analytic in $\Omega$ (since it is analytic for $\mathfrak{R e}(s)>0$ ). From the maximum $/$ minimum modulus theorem (Theorem 4.4), the minimum value for $\boldsymbol{z}^{H}\left[\boldsymbol{Y}^{H}(s)+\boldsymbol{Y}(s)\right] \boldsymbol{z}$ occurs 
on $s \in \mathcal{C}$. Therefore, if $\left[\boldsymbol{Y}^{H}(s)+\boldsymbol{Y}(s)\right]$ is positive definite for $s \in \mathcal{C}$, then it must be positive definite for $s \in \Omega$. Consequently, verification of positive definiteness in $\Omega$ reduces to verifying positive definiteness along the contour $\mathcal{C}$. Next, it is shown that as the radius of $\mathcal{C}$ approaches $\infty$, for DRFs that satisfy the assumptions in the Lemma it is only necessary to check the part of $\mathcal{C}$ that occurs along the $j \omega$-axis.

Note that if each $Q_{m}^{i, k}(s)$ is strict rational for $m>0$ (i.e. all the rational functions $Q_{m}^{i, k}(s)$ corresponding to nonzero delay $\tau_{m}$ ), then for each of the admittance matrix elements we get

$$
\left.Y_{i k}(s)\right|_{s \rightarrow \infty}=\left.Q_{0}^{i, k}(s)\right|_{s \rightarrow \infty}
$$

Therefore, the behavior of each $Y_{i k}(s)$ becomes asymptotically similar to the behavior of the stable rational function $Q_{0}^{i, k}(s)$ as the radius of the contour $\mathcal{C}$ approaches $\infty$. Consequently, the admittance matrix $\boldsymbol{Y}(s)$ becomes asymptotically independent of $\mathfrak{R e}(s)$ and behaves as a stable rational function. This leads to the result

$$
\left.\left[\boldsymbol{Y}^{H}(s)+\boldsymbol{Y}(s)\right]\right|_{s \rightarrow \infty}=\left.\left[\boldsymbol{Y}^{H}(s)+\boldsymbol{Y}(s)\right]\right|_{s \rightarrow j \infty}
$$

This means that, $\left[\boldsymbol{Y}^{H}(s)+\boldsymbol{Y}(s)\right]$ approaches a limiting value that is equal to the limit as $s \rightarrow j \infty$. From Theorem 4.4, since the minimum value of $\left[\boldsymbol{Y}^{H}(s)+\boldsymbol{Y}(s)\right]$ occurs on $\mathcal{C}$, this last statement implies the same minimum value must also occur on the $j \omega$-axis. Then, the condition $\boldsymbol{z}^{H}\left[\boldsymbol{Y}^{H}(s)+\boldsymbol{Y}(s)\right] \boldsymbol{z} \geq \mathbf{0}, \forall s \mid \mathfrak{R e}(s)=0$ implies that $\boldsymbol{z}^{H}\left[\boldsymbol{Y}^{H}(s)+\boldsymbol{Y}(s)\right] \boldsymbol{z} \geq 0, \forall s \mid \mathfrak{R e}(s)>0$ and this completes the proof.

Imposing the condition (5.11) of Lemma 5.1 requires that all direct coupling constants corresponding to delays vanish (i.e. $R_{m, 0}^{i, k}=0$ for $m>0$ ). Then, all the matrices corresponding to these coupling constants vanish as well (i.e. $\boldsymbol{D}_{m}=\mathbf{0}$ for $m>0$ ) 
and the Laplace-domain transfer function for the system given in (5.9) becomes

$$
\boldsymbol{Y}(s)=\sum_{m=0}^{M} \boldsymbol{C}_{m}(s \boldsymbol{I}-\boldsymbol{A})^{-1} \boldsymbol{B}+\boldsymbol{D}
$$

where $\boldsymbol{D}=\boldsymbol{D}_{0}$ corresponds to $\tau_{0}=0$. In addition, for passivity at $\omega=\infty$ it is also

necessary that $\left[\boldsymbol{D}+\boldsymbol{D}^{H}\right] \geq \mathbf{0}$. Both of these conditions can easily be enforced using any of the DRF approximation algorithms [68].

\subsubsection{Reduction to a Finite Search Region}

Although the above proposed lemma reduces the passivity verification region from the right-half of the complex plane to just the imaginary axis, this is still an infinite search region (from $-j \infty$ to $j \infty$ ). To further reduce the computational challenge, it is desired to reduce the search region from the entire imaginary axis to just a single finite interval along the imaginary axis. For this purpose, the following theorem is introduced, which formulates passivity verification as a quasi-periodic FDGEP.

Theorem 5.2: A delayed rational function based macromodel that satisfies the constraints imposed in Lemma 5.1 is passive if and only if there does not exist any purely imaginary eigenvalues for $s$ that satisfy the following frequency-dependent generalized eigenvalue problem

$$
s \boldsymbol{\xi}=\boldsymbol{H}(s) \boldsymbol{\xi}
$$

where

$$
\boldsymbol{H}(s)=\mathcal{M}+\sum_{m=0}^{M} \hat{\mathcal{M}}_{m} e^{-s \tau_{m}}+\sum_{m=0}^{M} \overline{\mathcal{M}}_{m} e^{s \tau_{m}}+\sum_{m, n=0}^{M} \mathcal{M}_{m n} e^{s\left(\tau_{m}-\tau_{n}\right)}
$$


and

$$
\begin{aligned}
& \mathcal{M}=\left[\begin{array}{cc}
\boldsymbol{A} & -\boldsymbol{B} \boldsymbol{R} \boldsymbol{B}^{H} \\
\mathbf{0} & -\boldsymbol{A}^{H}
\end{array}\right], \quad \hat{\mathcal{M}}_{m}=\left[\begin{array}{cc}
\boldsymbol{B R} \boldsymbol{C}_{m} & 0 \\
0 & 0
\end{array}\right] \\
& \overline{\mathcal{M}}_{m}=\left[\begin{array}{cc}
0 & 0 \\
0 & -C_{m}^{H} R B^{H}
\end{array}\right], \quad \mathcal{M}_{m n}=\left[\begin{array}{cc}
0 & 0 \\
C_{m}^{H} R C_{n} & 0
\end{array}\right] \text {, } \\
& \boldsymbol{R}=-\left(\boldsymbol{D}+\boldsymbol{D}^{H}\right)^{-1} \text {. }
\end{aligned}
$$

Proof of Theorem 5.2: Lemma 5.1 shows that for a DRF macromodel, passivity violations occur when a negative eigenvalue of $\left[\boldsymbol{Y}(s)+\boldsymbol{Y}^{H}(s)\right]$ is obtained on the $j \omega$-axis. Let $\left\{\lambda_{k}(j \omega)\right\}$ be the set of eigenvalues for $\left[\boldsymbol{Y}(s)+\boldsymbol{Y}^{H}(s)\right]$. Then it will be shown that the necessary and sufficient condition to have $\lambda_{k}\left(j \omega_{0}\right)=0$ for some $\omega_{0}$ is that $s=j \omega_{0}$ satisfies $(5.16)$.

To begin, assume that at some $s_{0}=j \omega_{0}, \lambda_{k}\left(j \omega_{0}\right)=0$. This implies that $\left[\boldsymbol{Y}\left(s_{0}\right)+\right.$ $\left.\boldsymbol{Y}^{H}\left(s_{0}\right)\right]$ is singular such that

$$
\left[\boldsymbol{Y}\left(s_{0}\right)+\boldsymbol{Y}^{H}\left(s_{0}\right)\right] \boldsymbol{\eta}=\mathbf{0}
$$

where $\boldsymbol{\eta}$ is the corresponding eigenvector. Substituting (5.15) into (5.19) and rearranging gives

$$
\boldsymbol{\eta}=-\boldsymbol{R}\left(\sum_{m=0}^{M} \boldsymbol{C}_{m} e^{-s \tau_{m}}\right) \boldsymbol{r}+\boldsymbol{R} \boldsymbol{B}^{T} \boldsymbol{\zeta}
$$


where $\boldsymbol{R}$ is defined in (5.18), $\boldsymbol{I}$ is the identity matrix, and $\boldsymbol{r}, \boldsymbol{\zeta}$ are defined as

$$
\begin{aligned}
& r=-\left(s_{0} \boldsymbol{I}-\boldsymbol{A}\right)^{-1} \boldsymbol{B} \boldsymbol{\eta} \\
& \boldsymbol{\zeta}=\left(-s_{0} \boldsymbol{I}-\boldsymbol{A}^{H}\right)^{-1}\left(\sum_{m=0}^{M} \boldsymbol{C}_{m}^{H} e^{s_{0} \tau_{m}}\right) \eta
\end{aligned}
$$

Rearranging (5.21a)-(5.21b) gives

$$
\begin{aligned}
& s_{0} \boldsymbol{r}=\boldsymbol{A} \boldsymbol{r}-\boldsymbol{B} \boldsymbol{\eta} \\
& s_{0} \boldsymbol{\zeta}=-\boldsymbol{A}^{H} \boldsymbol{\zeta}-\left(\sum_{m=0}^{M} \boldsymbol{C}_{m}^{H} e^{s_{0} \tau_{m}}\right) \boldsymbol{\eta} .
\end{aligned}
$$

Substituting (5.20) into (5.22) gives

$$
\begin{aligned}
& s_{0} \boldsymbol{r}=\boldsymbol{A} \boldsymbol{r}+\boldsymbol{B} \boldsymbol{R}\left(\sum_{m=0}^{M} \boldsymbol{C}_{m} e^{-s \tau_{m}}\right) \boldsymbol{r}-\boldsymbol{B R \boldsymbol { R }} \boldsymbol{B}^{H} \boldsymbol{\zeta} \\
& s_{0} \boldsymbol{\zeta}=-\boldsymbol{A}^{H} \boldsymbol{\zeta}+\left(\sum_{m=0}^{M} \boldsymbol{C}_{m}^{H} e^{s_{0} \tau_{m}}\right) \boldsymbol{R}\left(\sum_{n=0}^{M} \boldsymbol{C}_{n} e^{-s \tau_{n}}\right) \boldsymbol{r}-\left(\sum_{m=0}^{M} \boldsymbol{C}_{m}^{H} e^{\boldsymbol{s}_{0} \tau_{m}}\right) \boldsymbol{R} \boldsymbol{B}^{H} \boldsymbol{\zeta} .
\end{aligned}
$$

Defining

$$
\boldsymbol{\xi}=\left[\begin{array}{ll}
r^{H} & \boldsymbol{\zeta}^{H}
\end{array}\right]^{H}
$$

we obtain the desired result in (5.16) - (5.18). This proves the necessity condition.

In order to prove the sufficiency of the proposed theorem, assume that $s_{0}=j \omega_{0}$ satisfies

$$
s_{0} \boldsymbol{\xi}=\boldsymbol{H}\left(s_{0}\right) \boldsymbol{\xi}
$$

and then proceed in the reverse manner to show that $\left[\boldsymbol{Y}\left(s_{0}\right)+\boldsymbol{Y}^{H}\left(s_{0}\right)\right]$ is singular. 
From the above analysis, it is obvious that if there are no imaginary eigenvalues satisfying (5.16), then there are no imaginary eigenvalues that satisfy $\left[\boldsymbol{Y}(s)+\boldsymbol{Y}^{H}(s)\right]=\mathbf{0}$ (i.e. $\left.\left\{\lambda_{k}(j \omega) \neq 0 \quad \forall \omega \in \mathbb{R}\right\}\right)$. This implies that if the eigenvalues, $\left\{\lambda_{k}(j \omega)\right\}$, are positive for some values of $\omega$, then they are positive for all values of $\omega$. Therefore, $\left[\boldsymbol{Y}(s)+\boldsymbol{Y}^{H}(s)\right]$ has only positive eigenvalues, $\boldsymbol{Y}(s)$ is positive real, and consequently, $\boldsymbol{Y}(s)$ is passive. This completes the proof.

As previously mentioned, the FDGEP described by (5.16) is quasi-periodic. This property arises because $\boldsymbol{H}(s)$ is composed of a summation of constant matrices that are independent of $s$, while the summation coefficients are scalar functions of $s$ described by the exponentials $e^{ \pm s \tau_{m}}$ and $e^{s\left(\tau_{m}-\tau_{n}\right)}$, for $m, n=(0,1, \ldots, M)$. The restriction that $s=j \omega$ means the exponentials are all periodic in $\omega$ with different periods and consequently, $\boldsymbol{H}(s)$ is a quasi-periodic function of $\omega$. It follows that the eigenvalues of $\boldsymbol{H}(s)$ are also all quasi-periodic along the imaginary axis [51]. It is clear then that, we need only search along a single period of the frequency-dependent eigenvalue problem to detect all passivity violations.

The above theorem provides a theoretical framework that reduces the search region from the imaginary axis to a single, finite interval along the imaginary axis. However, it does not provide details on locating regions of passivity violation. The following section addresses this issue.

\subsubsection{Identification of Passivity Violation Regions}

In the previous section, Theorem 5.2 presented the necessary and sufficient conditions for a DRF macromodel to become nonpassive. Specifically, it was shown that the eigenvalues of the FDGEP in (5.16) become pure imaginary values when the 
eigenvalues of $\left[\boldsymbol{Y}(s)+\boldsymbol{Y}^{H}(s)\right]$ change sign. However, for passivity compensation it also necessary to identify and quantify the regions of passivity violations. For this purpose, the following theorem is introduced.

Theorem 5.3: The eigenvalue(s) of $\left[\boldsymbol{Y}^{H}\left(s_{0}\right)+\boldsymbol{Y}\left(s_{0}\right)\right]$ for a nonpassive delayed rational function based macromodel vanish at the point(s) $s_{0}=j \omega_{0}$ that satisfy (5.16) along the imaginary axis.

Proof of Theorem 5.3: From Theorem 5.2, if $s_{0}$ is an imaginary value satisfying (5.16), then the matrix $\left[\boldsymbol{Y}^{H}\left(s_{0}\right)+\boldsymbol{Y}\left(s_{0}\right)\right]$ is singular and the system becomes nonpassive.

From Theorem 5.2 and Theorem 5.3 it is clear that the regions of passivity violation for DRF macromodels are delimited by the pure imaginary eigenvalues that satisfy the FDGEP in (5.16). To solve the frequency-dependent generalized eigenvalue in (5.16), several techniques are available in the literature [50]-[52].

\subsection{Proposed Passivity Compensation Algorithm}

If the macromodel is found to be nonpassive, then the next step is to enforce passivity. In this section, the theoretical framework and necessary implementation details are presented to compensate for passivity violations in DRF macromodels.

\subsubsection{Perturbation of Residues}

To compensate for the passivity violations in the DRF macromodel, the residues are perturbed such that negative eigenvalues of $\left[\boldsymbol{Y}^{H}(s)+\boldsymbol{Y}(s)\right]$ become positive. The 
desired residue perturbation is obtained via the solution of linear matrix inequalities. Details are as follows.

To simplify the following analysis, assume there is a single passivity violation region in the DRF macromodel. Let $\omega_{l}$ and $\omega_{u}$ be the lower and upper bounds of this region, respectively. Furthermore, assume that the maximum violation (corresponding to the most negative eigenvalue of $\left[\boldsymbol{Y}^{H}(j \omega)+\boldsymbol{Y}(j \omega)\right]$ on $\left.\left(j \omega_{l}, j \omega_{u}\right)\right)$ has been found and occurs at frequency $\omega=\omega_{\max }$. The goal of passivity compensation is to perturb the residue matrices, $\boldsymbol{C}_{\boldsymbol{m}}$, such that $\left[\boldsymbol{Y}^{\boldsymbol{H}}(j \omega)+\boldsymbol{Y}(j \omega)\right]$ becomes positive real on $\left(j \omega_{l}, j \omega_{u}\right)$ and the model becomes passive. Let $\hat{\boldsymbol{C}}_{m}$ correspond to the $m^{\text {th }}$ residue matrix in the perturbed model such that

$$
\hat{C}_{m}=C_{m}+\Delta C_{m}
$$

where $\Delta C_{m}$ is the desired perturbation of the $m^{\text {th }}$ matrix. Then, the transfer function for the perturbed model is given by

$$
\hat{\boldsymbol{Y}}(j \omega)=\boldsymbol{Y}(j \omega)+\Delta \boldsymbol{Y}(j \omega)
$$

where

$$
\Delta \boldsymbol{Y}(j \omega)=\sum_{m=0}^{M} e^{-j \omega \tau_{m}} \Delta \boldsymbol{C}_{m}(j \omega \boldsymbol{I}-\boldsymbol{A})^{-1} \boldsymbol{B}
$$

and $\boldsymbol{Y}(j \omega)$ is defined in (5.15). It is desired to perturb the model at the frequency of maximum passivity violation, $\omega_{\max }$. This leads to the linear matrix inequality

$$
\hat{\boldsymbol{Y}}^{H}\left(j \omega_{\max }\right)+\hat{\boldsymbol{Y}}\left(j \omega_{\max }\right) \geq \mathbf{0}
$$




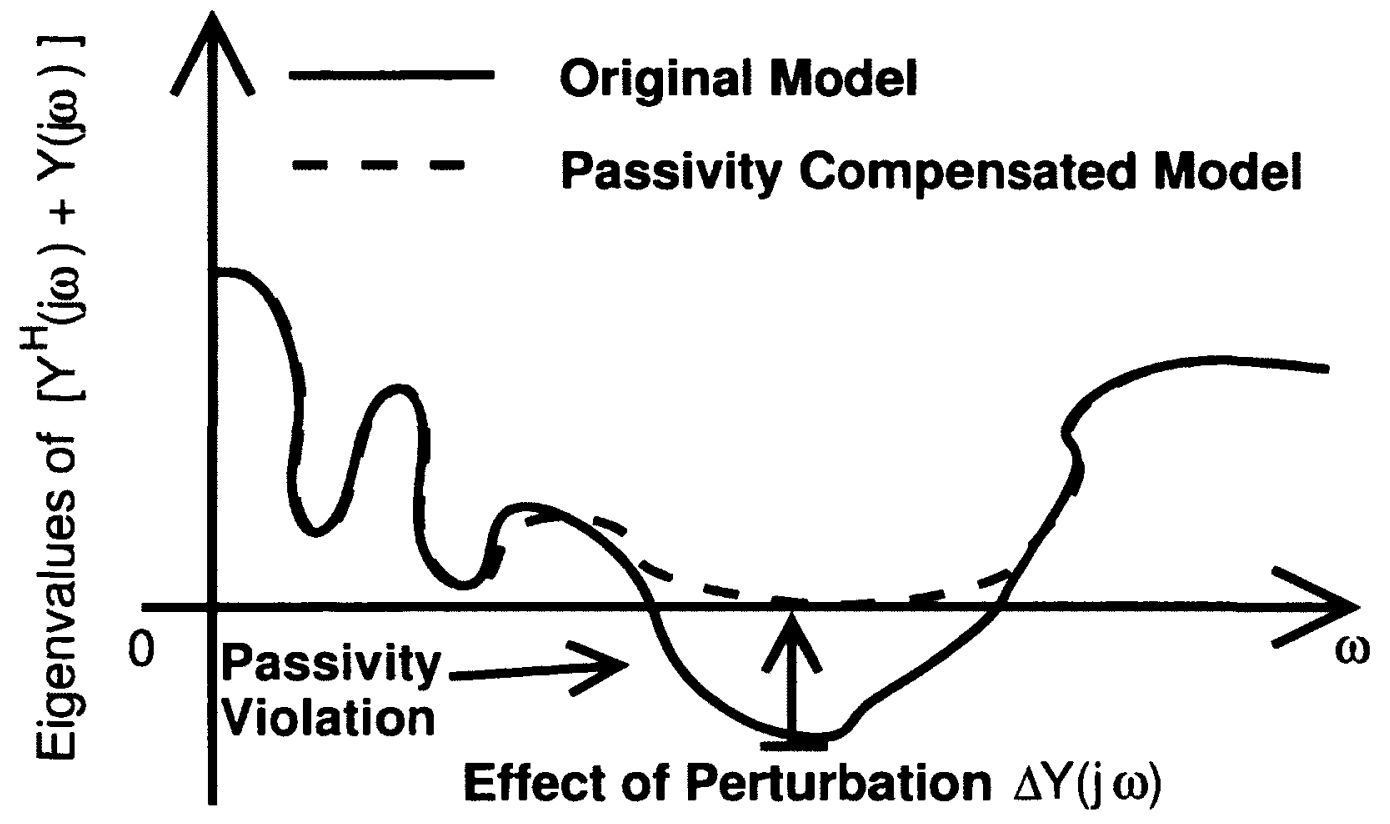

FIGURE 5.6: Illustration of the passivity compensation process.

Equivalently,

$$
\boldsymbol{Y}^{H}\left(j \omega_{\max }\right)+\boldsymbol{Y}\left(j \omega_{\max }\right)+\sum_{m=0}^{M} \Delta \boldsymbol{C}_{m} \boldsymbol{F}_{m}\left(j \omega_{\max }\right)+\sum_{m=0}^{M} \boldsymbol{F}_{m}^{H}\left(j \omega_{\max }\right) \Delta \boldsymbol{C}_{m}^{T} \geq \mathbf{0}
$$

where $\boldsymbol{F}_{m}\left(j \omega_{\max }\right)=e^{-j \omega_{\max } \tau_{m}}\left(j \omega_{\max } \boldsymbol{I}-\boldsymbol{A}\right)^{-1} \boldsymbol{B}$ and the Hermitian conjugate operator on $\Delta C_{m}$ reduces to the transpose operator, ' $T$ ', since the $\Delta C_{m}$ are all real.

The formulation in (5.30) is linear in each of the $\Delta C_{m}$ and consequently, can easily be solved using standard linear matrix inequality techniques [87]. The resulting model described by (5.27) now satisfies the necessary and sufficient conditions to be passive. An illustration of the passivity compensation is shown in Fig. 5.6.

In many cases, $\left[\boldsymbol{Y}^{H}(s)+\boldsymbol{Y}(s)\right]$ is not necessarily a purely real matrix (this is typically the case for unsymmetrical macromodels). In practice, this can be a problem since many linear matrix inequality solvers cannot always directly handle complex 
functions. To address this issue, we make use of Lemma 2.7, re-stated here for convenience.

Lemma 5.4: A complex Hermitian matrix, $\boldsymbol{\Upsilon}$, is positive semi-definite if and only if

$$
\left[\begin{array}{cc}
\mathfrak{R e}(\boldsymbol{\Upsilon}) & \mathfrak{I m}(\mathbf{\Upsilon}) \\
-\mathfrak{I m}(\boldsymbol{\Upsilon}) & \mathfrak{R e}(\mathbf{\Upsilon})
\end{array}\right] \geq \mathbf{0}
$$

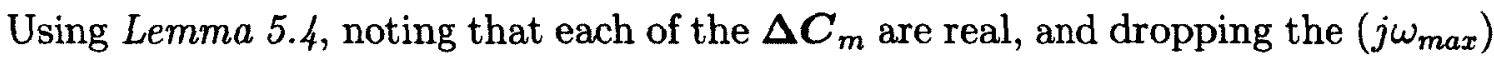
terms, the linear matrix inequality in (5.30) becomes equivalent to

$$
\begin{array}{r}
\sum_{m=0}^{M}\left\{\left[\begin{array}{cc}
\Delta \boldsymbol{C}_{m} & \mathbf{0} \\
\mathbf{0} & \Delta \boldsymbol{C}_{m}
\end{array}\right]\left[\begin{array}{cc}
\boldsymbol{F}_{R, m} & \boldsymbol{F}_{I, m} \\
-\boldsymbol{F}_{I, m} & \boldsymbol{F}_{R, m}
\end{array}\right]+\left[\begin{array}{cc}
\boldsymbol{F}_{R, m} & \boldsymbol{F}_{I, m} \\
-\boldsymbol{F}_{I, m} & \boldsymbol{F}_{R, m}
\end{array}\right]^{T}\left[\begin{array}{cc}
\Delta \boldsymbol{C}_{m}^{T} & \mathbf{0} \\
\mathbf{0} & \Delta \boldsymbol{C}_{m}^{T}
\end{array}\right]\right\} \\
+\left[\begin{array}{cc}
\boldsymbol{Y}_{R} & \boldsymbol{Y}_{I} \\
-\boldsymbol{Y}_{I} & \boldsymbol{Y}_{R}
\end{array}\right]^{T}+\left[\begin{array}{cc}
\boldsymbol{Y}_{R} & \boldsymbol{Y}_{I} \\
-\boldsymbol{Y}_{I} & \boldsymbol{Y}_{R}
\end{array}\right] \geq \mathbf{0}
\end{array}
$$

where $\boldsymbol{F}_{m}=\boldsymbol{F}_{R, m}+j \boldsymbol{F}_{I, m}$ and $\boldsymbol{Y}=\boldsymbol{Y}_{R}+j \boldsymbol{Y}_{I}$.

The above analysis assumed that only a single perturbation is necessary to remove all the passivity violations in the region $\left(j \omega_{l}, j \omega_{u}\right)$. However, in practice this process may cause new passivity violation regions to occur in the model. Consequently, passivity enforcement may require several iterations, where at each iteration the regions of passivity violation are calculated using the technique described in Section 5.3.

The proposed passivity compensation algorithm can easily be modified to compensate DRF macromodels with multiple regions of passivity violation. The linear matrix inequality in (5.32) is formulated at the frequency point of maximum violation for each region with a passivity violation. Subsequently, all the linear matrix inequalities 
are solved at once.

\subsubsection{Error Control}

In the previous section, a passivity compensation algorithm was developed that perturbed the residues in the DRF macromodel. However, it did not provide restrictions on the error introduced in the response. For this purpose, the perturbation matrices $\left\{\Delta C_{m}\right\}$ are related to the $L_{2}$-norm of error introduced in the response $\Delta \boldsymbol{Y}(j \omega)$. To see this note that the $L_{2}$-norm of $\boldsymbol{\Delta Y}$ (dropping the $(j \omega)$ ) can be written as [53]

$$
\|\Delta \boldsymbol{Y}\|_{2}^{2}=\left\|\sum_{m=0}^{M} \Delta \boldsymbol{Y}_{m}\right\|_{2}^{2} \leq \sum_{m=0}^{M}\left\|\Delta \boldsymbol{Y}_{m}\right\|_{2}^{2}=\sum_{m=0}^{M} \int_{-\infty}^{\infty}\left\|\Delta \boldsymbol{Y}_{m}\right\|_{F}^{2} d \omega
$$

where $\|\cdot\|_{2}$ and $\|\cdot\|_{F}$ are the $L_{2}$-norm and the Frobenius norm of the argument, respectively. Substituting in $\Delta \boldsymbol{Y}_{m}=e^{-j \omega \tau_{m}} \Delta \boldsymbol{C}_{m}(j \omega \boldsymbol{I}-\boldsymbol{A})^{-1} \boldsymbol{B}$ gives

$$
\|\Delta \boldsymbol{Y}\|_{2}^{2} \leq \sum_{m=0}^{M} \int_{-\infty}^{\infty}\left\|e^{-j \omega \tau_{m}} \Delta \boldsymbol{C}_{m}(j \omega \boldsymbol{I}-\boldsymbol{A})^{-1} \boldsymbol{B}\right\|_{F}^{2} d \omega=\sum_{m=0}^{M} \operatorname{trace}\left(\Delta \boldsymbol{C}_{m} \boldsymbol{P} \Delta \boldsymbol{C}_{m}^{T}\right)
$$

Here, $\boldsymbol{P}$ is the controllability Grammian obtained from solving the Lyapunov equation given by [91]

$$
\boldsymbol{A P}+\boldsymbol{P} \boldsymbol{A}^{T}+\boldsymbol{B} B^{T}=\mathbf{0}
$$

It is clear then that, minimizing the sum of traces of $\left(\Delta C_{m} P \Delta C_{m}^{T}\right)$ will in turn minimize the error introduced in the time-domain and frequency-domain responses of the perturbed DRF macromodels. Combining this result with the passivity compensation algorithm developed in the previous section, passivity enforcement with error control 
reduces to solving for the $\Delta C_{m}$ in the following problem

$$
\min _{\left\{\Delta C_{m}\right\}} \sum_{m=0}^{M} \operatorname{trace}\left(\Delta C_{m} P \Delta C_{m}^{T}\right), \quad \text { subject to (5.32) }
$$

Pseudocode summarizing the passive macromodeling scheme for DRF macromodels from tabulated admittance data is given in Algorithm 2.

Algorithm 2 Pseudocode for passive macromodeling scheme

Step 1: Formulate the matrices of the quasi-periodic frequency-dependent generalized eigenvalue in (5.16).

Step 2: Check if the macromodel is passive using the technique presented in Section 5.3.3. If it is nonpassive go to Step 3, otherwise, the DRF macromodel is passive and we are done.

Step 3: Identify the regions of passivity violation using the techniques discussed in Section 5.3.3.

Step 4: Apply passivity enforcement using the constrained linear matrix inequality formulated in (5.36) to solve for the perturbations in the residue matrices, $\left\{\Delta C_{m}\right\}$.

Step 5: Check if the macromodel is passive using the technique presented in Section 5.3.3. If it is nonpassive go back to Step 3, otherwise, the DRF macromodel is passive and we are done.

\subsection{Numerical Results}

In this section, the proposed passivity verification and compensation algorithms are validated on 3 industrial examples. All testing is performed on an Intel $i 7-9202.67$ $\mathrm{GHz}$ processor with $12 \mathrm{~Gb}$ of RAM. The constrained linear matrix inequalities are solved using SeDuMi [98] with Yalmip [99]. 


\subsubsection{Example 1}

In order to validate the proposed algorithm, it was tested on a 2-port frequencydependent transmission line network characterized by tabulated admittance data. The tabulated data was fit accurately up to $1.5 \mathrm{GHz}$ using DRFs [30]. Fitting each matrix element separately, it required 44 poles and 8 delay terms to accurately match the frequency-domain response data.

Next, the proposed passivity verification algorithm was validated. Using the FDGEP in (5.16) and efficient FDGEP solution techniques [50]-[52] the maximum pure imaginary eigenvalue was found to be $5.8874 \times 10^{9} \mathrm{rad} / \mathrm{s}$ [which corresponds to the maximum frequency of the passivity verification search region (i.e. $f_{\max }=$ $5.8874 / 2 \pi=0.9370 \mathrm{GHz})$ ]. Fig. 5.7 shows the corresponding eigenvalue spectrum for the FDGEP. Then, using Theorem 5.3 the model was found to be nonpassive on the intervals $[0.5819,2.4927] \times 10^{-3}$ and $[3.1844,5.9676] \times 10^{-3} \mathrm{GHz}$.

To cross-verify the above results, a robust frequency-sweep of the eigenvalues of $\left[\boldsymbol{Y}^{H}(j \omega)+\boldsymbol{Y}(j \omega)\right]$ was performed along the imaginary axis. Fig. 5.8 shows a plot of the resulting eigenvalues of $\left[\boldsymbol{Y}^{H}(j \omega)+\boldsymbol{Y}(j \omega)\right]$. It is clear from Fig. 5.8(b) that the eigenvalues of $\left[\boldsymbol{Y}^{H}(j \omega)+\boldsymbol{Y}(j \omega)\right]$ become negative (corresponding to passivity violations) over the above intervals, in agreement with the passivity violations obtained from the proposed algorithm.

Since the macromodel was found to be nonpassive, the proposed passivity compensation algorithm is applied. From Fig. 5.8, it can be seen that the proposed compensation process was successful and the compensated macromodel is passive. This was cross-verified using the proposed passivity verification algorithm, which found no passivity violations in the compensated macromodel. In addition, comparisons 


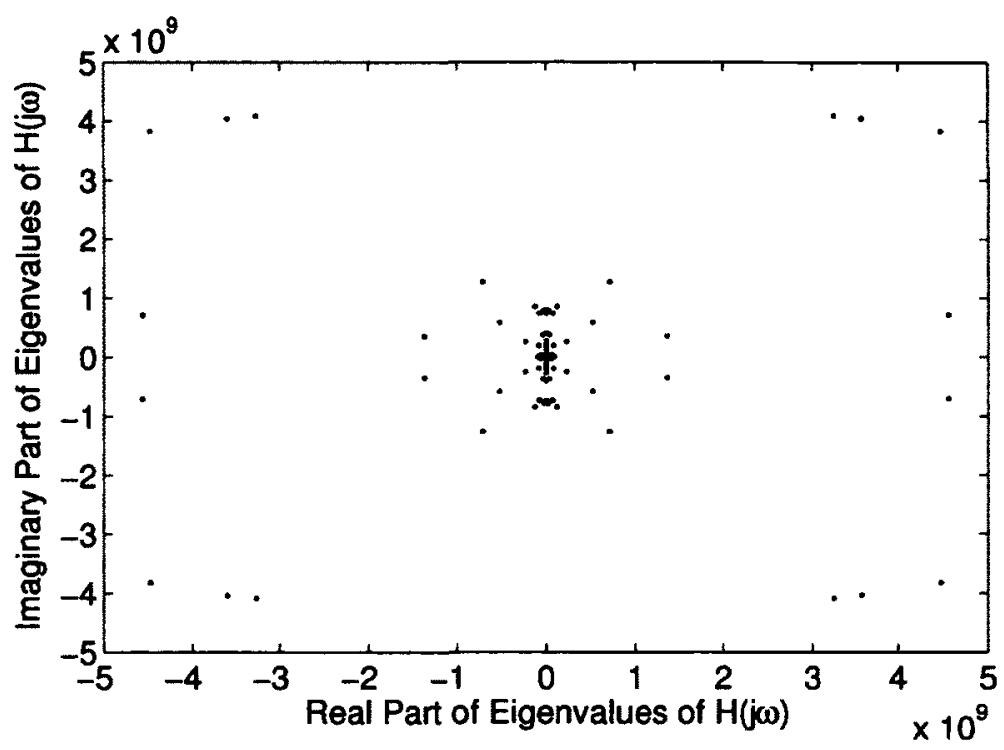

FIGURE 5.7: Eigenvalue spectrum of the FDGEP for example 1.

of the admittance parameters between the original and compensated macromodels are given in Fig. 5.9. It is clear from the figure that the error introduced in the frequency-domain responses of the compensated macromodel is negligible.

Next, the accuracy of the proposed algorithm is validated in the time-domain. The macromodel was connected to passive terminations as shown in Fig. 5.10. It was excited with a $20 \mathrm{~ns}$ wide pulse with $0.1 \mathrm{~ns}$ rise/fall times. It required 7.40 and 7.41 seconds to simulate the original and passive macromodels, respectively. A plot of the voltage at the output port is given in Fig. 5.11. As seen, the original and compensated DRF macromodels are in good agreement.

\subsubsection{Example 2}

In this example, a four-port interconnect network characterized by tabulated admittance data provided up to $10 \mathrm{GHz}$ is considered. The data was approximated using a DRF [30]. The macromodel required a total of 144 poles and 11 delay terms (each 


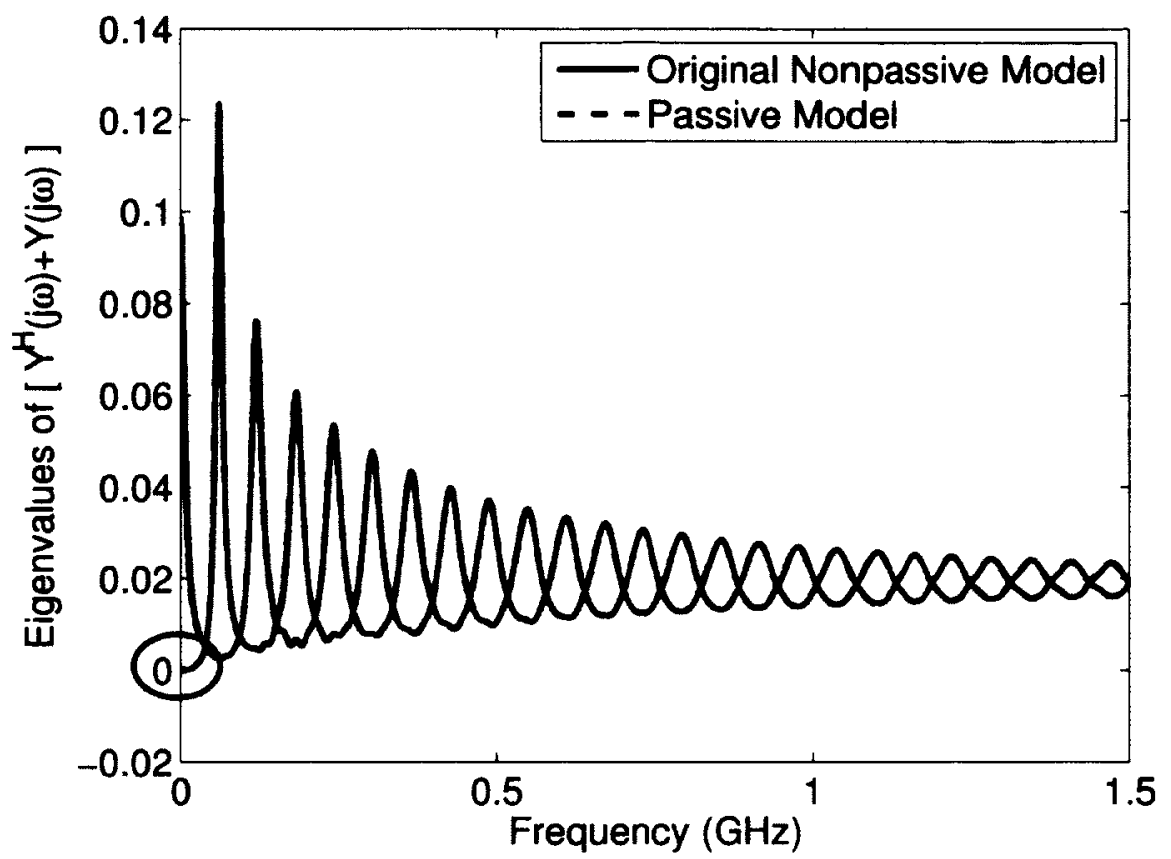

(a) Eigenvalue spectrum of the macromodels between 0 and $1.5 \mathrm{GHz}$ (the graphs of the Original Nonpassive Model and Passive Model are overlapping).

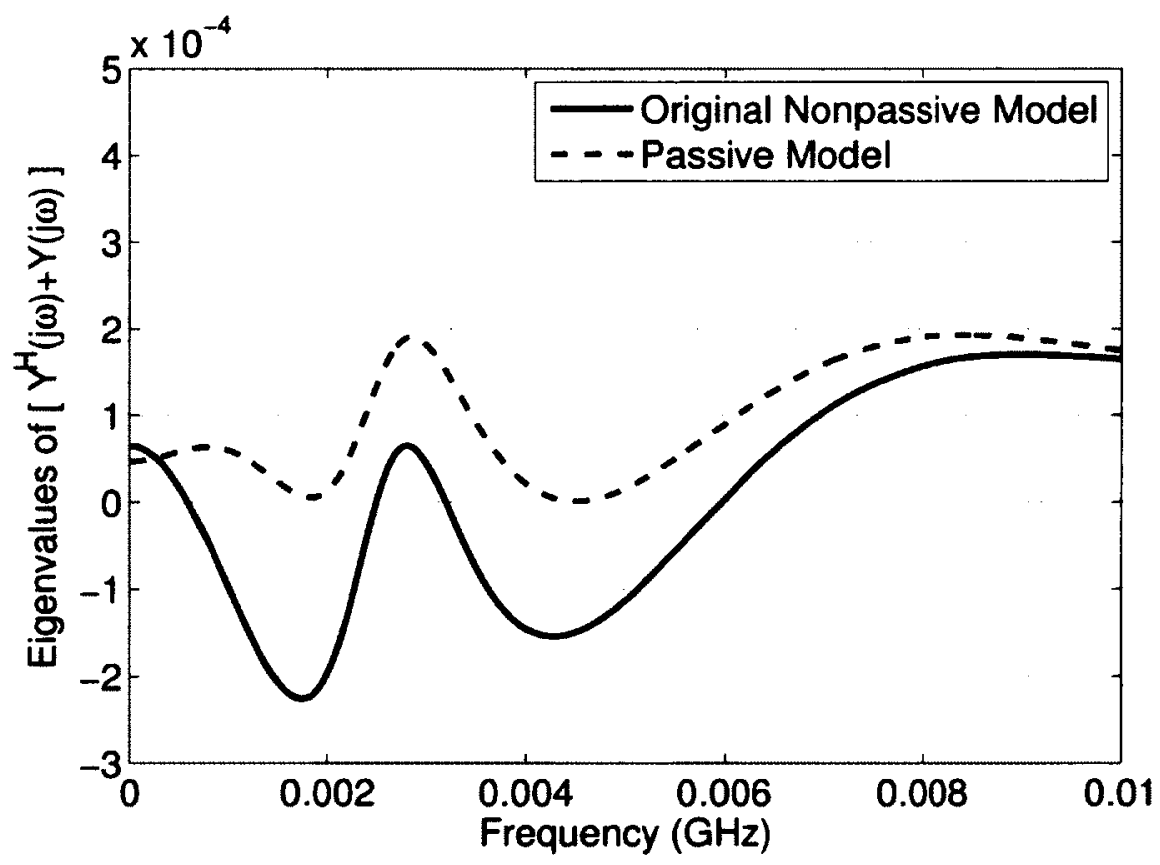

(b) Expanded view between 0 and $0.01 \mathrm{GHz}$.

FIGURE 5.8: Real eigenvalues of $\left[\boldsymbol{Y}^{H}(j \omega)+\boldsymbol{Y}(j \omega)\right]$ for example 1 . 


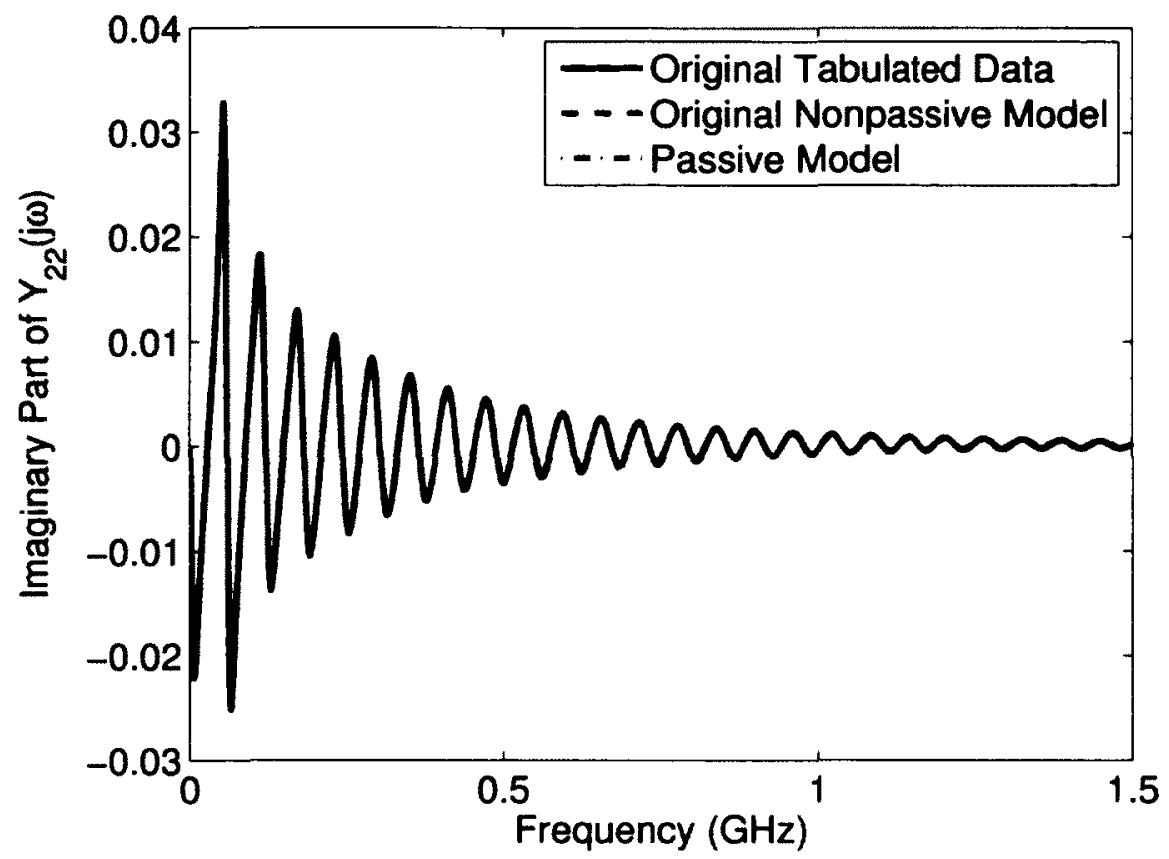

(a)

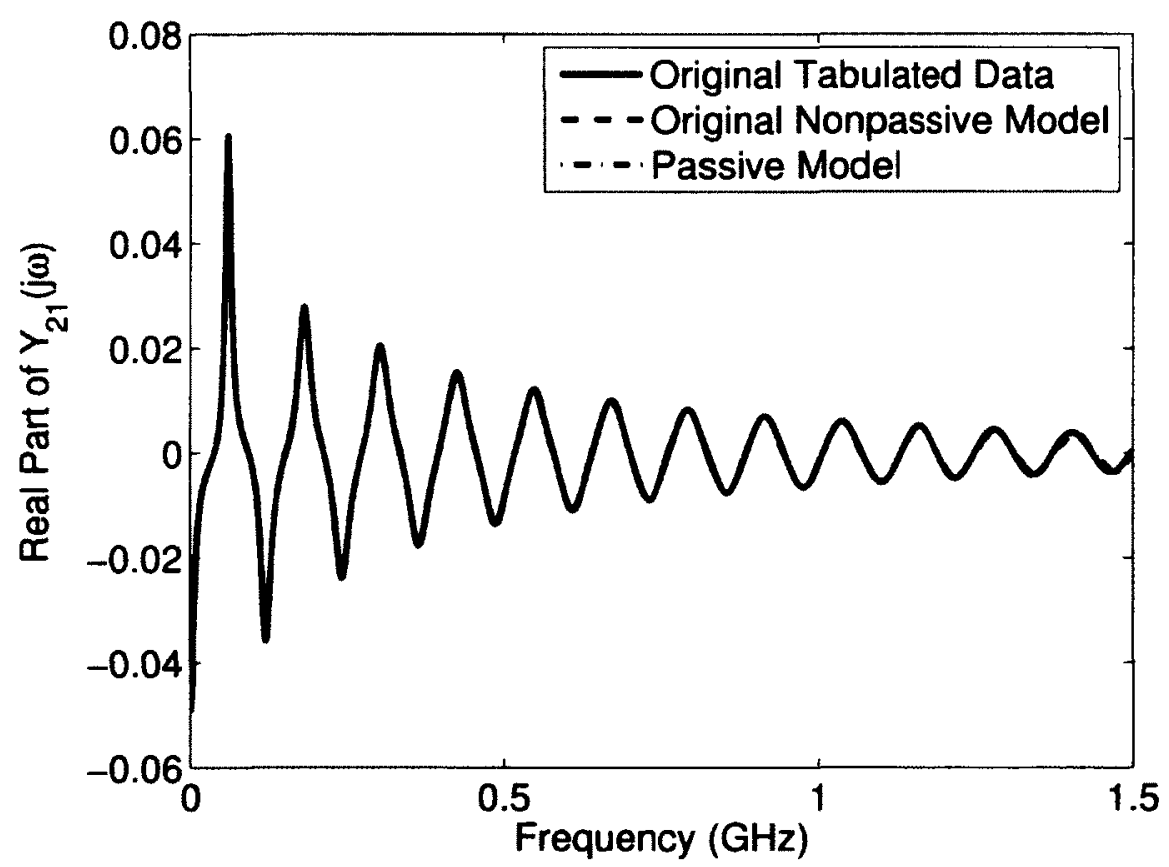

(b)

FIGURE 5.9: Accuracy of the DRF macromodel for example 1 (the Original Tabulated Data, Original Nonpassive Model, and Passive Model are overlapping). 


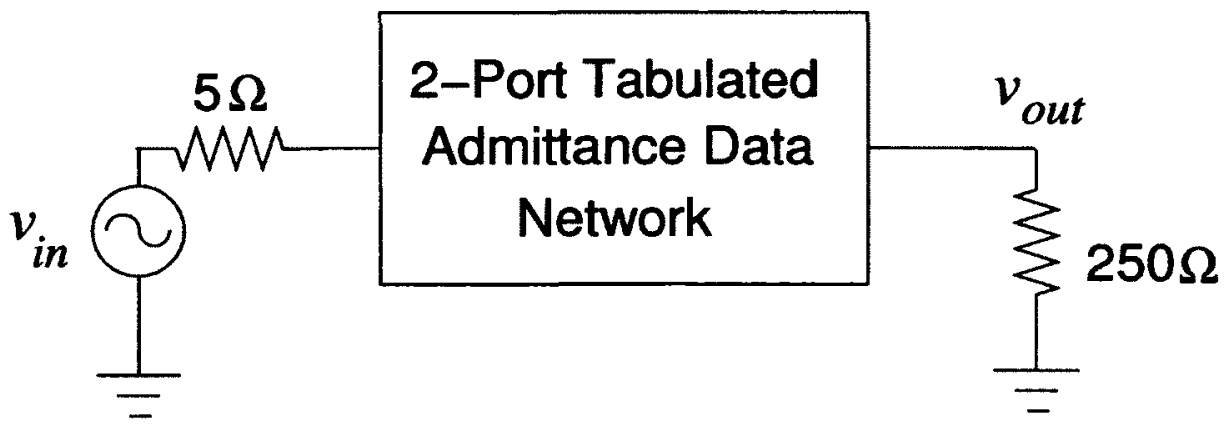

FIGURE 5.10: Circuit for transient analysis in example 1.

parameter was fit separately). A sample illustration showing the accuracy of the approximation is given in Fig. 5.12.

Next, the passivity of the DRF macromodel was checked. Solving the FDGEP in (5.16) the maximum pure imaginary eigenvalue was found to be $70.7851 \times 10^{9} \mathrm{rad} / \mathrm{s}$ [which corresponds to the maximum frequency of the passivity verification search region (i.e. $f_{\max }=70.7851 / 2 \pi=11.2658 \mathrm{GHz}$ ) ]. Fig. 5.13 shows the corresponding eigenvalue spectrum for the FDGEP. Using Theorem 5.3, the macromodel was found to be passive.

The above results were cross-verified by performing a robust search of the eigenvalues of $\left[\boldsymbol{Y}^{H}(j \omega)+\boldsymbol{Y}(j \omega)\right]$ along the imaginary axis. No negative eigenvalues of $\left[\boldsymbol{Y}^{H}(j \omega)+\boldsymbol{Y}(j \omega)\right]$ were found, confirming that the macromodel is passive. Fig. 5.14 shows a plot of the eigenvalues for $\left[\boldsymbol{Y}^{H}(j \omega)+\boldsymbol{Y}(j \omega)\right]$ between 0 and $11.3 \mathrm{GHz}$. It is clear from the figure that this model is indeed passive.

\subsubsection{Example 3}

In this example, the proposed algorithm was applied to a four-port telecommunication switching circuit link shown in Fig. 5.15 (example provided by Celestica Inc., Ottawa, ON, Canada). The interconnecting link extends across a backplane connecting the 


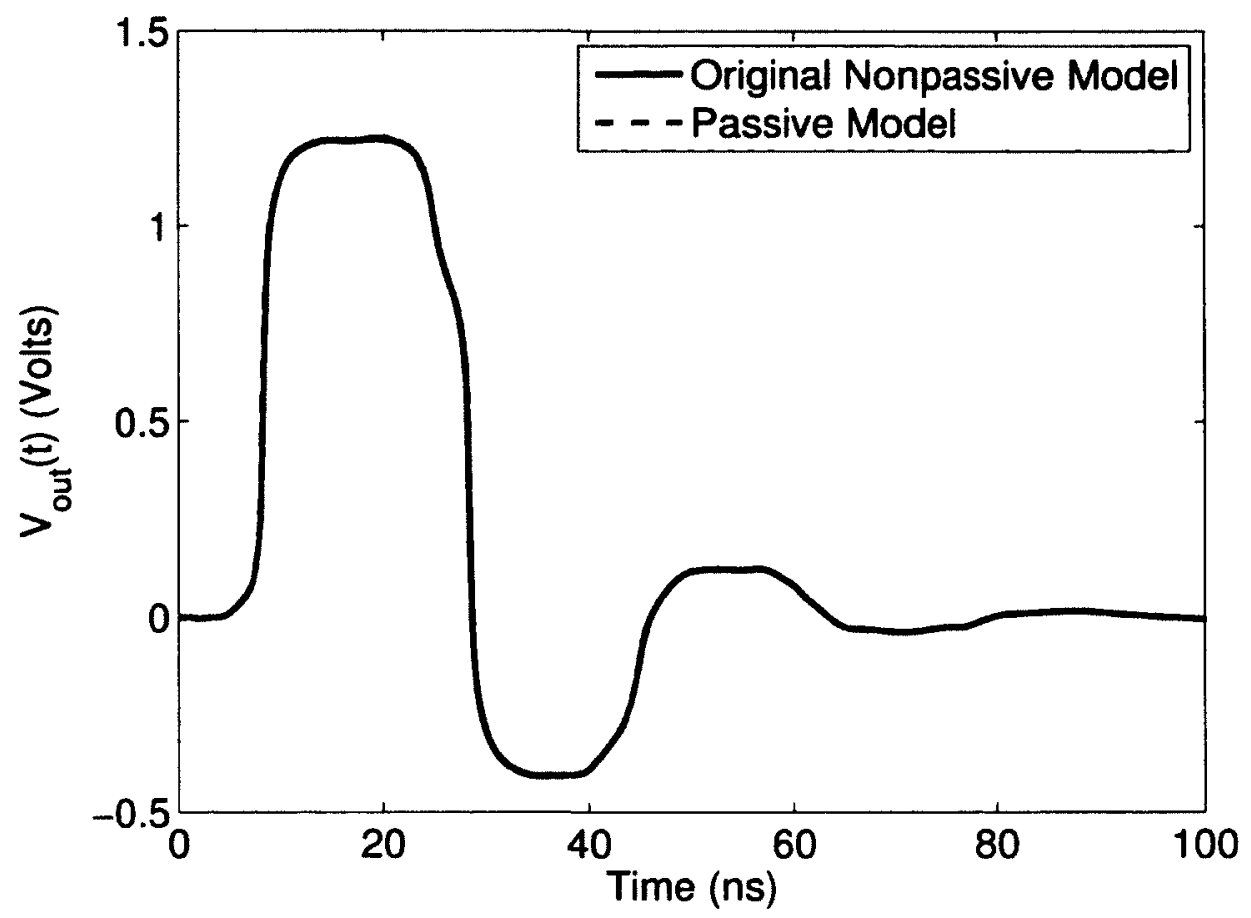

FIGURE 5.11: Transient response for $V_{\text {out }}(t)$ in example 1 (the Original and Passive Model are overlapping).

fabric manager on the line card and the switching devices on the fabric card. Using the delayed vector fitting algorithm [30], a total of 320 poles and 11 delays were required (each parameter was fit separately) to obtain an accurate approximation of the data up to $2.5 \mathrm{GHz}$.

Next, passivity verification of the DRF macromodel was performed. Solving the FDGEP in (5.16) the maximum pure imaginary eigenvalue was found to be $23.4991 \times$ $10^{9} \mathrm{rad} / \mathrm{s}$ [which corresponds to the maximum frequency of the passivity verification search region (i.e. $f_{\max }=23.4991 / 2 \pi=3.7400 \mathrm{GHz}$ ) ]. Fig. 5.16 shows the corresponding eigenvalue spectrum of the FDGEP. Then, using Theorem 5.3 the macromodel was found to be nonpassive along the imaginary axis on the intervals $[0,0.7870] \times 10^{-9} \mathrm{GHz}$ and $[0.32283,2.5543] \times 10^{-3} \mathrm{GHz}$. 


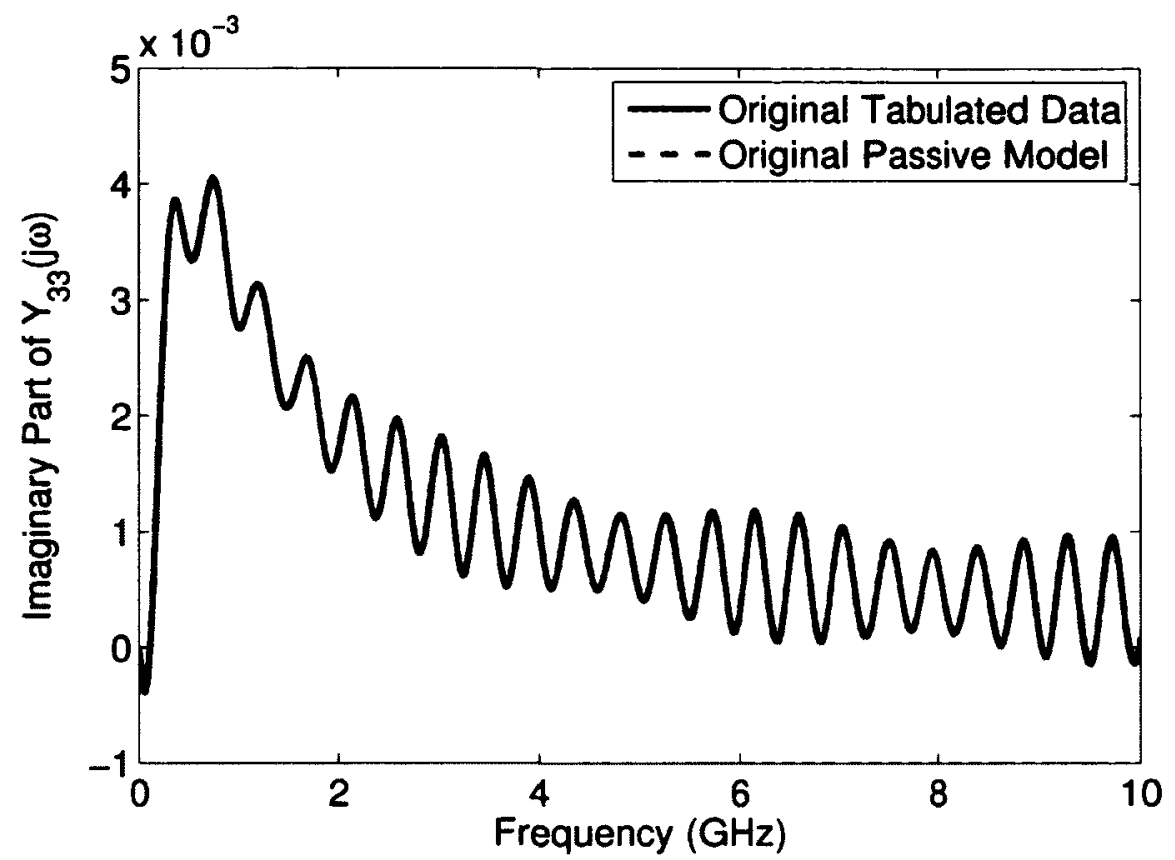

(a)

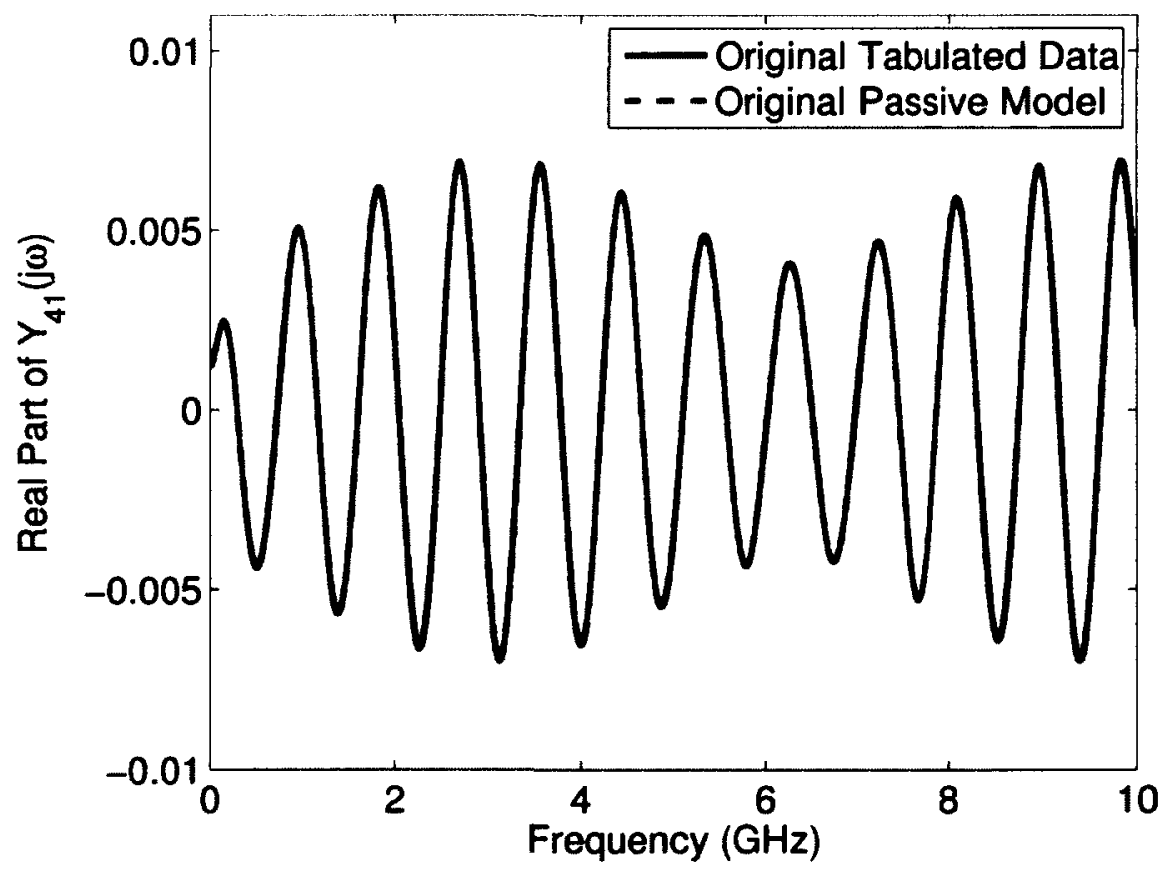

(b)

FIGURE 5.12: Accuracy of the DRF macromodel for example 2 (the Original Tabulated Data and Original Passive Model are overlapping). 


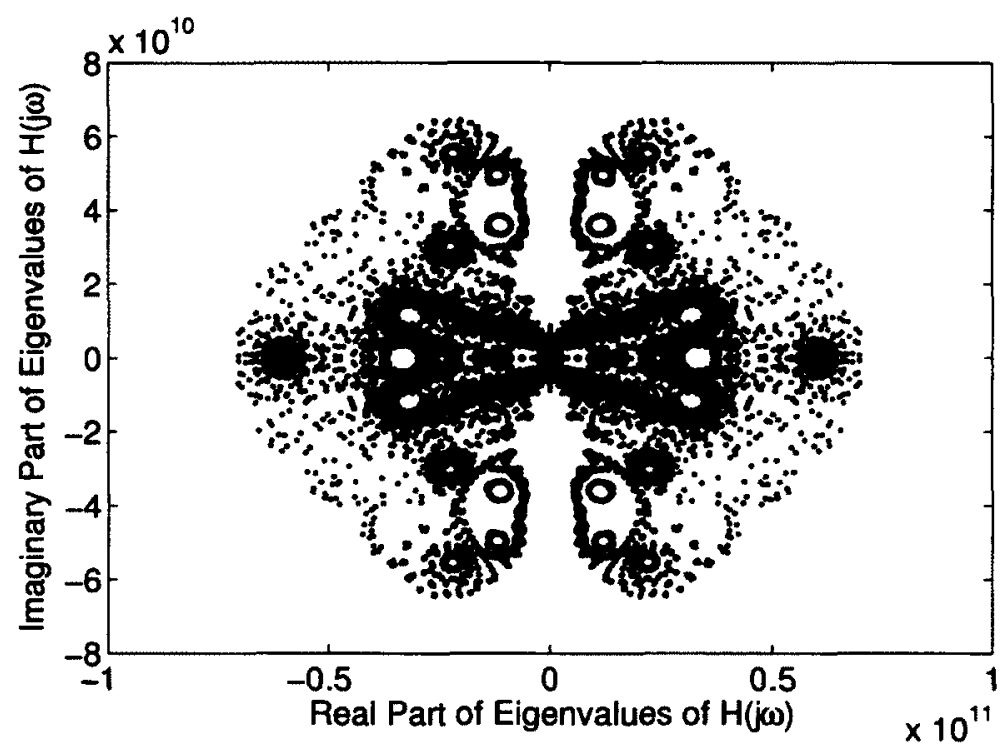

FIGURE 5.13: Eigenvalue spectrum of the FDGEP for example 2.

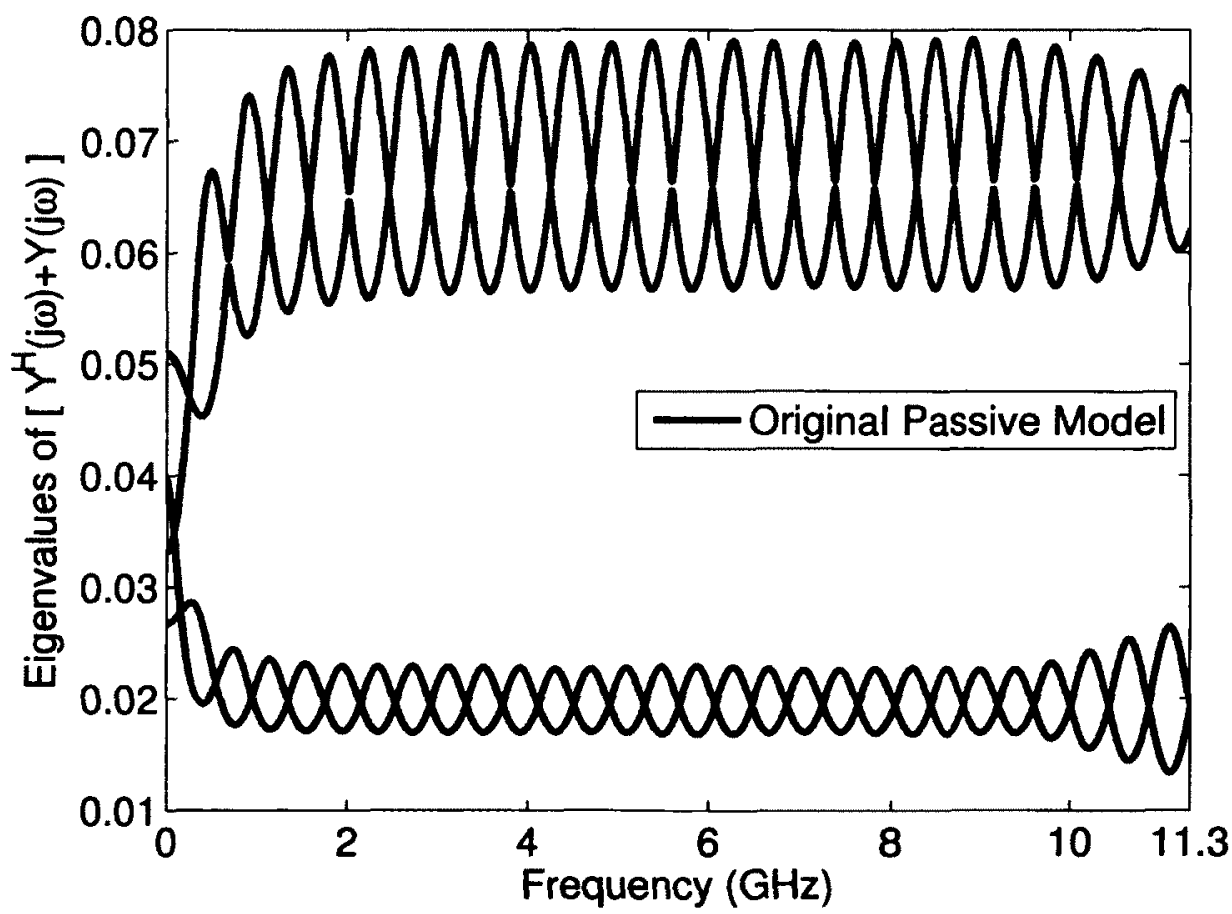

FIGURE 5.14: Real eigenvalues of $\left[\boldsymbol{Y}^{H}(j \omega)+\boldsymbol{Y}(j \omega)\right]$ for example 2 . 


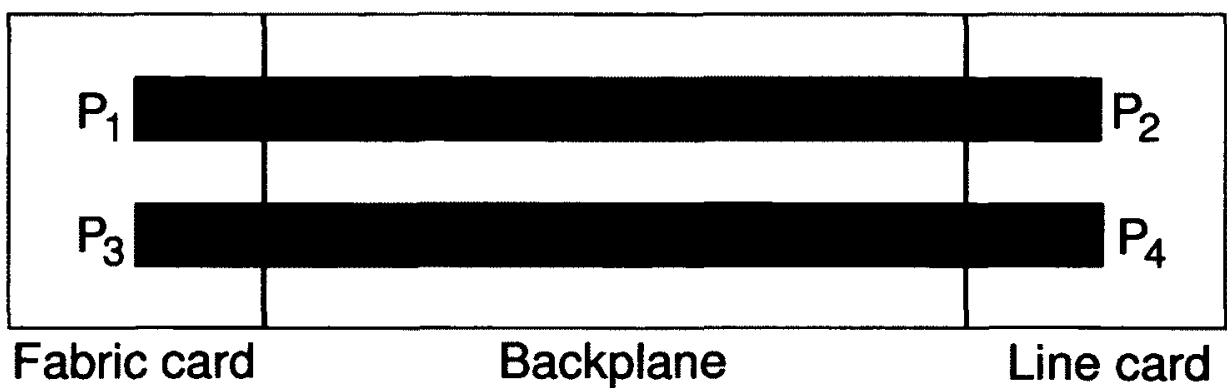

FIGURE 5.15: Telecommunication switching circuit link for example 3 .

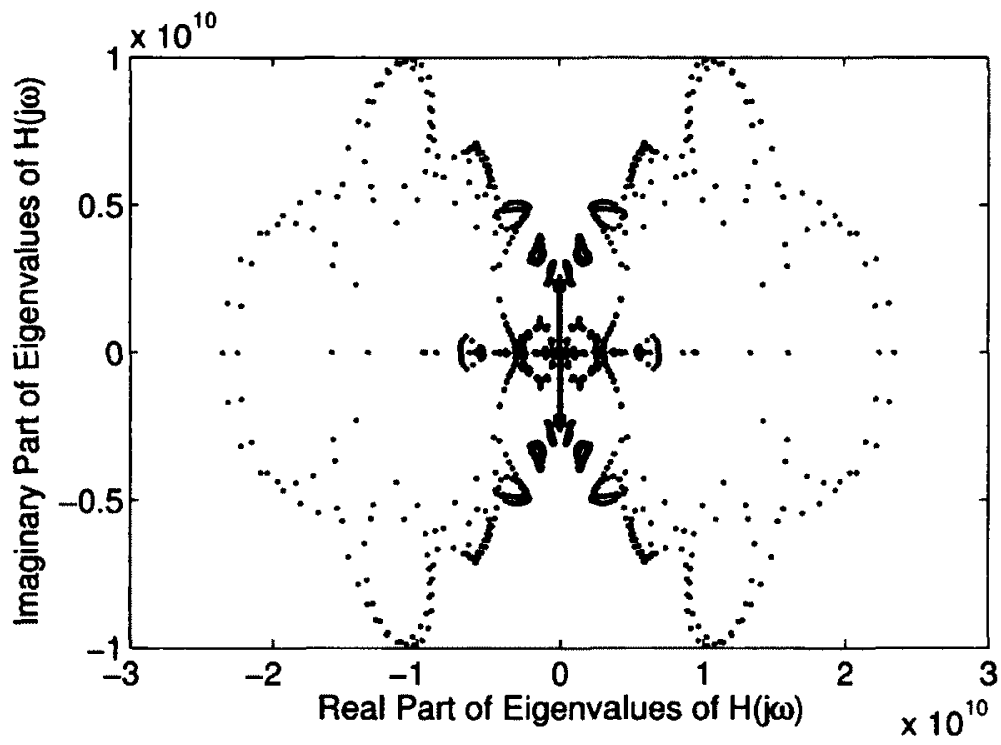

FIGURE 5.16: Eigenvalue spectrum of the FDGEP for example 3.

To cross-verify the accuracy of the above results, a robust frequency-sweep of the eigenvalues of $\left[\boldsymbol{Y}^{H}(j \omega)+\boldsymbol{Y}(j \omega)\right]$ was performed. Fig. 5.17 plots the results of the eigenvalues. It is clear from Fig. 5.17(b) that the macromodel is nonpassive on the interval $[0.32283,2.5543] \times 10^{-3} \mathrm{GHz}$. The expanded view given in Fig. 5.18(a) verifies the macromodel is also nonpassive on the interval $[0,0.7870] \times 10^{-9} \mathrm{GHz}$, in agreement with the proposed algorithm.

To enforce passivity in the DRF macromodel, the proposed passivity compensation algorithm is applied. From Fig. 5.17(b) and 5.18(b), it is clear that the eigenvalues 


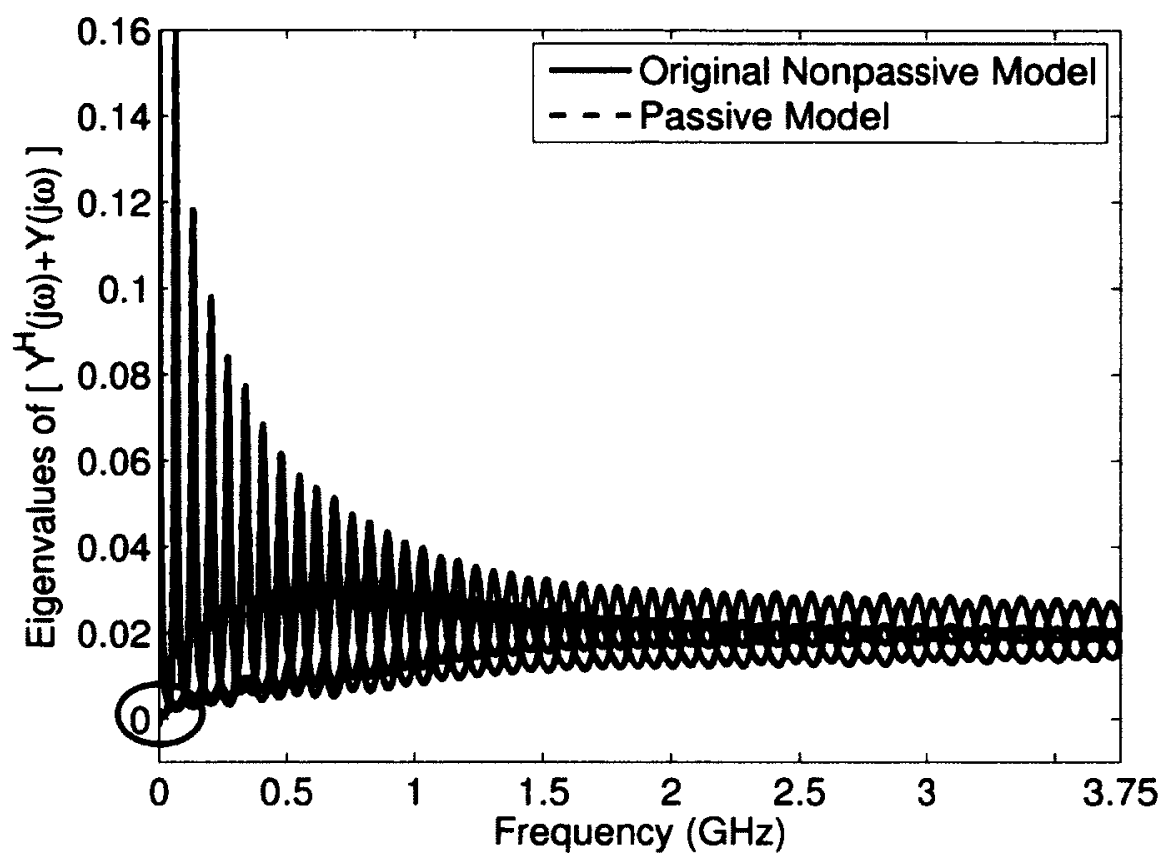

(a) Eigenvalue spectrum of the macromodels between 0 and $3.75 \mathrm{GHz}$ (the Original and Passive Model are overlapping).

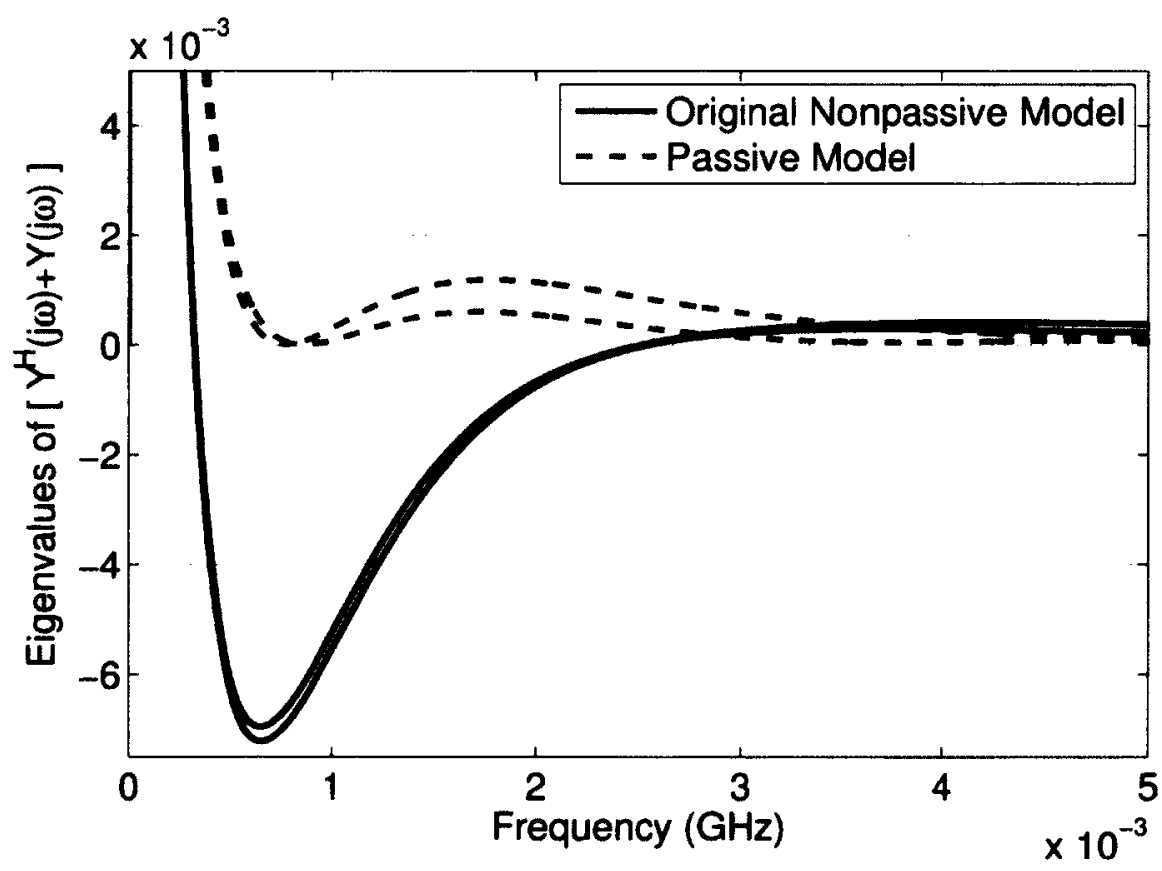

(b) Expanded view between 0 and $0.005 \mathrm{GHz}$.

FIGURE 5.17: Real eigenvalues of $\left[\boldsymbol{Y}^{H}(j \omega)+\boldsymbol{Y}(j \omega)\right]$ for example 3 . 


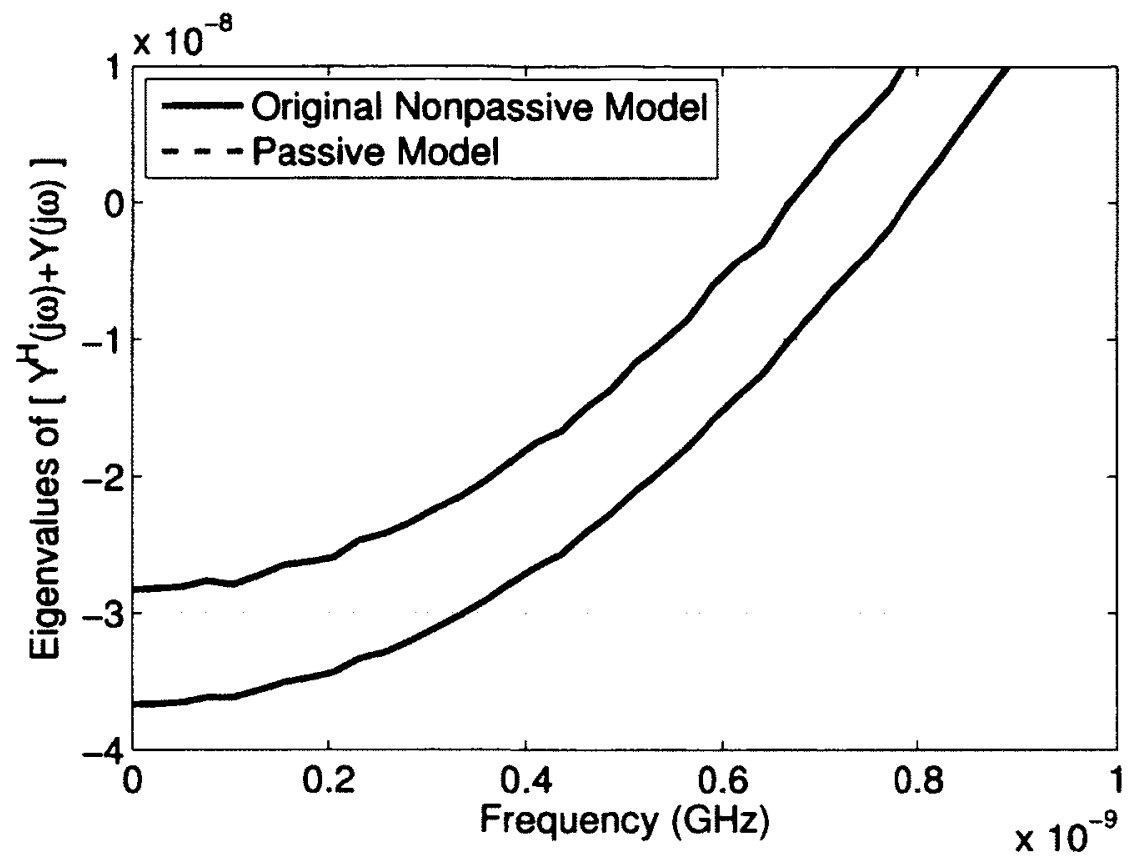

(a) Expanded view of eigenvalues for the original nonpassive model (passive model out of range).

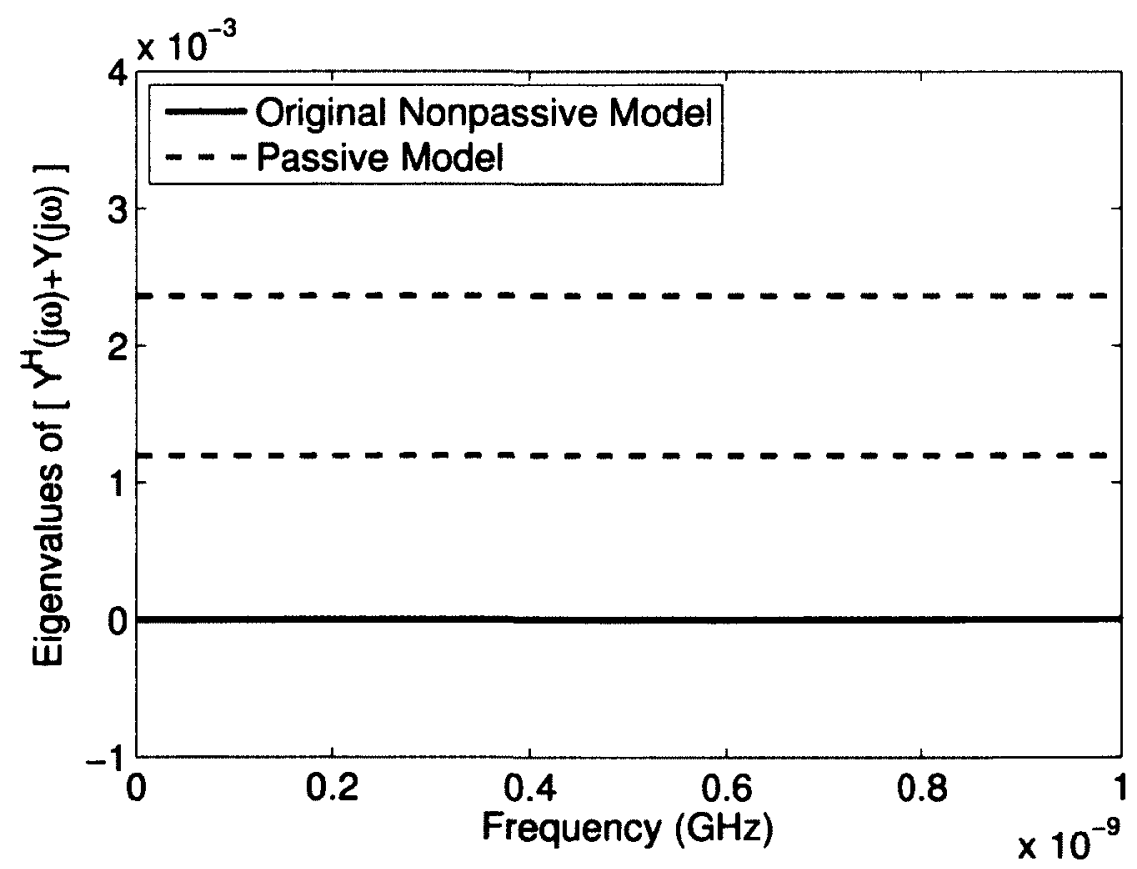

(b) Expanded view of eigenvalues for the passive model.

FIGURE 5.18: Expanded view of eigenvalues for example 3 on the interval $[0,0.7870] \times 10^{-9} \mathbf{G H z}$. 


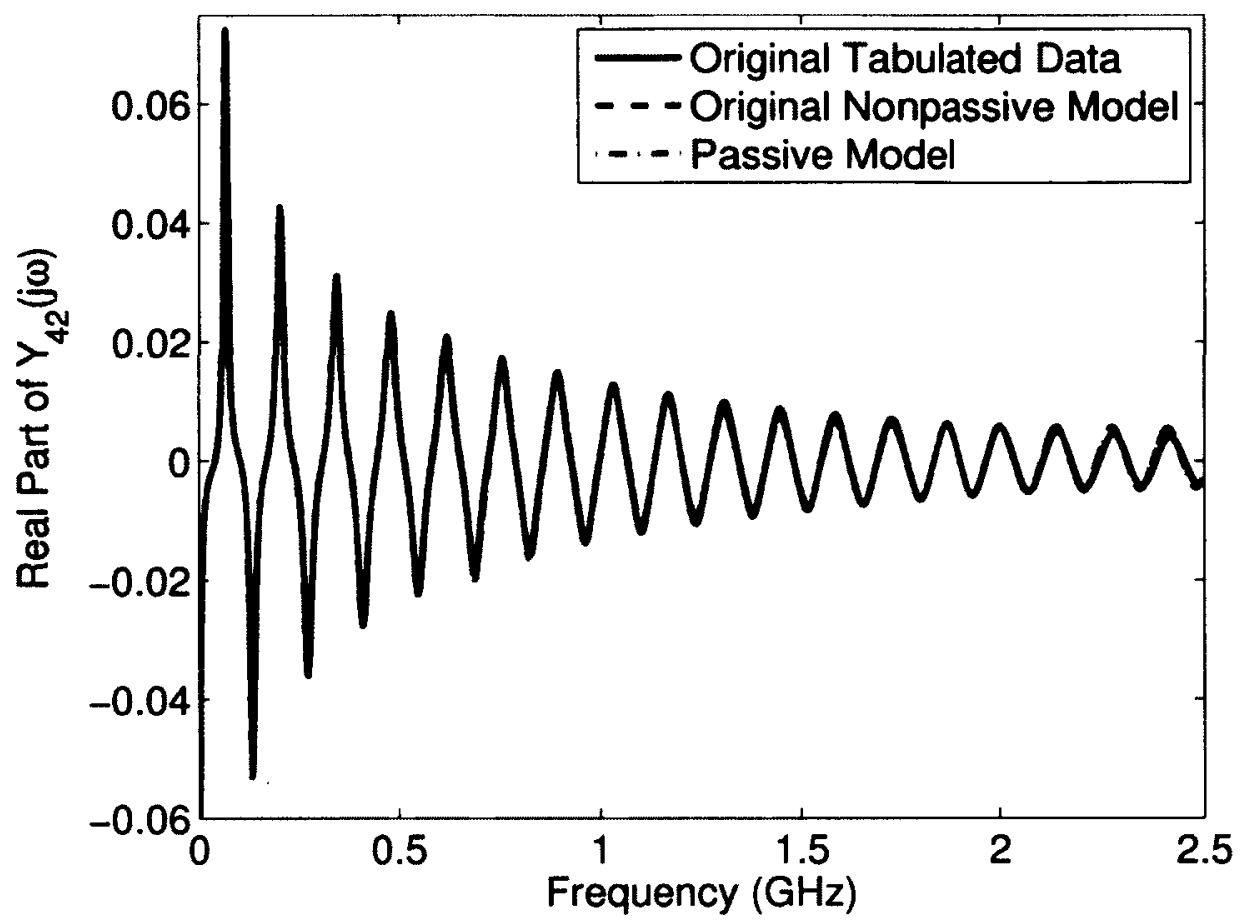

FIGURE 5.19: An illustration of the DRF approximation for example 3 (the Original Tabulated Data, Original Nonpassive Model, and Passive Model are all overlapping).

of $\left[\boldsymbol{Y}^{H}(j \omega)+\boldsymbol{Y}(j \omega)\right]$ for the compensated model are no longer negative. Applying the proposed passivity verification algorithm to the compensated macromodel, it was verified to be passive. In addition, a sample comparison of the admittance parameters for the original and compensated DRF macromodels is given in Fig. 5.19. It is clear from the figure that the frequency-domain responses for both models are in agreement.

Next, the accuracy of the passive, compensated DRF macromodel is cross-verified in the time-domain. The original and compensated DRF macromodels were connected to the terminations shown in Fig. 5.20. Then, each circuit was excited using a trapezoidal input signal with a $15 \mathrm{~ns}$ wide pulse and $0.1 \mathrm{~ns}$ rise/fall times. A comparison of the transient responses at the output node $V_{\text {out }}(t)$ is given in Fig. 5.21. As expected, the original and compensated DRF macromodels are in good agreement. 


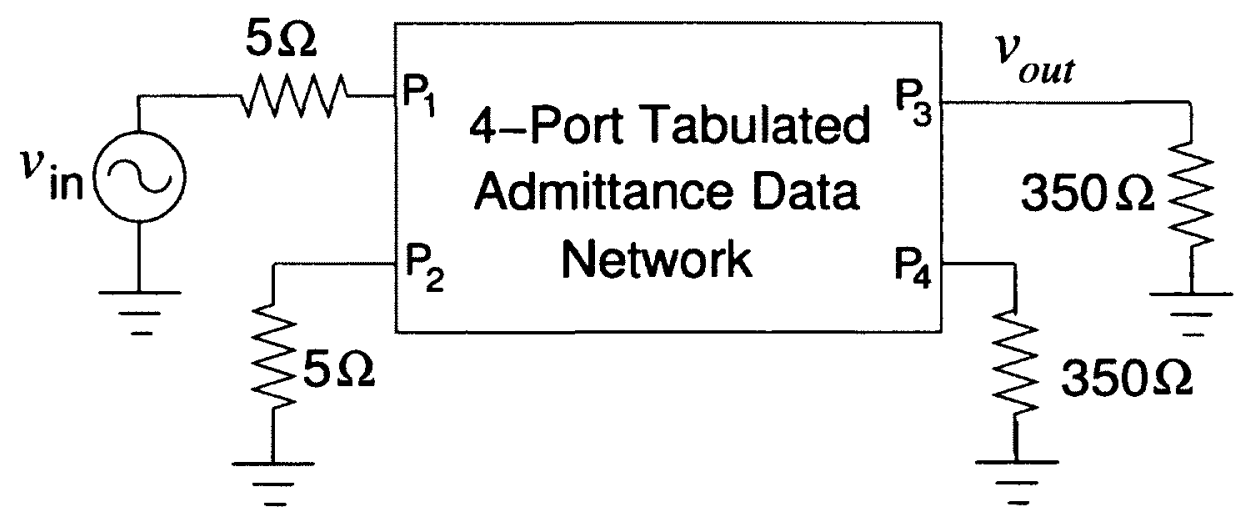

FIGURE 5.20: Circuit for transient analysis in example 3.

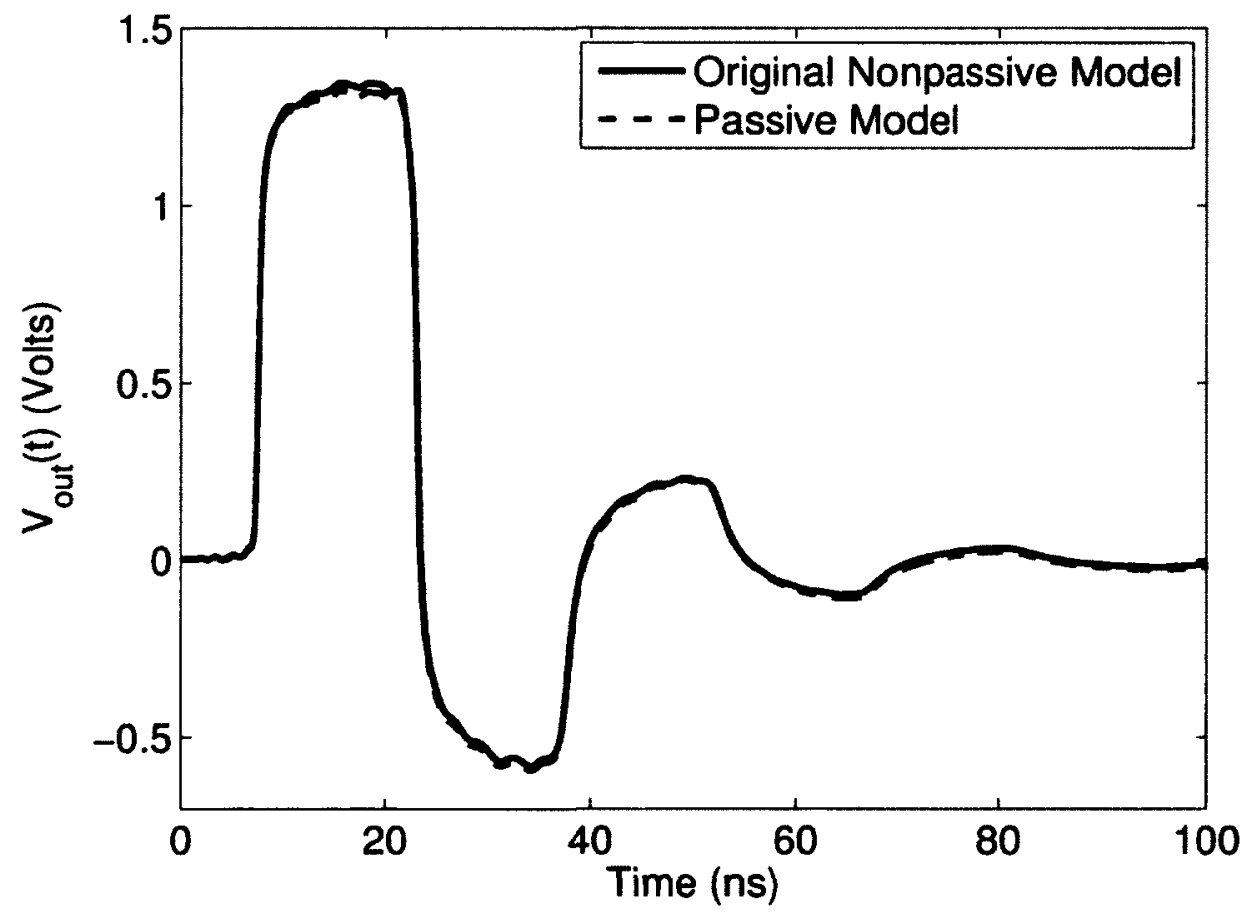

FIGURE 5.21: Transient response for $V_{\text {out }}(t)$ in example 3 . 


\subsection{Summary}

In this chapter, robust and efficient passivity verification and compensation algorithms were developed for DRF macromodels obtained from tabulated admittance data. Passivity verification was formulated as a quasi-periodic FDGEP, which reduced the necessary search region from the entire right-half of the complex plane to a single, finite interval along the imaginary axis. For passivity compensation, a scheme based on perturbing the residue matrices using linear matrix inequalities was presented. Detailed proofs of all necessary lemmas and theorems were given. Several practical examples validating the effectiveness and accuracy of the proposed passivity verification and enforcement techniques were also presented. 


\section{CHAPTER 6}

\section{Stable Periodic Delayed Rational \\ Function Based Macromodels for}

\section{Tabulated Data Networks}

In this chapter, a new efficient and stable macromodeling formulation for electrically long high-speed modules characterized by tabulated data is presented. The chapter is organized as follows. Section 6.1 introduces the topic and provides motivation for the new method. Section 6.2 discusses delayed rational function formulations for networks with periodic reflections. Section 6.3 provides details of the proposed macromodeling algorithm and is followed by Section 6.4, which develops a framework for stability verification and enforcement. Numerical results validating the efficiency of the proposed technique are presented in Section 6.5.

The work in this chapter first appeared in [72]. 


\subsection{Introduction}

As discussed in Chapter 1, several compact and efficient macromodeling algorithms for tabulated networks with long delays have recently been introduced [26]-[34], [54]-[60]. In [31]-[34], Hilbert transform based delay estimation was proposed for networks characterized by tabulated frequency-domain data. In [28], a Gabor transform based macromodeling technique was proposed and [29], [30] extended this technique to be formulated via delayed vector fitting. The work in [55] (applicable to transmission line type modules) incorporates delay estimation into the vector fitting algorithm using optimization. Multiple delays are taken into account via the modal decomposition of the propagation function matrix. In [26], [27], general delayed rational function based macromodeling techniques were developed for networks characterized by tabulated time-domain data. Conceptually, algorithms in [26]-[30] approximate each reflection in the response using a separate delayed rational term. Although the above approaches provide a reduction in the approximation order for networks with long delays, they are not optimal for systems that contain periodic reflections. To address this issue, in [56], [57] a transmission line specific delay embedded passive compact macromodel was developed to handle infinite reflections in tabulated data. In [59], [60], a formulation tackling a similar problem was presented. However, it is also limited to transmission lines and does not provide a method to guarantee the stability of the overall macromodel.

In this chapter, a generalized formulation of [59], [60] is developed for general highspeed modules characterized by tabulated data with multiple delays. The proposed algorithm more efficiently handles dominant periodic delays. Consequently, the resulting system of delay differential equations contains a reduced number of delayed 
rational terms leading to faster transient simulations. In addition, stability verification and enforcement methods for the proposed model are developed.

\subsection{Problem Formulation and Review}

Let the scattering equations of a 2-port network be in the form $\boldsymbol{b}=\boldsymbol{S a}$, where $\boldsymbol{a}$ and $\boldsymbol{b}$ are the incident and reflected waves, respectively, and $\boldsymbol{S}(s)=\left[S_{i k}(s)\right], i, k \in$ $\{1,2\}$ represents the scattering matrix. For macromodeling networks with long delays, $S_{i k}(s)$ is approximated with a delayed rational function in the form

$$
S_{i k}(s)=\Phi_{0}^{i, k}(s)+\sum_{m=1}^{M} \Phi_{m}^{i, k}(s) e^{-s T_{m}}
$$

where $\left\{T_{m}\right\}$ are the time-delays and $\Phi_{m}^{i, k}(s)$ are the rational functions expressed in terms of poles and residues.

However, for networks with a large number of reflections, the above formulation can still be inefficient (requiring large order due to the summation of many delayed rational approximations). For the purpose of illustration, consider the two-port network shown in Fig. 6.1(a) with a port-to-port delay $\tau / 2$. The response at port 1, due to an input excitation at port 1 can be represented by the closed-loop feedback control system in Fig. 6.1(b). The complete transfer function at port 1, containing all reflections for an input at port 1 is then given by

$$
\begin{aligned}
S_{11}(s)= & {\left[Q_{0}(s)+Q_{1}(s) e^{-s \tau}\right]+\left[Q_{0}(s)+Q_{1}(s) e^{-s \tau}\right] Q_{1}(s) Q_{2}(s) e^{(-s \tau)} } \\
& +\left[Q_{0}(s)+Q_{1}(s) e^{-s \tau}\right] Q_{1}^{2}(s) Q_{2}^{2}(s) e^{(-2 s \tau)}+\ldots
\end{aligned}
$$




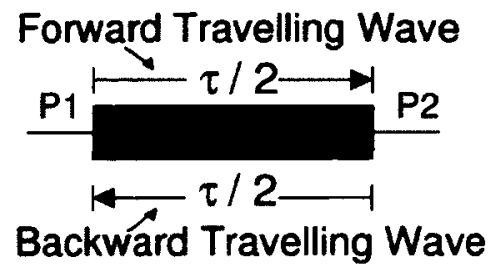

(a) Two-port network.

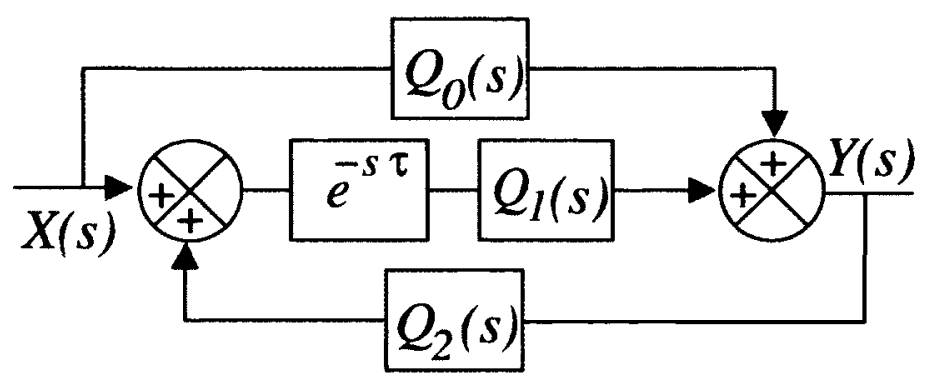

(b) Closed-loop feedback control system characterizing $S_{11}(s)$, for the network in Fig. 6.1(a).

FIGURE 6.1: Example of a system with multiple periodic delayed responses from an internal reflection.

where $Q_{i}(s)$ are the transfer functions of the signal as it travels through the network. As seen from (6.2), the above network requires a new term in summation for each reflected response if it were described by (6.1), leading to a large order for the macromodel. Therefore, it is desired to find a more compact representation of (6.2).

Noting that (6.2) is equivalent to a Taylor series expansion of the function $1 /(1-$ $x) \approx 1+x+x^{2}+x^{3}+\ldots$, at $x=0$, it can be expressed as

$$
S_{11}(s)=\frac{Q_{0}(s)+e^{-s \tau} Q_{1}(s)}{1-Q_{1}(s) Q_{2}(s) e^{(-s \tau)}}
$$

The transfer functions $Q_{0}(s), Q_{1}(s)$, and $Q_{2}(s)$ can now be approximated by loworder pole/residue approximations (since the delay has already been extracted). A similar result can be obtained for other entries in the $\boldsymbol{S}$ matrix. 


\subsection{Construction of Periodic Delayed Rational}

\section{Functions}

The formulation in (6.3) is limited to transmission lines with only a single delay considered. However, circuits in general will contain more than a single periodic delayed response. In order to overcome this issue, the numerator in (6.3) is replaced with (6.1) to obtain a general form

$$
\hat{S}(s)=\frac{\Phi_{1}(s)+\sum_{m=2}^{M} \Phi_{m}(s) e^{-s \kappa_{m}}}{1+\Phi_{0}(s) e^{-s \tau}}
$$

where $\Phi_{i}(s),(i=0,1, \ldots, M)$, are approximated with low-order rational functions. Here, $\tau$ corresponds to the dominant periodic reflective delay in the network, while the delays $\left\{\kappa_{i}\right\}$ correspond to each of the unique delayed responses. In the following section, details of the proposed algorithm are given.

\section{Determination of $\tau$ and $\left\{\kappa_{i}\right\}$}

For determination of the periodic delay $\tau$ and the delays $\left\{\kappa_{i}\right\}$, the following algorithm is developed. First, a time-frequency analysis [26], [28] of an off diagonal element (say $\left(S_{12}(s)\right)$ is performed and the set of all the corresponding delays, $\left\{T^{\{1,2\}}\right\}=\left\{T_{1}^{\{1,2\}}<\right.$ $\left.T_{2}^{\{1,2\}}<\ldots<T_{M}^{\{1,2\}}\right\}$, is identified. The dominant reflective delay, $\tau$, is chosen as 2 times the port-to-port delay, $T_{1}^{\{1,2\}}$. Subsequently, the delays $\left\{\kappa^{\{1,2\}}\right\}$ are found by peeling all delays from the set $\left\{T^{\{1,2\}}\right\}$ that occur at $\left[T_{1}^{\{1,2\}}+b_{12} \tau\right]$, where $b_{12}>0$ is an integer (for example, consider the set of delays $(1.2,3.6,4.1,6)$; here $\tau=1.2 * 2=2.4$; consequently, the delays $(3.6,6)$ are removed, thereby giving $\left.\left\{\kappa^{\{1,2\}}\right\}=(1.2,4.1)\right)$. Next, for the diagonal elements (say $S_{11}(s)$ ), the set of delays $\left\{\kappa^{\{1,1\}}\right\}$ is found by 
peeling all delays from the set $\left\{T^{\{1,1\}}\right\}$ that occur at $\left[T_{1}^{\{1,1\}}+b_{11} \tau\right]$, where $b_{11}>1$ is an integer.

\section{Computation of Poles and Residues of (6.4)}

For the purpose of calculating the poles and residues in (6.4), we assume that the delays $\tau$ and $\left\{\kappa_{i}\right\}$ are already computed. Note that $\hat{S}(s)$ in (6.4) can be written as

$$
\hat{S}(s)=\Phi_{1}(s)+\sum_{m=2}^{M} \Phi_{m}(s) e^{-s \kappa_{m}}-\hat{S}(s) \Phi_{0}(s) e^{-s \tau}
$$

Next, a rational approximation of the form

$$
\Phi_{m}(s)=\frac{R_{m, 0}+\sum_{n=1}^{N} \frac{R_{m, n}}{s-p_{n}}}{r_{0}+\sum_{n=1}^{N} \frac{r_{n}}{s-p_{n}}}
$$

for each of the $\Phi_{m}(s)$ is substituted into (6.5). Subsequently, following the steps outlined in [59], [60], (6.6) can be solved iteratively as a linear least squares problem for the residues $\left\{r_{n}\right\},\left\{R_{m, n}\right\}$ and the poles $\left\{p_{n}\right\}$.

The above formulated macromodel is more compact than macromodels characterizing equations in the form of (6.1) in cases where a dominant internal reflection results in multiple periodic delayed responses. The next section discusses the stability of the proposed macromodel. 


\subsection{Verification and Enforcement of Stability}

For stable rational approximations it is required that all poles lie in the left-half plane. For stability verification of (6.4), the following Lemma is developed.

Lemma 6.1: If $A(s), B(s), C(s), D(s)$ are real polynomials (contain only real coefficients), then any unstable pole in the following function

$$
H(s)=\frac{\frac{A(s)}{C(s)}+\frac{B(s)}{C(s)} e^{-s \tau}}{1+e^{-s \tau} \frac{D(s)}{C(s)}}
$$

occurs when $|T(s)|=|D(s) / C(s)|=1$ provided the rational function $T(s)$ is bounded real.

Proof of Lemma 6.1: To begin, we assume $T(s)=D(s) / C(s)$ is a bounded real rational function. Then, the roots of $C(s)$ are all in the left-half plane. Consequently, the poles that arise from the numerator terms $A(s) / C(s)$ and $B(s) / C(s) e^{-s \tau}$ are all stable.

Next, consider the term in the denominator, $\left[1+e^{-s \tau} T(s)\right]$, where the poles occur when $e^{-s \tau} T(s)=-1$. Applying the Cauchy-Schwarz inequality to the left hand side, noting that for $\operatorname{Re}(s)>0,\left|e^{-s \tau}\right| \leq 1$, and using the fact that for a bounded real function $\boldsymbol{\Psi}(s),\left[\boldsymbol{I}-\boldsymbol{\Psi}^{H}(s) \Psi(s)\right] \geq 0$, we get

$$
\left|e^{-s \tau} T(s)\right| \leq\left|e^{-s \tau}\right||T(s)| \leq|T(s)|=\sqrt{T^{H}(s) T(s)} \leq 1
$$

It is clear that $\left|e^{-s \tau} T(s)\right|=1$ only if $|T(s)|=1$ and this completes the proof.

Lemma 6.1 is extended to the form of (6.4) (i.e. for cases with multiple delays in the numerator) by noting that the additional numerator terms are all stable since the 
roots of $C(s)$ lie in the left-half plane while the denominator remains unaffected.

Based on Lemma 6.1, if $C(s)$ has all its roots in the left-half plane, then $H(s)$ has only unstable poles where $T^{H}(s) T(s)=1$. These locations can be easily found using the bounded real property and Hamiltonian based techniques [41].

Using the above properties, stability can be enforced by adding the following additional steps to the proposed algorithm. First, if the roots of $C(s)$ are found to lie in the right-half plane, they are flipped to the left-half plane and a new set of residues is calculated. Next, we apply first-order perturbation based compensation techniques [41] to the function $D(s) / C(s)$ to ensure that it is a bounded real rational function.

It is to be noted that the above algorithm can be extended by combining it with the technique presented in [50] to develop an efficient method for passivity verification and enforcement of the resulting macromodels.

\subsection{Numerical Results}

In this section, two examples are presented to demonstrate the accuracy and efficiency of transient analysis using the proposed delayed rational function macromodels for tabulated data networks with periodic reflections. All the examples are run on an Intel $i 7-9202.67 \mathrm{GHz}$ processor with $12 \mathrm{~Gb}$ of RAM.

\subsubsection{Example 1}

In this example, the proposed algorithm is tested using a set of tabulated admittance parameters characterizing a two-port network with multiple internal reflections. First, the proposed algorithm was applied to the tabulated data. Using the technique in [28], 
a time-frequency analysis was performed and based on the obtained results and the algorithm described in Section 6.3, the dominant reflective delay was found to be 15.74 ns ( 2 times the port-to-port delay of $7.87 \mathrm{~ns}$ ). Then, extracting the periodic delays the entire model required 36 poles and a total of 12 unique delays. To validate the proposed technique, it is compared to the conventional vector fitting algorithm [17] and the state-of-the-art delayed vector fitting algorithm [30]. Applying conventional vector fitting a total of 640 poles were required to accurately fit the entire data set. Applying the delayed vector fitting algorithm, a total of 49 poles and 16 delays were required to obtain an accurate approximation. A sample comparison for the frequency-domain fitting of $Y_{21}(s)$ using the proposed, delayed vector fitting [29], and conventional vector fitting against the original data is shown in Fig. 6.2(a). As seen, all 3 models provide a comparable approximation of the tabulated data. In addition, the corresponding absolute fitting error of $Y_{21}(s)$ for the proposed and delayed vector fitting is shown in Fig. 6.2(b). A comparison of the macromodel properties in terms of the number of required delay segments and poles, total number of pole/residue combinations used to fit each parameter, and Root Mean Squared Error (RMSE) from each approximation is given in Table 6.1.

Next, the accuracy and efficiency of the proposed macromodel is validated in the time-domain using HSPICE. For each macromodel, the circuit shown in Fig. 6.3 is stimulated with a $20 \mathrm{~ns}$ wide pulse that has rise/fall times of $0.1 \mathrm{~ns}$. A sample comparison of the transient responses for all three models is shown in Fig. 6.4. As seen, all the waveforms are in agreement. However, the proposed macromodel only required 0.92 seconds to simulate while the conventional vector fitting and delayed vector fitting macromodels required 11.01 and 1.74 seconds, respectively. The CPU times for each of the transient simulations were recorded and are shown in Table 


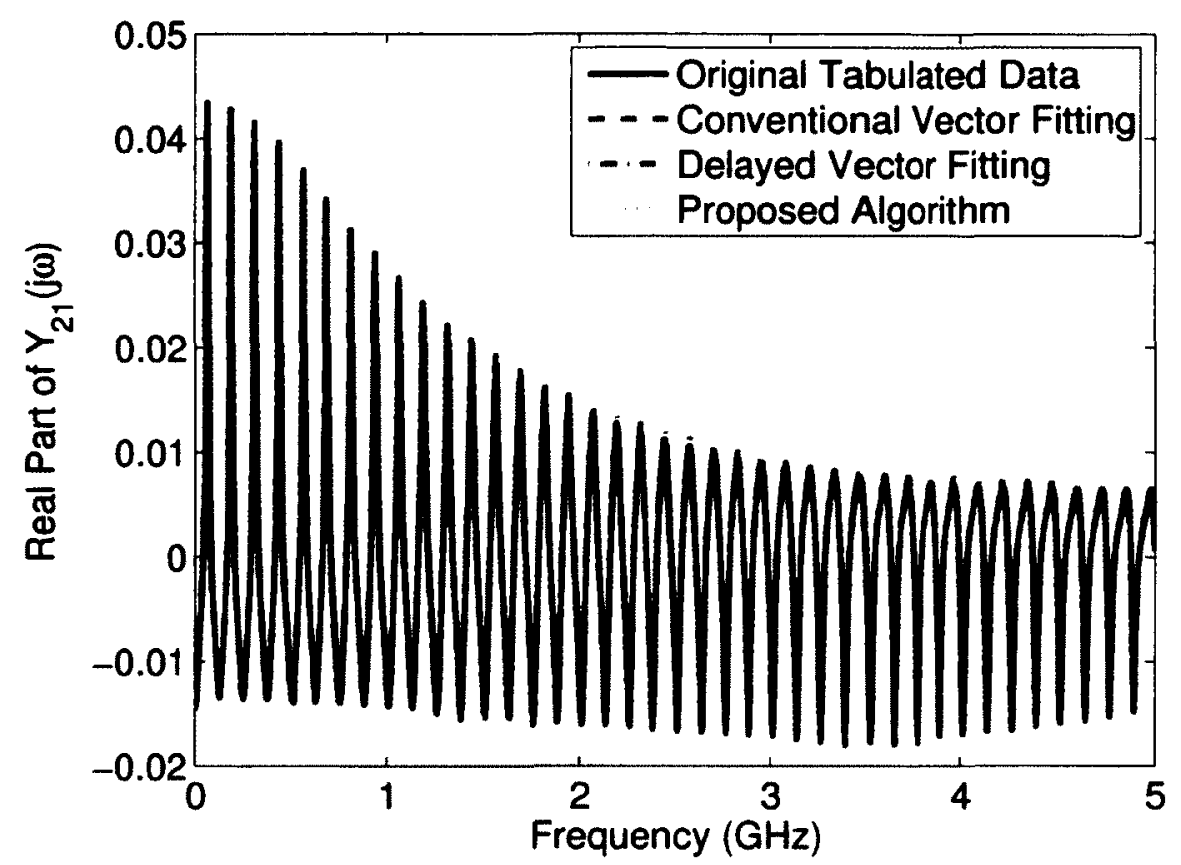

(a) Frequency-domain responses (all the responses overlap).

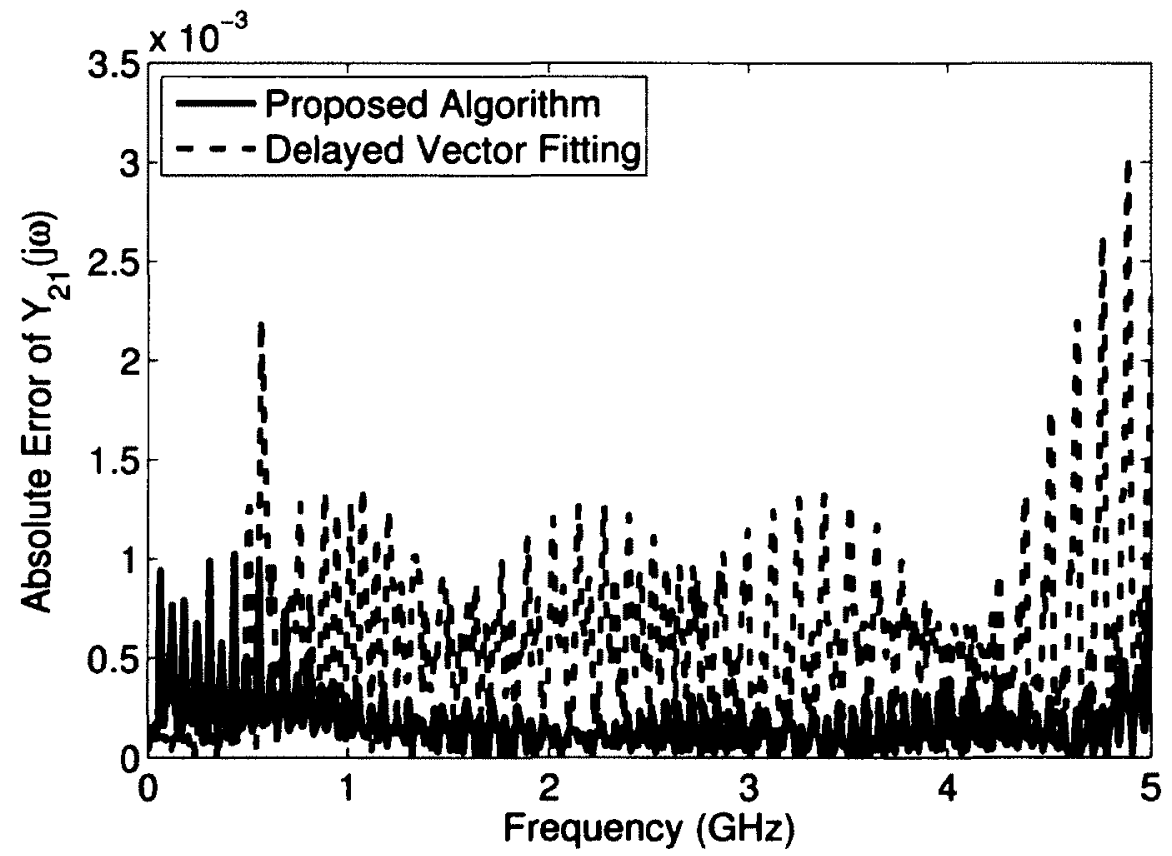

(b) Absolute error.

FIGURE 6.2: A sample comparison of the scattering parameters, $Y_{21}(j \omega)$, for example 1 . 
TABLE 6.1: A comparison of macromodel accuracy for example 1 (DRF: Delayed Rational Function; RMSE: Root Mean Squared Error).

\begin{tabular}{|c|c|c|c|}
\hline & Vector Fit & DRF ([29]) & Proposed \\
\hline $\begin{array}{l}Y_{11}(s) ; Y_{12}(s) \\
Y_{21}(s) ; Y_{22}(s)\end{array}$ & $\begin{array}{c}160 \\
\text { poles } \\
\text { for each } \\
\text { parameter } \\
\text { in the } Y \\
\text { matrix }\end{array}$ & $\begin{array}{l}11 \text { DRF terms } \\
\text { with } 9 \text { and } 12 \\
\text { poles each for } \\
Y_{11} \text { and } Y_{22} ; \\
10 \text { DRF terms } \\
\text { with } 14 \text { poles } \\
\text { each for } Y_{21}, Y_{12}\end{array}$ & $\begin{array}{c}6 \text { DRF terms } \\
\text { with } 9 \text { poles } \\
\text { each for } \\
Y_{11}, Y_{22} ; \\
7 \text { DRF terms } \\
\text { with } 9 \text { poles } \\
\text { each for } Y_{21}, Y_{12}\end{array}$ \\
\hline $\begin{array}{c}\text { Total Number of } \\
\text { Pole/Residue Terms }\end{array}$ & 640 & 511 & 234 \\
\hline RMSE & $7.1747 \times 10^{-5}$ & $6.2726 \times 10^{-4}$ & $2.7675 \times 10^{-4}$ \\
\hline Transient CPU Time (sec) & 11.01 & 1.74 & 0.92 \\
\hline
\end{tabular}

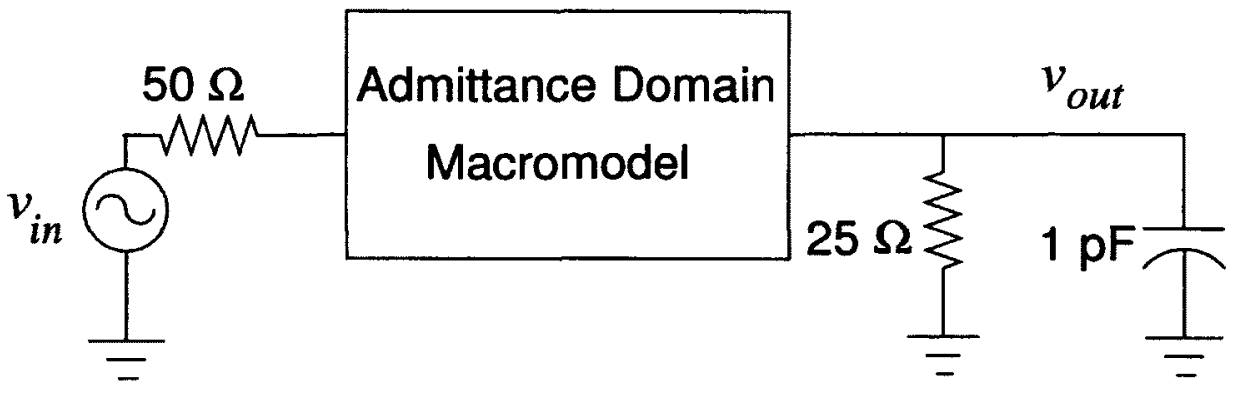

FIGURE 6.3: Circuit for transient analysis of example 1.

6.1. As seen, the proposed macromodel provides a speedup of 12 and 2 over the conventional vector fitting and delayed vector fitting macromodels, respectively.

\subsubsection{Example 2}

In this example, the proposed algorithm is applied to a set of tabulated scattering parameters characterizing a cascade of two 2-port networks (one containing a frequency-dependent stripline and the other a frequency-independent stripline with mismatched internal terminations) with multiple internal reflections. Using the tech- 


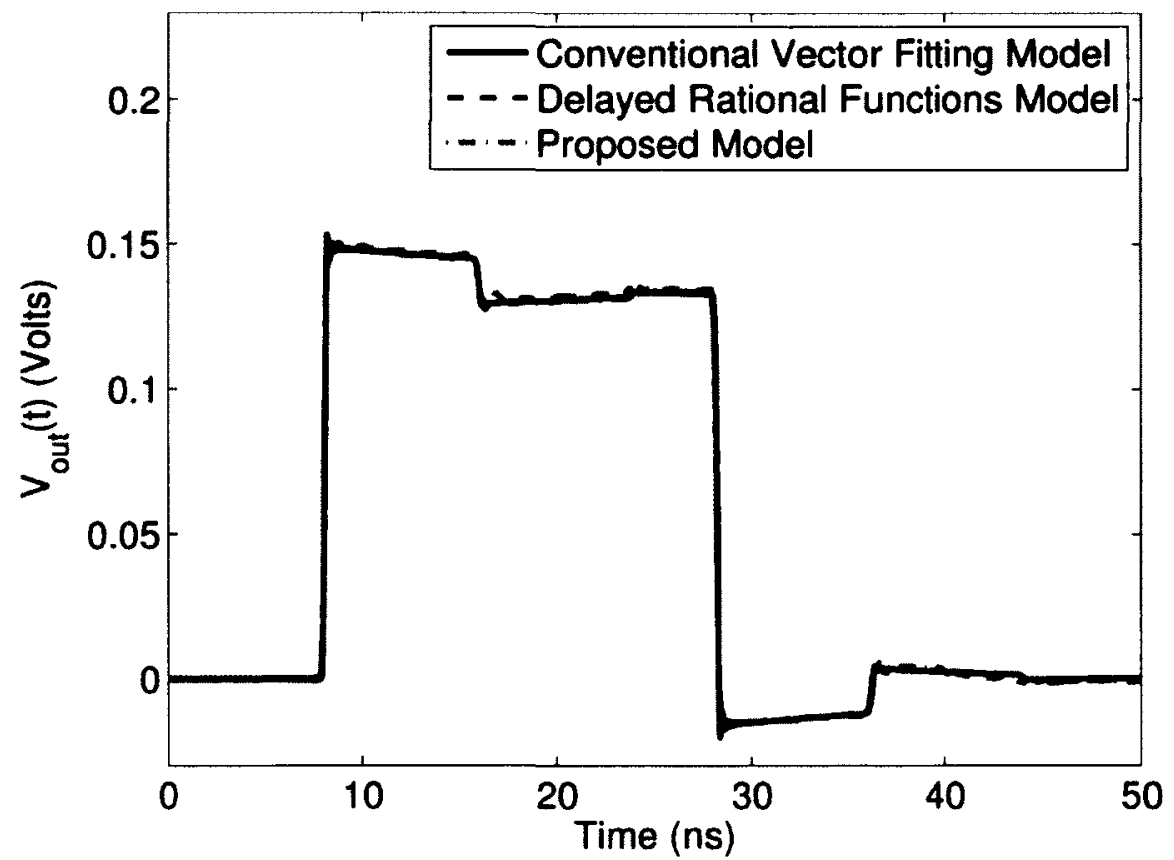

FIGURE 6.4: Comparison of transient responses for example 1 (all the responses overlap).

nique in [28], a time-frequency analysis was performed and based on the obtained results and the algorithm described in Section 6.3, the dominant reflective delay was found to be $21 \mathrm{~ns}$ ( 2 times the port-to-port delay of $10.5 \mathrm{~ns}$ ). A sample comparison for the frequency-domain fitting of $S_{22}(s)$ using the proposed, delayed vector fitting [29], and conventional vector fitting against the original data is shown in Fig. 6.5(a). In addition, the corresponding absolute fitting error of $S_{22}(s)$ for the proposed and delayed vector fitting is shown in Fig. 6.5(b). During the macromodel creation process, the original fitting was found to be unstable and the proposed stability enforcement technique was successfully applied to stabilize the macromodel. A comparison of the macromodel properties in terms of the number of required delay segments and poles, total number of pole/residue combinations used in the fit, and Root Mean Squared Error (RMSE) from each approximation is given in Table 6.2. 


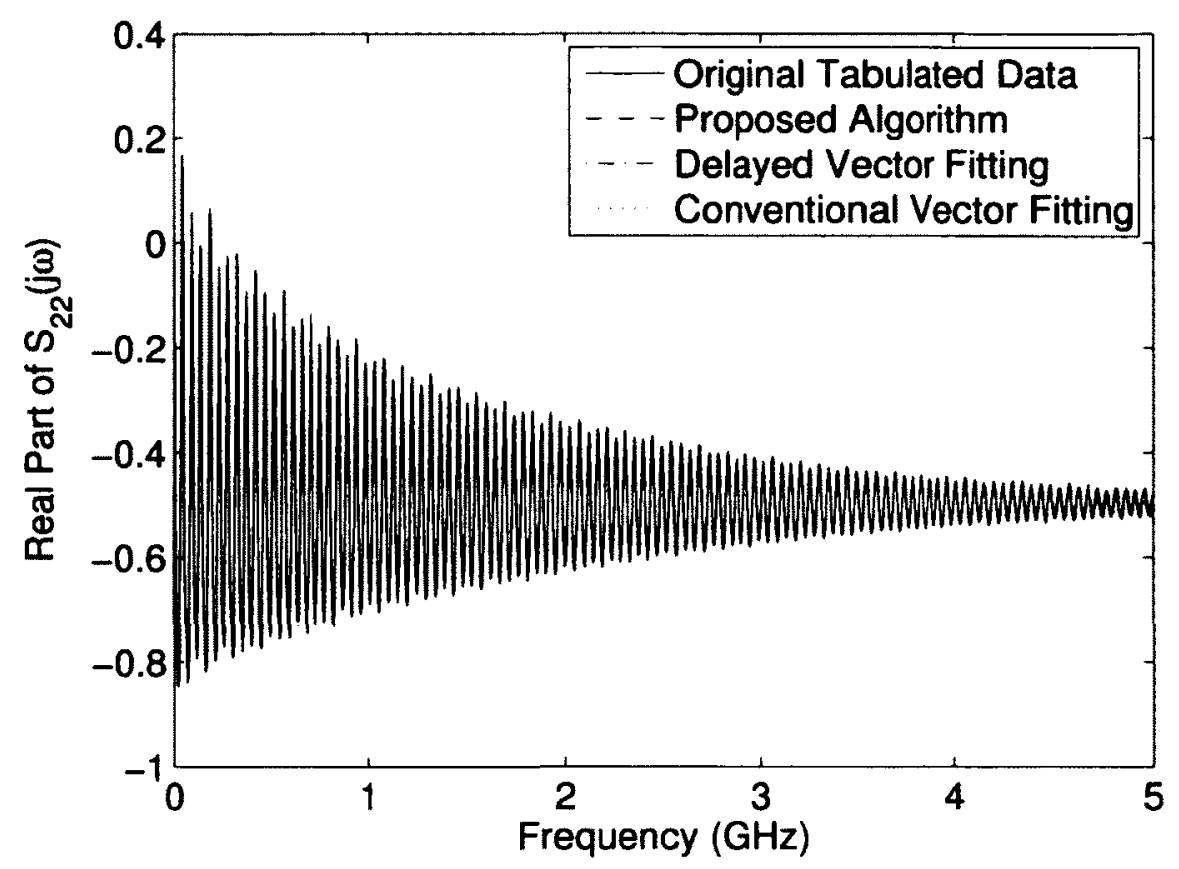

(a) Frequency-domain responses (all the responses overlap).

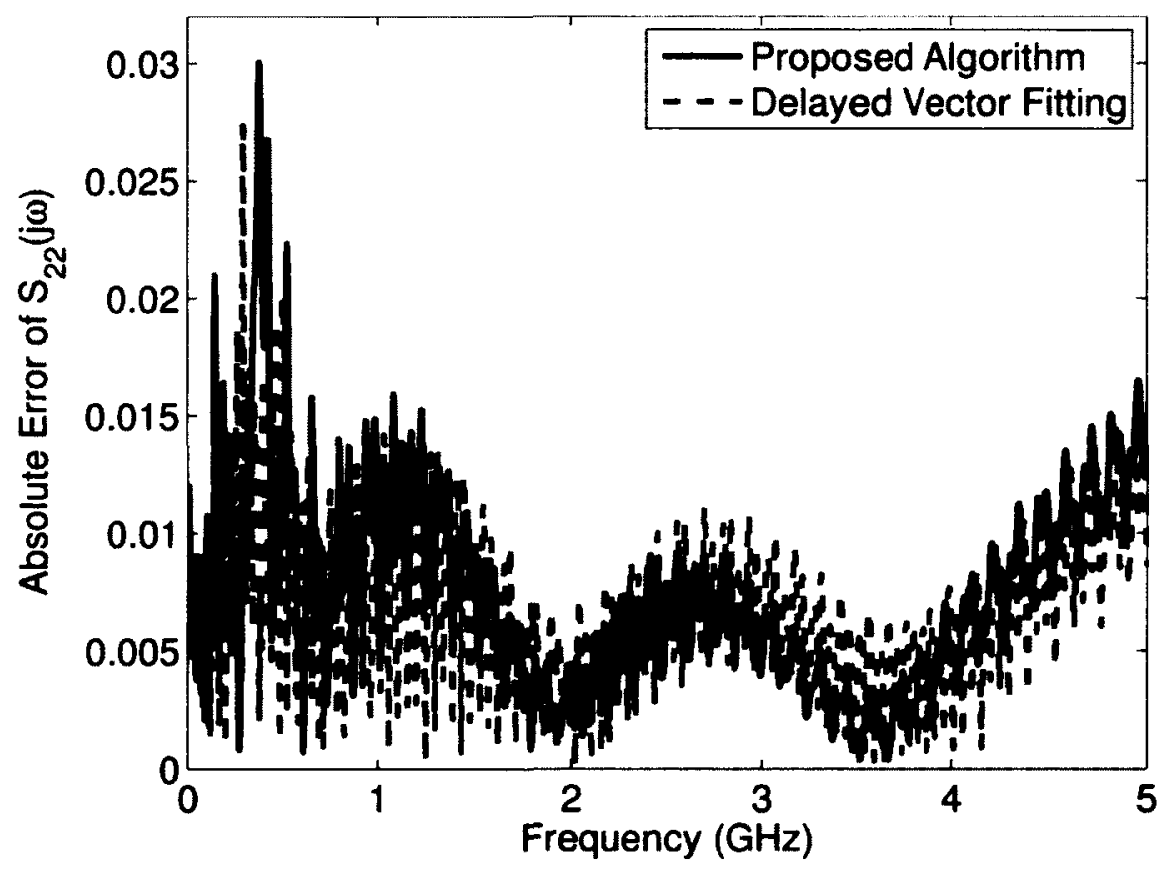

(b) Absolute error.

FIGURE 6.5: A sample comparison of the scattering parameters, $S_{22}(j \omega)$, for example 2 . 
TABLE 6.2: A comparison of macromodel accuracy for example 2 (DRF: Delayed Rational Function; RMSE: Root Mean Squared Error).

\begin{tabular}{|c|c|c|c|}
\hline & Vector Fit & DRF ( $[29])$ & Proposed \\
\hline \hline $\begin{array}{c}S_{11}(s) ; S_{12}(s) ; \\
S_{21}(s) ; S_{22}(s)\end{array}$ & $\begin{array}{c}220 \\
\text { poles } \\
\text { for each } \\
\text { parameter } \\
\text { in the S } \\
\text { matrix }\end{array}$ & $\begin{array}{c}\text { 12 DRF terms } \\
\text { with 9 poles } \\
\text { each for } \\
S_{11}, S_{12}, S_{21} ; \\
16 \text { DRF terms } \\
\text { with 9 poles } \\
\text { each for } S_{22}\end{array}$ & $\begin{array}{c}7 \text { DRF terms } \\
\text { with 9 poles } \\
\text { each for } \\
S_{11}, S_{12}, S_{21} ; \\
9 \text { DRF terms } \\
\text { with 9 poles } \\
\text { each for } S_{22}\end{array}$ \\
\hline $\begin{array}{c}\text { Total Number of } \\
\text { Pole/Residue Terms }\end{array}$ & 880 & 468 & 270 \\
\hline RMSE & 0.0006 & 0.0134 & 0.0057 \\
\hline Transient CPU Time (sec) & 29.97 & 8.30 & 4.09 \\
\hline Eye Diagram Opening (Volts) & 0.961 & 0.974 & 0.979 \\
\hline Eye Diagram CPU Time (sec) & 3195.10 & 1249.64 & 486.82 \\
\hline
\end{tabular}

Next, the accuracy of the proposed algorithm was cross-verified in the time-domain. Each macromodel was connected in the circuit as shown in Fig. 6.6. Subsequently, the circuit was excited using trapezoidal pulse with $0.1 \mathrm{~ns}$ rise/fall times and $20 \mathrm{~ns}$ pulse width. A sample comparison of transient results is given in Fig. 6.7. As seen, the responses of all three macromodels are in agreement. In addition, the CPU times for each of the transient simulations were recorded and are shown in Table 6.2. The proposed macromodel provided a speedup by a factor of 2 and 13 compared to the delayed vector fitting and conventional vector fitting macromodels, respectively.

In order to illustrate the benefits of the proposed macromodel in a real practical setting, eye diagrams for each macromodel were generated. For this purpose, each macromodel was connected to a random bit source with $0.1 \mathrm{~ns}$ rise/fall times and a bit rate of $1 \mathrm{Gbits} /$ second. The analysis is run over 9000 cycles and the output signal over each period ( $2 \mathrm{~ns})$ of the response was superimposed. The eye diagrams for the proposed, delayed vector fitting, and conventional vector fitting macromodels 


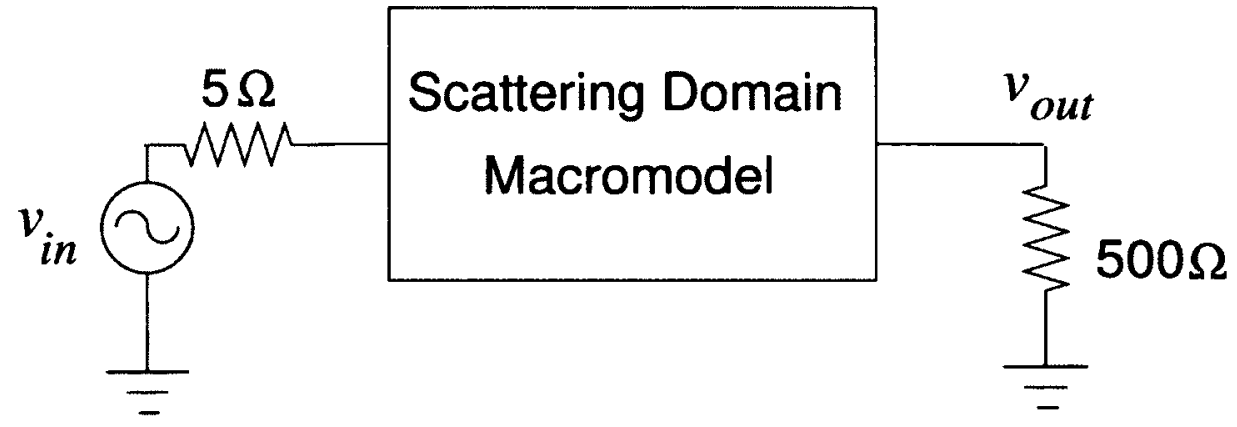

FIGURE 6.6: Circuit for transient analysis of example 2 .

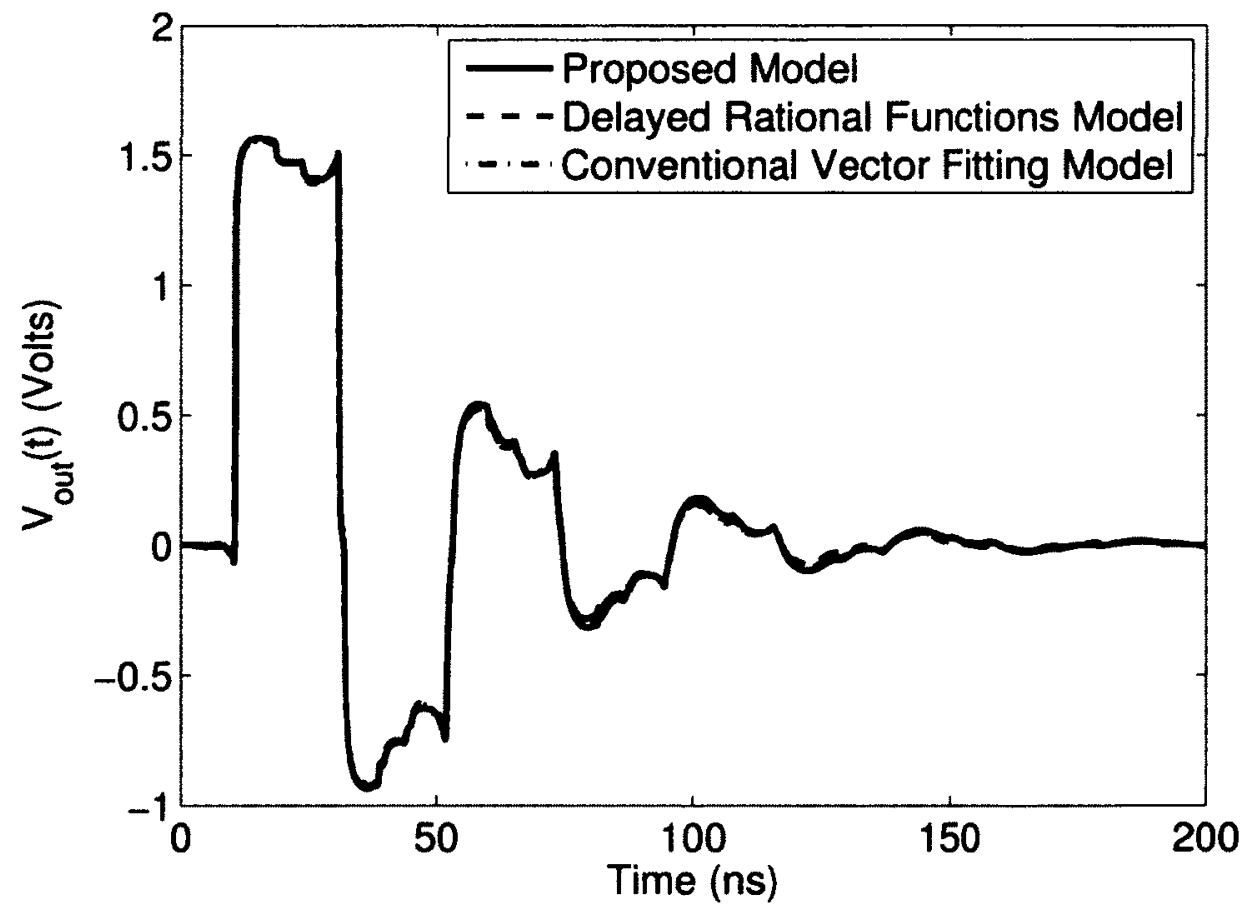

FIGURE 6.7: Comparison of transient responses for example 2 (all the responses overlap). 


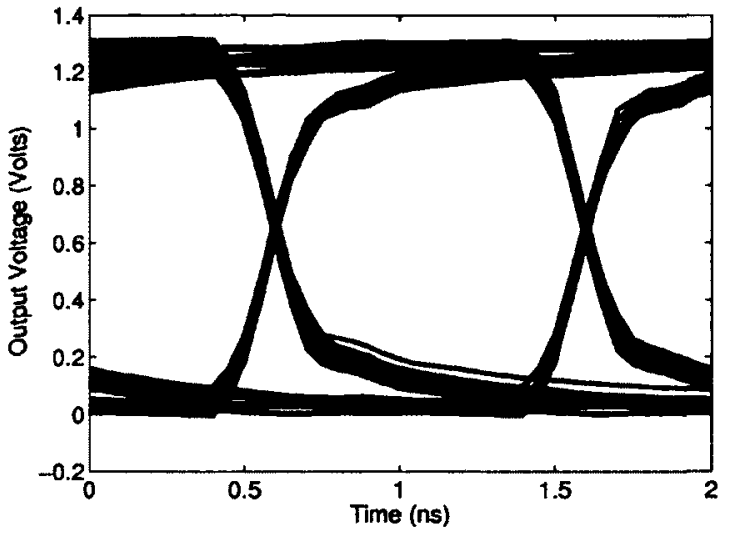

(a) Proposed macromodel.

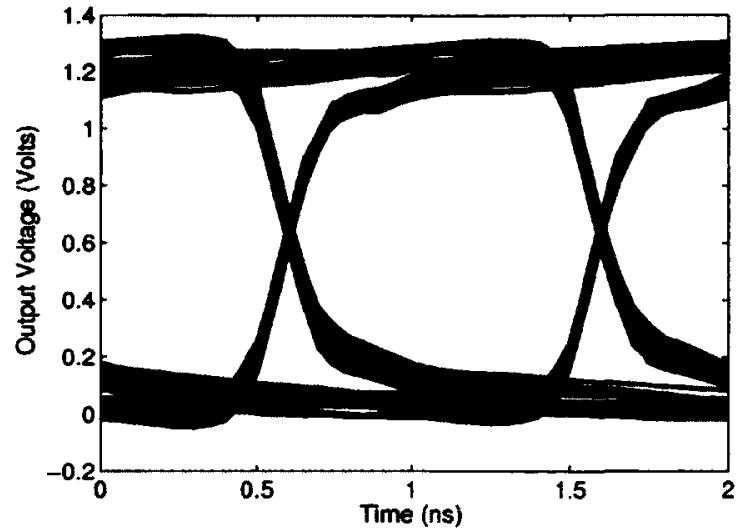

(b) Delayed vector fitting macromodel.

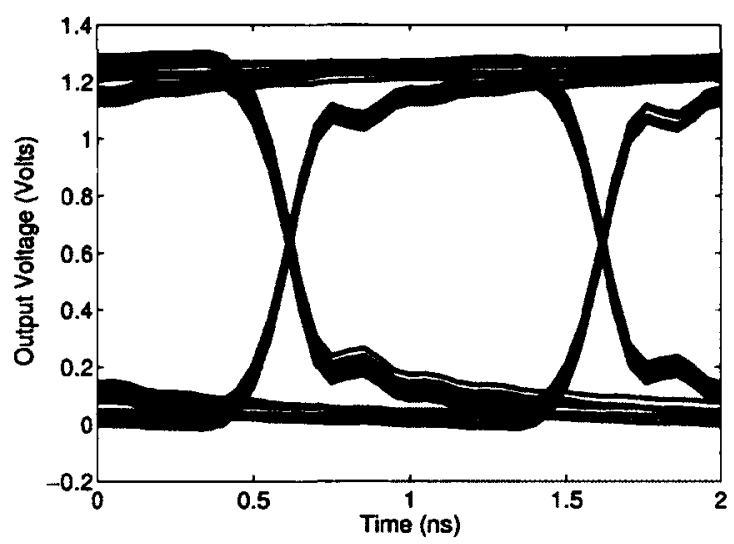

(c) Conventional vector fitting macromodel.

FIGURE 6.8: Eye diagrams for example 2.

are shown in Fig. 6.8. As seen, the eye diagrams from all three macromodels are in agreement. The size of the openings for all three macromodels are nearly identical and the results are summarized in Table 6.2. In addition, a savings of about 760 seconds or 12.5 minutes is achieved using the proposed macromodel over delayed vector fitting. The transient simulations in this example were performed using linear terminations. For the case of nonlinear terminations, further computational savings would be achieved. 


\subsection{Summary}

In this chapter, a general formulation for macromodeling tabulated networks with long periodic delays was presented. The proposed macromodel leads to a reduction in the order of the macromodel compared to conventional delayed rational approximations. Also, an algorithm for stability verification and enforcement was presented. 


\section{CHAPTER 7}

\section{Passive Model-Order Reduction of}

\section{RLC Circuits with Embedded \\ Time-Delay Descriptor (TDD)}

\section{Systems}

In this chapter, a new algorithm for passive model-order reduction of RLC networks with embedded general Time-Delay Descriptor (TDD) systems is presented. In addition, a new passivity verification algorithm for TDD systems is developed. The chapter is organized as follows. Section 7.1 introduces the topic and provides motivation for the new method. Section 7.2 reviews model-order reduction and passivity verification techniques for TDD systems. Next, Section 7.3 develops the new passive model-order reduction algorithm for RLC circuits with embedded TDD systems. Numerical results validating the accuracy and efficiency of the proposed techniques are 
presented in Section 7.4.

The work in this chapter first appeared in [73].

\subsection{Introduction}

Modern high-speed designs contain a massive number of submodules, each of which is characterized as a lumped RLC circuit, distributed interconnect, state-space system, or using a general Time-Delay Descriptor (TDD) system. For the purpose of global transient analysis, all of these subnetworks are combined to yield an enormous system of equations describing the overall network. Prominent methodologies for fast signal integrity analysis of large-order systems are based on model-order reduction [7], [8], [62], [64]-[66]. These techniques generate a reduced-order model that accurately characterizes the input/output port responses of the original large network.

In addition to accuracy, it is important that the reduced-order model retains the physical properties of stability and passivity from the original network. Passive modelorder reduction of RLC circuits has been successfully addressed using Krylov-subspace based techniques (such as PRIMA [8]). For RLC networks that contain embedded high-speed modules described by passive linear state-space and descriptor systems, several passive model-order reduction techniques have been developed [62], [64]. However, these passive reduction techniques cannot directly handle embedded networks described by Time-Delay Descriptor (TDD) systems (such as the DRF macromodels developed in [26]-[34], [54]-[60], [100], [101]).

Recently, several model-order reduction algorithms targeted at large circuit networks with embedded TDD systems have been developed [65], [66]. In [66], Poor Man's Truncated Balanced Realization (PMTBR) [62] was extended to TDD sys- 
tems. In [65], the exponential terms corresponding to time-delays are expanded in the frequency-domain using a Taylor series and subsequently, the moments of the network are obtained via Krylov-subspace techniques. Furthermore, for the specific case of RLC networks embedded with lossless transmission lines with time-delays, such congruence transform-based reduction techniques were shown to guarantee the passivity of the reduced-order network. However, for general TDD networks, neither PMTBR nor Krylov-subspace based techniques guarantee that the resulting reducedorder macromodel is passive. Consequently, passive model-order reduction of RLC networks with embedded TDD systems remains an open problem.

Since the reduced-order macromodels obtained from [65], [66] are not guaranteed to be passive, it is necessary to verify their passivity prior to transient analysis. In the previous chapters, efficient passivity verification techniques were introduced for DRF macromodels. However, these techniques are not directly applicable to general TDD systems. In [102] and [103], a set of sufficient conditions for passivity verification of general time-delay state-space systems were presented in the form of linear matrix inequalities. Recently, in [104] these results were extended to the case of TDD systems with one time-delay. However, for general descriptor systems with multiple time-delays, passivity verification criteria are not available.

In order to address the above issues, this chapter presents a novel passive modelorder reduction algorithm for RLC networks with embedded general TDD systems. In addition, a new set of sufficient passivity conditions for passivity verification of general TDD systems with multiple time-delays is introduced. Numerical results validating the accuracy and efficiency of the proposed algorithms are provided. 


\subsection{Problem Formulation}

A brief review of network formulations for RLC circuits with embedded TDD systems and delay-based model-order reduction is provided in this section. Passivity criteria for TDD systems are also discussed.

\subsubsection{Formulation of Network Equations}

Consider an $n_{\phi}$-port network, $\phi$, of RLC elements described using Modified Nodal Analysis (MNA) as [105]

$$
\begin{aligned}
C_{\phi} \dot{\boldsymbol{x}}_{\phi}(t)+\boldsymbol{G}_{\phi} \boldsymbol{x}_{\phi}(t) & =\boldsymbol{b}_{\phi} \boldsymbol{v}_{p}(t) \\
\boldsymbol{i}_{p}(t) & =\boldsymbol{b}_{\phi}^{T} \boldsymbol{x}_{\phi}(t)
\end{aligned}
$$

where $\boldsymbol{C}_{\phi} \geq \mathbf{0},\left(\boldsymbol{G}_{\phi}+G_{\phi}^{T}\right) \geq \mathbf{0} \in \mathbb{R}^{N_{\phi} \times N_{\phi}}$ are the matrices of memory and memoryless elements, respectively. $\boldsymbol{b}_{\phi} \in \mathbb{R}^{N_{\phi} \times n_{\phi}}$ is a selector matrix mapping the port voltages to the node space of the RLC network. Vectors $v_{p}(t)$ and $i_{p}(t) \in \mathbb{R}^{n_{\phi}}$ define the port voltages and currents, respectively. $\boldsymbol{x}_{\phi}(t) \in \mathbb{R}^{N_{\phi}}$ is the vector of waveforms for the node voltages, inductor currents, and voltage source currents.

Next, consider that the above RLC network $\phi$ is connected to an $n_{\psi}$-port subnetwork, $\psi$, with $K$ time-delays modeled using any of the delay-based techniques in [26]-[34], [54]-[60], [100], [101]. An illustration of the overall network is given in Fig. 7.1. The general form for any of these embedded time-delay systems is

$$
\begin{aligned}
\boldsymbol{E} \dot{\boldsymbol{x}}_{\psi}(t) & =\boldsymbol{A} \boldsymbol{x}_{\psi}(t)+\sum_{k=1}^{K} \boldsymbol{A}_{k} \boldsymbol{x}_{\psi}\left(t-\tau_{k}\right)+\boldsymbol{B} \boldsymbol{v}_{\psi}(t) \\
\boldsymbol{i}_{\psi}(t) & =\boldsymbol{C} \boldsymbol{x}_{\psi}(t)+\boldsymbol{D} \boldsymbol{v}_{\psi}(t)
\end{aligned}
$$


where $E \in \mathbb{R}^{N_{\psi} \times N_{\psi}}$ could be either a singular or nonsingular matrix, $A, A_{k} \in$ $\mathbb{R}^{N_{\psi} \times N_{\psi}}, \boldsymbol{B}, C^{T} \in \mathbb{R}^{N_{\psi} \times n_{\psi}}, D \in \mathbb{R}^{n_{\psi} \times n_{\psi}}, x_{\psi}(t) \in \mathbb{R}^{N_{\psi}}$. Vectors $i_{\psi}(t), \boldsymbol{v}_{\psi}(t) \in \mathbb{R}^{n_{\psi}}$ represent the output currents and input voltages, respectively. Time-delay systems in the form of (7.2) are referred to as regular systems if the matrix $\boldsymbol{E}$ is nonsingular (or state-space if $\boldsymbol{E}$ is the identity matrix) and singular or descriptor systems if $\boldsymbol{E}$ is singular [106]. The work in this chapter is developed for Time-Delay Descriptor (TDD) systems, however, it is also applicable to time-delay state-space systems. Embedding this subnetwork into the MNA equations in (7.1) yields the descriptor system

$$
\begin{aligned}
\boldsymbol{H} \dot{\boldsymbol{x}}(t)+\boldsymbol{G} \boldsymbol{x}(t)+\sum_{k=1}^{K} \boldsymbol{G}_{k} \boldsymbol{x}\left(t-\tau_{k}\right) & =\boldsymbol{b}_{p}(t) \\
\boldsymbol{i}_{p}(t) & =\boldsymbol{b}^{T} \boldsymbol{x}(t)
\end{aligned}
$$

where

$$
\begin{gathered}
\boldsymbol{x}(t)=\left[\begin{array}{c}
\boldsymbol{v}_{\phi}(t) \\
\hline \boldsymbol{x}_{\psi}(t) \\
\boldsymbol{i}_{\psi}(t)
\end{array}\right], \quad \boldsymbol{H}=\left[\begin{array}{c|cc}
\boldsymbol{C}_{\phi} & \mathbf{0} & \mathbf{0} \\
\hline \mathbf{0} & \boldsymbol{E} & \mathbf{0} \\
\mathbf{0} & \mathbf{0} & \mathbf{0}
\end{array}\right], \quad \boldsymbol{G}=\left[\begin{array}{c|cc}
\boldsymbol{G}_{\phi} & \mathbf{0} & \boldsymbol{L} \\
\hline \mathbf{0} & -\boldsymbol{A} & -\boldsymbol{B} \\
-\boldsymbol{L}^{T} & \boldsymbol{C} & \boldsymbol{D}
\end{array}\right], \\
\boldsymbol{G}_{k}=\left[\begin{array}{c|cc}
\mathbf{0} & \mathbf{0} & \mathbf{0} \\
\hline \mathbf{0} & -\boldsymbol{A}_{k} & \mathbf{0} \\
\mathbf{0} & \mathbf{0} & \mathbf{0}
\end{array}\right], \quad \boldsymbol{b}=\left[\begin{array}{c}
\boldsymbol{b}_{\phi} \\
\hline \mathbf{0} \\
\mathbf{0}
\end{array}\right] .
\end{gathered}
$$

$\boldsymbol{L} \in \mathbb{R}^{N_{\phi} \times n_{\psi}}$ is a selector matrix that maps the port output current of $\boldsymbol{i}_{\psi}(t)$ into the node space of the RLC network $\phi$. The port voltages of the embedded module are also related to the node voltages by $\boldsymbol{v}_{\psi}(t)=\boldsymbol{L}^{T} \boldsymbol{v}_{\phi}(t)$. Following the above process, it is clear that any number of embedded modules can be accommodated. 


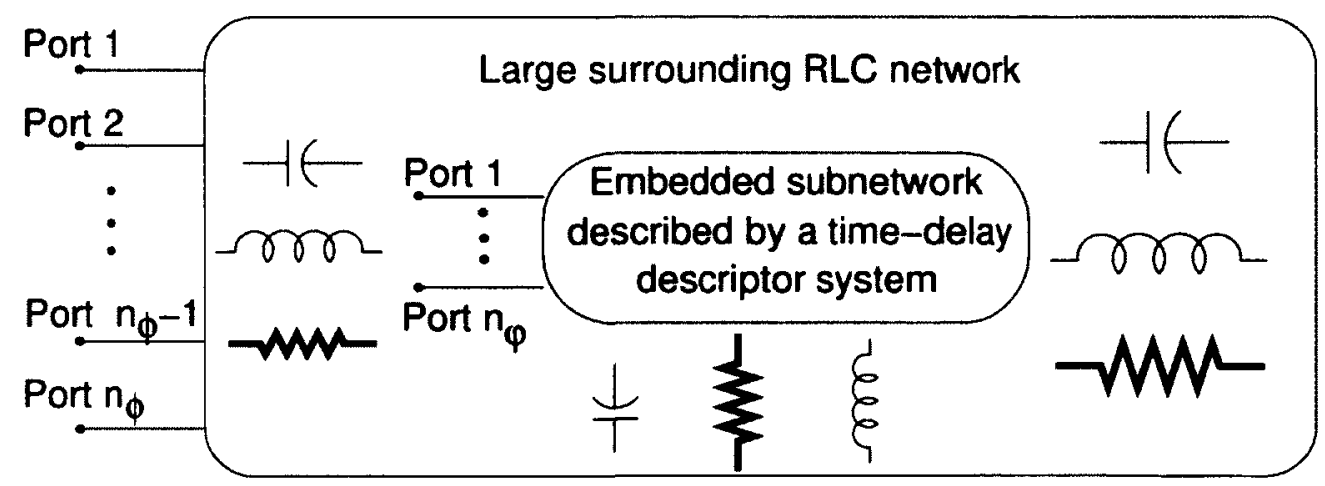

FIGURE 7.1: RLC circuit with an embedded subnetwork described by a time-delay descriptor system.

\subsubsection{Model-Order Reduction of Time-Delay Systems}

Several algorithms can be found in the literature for model-order reduction of RLC networks with embedded TDD systems [65], [66]. In each technique, the first step is to obtain left and right orthonormal projection matrices $\boldsymbol{U}, \boldsymbol{V} \in \mathbb{R}^{N \times q}$ (for congruence transform-based reduction algorithms $U=V$ ), where $N$ and $q$ are the sizes of the original system in (7.3) and reduced system in (7.5), respectively. Next, the reducedorder system is found by mapping the vector $\boldsymbol{x}(t) \in \mathbb{R}^{N}$ to a smaller vector $\hat{\boldsymbol{x}}(t) \in \mathbb{R}^{q}$. Equation (7.3a) is also multiplied by $\boldsymbol{U}^{T}$ to give the reduced system

$$
\begin{aligned}
\hat{\boldsymbol{H}} \dot{\hat{\boldsymbol{x}}}(t)+\hat{\boldsymbol{G}} \hat{\boldsymbol{x}}(t)+\sum_{k=1}^{K} \hat{\boldsymbol{G}}_{k} \hat{\boldsymbol{x}}\left(t-\tau_{k}\right) & =\hat{\boldsymbol{b}} \boldsymbol{v}_{p}(t) \\
i_{p}(t) & =\hat{\boldsymbol{b}}^{T} \hat{\boldsymbol{x}}(t)
\end{aligned}
$$

where $\boldsymbol{x}(t)=\boldsymbol{V} \hat{\boldsymbol{x}}(t)$ and

$$
\hat{\boldsymbol{H}}=\boldsymbol{U}^{T} \boldsymbol{H} \boldsymbol{V}, \quad \hat{\boldsymbol{G}}=\boldsymbol{U}^{T} \boldsymbol{G} \boldsymbol{V}, \quad \hat{\boldsymbol{G}}_{k}=\boldsymbol{U}^{T} \boldsymbol{G}_{k} \boldsymbol{V}, \quad \hat{\boldsymbol{b}}=\boldsymbol{U}^{T} \boldsymbol{b}
$$


For the specific case of RLC networks with embedded TDD systems derived from lossless transmission lines, [65] presents an algorithm for passive model-order reduction of such a system. However, for RLC networks with embedded subnetworks described by general TDD systems in the form of (7.2), the passivity of the reducedorder model is not guaranteed.

\subsubsection{Passivity Criteria for Time-Delay Systems}

For a system in the form of (7.2), the following definition for passivity in the timedomain is adopted [87], [102], [104].

Definition 7.1: The network in (7.2) is passive if

$$
\int_{0}^{t} v^{T}(\eta) i(\eta) d \eta \geq 0
$$

for all $t \geq 0$ and zero initial conditions. Here, $\boldsymbol{v}(t)=\boldsymbol{v}_{\psi}(t)$ and $\boldsymbol{i}(t)=\boldsymbol{i}_{\psi}(t)$ are the voltages and currents, respectively, of the network in (7.2).

Equivalently, in the Laplace-domain it is necessary that the transfer function of (7.2) given by

$$
\boldsymbol{Y}(s)=\boldsymbol{C}\left(s \boldsymbol{E}-\boldsymbol{A}-\sum_{k=1}^{K} \boldsymbol{A}_{k} e^{-s \tau_{k}}\right)^{-1} \boldsymbol{B}+\boldsymbol{D}
$$

satisfies the following 3 conditions [86]:

a) $\boldsymbol{Y}(s)$ is analytic for all values of $s$ with $\mathfrak{R e}(s)>0$;

b) $\boldsymbol{Y}\left(s^{*}\right)=\boldsymbol{Y}^{*}(s)$, where the superscript ' $*$ ' is the complex conjugate operator; 
c) $\left[\boldsymbol{Y}^{H}(s)+\boldsymbol{Y}(s)\right]$ is nonnegative definite for $\mathfrak{R e}(s)>0$. That is

$$
\boldsymbol{z}^{H}\left[\boldsymbol{Y}^{H}(s)+\boldsymbol{Y}(s)\right] \boldsymbol{z} \geq 0
$$

for all complex values of $s$ with $\mathfrak{R e}(s)>0$ and for any arbitrary vector $z$. The superscript ' $H$ ' represents the Hermitian conjugate operator.

Direct verification of these criteria is computationally infeasible since it requires a frequency-sweep of the entire right-half plane.

In [71], for the case of delayed rational function based macromodels, the above issue was addressed wherein the passivity verification region described by condition c) was reduced from the entire right-half plane to just the imaginary axis. However, in this technique, an assumption was made that the rational functions corresponding to nonzero delays are all strict rational. Consequently, for general time-delay systems in the form of (7.2) (which may not be in strict rational form), these techniques cannot be applied.

In [102] and [103], a set of sufficient conditions for passivity verification of general time-delay state-space systems were presented in the form of linear matrix inequalities. Recently, in [104] these results were extended to the case of TDD systems with one time-delay. However, for general descriptor systems with multiple time-delays, passivity verification criteria is still an open problem.

\subsection{Proposed Passive Reduction Algorithm}

In this section, a passive model-order reduction algorithm is developed for RLC circuits with embedded subnetworks described by general TDD systems. The new algo- 
rithm is based on extending the Passive Reduction algorithm for RLC interconnect circuits with Embedded State-space Systems (PRESS) [64] to handle embedded TDD systems. Also, the passivity verification technique from [104] is extended to handle TDD systems with multiple delays.

\subsubsection{Passivity Verification of TDD Systems}

As a first step in the proposed reduction algorithm, it is essential to verify the passivity of the embedded TDD system. The following theorem introduces sufficient passivity criteria based on linear matrix inequalities.

Theorem 7.1: The TDD system in the form of (7.2) is passive if there exists a matrix $\boldsymbol{P}$ and symmetric positive semi-definite matrices $\boldsymbol{P}_{1} \geq 0, \boldsymbol{P}_{2} \geq 0, \ldots, \boldsymbol{P}_{K} \geq 0$, such that the following linear matrix inequalities hold:

$$
\boldsymbol{\Omega}=\left[\begin{array}{cccccc}
\boldsymbol{A}^{T} \boldsymbol{P}^{T}+\boldsymbol{P} \boldsymbol{A}+\sum_{k=1}^{K} \boldsymbol{P}_{k} & \boldsymbol{P A}_{1} & \cdots & \boldsymbol{P} \boldsymbol{A}_{K} & -\left(\boldsymbol{C}^{T}-\boldsymbol{P B}\right) \\
\boldsymbol{A}_{1}^{T} \boldsymbol{P}^{T} & -\boldsymbol{P}_{1} & \cdots & \mathbf{0} & \mathbf{0} \\
\vdots & \vdots & \ddots & \vdots & \vdots \\
\boldsymbol{A}_{K}^{T} \boldsymbol{P}^{T} & \mathbf{0} & \cdots & -\boldsymbol{P}_{K} & 0 \\
-\left(\boldsymbol{C}-\boldsymbol{B}^{T} \boldsymbol{P}^{T}\right) & \mathbf{0} & \cdots & \mathbf{0} & -\left(\boldsymbol{D}+\boldsymbol{D}^{T}\right)
\end{array}\right] \leq \mathbf{0}
$$

Proof of Theorem 7.1: Define the positive semi-definite Lyapunov-Krasovskii function

$$
V\left(\boldsymbol{x}_{t}\right)=\boldsymbol{x}^{T}(t) \boldsymbol{E} \boldsymbol{P} \boldsymbol{x}(t)+\sum_{k=1}^{K} \int_{t-\tau_{k}}^{t} \boldsymbol{x}(s)^{T} \boldsymbol{P}_{k} \boldsymbol{x}(s) d s \geq 0
$$


where

$$
\boldsymbol{x}_{t}=\boldsymbol{x}(t+\beta), \quad \beta \in\left(-\tau_{\max }, 0\right]
$$

and $\tau_{\max }=\max \left\{0, \tau_{1}, \ldots, \tau_{K}\right\}$. Taking the time derivative of $(7.11)$ along the solution of (7.2) gives

$$
\dot{V}\left(\boldsymbol{x}_{t}\right)=\dot{\boldsymbol{x}}^{T}(t) \boldsymbol{E}^{T} \boldsymbol{P}^{T} \boldsymbol{x}(t)+\boldsymbol{x}^{T}(t) \boldsymbol{P} \boldsymbol{E} \dot{\boldsymbol{x}}(t)+\sum_{k=1}^{K}\left[\boldsymbol{x}^{T}(t) \boldsymbol{P}_{k} \boldsymbol{x}(t)-\boldsymbol{x}^{T}\left(t-\tau_{k}\right) \boldsymbol{P}_{k} \boldsymbol{x}\left(t-\tau_{k}\right)\right]
$$

Then using $\boldsymbol{E} \dot{\boldsymbol{x}}(t)$ from (7.2a), with $\boldsymbol{x}(t)=\boldsymbol{x}_{\psi}(t)$ and input $\boldsymbol{v}(t)=\boldsymbol{v}_{\psi}(t)$, yields

$$
\begin{aligned}
\dot{V}\left(\boldsymbol{x}_{t}\right)= & \boldsymbol{x}^{T}(t)\left[\boldsymbol{A}^{T} \boldsymbol{P}^{T}+\boldsymbol{P} \boldsymbol{A}\right] \boldsymbol{x}(t)+\boldsymbol{v}^{T}(t) \boldsymbol{B}^{T} \boldsymbol{P}^{T} \boldsymbol{x}(t)+\boldsymbol{x}^{T}(t) \boldsymbol{P} \boldsymbol{B} \boldsymbol{v}(t) \\
& +\sum_{k=1}^{K}\left[\boldsymbol{x}^{T}\left(t-\tau_{k}\right) \boldsymbol{A}_{k}^{T} \boldsymbol{P}^{T} \boldsymbol{x}(t)+\boldsymbol{x}^{T}(t) \boldsymbol{P} \boldsymbol{A}_{k} \boldsymbol{x}\left(t-\tau_{k}\right)\right] \\
& +\sum_{k=1}^{K}\left[\boldsymbol{x}^{T}(t) \boldsymbol{P}_{k} \boldsymbol{x}(t)-\boldsymbol{x}^{T}\left(t-\tau_{k}\right) \boldsymbol{P}_{k} \boldsymbol{x}\left(t-\tau_{k}\right)\right] .
\end{aligned}
$$

Define the Hamiltonian

$$
H\left(x_{t}, t\right)=-\dot{V}\left(x_{t}\right)+2 i^{T}(t) v(t)
$$

where $i(t)=i_{\psi}(t)$ is the output of the system in (7.2b). Substituting $i(t)$ from (7.2b) and $V\left(\boldsymbol{x}_{t}\right)$ from $(7.14)$ in $(7.15)$ gives

$$
\begin{aligned}
H\left(\boldsymbol{x}_{t}, t\right)= & -\boldsymbol{x}^{T}(t)\left[\boldsymbol{A}^{T} \boldsymbol{P}^{T}+\boldsymbol{P} \boldsymbol{A}\right] \boldsymbol{x}(t)-\sum_{k=1}^{K}\left[\boldsymbol{x}^{T}(t) \boldsymbol{P}_{k} \boldsymbol{x}(t)-\boldsymbol{x}^{T}\left(t-\tau_{k}\right) \boldsymbol{P}_{k} \boldsymbol{x}\left(t-\tau_{k}\right)\right] \\
& -\sum_{k=1}^{K}\left[\boldsymbol{x}^{T}\left(t-\tau_{k}\right) \boldsymbol{A}_{k}^{T} \boldsymbol{P}^{T} \boldsymbol{x}(t)+\boldsymbol{x}^{T}(t) \boldsymbol{P} \boldsymbol{A}_{k} \boldsymbol{x}\left(t-\tau_{k}\right)\right]+\boldsymbol{v}^{T}(t)\left[\boldsymbol{D}+\boldsymbol{D}^{T}\right] \boldsymbol{v}(t) \\
& -\boldsymbol{v}^{T}(t)\left[\boldsymbol{B}^{T} \boldsymbol{P}^{T}-\boldsymbol{C}\right] \boldsymbol{x}(t)-\boldsymbol{x}^{T}(t)\left[\boldsymbol{P B}-\boldsymbol{C}^{T}\right] \boldsymbol{v}(t)
\end{aligned}
$$


This can be written as

$$
H\left(x_{t}, t\right)=-\left[\begin{array}{lllll}
x^{T}(t) & x^{T}\left(t-\tau_{1}\right) & \cdots & x^{T}\left(t-\tau_{K}\right) & \boldsymbol{v}^{T}(t)
\end{array}\right] \boldsymbol{\Omega}\left[\begin{array}{c}
x(t) \\
x\left(t-\tau_{1}\right) \\
\vdots \\
x\left(t-\tau_{K}\right) \\
v(t)
\end{array}\right]
$$

where $\boldsymbol{\Omega}$ is defined as (7.10b). From (7.10b), $\boldsymbol{\Omega} \leq \mathbf{0}$ and the Hamiltonian in (7.17) satisfies

$$
H\left(x_{t}, t\right)=-\dot{V}\left(x_{t}\right)+2 i^{T}(t) v(t) \geq 0
$$

Rearranging the equation and integrating both sides gives

$$
\int_{t_{0}}^{t_{1}}\left[\boldsymbol{i}^{T}(t) v(t)\right] d t \geq 1 / 2\left[V\left(x_{t_{1}}\right)-V\left(x_{t_{0}}\right)\right]
$$

For passivity in the immitance domain, recall from Definition 7.1 that the system is passive if the integral on the left is positive semi-definite for $t_{0}=0$. Since $V\left(\boldsymbol{x}_{t}\right) \geq 0$ for $\boldsymbol{x}_{t} \neq 0, V\left(\boldsymbol{x}_{t}\right)=0$ for $\boldsymbol{x}_{t}=\mathbf{0}$, and $\boldsymbol{x}_{\left(t_{0}=0\right)}=\mathbf{0}$ (for zero initial conditions), this gives

$$
\int_{0}^{t_{1}}\left[\boldsymbol{i}^{T}(t) v(t)\right] d t \geq 1 / 2\left[V\left(x_{t_{1}}\right)\right] \geq 0 .
$$

Comparing (7.20) and Definition 7.1, is clear that (7.2) is passive.

Using Theorem 7.1 , if a matrix $\boldsymbol{P}$ and positive semi-definite matrices $\boldsymbol{P}_{1}, \boldsymbol{P}_{2}, \ldots$, $\boldsymbol{P}_{K}$ can be found that satisfy (7.10), then the TDD subnetwork is passive. 


\subsubsection{Formulation of Unified Network Equations}

In this section, a unified formulation involving RLC networks with embedded TDD systems is given. The new formulation guarantees passive reduced-order models are obtained using congruence transform-based model-order reduction techniques. A proof of this fact is provided below in Section 7.3.4.

First, (7.2a) is multiplied by the transformation matrix $\boldsymbol{P} \in \mathbb{R}^{N_{\psi} \times N_{\psi}}$, obtained from solving (7.10). This yields

$$
\boldsymbol{P E} \dot{\boldsymbol{x}}_{\psi}(t)=\boldsymbol{P} \boldsymbol{A} \boldsymbol{x}_{\psi}(t)+\sum_{k=1}^{K} \boldsymbol{P} \boldsymbol{A}_{k} \boldsymbol{x}_{\psi}\left(t-\tau_{k}\right)+\boldsymbol{P} \boldsymbol{B} \boldsymbol{i}_{\psi}(t)
$$

Next, embedding the transformed network described by (7.21) and (7.2b) in the MNA formulation of the RLC network $\phi$ in (7.1) gives

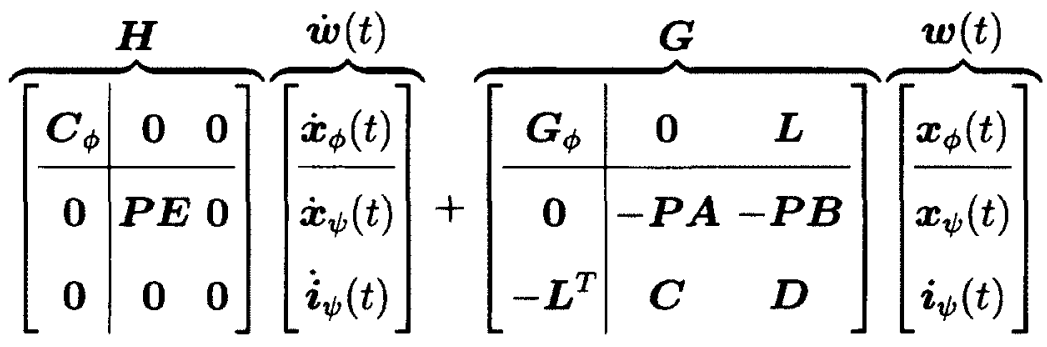

$$
\begin{aligned}
& +\sum_{k=1}^{K} \overbrace{\left[\begin{array}{r|rr}
\mathbf{0} & \mathbf{0} & \mathbf{0} \\
\mathbf{0} & -\boldsymbol{P} \boldsymbol{A}_{k} \mathbf{0} \\
\mathbf{0} & \mathbf{0} & \mathbf{0}
\end{array}\right]}^{\boldsymbol{G}_{k}} \overbrace{\left[\begin{array}{l}
\boldsymbol{x}_{\phi}\left(t-\tau_{k}\right) \\
\boldsymbol{x}_{\psi}\left(t-\tau_{k}\right) \\
\boldsymbol{i}_{\psi}\left(t-\tau_{k}\right)
\end{array}\right]}^{\boldsymbol{w}\left(t-\tau_{k}\right)}=\overbrace{\left[\begin{array}{c}
\boldsymbol{b}_{\phi} \\
\mathbf{0} \\
\mathbf{0}
\end{array}\right]}^{\boldsymbol{b}} \boldsymbol{v}_{p}(t) \\
& i_{p}(t)=\boldsymbol{b}^{T} \boldsymbol{w}(t) \text {. }
\end{aligned}
$$

In the resulting system (7.22), $\boldsymbol{H}, \boldsymbol{G}, \boldsymbol{G}_{k} \in \mathbb{R}^{N \times N}, \boldsymbol{w}(t) \in \mathbb{R}^{N}$ is the state-vector, $\boldsymbol{b} \in \mathbb{R}^{N \times n_{\phi}}$, and $N=\left(N_{\psi}+N_{\phi}+n_{\psi}\right)$ is the size of the overall network. 
Although pre-multiplying by a matrix $\boldsymbol{P}$ will lead to a dense formulation for the embedded subnetwork (while the MNA formulation for the original RLC network is sparse), it does not have a significant impact on the overall reduction time. This is because the embedded networks are typically quite small (on the order of a few hundred) relative to the sparse RLC network (on the order of several thousand). In addition, model-order reduction results in dense reduced-order models regardless of the sparsity of the original network.

\subsubsection{Passive Model-Order Reduction}

In the proposed passive model-order reduction procedure, the original network in (7.22) is reduced using a congruence transform to give

$$
\begin{aligned}
\hat{\boldsymbol{H}} \dot{\boldsymbol{w}}(t)+\hat{\boldsymbol{G}} \hat{\boldsymbol{w}}(t)+\sum_{k=1}^{K} \hat{\boldsymbol{G}}_{k} \hat{\boldsymbol{w}}\left(t-\tau_{k}\right) & =\hat{\boldsymbol{b}} \boldsymbol{v}_{p}(t) \\
\boldsymbol{i}_{p}(t) & =\hat{\boldsymbol{b}}^{T} \hat{\boldsymbol{w}}(t)
\end{aligned}
$$

where $\boldsymbol{w}(t)=\boldsymbol{Q} \hat{\boldsymbol{w}}(t)$ relates the original to the reduced states and

$$
\hat{\boldsymbol{H}}=\boldsymbol{Q}^{T} \boldsymbol{H} \boldsymbol{Q}, \quad \hat{\boldsymbol{G}}=\boldsymbol{Q}^{T} \boldsymbol{G} \boldsymbol{Q}, \quad \hat{\boldsymbol{G}}_{k}=\boldsymbol{Q}^{T} \boldsymbol{G}_{k} \boldsymbol{Q}, \quad \hat{\boldsymbol{b}}=\boldsymbol{Q}^{T} \boldsymbol{b}
$$

The reduction matrix $Q \in \mathbb{R}^{N \times q}$ is an orthonormal basis obtained applying the Krylov-subspace techniques in [65] on the system in (7.22). The size of the reducedorder model is $q<<N$. A proof that the reduced model represented by (7.23) is passive is given in the following section. 


\subsubsection{Proof of Preservation of Passivity}

First, note that (7.23) is a descriptor system in the form of (7.2) with $\boldsymbol{E}=\hat{\boldsymbol{H}}$, $\boldsymbol{A}=-\hat{\boldsymbol{G}}, \boldsymbol{A}_{k}=-\hat{\boldsymbol{G}}_{k}, \boldsymbol{B}=\hat{\boldsymbol{b}}, \boldsymbol{C}=\hat{\boldsymbol{b}}^{T}$, and $\boldsymbol{D}=\mathbf{0}$. It is desired to prove that the reduced system in (7.23) satisfies linear matrix inequalities in the form of (7.10a) and (7.10b). Equivalently, it is desired to show that there exists a matrix $\hat{\boldsymbol{P}}$ and positive semi-definite matrices $\hat{\boldsymbol{P}}_{1} \geq \mathbf{0}, \hat{\boldsymbol{P}}_{2} \geq \mathbf{0}, \ldots, \hat{\boldsymbol{P}}_{K} \geq \mathbf{0}$ such that the following linear matrix inequalities are satisfied:

$$
\left[\begin{array}{cccccc}
-\hat{\boldsymbol{G}}^{T} \hat{\boldsymbol{P}}^{T}-\hat{\boldsymbol{P}} \hat{\boldsymbol{G}}+\sum_{k=1}^{K} \hat{\boldsymbol{P}}_{k} & -\hat{\boldsymbol{P}} \hat{\boldsymbol{G}}_{1} & \cdots & -\hat{\boldsymbol{P}} \hat{G}_{K} & -(\hat{\boldsymbol{b}}-\hat{\boldsymbol{P}} \hat{\boldsymbol{b}}) \\
-\hat{\boldsymbol{G}}_{1}^{T} \hat{\boldsymbol{P}}^{T} & -\hat{\boldsymbol{P}}_{1} & \cdots & \mathbf{0} & \mathbf{0} \\
\vdots & \vdots & \ddots & \vdots & \vdots \\
-\hat{\boldsymbol{G}}_{K}^{T} \hat{\boldsymbol{P}}^{T} & \mathbf{0} & \cdots & -\hat{\boldsymbol{P}}_{K} & \mathbf{0} \\
-\left(\hat{\boldsymbol{b}}^{T}-\hat{\boldsymbol{b}}^{T} \hat{\boldsymbol{P}}^{T}\right) & \mathbf{0} & \cdots & \mathbf{0} & \mathbf{0}
\end{array}\right] \leq \mathbf{0} .
$$

To achieve this, it will be shown that for $\hat{\boldsymbol{P}}=\boldsymbol{I}_{q}$, where $\boldsymbol{I}_{q}$ is the $q \times q$ identity matrix, there exists $\left\{\hat{\boldsymbol{P}}_{k} \geq \mathbf{0}\right\}$ such that each of the above conditions hold.

To begin, consider condition (7.25a). Expanding $\hat{\boldsymbol{H}}$ and using $\hat{\boldsymbol{P}}=\boldsymbol{I}_{q}$ gives

$$
\hat{\boldsymbol{P}} \hat{\boldsymbol{H}}=\hat{\boldsymbol{H}}=\boldsymbol{Q}^{T} \boldsymbol{H} \boldsymbol{Q}=\boldsymbol{Q}^{T}\left[\begin{array}{ccc}
C_{\phi} & 0 & 0 \\
0 & P E & 0 \\
0 & 0 & 0
\end{array}\right] Q
$$

From (7.10a), $\boldsymbol{P E}=\boldsymbol{E}^{T} \boldsymbol{P}^{T} \geq 0$. In addition, using the MNA formulation for RLC circuits $\boldsymbol{C}_{\phi}=\boldsymbol{C}_{\phi}^{T} \geq \mathbf{0}[8]$. Therefore, it is clear that $\hat{\boldsymbol{H}}=\hat{\boldsymbol{H}}^{T} \geq \mathbf{0}$ and the condition 
in (7.25a) holds.

Next, it is desired to prove that the reduced system in (7.23) satisfies the second condition described in $(7.25 \mathrm{~b})$. Let

$$
\hat{\boldsymbol{P}}_{k}=Q^{T} \overline{\boldsymbol{P}}_{k} \boldsymbol{Q}=\overbrace{\left[\begin{array}{ccc}
T & 0 & 0 \\
0 & \boldsymbol{P}_{k} & 0 \\
0 & 0 & 0
\end{array}\right]}^{\bar{P}_{k}} Q \geq 0
$$

where $\boldsymbol{P}_{1} \geq \mathbf{0}, \boldsymbol{P}_{2} \geq \mathbf{0}, \ldots, \boldsymbol{P}_{K} \geq \mathbf{0}$ are matrices that satisfy (7.10b) for the embedded system. Then using $\hat{\boldsymbol{P}}=\boldsymbol{I}_{q}$, the matrix in (7.25b) can be written as

$$
\mathcal{Q}^{T}\left[\begin{array}{ccccc}
-\boldsymbol{G}^{T}-\boldsymbol{G}+\sum_{k=1}^{K} \overline{\boldsymbol{P}}_{k} & -\boldsymbol{G}_{1} & \cdots & -\boldsymbol{G}_{K} & -(\boldsymbol{b}-\boldsymbol{b}) \\
-\boldsymbol{G}_{1}^{T} & -\overline{\boldsymbol{P}}_{1} & \cdots & \mathbf{0} & \mathbf{0} \\
\vdots & \vdots & \ddots & \vdots & \vdots \\
-\boldsymbol{G}_{K}^{T} & \mathbf{0} & \cdots & -\overline{\boldsymbol{P}}_{K} & \mathbf{0} \\
-\left(\boldsymbol{b}^{T}-\boldsymbol{b}^{T}\right) & \mathbf{0} & \cdots & \mathbf{0} & \mathbf{0}
\end{array}\right] \mathcal{Q}
$$

with

$$
\mathcal{Q}=\left[\begin{array}{cccc}
\boldsymbol{Q} & \mathbf{0} & \cdots & \mathbf{0} \\
\mathbf{0} & \ddots & \ddots & \vdots \\
\vdots & \ddots & \boldsymbol{Q} & \mathbf{0} \\
\mathbf{0} & \cdots & \mathbf{0} & \boldsymbol{I}_{n_{\phi}}
\end{array}\right]
$$

Here, $\mathcal{Q}$ is a diagonal matrix of $(K+1)$ vectors $\boldsymbol{Q}$ and an $n_{\phi} \times n_{\phi}$ identity matrix, $\boldsymbol{I}_{n_{\phi}}$. Recall that a congruence transform does not effect the signature of the matrix (i.e. positive or negative realness). Then, it is desired to prove that the sandwiched matrix 
in (7.28) is negative real. Expanding this sandwiched matrix using the definitions in (7.22) and (7.27) gives

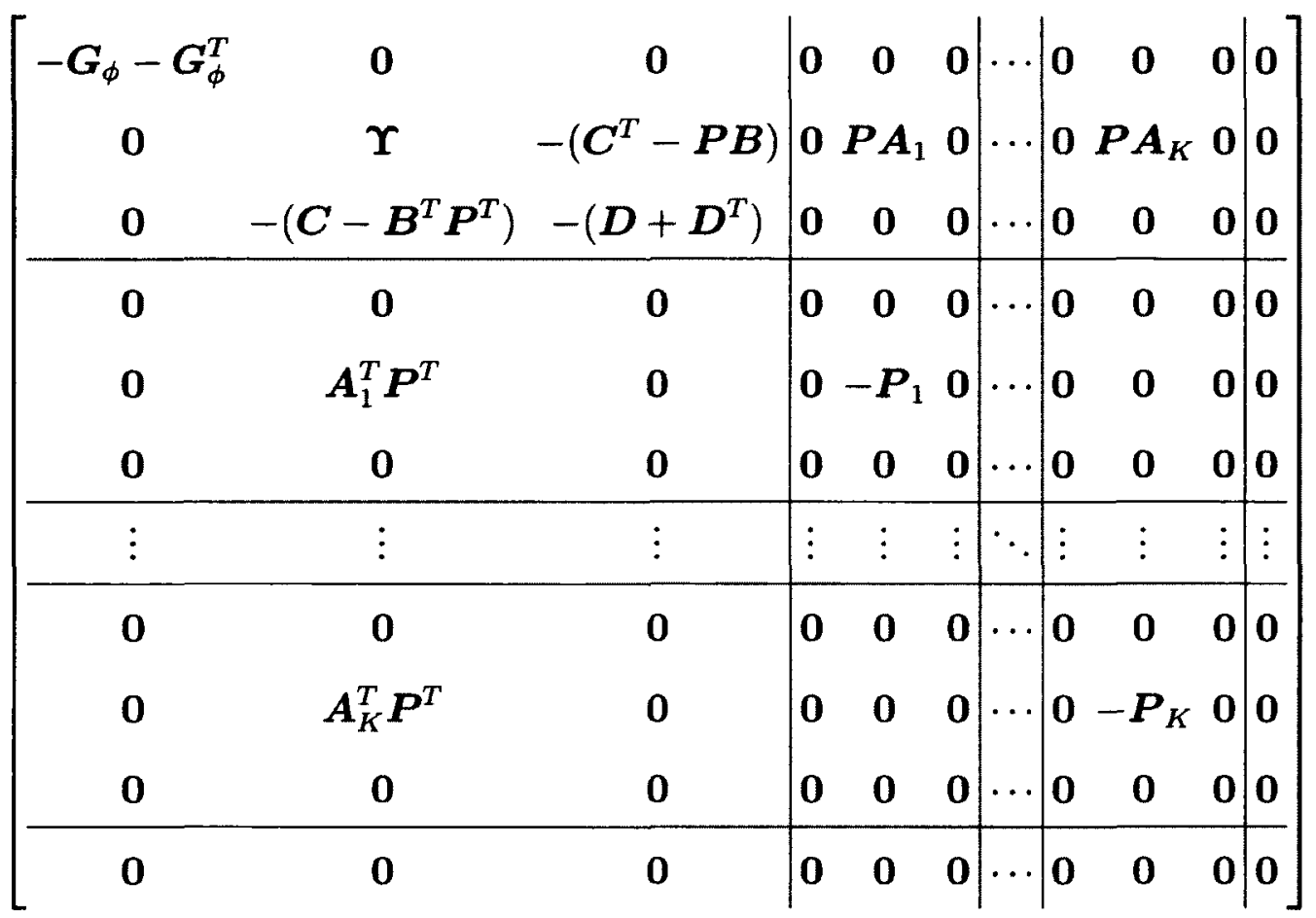

where $\boldsymbol{\Upsilon}=\boldsymbol{P} \boldsymbol{A}+\boldsymbol{A}^{T} \boldsymbol{P}^{T}+\sum_{k=1}^{K} \boldsymbol{P}_{k}$. Recall that interchanging row $i$ with row $j$, and subsequently interchanging column $i$ with column $j$ can be achieved via a congruence transform. Repeatedly interchanging rows and columns in this manner yields

$$
\boldsymbol{T}^{T}\left[\begin{array}{cr|ccccc}
-G_{\phi}-G_{\phi}^{T} & \mathbf{0} & \mathbf{0} & \mathbf{0} & \mathbf{0} & \mathbf{0} & \mathbf{0} \\
\mathbf{0} & \mathbf{0} & \mathbf{0} & \mathbf{0} & \mathbf{0} & \mathbf{0} & \mathbf{0} \\
\hline \mathbf{0} & \mathbf{0} & \boldsymbol{\Upsilon} & \boldsymbol{P} \boldsymbol{A}_{1} & \cdots & \boldsymbol{P} \boldsymbol{A}_{K} & -\left(C^{T}-\boldsymbol{P B}\right) \\
\mathbf{0} & \mathbf{0} & \boldsymbol{A}_{1}^{T} \boldsymbol{P}^{T} & -\boldsymbol{P}_{1} & \cdots & \mathbf{0} & \mathbf{0} \\
\mathbf{0} & \mathbf{0} & \vdots & \vdots & \ddots & \vdots & \vdots \\
\mathbf{0} & \mathbf{0} & \boldsymbol{A}_{K}^{T} \boldsymbol{P}^{T} & \mathbf{0} & \cdots & -\boldsymbol{P}_{K} & \mathbf{0} \\
\mathbf{0} & \mathbf{0} & -\left(\boldsymbol{C}-\boldsymbol{B}^{T} \boldsymbol{P}^{T}\right) & \mathbf{0} & \cdots & \mathbf{0} & -\left(\boldsymbol{D}+\boldsymbol{D}^{T}\right)
\end{array}\right] \boldsymbol{T}
$$


where $\mathcal{T}$ is the overall row/column transformation matrix. From the original MNA formulation for the RLC network, $\left(\boldsymbol{G}_{\phi}+\boldsymbol{G}_{\phi}^{T}\right) \geq \mathbf{0}$ and $-\left(\boldsymbol{G}_{\phi}+\boldsymbol{G}_{\phi}^{T}\right) \leq \mathbf{0}$. Next, note that the bottom quadrant of (7.31) is equivalent to the matrix $\boldsymbol{\Omega} \leq \mathbf{0}$ in (7.10b), which the embedded descriptor system satisfies by construction. Therefore, it is clear that $(7.25 \mathrm{~b})$ is satisfied and this completes the proof.

\subsection{Numerical Results}

In this section, two examples are presented to demonstrate the accuracy and efficiency of performing unified transient analysis using the proposed passive model-order reduction algorithm. All the examples are run on an Intel $i 7-9202.67 \mathrm{GHz}$ processor with $12 \mathrm{~Gb}$ of RAM.

\subsubsection{Example 1}

To validate the accuracy and efficiency of the proposed algorithm, it is tested on a large RLC circuit containing an embedded subnetwork described by a TDD system. The overall system consists of a large two-port RLC circuit with 7200 resistors, 2400 capacitors, and 2400 inductors connected with a two-port tabulated data subnetwork modeled using delayed rational functions [30] (as shown in Fig. 7.2). The embedded subnetwork was found to be passive using the proposed passivity verification technique in Section 7.3.1 and is of size 48 with 6 unique delays. The overall size of the original system, consisting of the RLC network and the embedded tabulated data subnetwork, is 4857 . Since the original network is an interconnection of passive components, it is passive.

Next, a conventional congruence transform-based Krylov-subspace model-order re- 


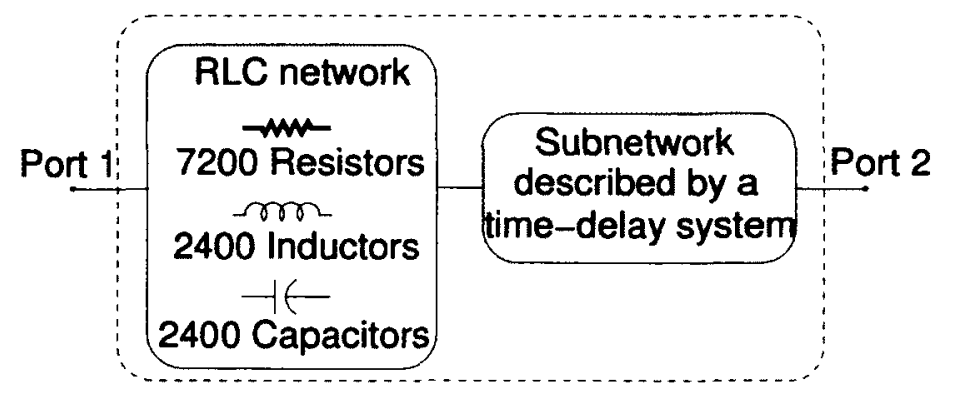

FIGURE 7.2: Original network for example 1.

duction procedure is applied directly to the overall original network to obtain a reduced model of size 33 (referred to as the conventional model). Fig. 7.3 shows a sample accuracy comparison of the admittance parameters, $\boldsymbol{Y}_{22}(s)$, between 0 and 1.5 GHz. It is clear that the original system and the reduced model are in good agreement. An application of Theorem 7.1 on the reduced model failed to meet the sufficient passivity conditions as given by the linear matrix inequalities in (7.10). The passivity of the reduced model was also cross-verified by performing a frequency-sweep and found to be nonpassive. Fig. 7.4 shows the resulting eigenvalues for the original and conventional reduced models along the imaginary axis. As seen, the conventional reduced model has negative eigenvalues after $3 \mathrm{GHz}$ indicating that it is not passive.

Next, the proposed passive model-order reduction algorithm is applied on the original network. First, the transformation matrix $\boldsymbol{P}$ was found by solving the linear matrix inequalities in (7.10). Then, the network was transformed following the process detailed in Section 7.3.2 and subsequently, embedded in the RLC network. Next, following the proposed reduction procedure as outlined in Section 7.3.3, the overall network was reduced to a system of size 33. As seen from Fig. 7.3, the admittance parameters of the proposed model accurately match the original network. A solution to the linear matrix inequalities in Theorem 7.1 was found, indicating that the 


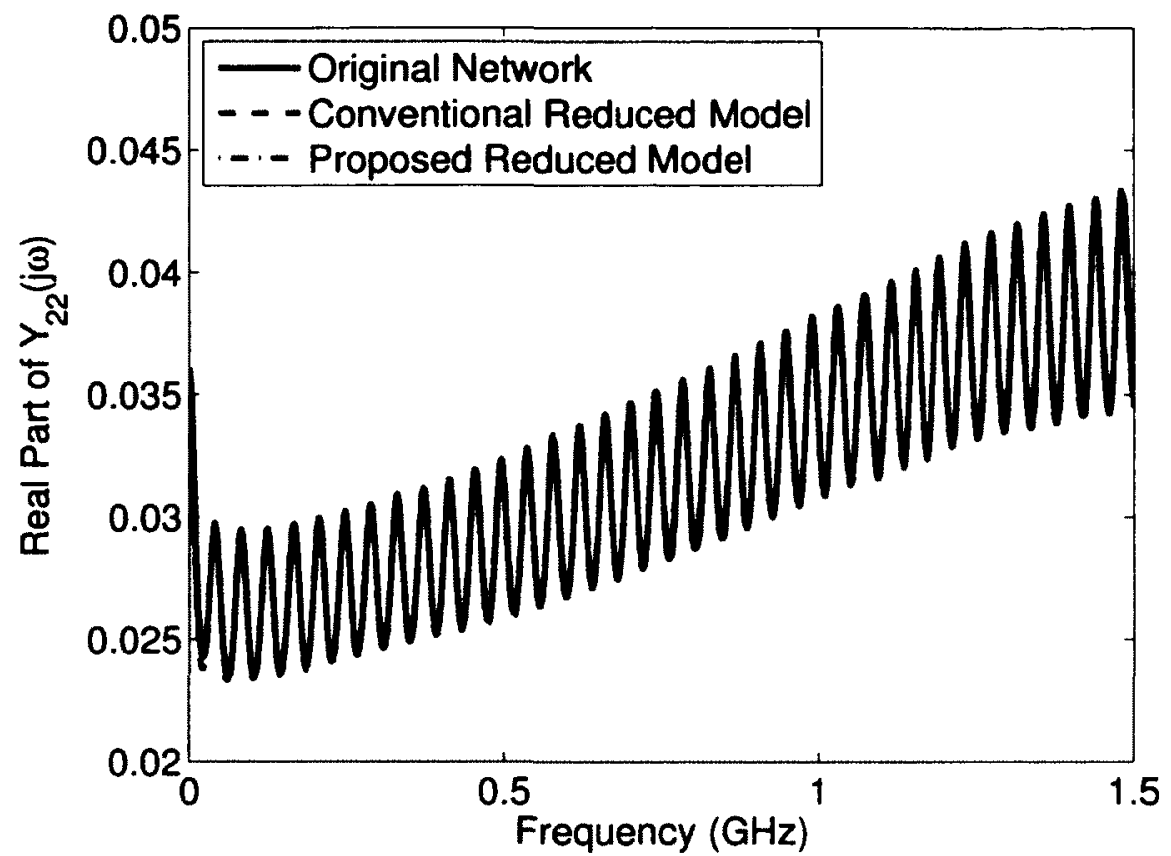

FIGURE 7.3: A sample spectrum of admittance parameters for example 1 (all the responses overlap).

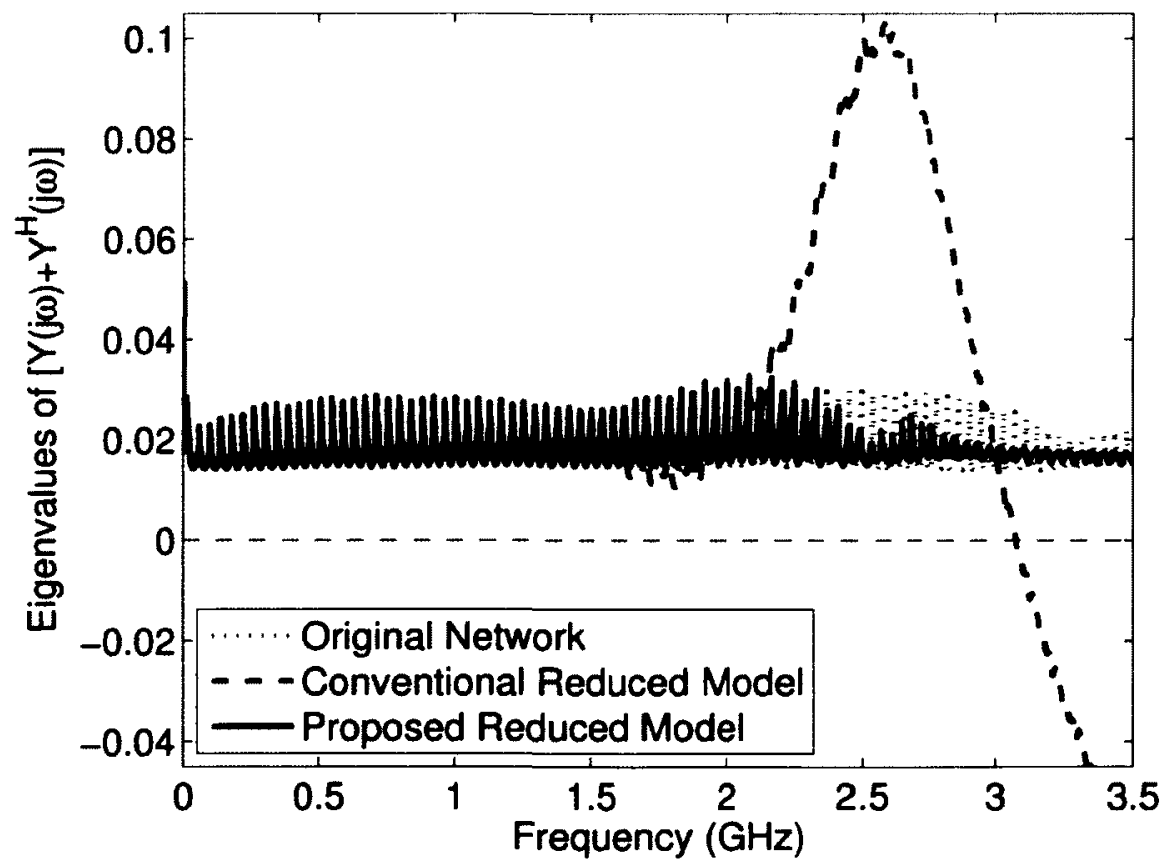

FIGURE 7.4: Eigenvalue spectrum of admittance parameters for example 1. 


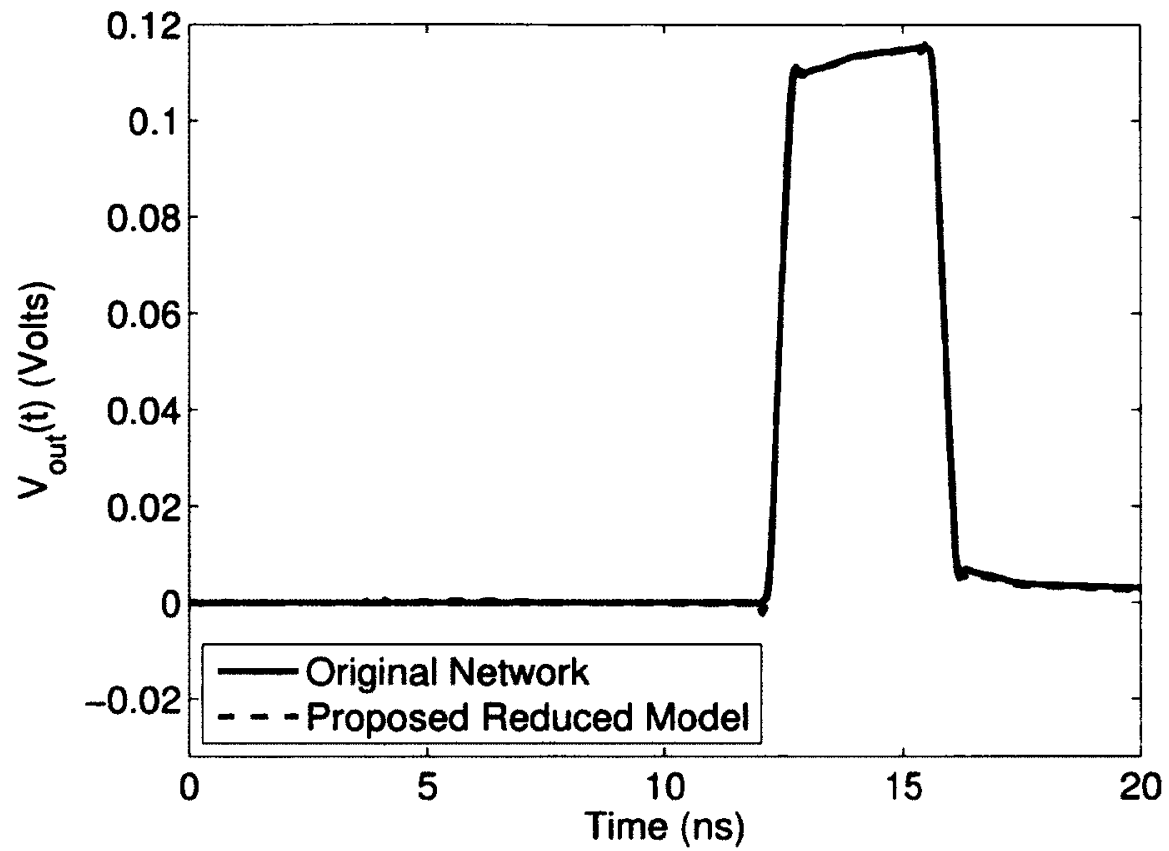

FIGURE 7.5: A comparison of transient responses for example 1.

reduced-order model is passive. In addition, this result is cross-verified by performing a robust frequency-sweep of the eigenvalues. As can be seen from Fig. 7.4, the proposed model does not contain any negative eigenvalues, implying that it is passive.

The transient performance of the original and proposed reduced model was also compared in HSPICE. Each model was excited by a trapezoidal pulse with $0.5 \mathrm{~ns}$ rise/fall times and a $3 \mathrm{~ns}$ pulse width. As seen in Fig. 7.5, the transient responses from the original and proposed models are in good agreement. For transient analysis, the original and proposed models required 10.65 and 1.84 seconds, respectively.

\subsubsection{Example 2}

In this example, the proposed algorithm is tested on a large four-port RLC circuit containing a embedded subnetworks described by TDD systems. An illustration of 


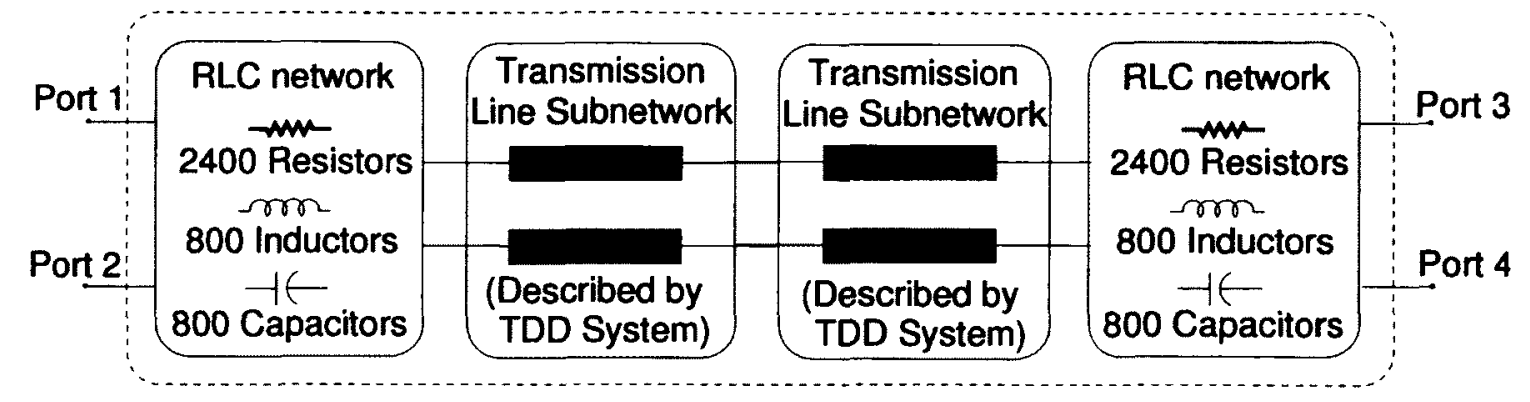

FIGURE 7.6: Original network for example 2.

this network is shown in Fig. 7.6. The overall system consists of a cascade of a large RLC circuit, 2 four-port transmission line networks, and another large RLC circuit. The transmission lines are each modeled using the method of characteristics [101]. The size of the original system is 3330 with 3 unique delays. The embedded subnetwork is found to be passive using the proposed passivity verification technique. Consequently, the original network is passive.

Next, the conventional and proposed congruence transform-based model-order reduction algorithms are applied to the original network. Both the conventional and proposed reduced-order models obtained are of size 38. Fig. 7.7 shows a sample accuracy comparison of the admittance parameters, $\boldsymbol{Y}_{42}(s)$, between 0 and $3.5 \mathrm{GHz}$. It is clear that the responses of the original, conventional, and proposed systems are all in good agreement.

Next, the passivity of the reduced-order macromodels is verified. An application of Theorem 7.1 on the conventional reduced-order model failed to meet the sufficient passivity conditions as given by the linear matrix inequalities in (7.10). The passivity of the conventional model is also cross-verified by performing a frequency-sweep and found to be nonpassive. Fig. 7.8 shows the resulting eigenvalues for the original and conventional reduced models along the imaginary axis. As seen, the conventional 


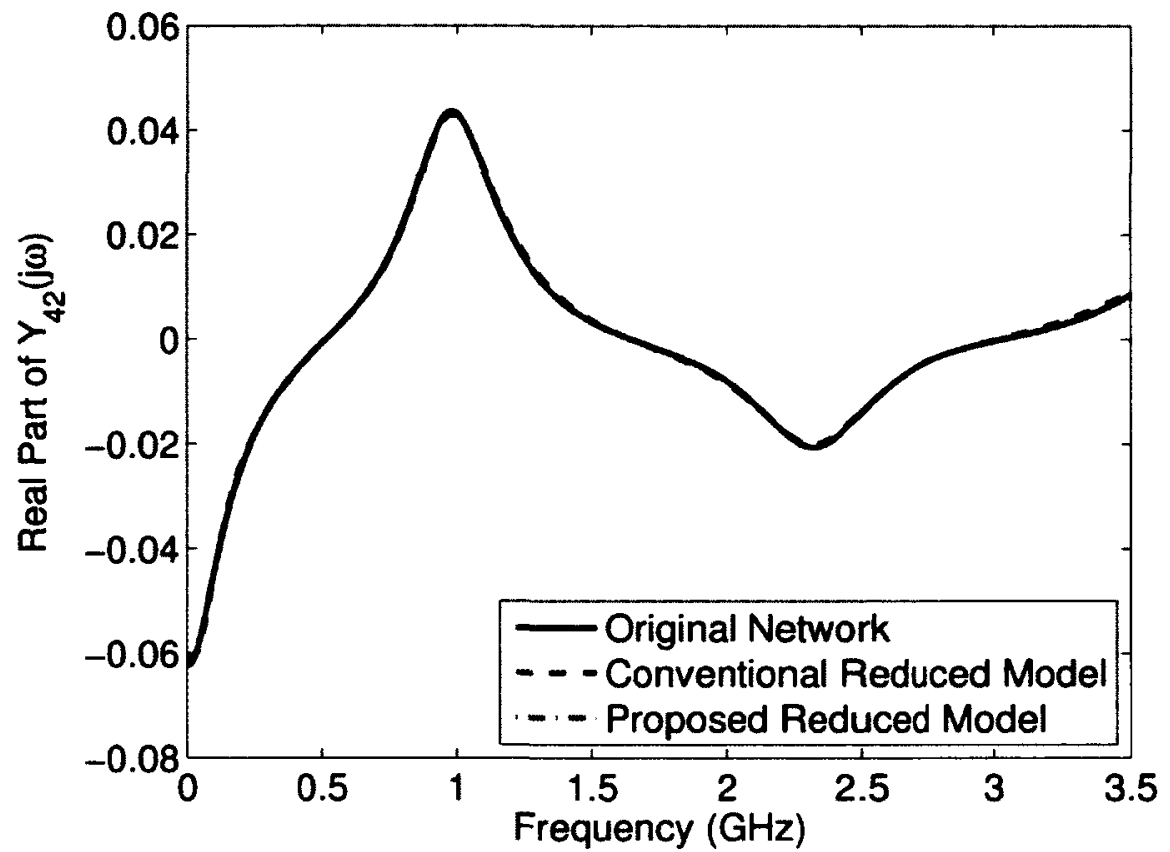

FIGURE 7.7: A sample spectrum of admittance parameters for example 2 (all the responses overlap).

reduced model has negative eigenvalues after $8 \mathrm{GHz}$ indicating that it is not passive. On the other hand, the proposed reduced-order model is found to be passive using Theorem 7.1. In addition, this result is cross-verified by performing a robust frequency-sweep of the eigenvalues. As can be seen from Fig. 7.8, the proposed model does not contain any negative eigenvalues, implying that it is passive.

To verify the accuracy of the proposed model in the time-domain, the original and proposed model are simulated in HSPICE. Each model is excited by a trapezoidal pulse with $0.15 \mathrm{~ns}$ rise/fall times and a 3 ns pulse width. As seen in Fig. 7.9, the transient responses from the original and proposed models are in good agreement. The transient simulation times for the original and proposed models were 12.98 and 2.77 seconds, respectively. 


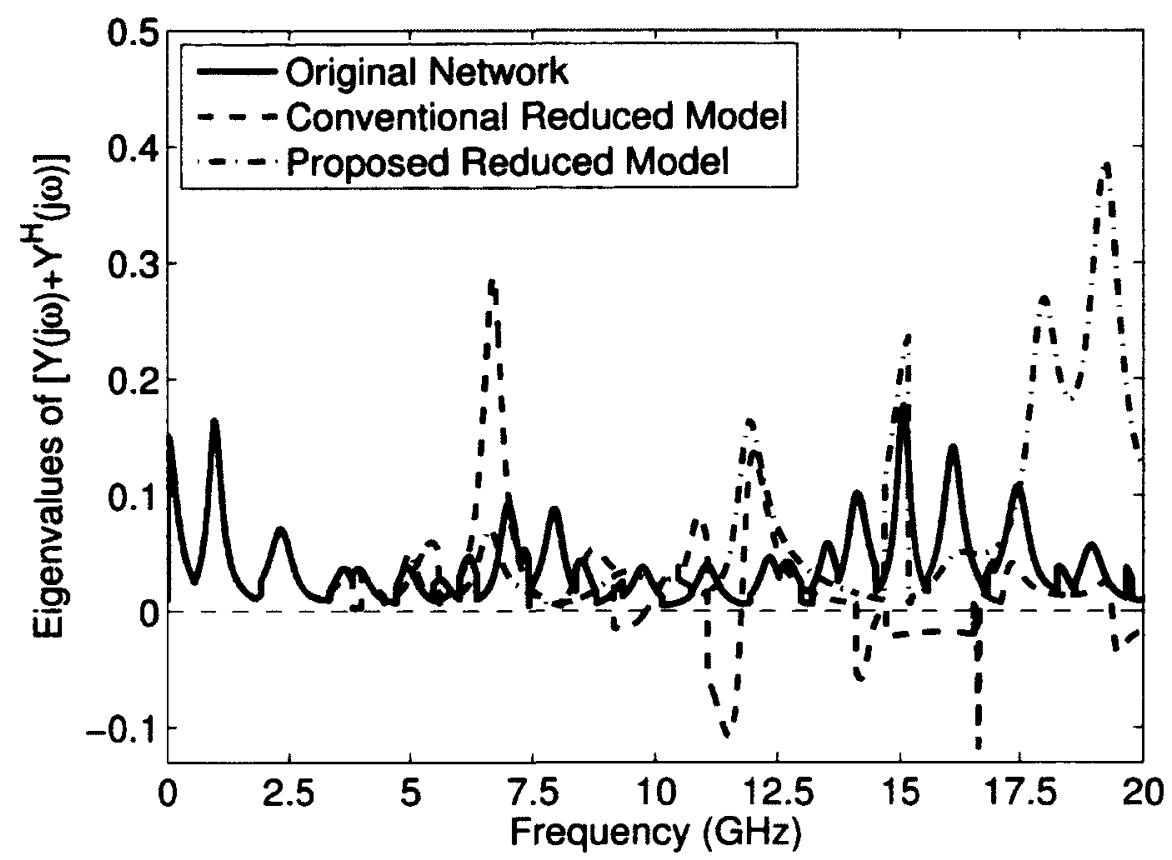

FIGURE 7.8: A sample eigenvalue spectrum of admittance parameters for example 2.

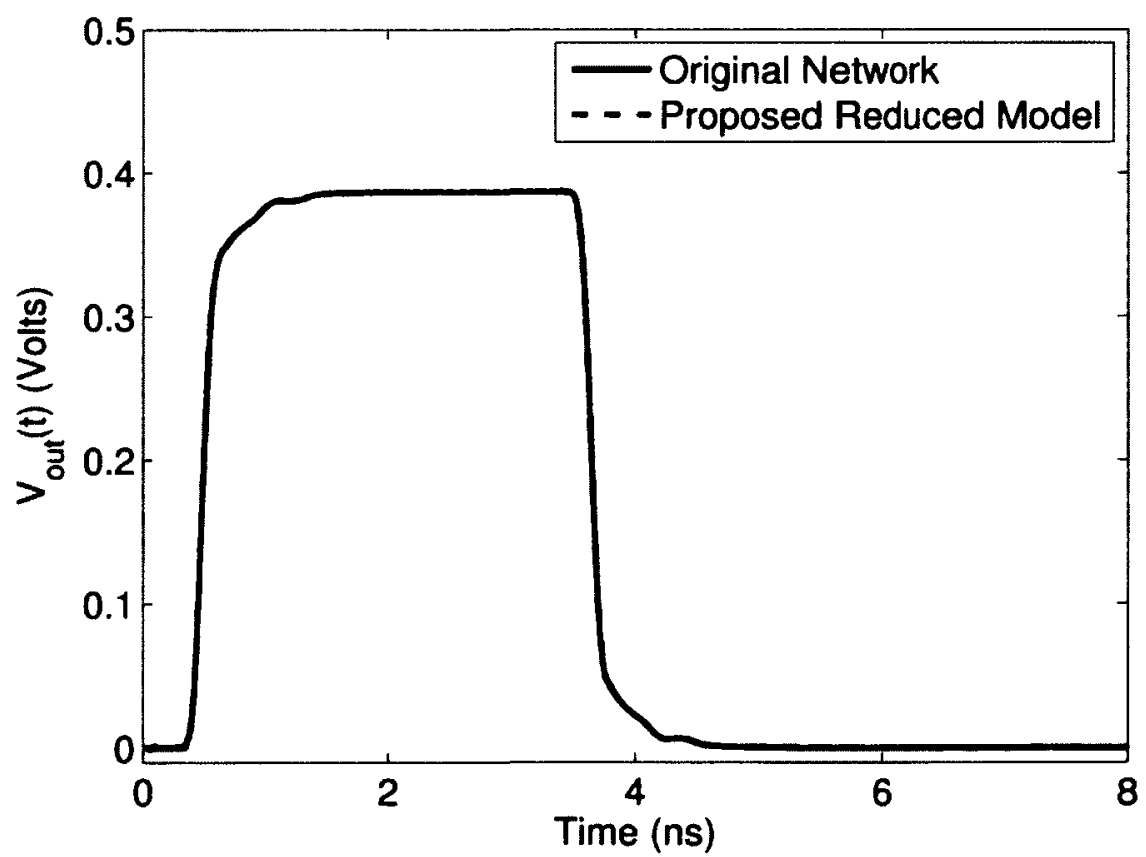

FIGURE 7.9: A comparison of transient responses for example 2. 


\subsection{Summary}

In this chapter, a new passive model-order reduction methodology has been presented for RLC circuits with embedded subnetworks described by general TDD systems. The proposed algorithm enables global and passive reduction of the entire RLC network along with its embedded subnetworks in a single step. In addition, a new passivity verification algorithm has been presented for general TDD systems with multiple time-delays. Numerical results demonstrating efficient and accurate analysis using the proposed algorithms were also presented. 


\section{CHAPTER 8}

\section{Conclusions and Future Research}

\subsection{Summary}

In this thesis, new algorithms for incorporating electrically long networks characterized by tabulated data into standard circuit simulators have been presented. The proposed algorithms address various issues such as delay-extraction techniques, Delayed Rational Function (DRF) approximations, macromodel stability, macromodel passivity verification and quantification of passivity violation regions, passivity enforcement of DRF macromodels, and passive delay-based model-order reduction. A brief summary of these algorithms and their practical benefits is presented below.

1) Passivity verification algorithms are developed for DRF-based macromodels obtained from tabulated scattering [68]-[71] and admittance [67] parameter data. In this thesis, passivity verification was formulated as a Frequency-Dependent Generalized Eigenvalue Problem (FDGEP). The proposed FDGEPs are quasiperiodic and consequently, need only be solved over a single period correspond- 
ing to a finite interval along the imaginary axis, yielding significant speed-up compared to conventional (direct) passivity verification techniques.

2) Passivity enforcement algorithms have been developed for nonpassive DRFbased macromodels in the scattering and admittance domains. The proposed algorithms iteratively perturb the residues of a nonpassive macromodel until it becomes passive [67], [68].

3) The proposed DRF passivity verification and compensation algorithms [67]-[71] are the first methods available addressing passive DRF macromodeling. Numerous publications relying on the proposed DRF passivity verification schemes, and building on the results of the proposed DRF passivity enforcement scheme can be found in the literature.

4) The proposed passivity verification and compensation techniques can easily be automated, providing a methodology to implement passive DRF macromodels in software packages for commercial distribution.

5) For DRF-based macromodels with long delays and dominant periodic reflections, a new DRF-based formulation is developed that uses only a single delay term in the denominator to accurately capture all the delayed rational terms corresponding to a single, dominant periodic reflection [72]. The delays are identified using time-frequency analysis and subsequently, an iterative least squares technique is used to obtain the poles and residues in the approximation. For stability of DRF formulations with a single time-delay in the denominator, a new theorem was presented. Using the proposed theorem, an efficient methodology was developed to guarantee the stability of the DRF approximation. For 
transient analysis, the proposed DRF-based macromodel (with delays in the denominator) is shown to provide considerable speed-up compared to conventional DRF-based macromodeling techniques with all the delays in the numerators.

6) For passivity verification of TDD systems, a new theorem was presented. The proposed theorem formulates passivity verification of TDD systems as a solution of linear matrix inequalities, which can be solved efficiently using interior point methods.

7) A passive model-order reduction algorithm is developed for RLC networks with embedded subnetworks described by TDD systems. The proposed algorithm formulates a unified set of equations for the overall circuit such that, reducedorder models obtained via congruence transform-based model-order reduction techniques preserve the passivity of the original network. The new method is capable of incorporating multiple embedded subnetworks described by TDD systems.

\subsection{Future Research}

As discussed in the previous section, this thesis presented novel contributions addressing passivity issues for the specific case of DRF-based macromodels obtained from tabulated data in the scattering and admittance domain. The proposed algorithms address various issues such as delay-extraction techniques, DRF approximations, macromodel stability, macromodel passivity verification and quantification of passivity violation regions, passivity enforcement of DRF macromodels, and passive delay-based model-order reduction. This thesis not only led to numerous publications, 
but has also laid the seed for several current graduate thesis projects. In addition, it has the potential for further investigation into several open research problems, a few of them are listed below.

1) Stable, Efficient DRF Macromodels for Networks with Multiple Periodic Delays: In Chapter 6, preliminary results for stable macromodeling tabulated data networks with a single dominant periodic delay was presented. In many cases, high-speed modules may contain several periodic delays. Further research is required to investigate the extension of the proposed algorithms to tabulated networks with multiple dominant periodic delays. This has immediate applications in optoelectronic circuits and metamaterial based designs.

2) Passivity Compensation of DRF Macromodels with Delays in the Denominator: In Chapter 6, a new macromodeling technique was presented for tabulated data networks with periodic reflections. However, these DRF macromodels are not guaranteed to be passive. In addition, since there are delays in the denominator as well as the numerator, the passivity verification and compensation techniques presented in Chapter 4 and 5 are not directly applicable.

3) Delay-Dependent Passivity Verification of TDD Systems: In chapter 7, a passivity verification technique was developed for general TDD systems based on linear matrix inequalities. However, the proposed linear matrix inequalities only provide sufficient passivity conditions that are independent of the value of the time-delays. Further research can be explored to develop less conservative delay-dependent passivity criteria for TDD systems. 
4) Passive Model-Order Reduction of TDD Systems via Poor Man's Truncated Balanced Realization (PMTBR): In chapter 7, a new congruence transform based model-order reduction algorithm was introduced that guaranteed the passivity of the resulting reduced-order macromodel. Further approaches such as PMTBR can be explored for achieving the same goal. 


\section{Bibliography}

[1] A. Deutsch, "Electrical characteristics of interconnections for high-performance systems," Proceedings of the IEEE, vol. 86, no. 2, pp. 315-355, February 1998.

[2] C. R. Paul, Analysis of multiconductor transmission lines. New York, NY: John Wiley and Sons Inc., 1994.

[3] E. Bogatin, Signal Integrity-Simplified, ser. Prentice Hall Modern Semiconductor Design Series. Upper Saddle River, New Jersey: Prentice Hall, 2004.

[4] S. H. Hall, G. W. Hall, and J. A. McCall, High-Speed Digital System Design: A Handbook of Interconnect Theory and Design Practices. John-Wiley and Sons, Inc., 2000 .

[5] R. Achar and M. S. Nakhla, "Simulation of high-speed interconnects," Proceedings of the IEEE, vol. 89, no. 5, pp. 693-728, May 2001.

[6] P. Gunupudi, T. Smy, J. Klein, and Z. J. Jakubczyk, "Self-consistent simulation of opto-electronic circuits using a modified nodal analysis formulation," IEEE Transactions on Advanced Packaging, vol. 33, no. 4, pp. 979-993, November 2010. 
[7] L. Pillage and R. A. Rohrer, "Asymptotic waveform evaluation for timing analysis," IEEE Transactions on Computer-Aided Design of Integrated Circuits and Systems, vol. 9, no. 4, pp. 352-366, April 1990.

[8] A. Odabasioglu, M. Celik, and L. Pileggi, "PRIMA : Passive reduced-order interconnect macromodeling algorithm," IEEE Transactions on Computer-Aided Design of Integrated Circuits and Systems, vol. 17, no. 8, pp. 645-654, August 1998.

[9] A. R. Djordjević, T. K. Sarkar, and R. F. Harrington, "Analysis of lossy transmission lines with arbitrary nonlinear terminal networks," IEEE Transactions on Microwave Theory and Techniques, vol. MTT-34, no. 6, pp. 660-666, June 1986.

[10] J. E. Schutt-Aine and R. Mittra, "Scattering parameter transient analysis of transmission lines loaded with nonlinear terminations," IEEE Transactions on Microwave Theory and Techniques, vol. 36, no. 3, pp. 529-536, March 1988.

[11] D. Winklestein, M. B. Steer, and R. Pomerleau, "Simulation of arbirtrary transmission line networks with nonlinear terminations," IEEE Transactions on Circuits and Systems, vol. 38, no. 4, pp. 418-422, April 1991.

[12] S. Lin and E. S. Kuh, "Transient simulation of lossy interconnects based on the recursive convolution formulation," IEEE Transactions on Circuits and Systems-Part I: Fundamental Theory and Applications, vol. 39, no. 11, pp. 879-892, November 1992. 
[13] C. Sanathanan and J. Koerner, "Transfer function synthesis as a ratio of two complex polynomials," IEEE Transactions on Automatic Control, vol. 8, no. 1, pp. 56-58, January 1963.

[14] W. Beyene and J. Schutt-Aine, "Interconnect simulation using order reduction and scattering parameters," IEEE Electronic Components and Technology Conference, pp. 627-631, May 1998.

[15] —, "Efficient transient simulation of high-speed interconnects characterized by sampled data," IEEE Transactions on Components, Packaging and Manufacturing Technology-Part B: Advanced Packaging, vol. 21, pp. 105-114, February 1998.

[16] C. P. Coelho, J. R. Phillips, and L. M. Silveira, "Robust rational function approximation algorithm for model generation," Proceedings of the IEEE Design Automation Conference, pp. 207-212, June 1999.

[17] B. Gustavsen and A. Semlyen, "Rational approximation of frequency domain responses by vector fitting," IEEE Transactions on Power Delivery, vol. 14, no. 3, pp. 1052-1061, July 1999.

[18] B. Gustavsen, "Improving the pole relocating properties of vector fitting," IEEE Transactions on Power Delivery, vol. 21, no. 3, pp. 1587 - 1592, July 2006.

[19] S. Grivet-Talocia, "Package macromodeling via time-domain vector fitting," IEEE Microwave and Wireless Components Letters, vol. 13, no. 11, pp. 472 474, November 2003. 
[20] D. Deschrijver, B. Haegeman, and T. Dhaene, "Orthonormal vector fitting: A robust macromodeling tool for rational approximation of frequency domain responses," IEEE Transactions on Advanced Packaging, vol. 30, no. 2, pp. 216225, May 2007.

[21] W. Hendrickx and T. Dhaene, "A discussion of "rational approximation of frequency domain responses by vector fitting"," IEEE Transactions on Power Delivery, vol. 21, no. 1, pp. 441-443, February 2006.

[22] G. Antonini, D. Deschrijver, and T. Dhaene, "A comparative study of vector fitting and orthonormal vector fitting techniques for EMC applications," Proceedings of the IEEE International Symposium on Electromagnetic Compatibility, vol. 1, pp. 6-11, August 2006.

[23] B. Nouri, R. Achar, and M. S. Nakhla, " $z$-domain orthonormal basis functions for physical system identifications," IEEE Transactions on Advanced Packaging, vol. 33, no. 1, pp. 293-307, February 2010.

[24] S. Grivet-Talocia and M. Bandinu, "Improving the convergence of vector fitting for equivalent circuit extraction from noisy frequency responses," IEEE Transactions on Electromagnetic Compatibility, vol. 48, no. 1, pp. 104-120, February 2006.

[25] S. Lefteriu and A. C. Antoulas, "A new approach to modeling multiport systems from frequency-domain data," IEEE Transactions on Computer-Aided Design of Integrated Circuits and Systems, vol. 29, no. 1, pp. 14-27, January 2010.

[26] A. Charest, D. Saraswat, M. Nakhla, R. Achar, and N. Soveiko, "Compact macromodeling of high-speed circuits via delayed rational functions," IEEE 
Microwave and Wireless Components Letters, vol. 17, no. 12, pp. 828-830, December 2007.

[27] A. Charest, M. S. Nakhla, R. Achar, D. Saraswat, N. Soveiko, and I. Erdin, "Time domain delay extraction-based macromodeling algorithm for long-delay networks," IEEE Transactions on Advanced Packaging, vol. 33, no. 1, pp. 219 235, February 2010.

[28] S. Grivet-Talocia, "Delay-based macromodels for long interconnects via timefrequency decompositions," Proceedings of the IEEE Workshop on Electrical Performance of Electronic Packaging, pp. 199-202, October 2006.

[29] A. Chinea, P. Triverio, and S. Grivet-Talocia, "Compact macromodeling of electrically long interconnects," Proceedings of the IEEE Workshop on Electrical Performance of Electronic Packaging, pp. 199-202, October 2008.

[30] —, "Delay-based macromodeling of long interconnects from frequencydomain terminal responses," IEEE Transactions on Advanced Packaging, vol. 33 , no. 1, pp. 246-256, February 2010.

[31] R. Mandrekar and M. Swaminathan, "Delay extraction from frequency domain data for causal macro-modeling of passive networks," Proceedings of the IEEE International Symposium on Circuits and Systems, vol. 6, pp. 5758-5761, May 2005.

[32] R. Mandrekar, K. Srinivasan, E. Engin, and M. Swaminathan, "Co-simulation of signal and power delivery networks with causality," Proceedings of the IEEE Workshop on Electrical Performance of Electronic Packaging, pp. 337-340, October 2005 . 
[33] R. Mandrekar and M. Swaminathan, "Causality enforcement in transient simulation of passive networks through delay extraction," Proceedings of the IEEE Workshop on Signal Propagation on Interconnects, pp. 25-28, May 2005.

[34] R. Mandrekar, K. Srinivasan, E. Engin, and M. Swaminathan, "Causality enforcement in transient co-simulation of signal and power delivery networks," IEEE Transactions on Advanced Packaging, vol. 30, no. 2, pp. 270-278, May 2007.

[35] S. Boyd, V. Balakrishnan, and P. Kabamba, "A bisection method for computing the $\mathrm{H}_{\infty}$ norm of a transfer matrix and related problems," Mathematics of Control, Signals, and Systems, vol. 2, no. 3, pp. 207-219, September 1989.

[36] B. Gustavsen and A. Semlyen, "Fast passivity assessment for S-parameter rational models via a half-size test matrix," IEEE Transactions on Microwave Theory and Techniques, vol. 56, no. 12, pp. 2701-2708, December 2008.

[37] A. Semlyen and B. Gustavsen, "A half-size singularity test matrix for fast and reliable passivity assessment of rational models," IEEE Transactions on Power Delivery, vol. 24, no. 1, pp. 345-351, January 2009.

[38] S. Grivet-Talocia, "On passivity characterization of symmetric rational macromodels," IEEE Transactions on Microwave Theory and Techniques, vol. 58, no. 5, pp. 1238-1247, May 2010.

[39] D. Saraswat, R. Achar, and M. S. Nakhla, "A fast algorithm and practical considerations for passive macromodeling of measured/simulated data," IEEE Transactions on Advanced Packaging, vol. 27, no. 1, pp. 57-70, February 2004. 
[40] _ - "Global passivity enforcement algorithm for macromodels of interconnect subnetworks characterized by tabulated data," IEEE Transactions on Very Large Scale Integration (VLSI) Systems, vol. 13, no. 7, pp. 819-832, July 2005.

[41] — - "Fast passivity verification and enforcement via reciprocal systems for interconnects with large order macromodels," IEEE Transactions on Very Large Scale Integration (VLSI) Systems, vol. 15, no. 1, pp. 48-59, January 2007.

[42] D. Saraswat, "Global compact passive macromodeling algorithms for high-speed circuits," Ph.D. dissertation, Carleton University, 2006.

[43] S. Grivet-Talocia, "Passivity enforcement via perturbation of hamiltonian matrices," IEEE Transactions on Circuits and Systems-Part I: Regular Papers, vol. 51, no. 9, pp. 1755-1769, September 2004.

[44] S. Grivet-Talocia and A. Ubolli, "On the generation of large passive macromodels for complex interconnect structures," IEEE Transactions on Advanced Packaging, vol. 29, no. 1, pp. 39-54, February 2006.

[45] _ , "Passivity enforcement with relative error control," IEEE Transactions on Microwave Theory and Techniques, vol. 55, no. 11, pp. 2374-2383, November 2007.

[46] B. Gustavsen and A. Semlyen, "Enforcing passivity for admittance matrices approximated by rational functions," IEEE Transactions on Power Systems, vol. 16, no. 1, pp. 97-104, February 2001. 
[47] B. Gustavsen, "Passivity enforcement of rational models via modal perturbation," IEEE Transactions on Power Delivery, vol. 23, no. 2, pp. 768-775, April 2008.

[48] C. P. Coelho, J. R. Phillips, and L. M. Silveira, "Passive constrained rational approximation algorithm using nevanlinna-pick interpolation," Proceedings of the IEEE Design, Automation and Test in Europe, pp. 923 - 930, March 2002.

[49] — - "A convex programming approach for generating guaranteed passive approximations to tabulated frequency-data," IEEE Transactions on ComputerAided Design of Integrated Circuits and Systems, vol. 23, no. 2, pp. 293-301, February 2004.

[50] E. Gad, C. Chen, M. Nakhla, and R. Achar, "Passivity verification in delaybased macromodels of electrical interconnects," IEEE Transactions on Circuits and Systems--Part I: Regular Papers, vol. 52, no. 10, pp. 2173-2187, October 2005.

[51] C. Chen, E. Gad, M. Nakhla, and R. Achar, "Passivity verification in delaybased macromodels of multiconductor electrical interconnects," IEEE Transactions on Advanced Packaging, vol. 30, no. 2, pp. 246-256, May 2007.

[52] A. Chinea and S. Grivet-Talocia, "Perturbation schemes for passivity enforcement of delay-based transmission line macromodels," IEEE Transactions on Advanced Packaging, vol. 31, no. 3, pp. 568-578, August 2008.

[53] C. Chen, D. Saraswat, R. Achar, E. Gad, M. Nakhla, and M. C. E. Yagoub, "Passivity compensation algorithm for method-of-characteristics based multiconductor transmission line interconnect macromodels," IEEE Transactions on 
Very Large Scale Integration (VLSI) Systems, vol. 17, no. 8, pp. 1061-1072, August 2009.

[54] B. Gustavsen, "Time delay identification for transmission line modeling," Proceedings of the IEEE Workshop on Signal Propagation on Interconnects, pp. 103-106, May 2004.

[55] L. D. Tommasi and B. Gustavsen, "Low order transmission line modeling by modal decomposition and minimum phase shift fitting," Proceedings of the IEEE Workshop on Signal Propagation on Interconnects, pp. 89-92, May 2006.

[56] A. Charest, M. Nakhla, and R. Achar, "Passive macromodeling of transmission line type interconnects characterized by tabulated data," Proceedings of the IEEE North-East Workshop on Circuits and Systems, pp. 1421-1424, August 2007.

[57] A. Charest, M. Nakhla, R. Achar, and I. Erdin, "Delay extraction-based passive macromodeling techniques for transmission line type interconnects characterized by tabulated multiport data," Analog Integrated Circuits and Signal Processing, vol. 60, no. 1, pp. 13-25, August 2009.

[58] B. Gustavsen, "Fast delay-less interconnect macromodeling and simulation by assumption of a constant eigenvector matrix," Proceedings of the IEEE Workshop on Signal Propagation on Interconnects, pp. 1-4, May 2008.

[59] P. Triverio, S. Grivet-Talocia, and A. Chinea, "Black-box identification of delaybased macromodels from measured terminal responses," Proceedings of the IEEE Workshop on Signal Propagation on Interconnects, pp. 1-4, May 2009. 
[60] _ - "Identification of highly efficient delay-rational macromodels of long interconnects from tabulated frequency data," IEEE Transactions on Microwave Theory and Techniques, vol. 58, no. 3, pp. 566-577, March 2010.

[61] E. Chiprout and M. S. Nakhla, "Analysis of interconnect networks using complex frequency hopping," IEEE Transactions on Computer-Aided Design of Integrated Circuits and Systems, vol. 14, no. 2, pp. 186-200, February 1995.

[62] J. R. Phillips, L. Daniel, and L. M. Silveira, "Guaranteed passive balancing transformations for model order reduction," IEEE Transactions on ComputerAided Design of Integrated Circuits and Systems, vol. 22, no. 8, pp. 1027-1041, August 2003.

[63] J. R. Phillips and L. M. Silveira, "Poor man's TBR: A simple model reduction scheme," IEEE Transactions on Computer-Aided Design of Integrated Circuits and Systems, vol. 24, no. 1, pp. 43-55, January 2005.

[64] D. Saraswat, R. Achar, and M. S. Nakhla, "Passive reduction algorithm for RLC interconnect circuits with embedded state-space systems (PRESS)," IEEE Transactions on Microwave Theory and Techniques, vol. 52, no. 9, pp. 22152226, September 2004.

[65] W. Tseng, C. Chen, E. Gad, M. Nakhla, and R. Achar, "Passive order reduction for RLC circuits with delay elements," IEEE Transactions on Advanced Packaging, vol. 30, no. 4, pp. 830-840, November 2007.

[66] X. Wang, Q. Wang, Z. Zhang, Q. Chen, and N. Wong, "Balanced truncation for time-delay systems via approximate gramians," Proceedings of the IEEE Asia and South Pacific Design Automation Conference, pp. 55-60, January 2011. 
[67] A. Charest, M. Nakhla, R. Achar, and C. Chen, "Passivity verification and enforcement of delayed rational function macromodels from networks characterized by tabulated data," Proceedings of the IEEE Workshop on Signal Propagation on Interconnects, pp. 1-4, May 2009.

[68] A. Charest, M. Nakhla, and R. Achar, "Scattering domain passivity verification and enforcement of delayed rational functions," IEEE Microwave and Wireless Components Letters, vol. 19, no. 10, pp. 605-607, October 2009.

[69] — , "Passivity verification and enforcement of delayed rational approximations from scattering parameter based tabulated data," Proceedings of the IEEE Workshop on Electrical Performance of Electronic Packaging, pp. 65-68, October 2009 .

[70] —, "Efficient passivity verification of delayed rational function macromodels from networks characterized by tabulated data," Proceedings of the IEEE Workshop on Signal Propagation on Interconnects, pp. 109-112, May 2010.

[71] A. Charest, M. Nakhla, R. Achar, and D. Saraswat, "Passivity verification of delayed rational function based macromodels of tabulated networks characterized by scattering parameters," IEEE Transactions on Components, Packaging, and Manufacturing Technology, vol. 1, no. 3, pp. 386-398, March 2011.

[72] A. Charest, M. Nakhla, and R. Achar, "Delay extracted stable rational approximations for tabulated networks with periodic reflections," IEEE Microwave and Wireless Components Letters, vol. 19, no. 12, pp. 768-770, December 2009. 
[73] _ _ "Passive model-order reduction of RLC circuits with embedded time-delay descriptor systems," Proceedings of the IEEE Workshop on Electrical Performance of Electronic Packaging, pp. 223-226, October 2011.

[74] HSPICE Signal Integrity Guide, Synopsys, March 2009, c-2009.03.

[75] J. Vlach and K. Singhal, Computer Methods for Circuit Analysis and Design. Van Nostrand Reinhold Company Inc., 1983.

[76] A. Whitfield, "Asymptotic behavior of transfer function synthesis methods," International Journal of Control, vol. 45, no. 3, pp. 1083-1092, 1987.

[77] L. N. Trefethen and D. Bau, Numerical Linear Algebra. SIAM: Society for Industrial and Applied Mathematics, 1997.

[78] R. Achar, P. K. Gunupudi, M. Nakhla, and E. Chiprout, "Passive interconnect reduction algorithm for distributed/measured networks," IEEE Transactions on Circuits and Systems-Part II: Analog and Digital Signal Processing, vol. 47, no. 4, pp. 287-301, April 2000.

[79] M. E. Van Valkenburg, Introduction to Modern Network Synthesis. NY: John Wiley \& Sons Inc., 1960.

[80] P. Triverio, S. Grivet-Talocia, M. S. Nakhla, F. Canavero, and R. Achar, "Stability, causality, and passivity in electrical interconnect models," IEEE Transactions on Advanced Packaging, vol. 30, no. 4, pp. 795-808, November 2007.

[81] K. Ogata, Modern Control Engineering, 3rd ed. Prentice-Hall, Inc, 1997.

[82] K. Gu, V. L. Kharitonov, and J. Chen, Stability of Time-Delay Systems. Birkhäuser: Boston, 2003. 
[83] M. R. Wohlers, Lumped and Distributed Passive Networks. Academic Press, Inc., 1969.

[84] D. C. Youla, "Physical realizability criteria," IRE Transactions on Circuit Theory, vol. 7, no. 5, pp. 50-68, August 1960.

[85] A. Dounavis, R. Achar, and M. S. Nakhla, "Efficient passive circuit models for distributed networks with frequency-dependent parameters," IEEE Transactions on Advanced Packaging, vol. 23, no. 3, pp. 382-392, August 2000.

[86] B. D. O. Anderson and S. Vongpanitlerd, Network Analysis and Synthesis. Prentice-Hall, Inc., 1973.

[87] S. Boyd, L. E. Ghaoui, E. Feron, and V. Balakrishnan, Linear Matrix Inequalities in Systems and Control Theory. SIAM: Society for Industrial and Applied Mathematics, 1994.

[88] D. Saraswat, R. Achar, and M. S. Nakhla, "On passivity enforcement for macromodels of S-parameter based tabulated subnetworks," Proceedings of the IEEE International Symposium on Circuits and Systems, vol. 4, pp. 3777 - 3780, May 2005.

[89] G. W. Stewart and J. Sun, Matrix Perturbation Theory. Boston, MA: Academic Press, Inc., 1990.

[90] T. Kailath, Linear Systems. Toronto:Prentice Hall, 1980.

[91] K. Zhou and J. C. Doyle, Essentials of Robust Control. Upper Saddle River, N.J.:Prentice Hall, 1998. 
[92] A. Charest, "Delay extraction based macromodeling techniques for tabulated/measured data," Master's thesis, Carleton University, 2007.

[93] A. V. Oppenheim and R. W. Schafer, Discrete-time Signal Processing, 2nd ed. Prentice Hall, 1999.

[94] S. Mallat, A Wavelet Tour of Signal Processing, 2nd ed. Academic Press, 1999.

[95] E. S. Kuh and R. A. Rohrer, Theory of Linear Active Networks. San Francisco, CA:Holden-Day, 1967.

[96] A. Jeffrey, Complex analysis and applications. Boca Raton, Florida: CRC Press, Inc., 1992.

[97] P. A. Parrilo, "Structured semidefinite programs and semialgebraic geometry methods in robustness and optimization," Ph.D. dissertation, California Institute of Technology, 2000.

[98] J. F. Sturm, "Using SeDuMi 1.02, a MATLAB toolbox for optimization over symmetric cones," Optimization Methods and Software, vol. 11, no. 1-4, pp. 625-653, 1999.

[99] J. Löfberg, "YALMIP: A toolbox for modeling and optimization in MATLAB," Proceedings of the IEEE International Symposium on Computer Aided Control Systems Design, pp. 284-289, September 2004.

[100] F.-Y. Chang, "The generalized method of characteristics for waveform relaxation analysis of lossy coupled transmission lines," IEEE Transactions on Microwave Theory and Techniques, vol. 37, no. 12, pp. 2028-2038, December 1989. 
[101] S. Grivet-Talocia, H.-M. Huang, A. E. Ruehli, F. Canavero, and I. M. Elfadel, "Transient analysis of lossy transmission lines: an efficient approach based on the method of characteristics," IEEE Transactions on Advanced Packaging, vol. 27, no. 1, pp. 45-46, February 2004.

[102] E. Fridman and U. Shaked, "On delay-dependent passivity," IEEE Transactions on Automatic Control, vol. 47, no. 4, pp. 664-669, April 2002.

[103] M. S. Mahmoud and L. Xie, "Stability and positive realness of time-delay systems," Journal of Mathematical Analysis and Applications, vol. 239, no. 1, pp. 7-19, 1999.

[104] Q. Li, Q. Zhang, N. Yi, and Y. Yuan, "Robust passive control for uncertain time-delay singular systems," IEEE Transactions on Circuits and SystemsPart I: Regular Papers, vol. 56, no. 3, pp. 653-663, March 2009.

[105] C.-W. Ho, A. E. Ruehli, and P. A. Brennan, "The modified nodal approach to network analysis," IEEE Transactions on Circuits and Systems, vol. CAS-22, no. 6, pp. 504-509, June 1975.

[106] R. W. Freund and F. Jarre, "An extension of the positive real lemma to descriptor systems," Optimization Methods and Software, vol. 19, no. 1, pp. 69-87, February 2004. 